Portland State University

PDXScholar

$1-27-1977$

\title{
Middle and upper Eocene biostratigraphy (Foraminifera) of the Cascade Head area, Lincoln and Tillamook Counties, Oregon
}

Arden D. Callender Jr.

Portland State University

Follow this and additional works at: https://pdxscholar.library.pdx.edu/open_access_etds

Part of the Geology Commons

Let us know how access to this document benefits you.

Recommended Citation

Callender, Arden D. Jr., "Middle and upper Eocene biostratigraphy (Foraminifera) of the Cascade Head area, Lincoln and Tillamook Counties, Oregon" (1977). Dissertations and Theses. Paper 2494.

https://doi.org/10.15760/etd.2491

This Thesis is brought to you for free and open access. It has been accepted for inclusion in Dissertations and Theses by an authorized administrator of PDXScholar. Please contact us if we can make this document more accessible: pdxscholar@pdx.edu. 
TO THE OFFICE OF GRADUATE STUDIES AND RESEARCH:

The members of the committee approve the thesis of Arden D. Callender, Jr. presented January 27, 1977.

Tichard E. Thoms, Chairman

John Eliot Allen

Josjeph $\sigma$. Kohlat

APP ROVED:

Marvin H. Beeson, Head, Department of Earth Sciences

Stanley E. Rauch, Dean of Graduate Studies and Research 
AN ABSTRACT OF THE THESIS OF Arden D. Callender, Jr. for the Master of Science in Geology presented January 27, 1977.

Title: Middle and Upper Eocene Biostratigraphy (Foraminifera) of the Cascade Head Area, Lincoln and Tillamook Counties, Oregon.

APPROVED BY MEMBERS OF THE THESIS COMMITTEE:

Ríchard E. Thoms, Chairman

John Eliot Allen

jos eph/J. / Kophut

An almost complete sequence of middle and upper marine strata, informally designated in this study as the "Strata of Cascade Head", has yielded 334 species and varieties of fossil Foraminifera. The Foraminifera were collected from 38 localities in four stratigraphic sections measured along the Salmon River, Neskowin Creek, Cascade Head Road, and near the town of Three Rocks in the central Oregon coast Range. Five species and one variety included within the genera Dentalina, Elphidium, Nodosaria (?), Nonion, Uvigerina, 
and Vulvulina are described as new but are not formally designated. Chilostomelloides eocenica and Triloculina tricarinata are reported for the first time from Eocene deposits on the West Coast.

Ecologically diagnostic Foraminifera indicate that the majority of this sequence was deposited at depths comparable to the upper continental slope, although at times marine deposition fluctuated between middle neritic and middle bathyal depths. While benthonic forms suggest cool bottom conditions, the diversity of planktonic species indicate warm surface temperatures and generally open connections with ocean currents.

The lower portion of the Neskowin Creek Section is assigned to the upper Ulatisian Stage (Amphimorphina californica zone) of Mallory whereas the balance of the "Strata of Cascade Head" can be correlated with Mallory's lower Narizian Stage (Bulimina corrugata zone). Also, the Uvigerina garzaensis subzone is recognized for the first time north of California in the Neskowin Creek Section. The "Strata of Cascade Head" correlates in part or as a whole in Oregon with the Coaledo? Formation of Baldwin, the Eocene beds at Cape Arago, the Moody Shale Member of the Toledo Formation, the Elkton Siltstone, Lorane Siltstone, and Sacchi Beach Members of the Tyee Formation, and the Yamhill Formation. In Washington it correlates with the Aldwell Formation, possibly the Crescent Formation, the Boundary Shale 
Member of the Lyre Formation, the "Maynard Formation" of Thoms and Allison, and the McIntosh Formation. Californian equivalents include the Butano Formation, the Canoas Siltstone Member of the Kreyenhagen Formation, the Cozy Dell Shale, the Domengine Sandstone, the Markley Sandstone, the Matilija Formation, the Muir Sandstone, the Nortonville Shale, the Poway Conglomerate, and the Vacaville Shale. 


\title{
MIDDLE AND UPPER EOCENE BIOSTRATIGRAPHY (FORAMINIFERA) \\ OF THE CASCADE HEAD AREA, \\ IINCOLN AND TILLAMOOK COUNTIES, OREGON
}

\section{by}

ARDEN D. CALLENDER, JR.

A thesis submitted in partial fulfillment of the requirements for the degree of

\author{
MASTER OF SCIENCE \\ in \\ GEOLOGY
}

Portland State University

1977 


\section{ACKNOWLEDGEMENTS}

I would like to express my sincere gratitude to $\mathrm{Dr}$. Richard E. Thoms, who, as my advisor in the graduate program at Portland State University, originally suggested the project and has given generously of his time and advice during all phases of this study. His professional guidance and encouragement has helped immensely in making this paper a reality. Other members of the faculty and staff at Portland State University who at one time or another have offered helpful advice or criticism include Dr. J. E. Allen, Dr. M. H. Beeson, Dr. P. E. Hammond, Dr. J. J. Kohut, Mr. D. E. Pierson, and Dr. R. O. Van Atta.

I am greatly indebted to $\mathrm{Mr}$. Wesley oleson and $\mathrm{Mr}$. Phillip Henry of Portland State University for their valuable assistance in the field during the summers of 1972-74; to Dr. G. R. Hornaday and Mr. F. J. Phillips of the University of California-Berkeley for allowing me access to the collections at that university; to Dr. Weldon $w$. Rau of the Washington State Division of Mines and Geology in Olympia for his aid and cooperation in discussing many of the problems of foraminiferal taxonomy; to Dr. P. D. Snavely, Jr. of the United States Geological Survey at Menlo Park, California for a helpful discussion of the stratigraphy of the Cascade Head area and for the many publications and maps 
he provided; to Textronix, Inc. of Portland, Oregon, especially Mr. Don Chitwood, for use of their scanning electron microscope; to Miss Deborah A. Edgerton of Portland State University who figured some of the Foraminifera for this study; to several dairymen in the Cascade Head area who allowed me access to their lands; and to $\mathrm{Mr}$. David $\mathrm{C}$. Lofgren of the Federal Highway Commission in Vancouver, Washington, Mr. Paul L. Smith, a graduate student at McMasters University in Hamilton, Ontario, and Mr. David G. Taylor, a graduate student at the University of CaliforniaBerkeley for their valuable criticism and helpful comments. My deepest thanks to all of these people.

Finally, I would like to give special thanks to my parents for their encouragement during this study and to my fiance, Christie Mayer, whose patience, support, and assistance in preparing the manuscript made this study possible. 
TABLE OF CONTENTS

PAGE

ACKNOWLEDGEMENTS . . . . . . . . . . . . . .

LIST OF FIGURES . . . . . . . . . . . . . . .

vii

INTRODUCTION . . . . . . . . . . . . . .

GEOLOGY . . . . . . . . . . . . . .

Introduction .............. . . 5

Siletz River Volcanic Series......... 8

"Strata of Cascade Head" ........... . 10

Neskowin Creek Section........ 17

Salmon River Section ......... 21

Three Rocks and Cascade Head Road Sections, 23

Volcanics of the Nestucca Formation . . . . . 24

PALEONTOLOGY . . . . . . . . . . . . . . 27

The Foraminifera . . . . . . . . . 27

Other Fossils . . . . . . . . . . . 28

BIOSTRATIGRAPHY ................... . . 45

Local Biostratigraphy . . . . . . . . 45

Paleoecology .............. . . 4 45

Amphistegina-Cibicides zonule ..... 47

Bulimina-Bolivina Faunule ....... 51

Bulimina-Nodosaria Zonule ....... 53

Chilostomelloides-Rhabdammina Faunule . . 55 
Nodosaria-Dentalina Zonule......

Bathysiphon-Spiroplectammina Faunule. .

Bolivina Zonule ...........

Uvigerina Zonule.......... 60

Age and Correlation . . . . . . . . . 62

Geologic History . . . . . . . . . . . 74

SYSTEMATIC CATALOGUE . . . . . . . . . . . 77

DESCRIPTIONS OF LOCALITIES . . . . . . . . . . 189

BIBLIOGRAPHY . . . . . . . . . . . . . . 197

PLATE •. . . . . . . . . . . . . . . . 211 


\section{IIST OF FIGURES}

FIGURE

PAGE

1. Index Map . . . . . . . . . . . . . . . 2

2. Geologic Strip Map, Neskowin Creek . . . . . . . 15

3. Geologic Strip Map, Salmon River . . . . . . . 16

4. Stratigraphic section: Neskowin Creek . . . in pocket

5. Stratigraphic section: Salmon River . . . . . . 22

6. "Strata of Cascade Head": Correlation of Sections 26

7. Composition of the Foraminiferal Fauna from the "strata of Cascade Head" . . . . . . in pocket

8. Distribution of Foraminifera from the "strata of Cascade Head". . . . . . . . . . . 31

9. Depth and Temperature Variation in the Neskowin Creek Section .. . . . . . . . . . . 48 


\section{INTRODUCTION}

The Coast Range of Oregon is composed of a complex of deeply dissected, steep sided ridges that roughly parallels the oregon Coast, and extends inland for 50 miles to the Puget-Willamette Trough and from the Columbia River southward some 250 miles to the Klamath Mountains. Deep weathering, landslides, and dense vegetation over much of the area obscure most of the underlying rocks and limit their exposure to stream beds and sea cliffs along the coast. In the vicinity of Cascade Head, on the western flanks of the central Oregon Coast Range, a structurally uncomplicated almost complete sequence of Eocene rocks is exposed along Neskowin Creek (Fig. 1-A). The sequence includes in ascending stratigraphic order, upper portions of the Siletz River Volcanic Series, "Strata of Cascade Head" of this report, and the volcanics of the Nestucca Formation.

Detailed geologic mapping of the intervals exposed for 3.5 miles along Neskowin Creek was accomplished during the summer and Fall of 1972-74. The major stratigraphic section (Fig. 1-A), nearly 3650 feet thick, was measured on a traverse along the creek by Brunton compass and tape, using the United States Geological Survey Map OM 97 (Snavely and Vokes, 1949) as a base. Because upper and 

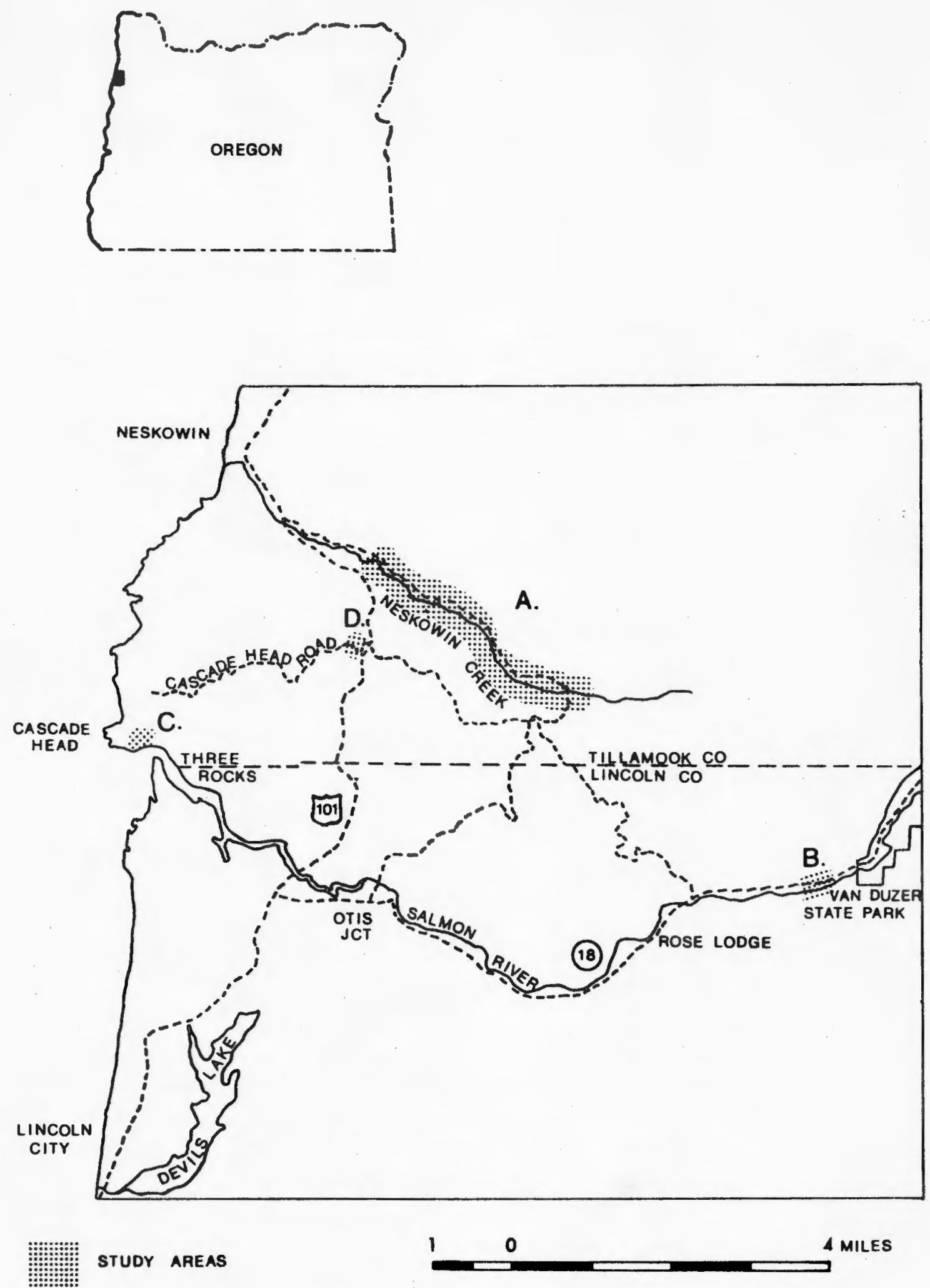

FIGURE 1. INDEX MAP 
lower contacts with the "Strata of Cascade Head" were not exposed in this section, three supplementary sections were measured in adjacent areas where the contacts are more clearly defined. On such section was measured along the Salmon River (Fig. 1-B) where the lower contact with the Siletz River Volcanic Series is exposed. Farther to the west, contacts with the overlying volcanics of the Nestucca Formation were observed and sections were measured near the town of Three Rocks and on Cascade Head Road (Fig, 1-C,D). Initially, 124 samples were collected from the Cascade Head area for this study. Finer-grained samples were disaggregated by the kerosene-water technique. More indurated samples, cemented by calcite, were broken down in a soil disaggregator. One hundred fifty grams of each sample was disaggregated, washed, and inspected for fossil content. Thirty percent of the samples (38 of the 124 samples) contained well preserved foraminiferal assemblages suitable for a biostratigraphic study.

These foraminiferal assemblages were evaluated in an attempt to gain further information pertaining to the geologic age, environment of deposition, and correlation of rocks designated in this investigation as the "Strata of Cascade Head". In addition, an effort was made to refine the local stratigraphic and structural problems in the area, and to a lesser degree, to investigate the physical relationships between these rocks and others 
exposed within the Cascade Head area.

Regionally, the relatively uncomplicated sequence exposed in the Cascade Head area is important for its occurrence along the southern margin of a thick, complex sequence of Eocene volcanics named the Tillamook Volcanic Series. Knowledge of the biostratigraphy of the "Strata of Cascade Head", which is in part equivalent to the Tillamook Volcanic Series, should therefore aid in understanding the more complex stratigraphy of this vast accumulation of Eocene volcanics in northwestern Oregon. 


\section{GEOLOGY}

\section{Introduction}

A sequence of more than 3630 feet of marine sedimentary and volcanic rocks comprising the "Strata of Cascade Head" and portions of the underlying Siletz River Volcanic Series and overlying volcanics of the Nestucca Formation is exposed in the vicinity of Cascade Head on the western flanks of the central Oregon Coast Range. The Siletz River Volcanic Series form rugged highlands and steep slopes in the eastern part of the area while gentler slopes are developed on the deeply weathered sediments of the "Strata of Cascade Head". Farther to the west, the volcanics of the Nestucca Formation form the prominent headland of Cascade Head. Exposure of these units within the Cascade Head area is almost entirely restricted to the two major westward flowing streams (Salmon River and Neskowin Creek) that traverse the area.

The Coast Range Province is composed of a series of gently folded Tertiary marine sedimentary and volcanic rocks which, east of the Cascade Head area, has been uplifted into a broad anticlinorium. In the central oregon Coast Range, the Siletz River Volcanic Series and the Tyee Formation to the south form the central core of this structural high. Unconformably overlying these early 
Tertiary sequences is a series of predominately marine sedimentary and volcanic rocks which is exposed on the flanks of the upwarp. On the eastern flank, the Tyee Formation grades laterally to the north into sediments of the Yamhill Formation. Numerous west-dipping sequences, ranging in age from middle Eocene to Miocene, are exposed on the western flank and form a narrow band that parallels the oregon Coast. Basaltic dikes and sills of probable Miocene age (Snavely and Vokes, 1949) occur abundantly throughout the area.

The areal extent of the "Strata of Cascade Head" appears to be quite limited. Its occurrence is essentially restricted to an area between Neskowin Creek and the Salmon River in Sec. 29 and 30, T6S, R9W. Strata of equivalent age, but different lithology, are exposed to the north along the Nestucca River and to the south near Devils Lake. Snavely, MacLeod, and Wagner (1972) include these sequences within the "Yamhill Formation". East of the Cascade Head area, more typical sediments of the Yamhill Formation crop out along the eastern margins of the central oregon coast Range. Presumably the "Strata of Cascade Head" are either interbedded with or grade laterally into all of the aforementioned units. Further detailed geologic mapping may help resolve the nature of these contacts and the true areal extent of the "Strata of Cascade Head". The thickest, most complete section of the "Strata of Cascade Head" is exposed along Neskowin Creek. Although 
the subjacent and superjacent units are exposed in the section, the contacts with them are covered, and consequently are best described in areas to the east and west of the creek (Fig. 1-B,C,D). The "Strata of Cascade Head" rest with apparent unconformity upon volcanic breccias of the Siletz River Volcanic Series in both the Neskowin Creek and Salmon River Sections. This interpretation is strengthened by the presence of a basal sandstone unit within the "strata of Cascade Head" in the Neskowin Creek Section, that directly overlies the volcanic breccias and contains numerous basaltic fragments of a composition similar to that of the underlying siletz River Volcanic Series. At the mouth of the Salmon River near the town of Three Rocks, basaltic breccias of the volcanics of the Nestucca Formation unconformably overlie the "Strata of Cascade Head". Snavely (personal communication, 1976) reports a regionally recognizable unconformity, representing a major period of mid-to-late Eocene deformation and erosion, at the base of the Nestucca Formation. Evidence for this mid-to-late Eocene unconformity within the local area cannot be substantiated. Although the samples collected below the contact contained abundant Foraminifera, samples collected from sedimentary interbeds within the volcanics lacked diagnostic Foraminifera.

The dominant structural feature within the Cascade Head area consists of a northeast plunging anticline whose 
axis lies just east of the town of Dolph (Snavely and Vokes, 1949). The regional strike parallels this fold axis and strata exhibit gentle dips on either side of the fold; dips average less than $20^{\circ}$ to the southeast and northwest. Secondary folding on the western flank is evidenced in the increased outcrop thicknesses as noted by Snavely and Vokes (1949), and in a series of minor folds mapped along Neskowin Creek by the present author. Only minor faulting affects the major section along Neskowin Creek. The continuity of foraminiferal samples across numerous covered intervals lends support to this interpretation.

In the following discussion, all thicknesses refer to stratigraphic thickness unless otherwise noted. Rocks were identified in hand specimen except in a few cases in which thin sections were examined. Lithologic descriptions are depicted graphically in Figures 4 and 5 (Fig. 4 in pocket). For further detailed descriptions of the petrology of the rocks in the central oregon coast Range the reader is referred to the works of Snavely and Baldwin (1948), Snavely and Vokes (1949), Snavely and Wagner (1961), (1964), and Snavely, MacLeod, and Rau (1969a).

\section{Siletz River Volcanic Series}

The Siletz River Volcanic Series was defined and described by Snavely and Baldwin (1948) as a sequence of basalt flows, pillows, flow breccias, pyroclastics, and minor amounts of water-laid tuff exposed along the Siletz 
River in the Euchre Mountain and Valsetz Quadrangles and adjacent areas. The base of the sequence is not exposed, but estimates of thickness range from approximately 500 feet in the southwest corner of the Sheridan Quadrangle to more than 10,000 feet in the Mary's Peak area (Baldwin, et al., 1955). This unit, in conjunction with portions of the Tillamook Volcanic series to the north and volcanics of the Umpqua Formation to the south, represents the oldest rocks exposed in the Oregon Coast Range and the major result of early Tertiary submarine volcanism in oregon.

In the Cascade Head area, the siletz River Volcanic Series forms a distinctive base for sections measured along Neskowin Creek and the Salmon River. Approximately 60 feet of olive-blackl, basaltic breccia, referable to this unit, is exposed in a number of small isolated outcrops along Neskowin Creek east of the bridge over the creek, three-fifths of a mile east of Neskowin Creek Campground. The outcrops characteristically lack bedding and commonly are cut by many thin, discontinuous calcite and zeolite veinlets. Exposures are deeply weathered and support a luxuriant growth of mosses and ferns. The first outcrop of the "Strata of Cascade Head" is exposed 29 feet west of the breccia. The southern portion of an extensive basaltic sill is exposed along the creek east of the breccia after

$l_{\text {Color terms in this paper follow the Geological }}$ Society of America Rock-color chart (Goddard, 1970). 
approximately half a mile covered interval.

In contrast to the Neskowin Creek Section, approximately 5000 to 6000 feet of rocks referable to the Siletz River Volcanic Series (Snavely and Vokes, 1949) are exposed along the Salmon River which traverses the

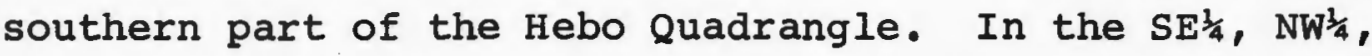
Sec. $30, T 6 S, R 9 W$, less than 10 feet of basaltic breccia unconformably underlie the "Strata of Cascade Head". The breccia is well-bedded, olive-black in color and contains smaller basaltic fragments than those observed in the breccias exposed along Neskowin Creek. To the west it grades rapidly into numerous basalt flows which characterize the rest of the sequence exposed along the Salmon River.

A few samples collected from the finer-grained breccias were examined for foraminiferal content, but were found to be completely devoid of microfossils. However, foraminiferal assemblages as well as megafossils have been reported from sedimentary interbeds within the Siletz River Volcanic Series to the east and south of the Cascade Head area (e.g. Snavely, Macleod, and Rau, 1969a). Faunae from the sedimentary interbeds indicate that the volcanics are referable to Mallory's (1959) Ulatisian and possibly Penutian Stages.

\section{"Strata of Cascade Head"}

The first comprehensive geological report of the coastal area between Cape Kiwanda and Cape Foulweather was 
published by Snavely and Vokes in 1949. In the report, they proposed the name Nestucca Formation to include a sequence of "brackish water and marine siltstone and claystone, sandstone, and intercalated volcanic material" which overlies the Siletz River Volcanic Series and Burpee (Tyee) Formation. A complete section is not exposed but estimates of thickness were approximately 7000 to 8000 feet in the Hebo Quadrangle. Two type sections were designated as occurring "along the north bank of the Salmon River from Otis to Three Rocks and in roadcuts along the north side of Nestucca Bay."

Initially, the present investigation was undertaken to evaluate foraminiferal assemblages from the type Nestucca Formation. Unfortunately the two type sections designated by Snavely and Vokes (1949) were not suitable for a biostratigraphic study; the section along the salmon River is frequently obscured by landslides and the outcrops to the north on Nestucca Bay are noncontinuous, deeply weathered, and generally lack Foraminifera. However, between the two sections, a thick sequence of marine sedimentary and volcanic rocks, originally referable to the Nestucca Formation, is exposed along Neskowin Creek. The main emphasis of this study was placed upon these sedimentary rocks that comprise the lower member of the Formation.1

${ }^{1}$ Prior to Snavely's redesignation, the Nestucca Formation within the Cascade Head area was subdivided into three informal units by this author; a sedimentary lower member, a volcanic middle member, and a sedimentary upper member. 
Subsequently, Snavely (personal communication, 1976) has restricted the use of the term Nestucca Formation to include only the middle and upper members exposed to the northwest of the measured sections. The lower member is assigned to the "Yamhill Formation". Thus, strata previously mapped as the lower Nestucca Formation (Snavely and Vokes, 1949) along the coast between Cape Kiwanda and Cape Foulweather are now referable to the "Yamhill Formation" of Snavely, MacLeod, and wagner (1972). It should be noted however, that rocks assigned to the "Yamhill Formation" of Snavely, et al. (1972) which occur immediately to the north and south of the Cascade Head area, differ somewhat from the sediments of the type Yamhill Formation exposed some 25 miles to the east along Mill Creek. Strata of the type Yamhill Formation are predominately massive to thick-bedded and weather to a reddish-brown color whereas the outcrops along the Nestucca River and near Devils Lake are typically thin-bedded, more tuffaceous, and weather yellow-orange. These differences were observed during a reconnaissance of the areas surrounding Cascade Head and not as a result of detailed geologic mapping. The regional relationships between these units is unknown and beyond the scope of the present investigation.

The sequence exposed in the vicinity of Cascade Head however, differs conspicuously from both the sediments of 
the type Yamhill Formation and strata referable to the "Yamhill Formation" of Snavely, et al. (1972) exposed to the north and south of the Cascade Head area. These rocks are decidedly more coarse-grained and have been strongly influenced by probable contemporaneous volcanism. In the type section along Mill Creek, the Yamhill Formation is composed of primarily siltstone and mudstone with lesser amounts of basaltic sandstone exposed in the middle and at the base of the section (Gaston, 1974, p. 12-14). Outcrops exposed to the north and south of the Cascade Head area consist primarily of thin-bedded, tuffaceous siltstone and mudstone. In contrast, most of the sequence exposed along Neskowin Creek is characterized by coarse to mediumgrained sandstones that occur repeatedly throughout the section. Low in the sequence the sandstones are calcareous and often contain basaltic fragments. Above this is a small interval of micaceous, arkosic sandstone that rapidly grades upward into a thick sequence of volcanic wackes and graywackes which constitute the bulk of the section. E'vidence of contemporaneous volcanism is reflected in the basaltic debris and tuffaceous composition of the volcanic wackes and graywackes. Flows of basaltic lava, volcanic breccia, basaltic tuff, and occasional ash beds are also interbedded with the clastics. Finer-grained sediments consisting of well-indurated, tuffaceous siltstones and mudstones, although frequently consisting of thick 
sequences near the base of the section, are commonly interbedded with sandstones throughout most of the interval. Towards the top of the sequence rhythmically-bedded mudstone and volcanic sandstone become increasingly persistent. Most of the sediments exposed along Neskowin Creek, as well as the strata exposed to the north and south of the area, are characteristically thin-bedded and differ from the more massive to thick-bedded appearance of the type Yamhill Formation.

As a result of these differences, the rocks exposed in the vicinity of Cascade Head (Fig. 2,3) which occur above the Siletz River Volcanic Series and below the volcanics of the Nestucca Formation have been designated the "Strata of Cascade Head" in this investigation. The "Strata of Cascade Head" represent a coarse-grained, somewhat volcaniclastic facies of the finer-grained siltstones and mudstones of the "Yamhill Formation" of Snavely, et $a$. (1972). Although the lateral relationships of this unit are unknown, it seems probable that these rocks become interbedded with or grade laterally into strata of the "Yamhill Formation" of Snavely, et al. (1972) exposed to the north and south of the Cascade Head area and the Yamhill Formation to the east. If the term "Yamhill Formation" continues to be used in reference to the strata exposed in the areas surrounding Cascade Head, it is suggested that the rocks referred to informally as the 


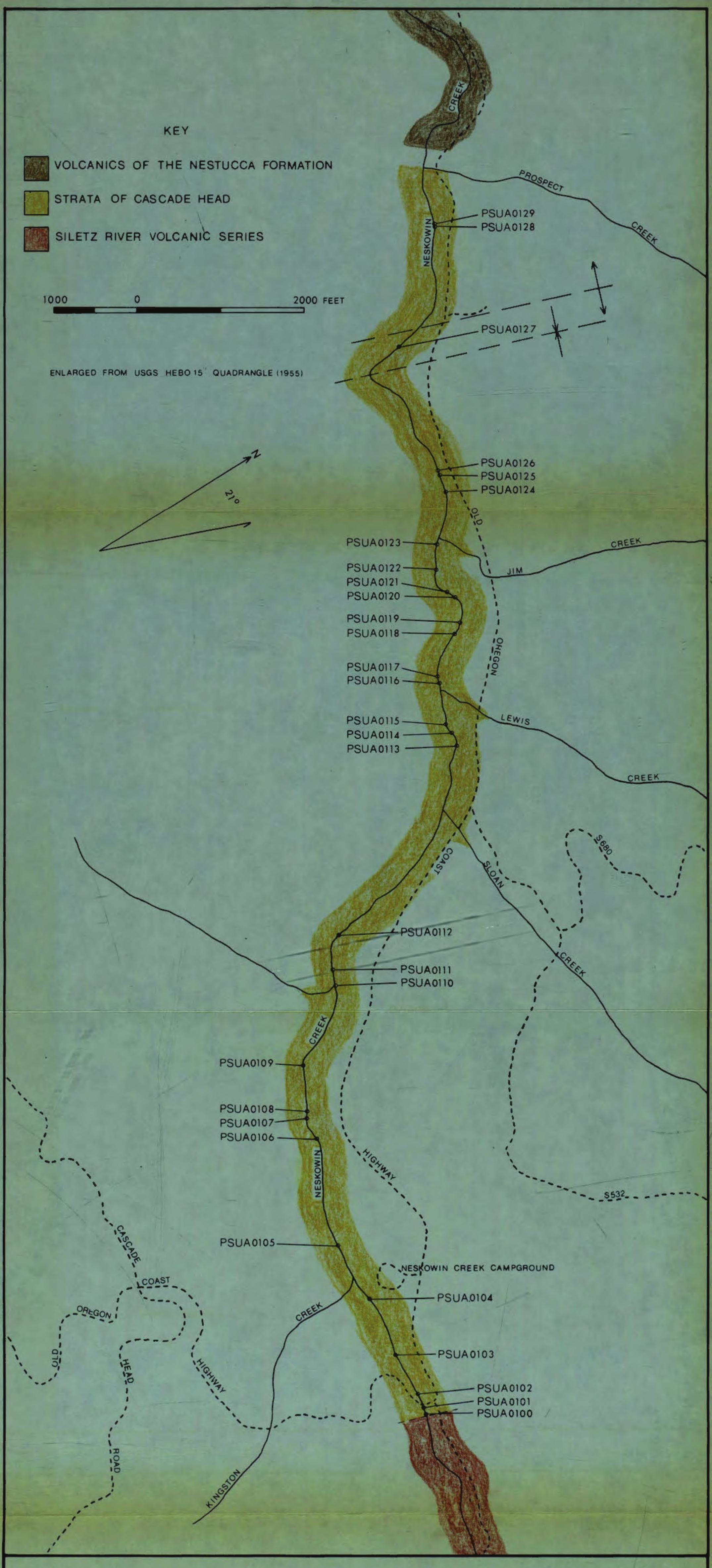

FIGURE 2. GEOLOGIC STRIP MAP, NESKOWIN CREEK 
"Strata of Cascade Head" be elevated to the status of a member (the Cascade Head Member of the Yamhill Formation). The type section is designated to include the rocks exposed along Neskowin Creek from the bridge over the creek in Sec. 15, T6S, R10W, downstream to a point just west of where Prospect Creek joins Neskowin Creek.

The following discussion refers to the stratigraphic sections measured during the present investigation.

\section{Neskowin Creek Section}

The "Strata of Cascade Head" are exposed in a series of westward dipping benches that traverse Neskowin Creek for 3.5 miles (Fig. 1-A). In the section measured along the creek, rocks referable to this unit attain a thickness of 3495 feet. 1 Numerous covered intervals that are generally associated with the thinly-bedded mudstone and siltstone units occur commonly throughout the sequence. Towards the top of the section, the sequence is partially repeated on the limbs of an anticlinal and synclinal fold. A basalt flow, exposed on the limbs, provides a convenient marker for lithologic correlation across the folds. The thinning of this basalt to the southeast indicates that the flow originated in an area somewhere to the northwest.

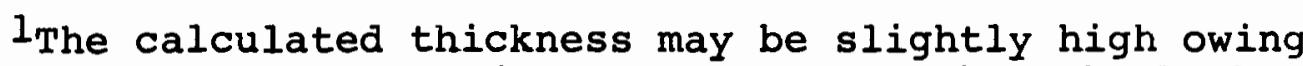
to the covered intervals (a total of 61 feet) at both the base and top of the section. The volcanic subjacent and superjacent units however, where observed in other areas, are very resistant to weathering and therefore the thickness given above seems reasonable. 
Intrusives, which occur commonly in the surrounding areas, were encountered only near the middle of the section where three olivine augite basalt dikes cut a sequence of micaceous arkosic sandstone.

The section measured along Neskowin Creek can be separated into three generalized subdivisions based upon its coarser-grained constituents: a lower calcareous volcanic sandstone interval (570 feet), a middle micaceous arkosic sandstone interval (948 feet), and an upper volcanic sandstone interval (1977 feet). The lower 570 feet of the section is characterized by medium to light-gray, mediumgrained, calcareous volcanic wacke. At the base of the section these well-indurated sandstones predominate in beds that seldom exceed 12 inches in thickness. Toward the top of the interval the calcareous sandstones become massive to faintly-bedded. Weathered surfaces have a pitted appearance because of clastic grains which have been etched in relief. Interbedded with the sandstones are minor amounts of thinly-bedded, olive-gray tuffaceous siltstone and mudstone that increase in thickness toward the center of the section and in places contain light-gray calcareous nodular concretions. In the middle part of the interval, the calcareous sandstones give rise to light-gray, finegrained arkosic wackes that contain occasional carbonaceous zones. These sandstones lack the finely disseminated micaceous material that distinguishes the overlying arkosic 
sandstone interval. Intercalated with the sandstone near the top of the sequence is a grayish-black, thickly-bedded, basaltic vitric tuff that contains quartz and calcite in veinlets and interstices. A light olive-gray lithic ash, altered to montmorillonite, is associated with this tuff. Although the 948 foot interval overlying the lower calcareous sandstone is composed primarily of olive-gray mudstone, this sequence is distinguished lithologically by thickly-laminated beds of light to medium-gray, finegrained, micaceous arkosic wacke. These sandstones become more prevalent toward the top of the interval where they are occasionally interbedded with or grade into micaceous siltstone. In fresh exposures, the sandstone is wellindurated, but it commonly weathers to a yellowish-orange, friable sand. The mudstones which form thick sequences in the lower two-thirds of the interval, are tuffaceous, thinly-bedded, and become progressively siltier toward the top of their exposure. Locally, light-gray calcareous concretions occur within both sandstone and mudstone units. Toward the top of the interval, flows of medium-gray basalt and olive-black basaltic breccia are intercalated with micaceous arkosic sandstones and silty mudstones. Calcite occurs quite frequently in veinlets in the breccia and basalt.

The uppermost interval, which directly overlies the micaceous arkosic sandstones, reaches a thickness of 1977 
feet in the Neskowin Creek Section. Strata typically consist of alternating thin beds of olive-gray to black, fine to coarse-grained volcanic wacke or graywacke and olive-gray micaceous mudstone or siltstone. These alternations rarely exceed six inches in thickness and the basal contacts of the sandstone beds with the underlying mudstone and siltstone units are frequently undulatory because of load deformation. Thicker units of mudstone, siltstone or sandstone occur sporadically throughout the interval. The volcanic sandstones, which differentiate this sequence from underlying intervals, locally contain subangular to subrounded basaltic pebbles apparently derived from contemporaneous volcanism. In places the sandstones are micaceous, calcareous or glauconitic and usually weather to a brownish or reddish-orange color. The mudstones and siltstones typically weather to a yellowishorange color and often contain tuffaceous and carbonaceous material. Light-gray calcareous concretions and nodules that parallel the bedding occur throughout the sequence. Near the top of the interval, a flow of medium-black basalt and vesicular basalt are intercalated with siltstone and sandstone units.

Numerous foraminiferal samples were collected principally from mudstone and siltstone units exposed along Neskowin Creek. Well preserved Foraminifera were found in 30 samples that are listed in the checklist under localities 
PSUA0100 through PSUA0129. Further information regarding lithology and stratigraphic relationships of these samples is given in Figure 4 (in pocket).

\section{Salmon River Section}

In order to gain additional stratigraphic control at the base of the "Strata of Cascade Head", a small 197 foot thick supplementary section was measured along the salmon River (Fig. 1-B). This sequence had previously been included within the Tyee Formation (Snavely and Vokes, 1949; Snavely, et al., 1972) but in this investigation it is assigned to the "Strata of Cascade Head". Lithologically, the rocks exposed in the Salmon River section differ from the rhythmically-bedded sandstone and mudstone of the Tyee Formation in that siltstone comprises most of the section. The fauna from the sequence also substantiates this interpretation; the Tyee Formation is assigned a late middle Eocene age (Beaulieu, 1971) whereas Foraminifera from the Salmon River section are indicative of an early late Eocene age. In addition, it should be noted that although the sediments exposed along the Salmon River have been assigned to the "Strata of Cascade Head", differences occur between these rocks and strata exposed less than four miles away in the Neskowin Creek section. In the Salmon River Section, micaceous arkosic wackes interbedded with tuffaceous siltstones occur at the same stratigraphic interval occupied by the lower calcareous sandstones in the 


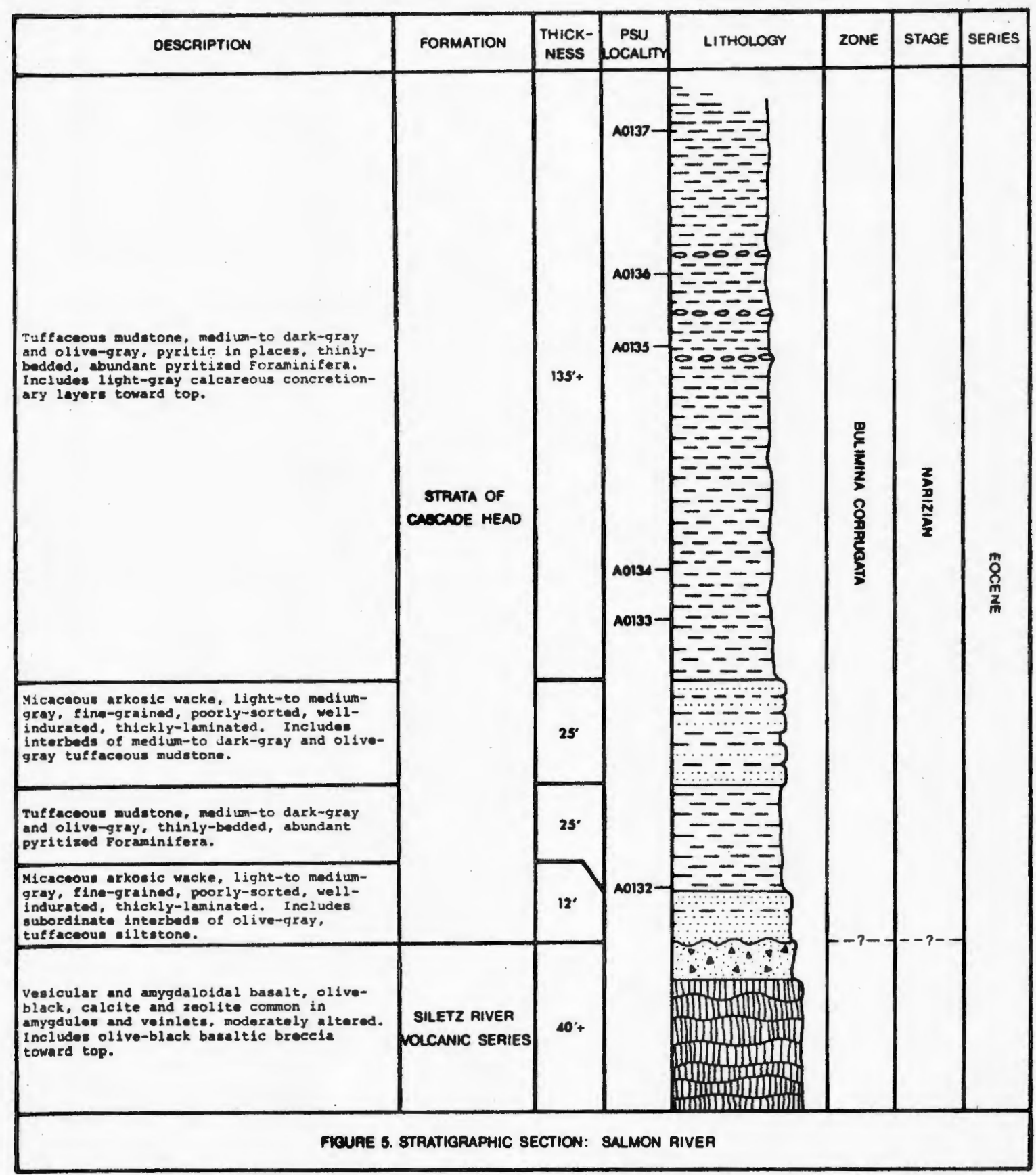


Neskowin Creek section. Since only the base of the sequence was examined in this investigation, further detailed geologic mapping along the Salmon River will be needed to demonstrate that this section belongs within the "strata of Cascade Head". The absence of a lower calcareous sandstone interval in the Salmon River section is discussed further on p. 75 .

The majority of the section measured along the salmon River is composed of medium to dark-gray and olive-gray, thinly-bedded siltstone and mudstone. Near the base of the sequence thickly-laminated beds of light to medium-gray, fine-grained micaceous arkosic wacke are interbedded with the siltstone and mudstone. These well-indurated sandstones appear quite similar to the micaceous arkosic sandstones exposed in the Neskowin Creek Section. Light-gray calcareous concretionary layers and nodules are associated with the siltstone and mudstone units near the middle of the sequence. Toward the top of the section, the sequence is cut by a olive-black basaltic dike.

Foraminifera, usually consisting of pyritized internal molds and casts, were identified from six samples collected from the finer-grained sediments and are listed in the checklist under localities PSUA0132 through PSUA0137.

\section{Three Rocks and Cascade Head Road Sections}

Because of limited exposure at the top of the Neskowin Creek Section, supplementary sections were sampled 
at the mouth of the Salmon River near the town of Three Rocks and along Cascade Head Road approximately half a mile from its junction with Highway 101 (Fig. 1-C,D). In both sections the rocks are composed of thinly-bedded, olivegray tuffaceous mudstone with minor amounts of light olivegray tuffaceous siltstone. The Cascade Head Road section is cut by numerous medium-black basaltic dikes. Well preserved Foraminifera occur abundantly throughout both sequences and were identified from two localities that are listed in the checklist under localities PSUA0130 (Cascade Head Road) and PSUA0131 (Three Rocks).

Volcanics of the Nestucca Formation

Although the Nestucca Formation is composed primarily of tuffaceous siltstone and sandstone and in places arkosic sandstone, more than 1000 feet of basalt flows, basaltic breccia, pillows, lapilli tuff, and minor amounts of interbedded siltstone is exposed at the base of the formation in the vicinity of Cascade Head. This sequence thins to the northeast, where it interfingers with sedimentary rocks of the Nestucca Formation (Snavely and Vokes, 1949). The "Strata of Cascade Head" are overlain by this sequence of volcanic rocks in the Cascade Head area and presumably by the sedimentary rocks to the northeast. The volcanics of the Nestucca Formation form a lithologically distinct top to the three sections measured along Neskowin Creek, near Three Rocks, and along Cascade Head Road. In 
all three sections the rocks directly overlying the "strata of Cascade Head" are composed of olive-black basaltic breccia often containing calcite and zeolite in veinlets. Three foraminiferal samples were collected from siltstone interbeds within the volcanics of the Nestucca Formation during a reconnaissance along Neskowin Creek. Two of the samples were completely devoid of microfossils and the third contained only one non-diagnostic species, Bathysiphon eocenicus. However, well preserved Foraminifera have been reported from the sedimentary rocks of the Nestucca Formation exposed to the north and south of the Cascade Head area that indicate these rocks are of latest Eocene age (Snavely and Vokes, 1949). 


\section{PALEONTOLOGY}

\section{The Foraminifera}

The sequence of rocks exposed in the vicinity of Cascade Head, although possessing few larger fossils, contains an abundant and diverse microfauna. A total of 334 species and varieties of Foraminifera, referable to 81 genera and 27 families, were obtained from 38 localities. The calcareous perforate forms are decidedly dominant throughout the interval and account for 89 percent of the total assemblage. The arenaceous and calcareous imperforate groups, although important in some assemblages, occur less frequently and account for 11 percent of the total fauna (Fig. 7 , in pocket).

The arenaceous Foraminifera are represented by 18 genera from 10 families, and include 40 species. They occur in subordinate amounts in most assemblages and are dominated by such genera as Rhabdammina, Bathysiphon, Haplophragmoides, Spiroplectammina, Karreriella, and Tritaxilina. The calcareous imperforate Foraminifera, particularly common in the basal Neskowin Creek Section, occur more sporadically throughout the section and include 11 species assigned to five genera from two families. The calcareous perforate Foraminifera are represented by 283 species referable to 58 genera from 15 families and, 
as previously mentioned, are the dominant group in the sequence. The families represented by genera possessing the greatest number of individuals are as follows: Lagenidae by Robulus, Dentalina and Nodosaria; Nonionidae by Nonion; Heterohelicidae by PZectofrondicularia and Nodogenerina; Buliminidae by Bulimina, Bolivina, and Uvigerina; Ellipsoidinidae by Ellipsonodosaria; Rotaliidae by Gyroidina, Eponides, and Cancris; Amphisteginidae by Amphistegina; Cassidulinidae by Cassidulina; Chilostomellidae by ChizostomeZloides; Globigerinidae by GLobigerina and Subbotina; and Anomalinidae by Cibicides.

An analysis of the faunas, by the percentage of each family as compared with the total faunal assemblage from the Cascade Head area, is shown in Figure 7. A complete list of the Foraminifera including their relative abundance in each locality is given in Figure 8.

\section{Other Fossils}

Numerous other microfossils were encountered during the course of the present study. Specific identification of these forms was not attempted since the emphasis of this report is placed primarily on the benthonic Foraminifera. The specimens however, were retained, mounted in the appropriate assemblage slide, and their occurrence and relative abundance noted in Figure 8 . This group includes coral, diatoms, fragments of Dentalium, echinoderm plates and spines, fecal pellets, fish bone, teeth, and scales, 
immature gastropods, other mollusk fragments, ostracods, placoid scales, Radiolaria, scolecodonts, shark teeth, and sponge spicules. Several of these forms deserve special notice.

Strata from the Cascade Head area are characterized by numerous Radiolaria which occur consistently throughout the sequence. In some localities, they make up over 50 percent of the assemblages and in samples devoid of Foraminifera, they may constitute as much as 100 percent of the total fauna. Although a great number of individuals were observed, very few species are represented. Diatoms, echinoderm spines, ostracods and sponge spicules also occur in significant numbers throughout the sequence. Their relative abundance and stratigraphic range in shown in Figure 8.

A single fragment resembling the jaw of a polychaeta worm or scolecodont was one of the more interesting elements of the microfauna collected from the Neskowin Creek Section (PSUA0124). The slender, reddish-brown fragment is capped by three conical "tooth-like" projections and may represent a portion of the pharyngeal jaws known as the forceps. To the author's knowledge, scolecodonts have not previously been recorded from the Tertiary deposits of Oregon.

Larger marine invertebrates, in contrast to the abundant microfauna, occur very infrequently in strata exposed in the Cascade Head area. A molluscan fauna was 
described by H. E. Vokes (Snavely and Vokes, 1949) from a locality (MF-23) on the Salmon River near the town of Three Rocks. The locality, which is located approximately 500 feet southeast to PSUA0131 of this study, represents the only reported occurrence of macrofossils within the area of investigation. Vokes identified the following mollusks:

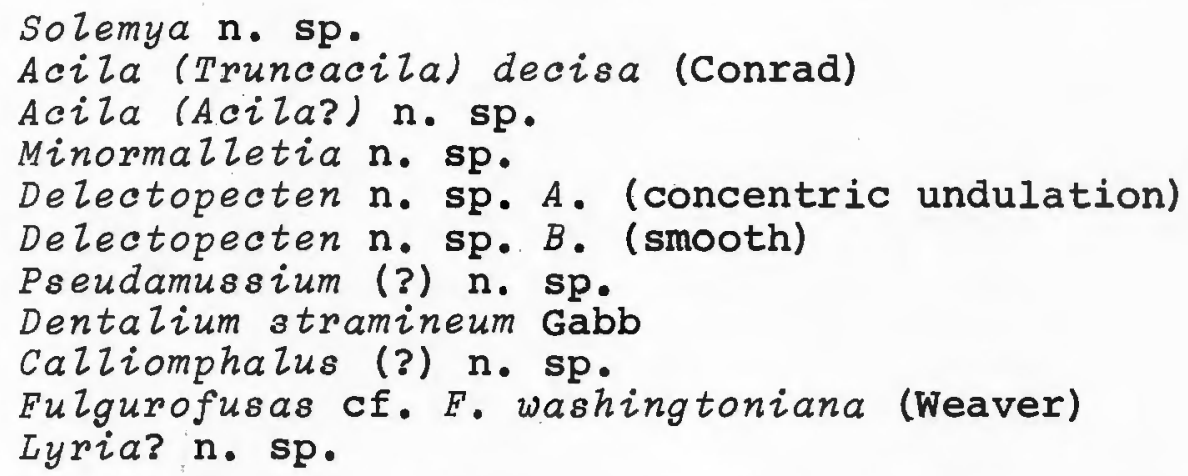

Vokes concluded that the fauna was of late Eocene age and correlated it with the faunas of the Moody Shale Member of the Toledo Formation, and with the Cowlitz and Coaledo Formations. A few poorly preserved mollusks from the Neskowin Creek section were the only megafossils observed during this investigation. They were collected from a pebbly volcanic graywacke located 50 feet stratigraphically above PSUA0121. The fauna includes Acila sp., Ostrea sp., Dentalium sp., and fragmented low-spired gastropod. 


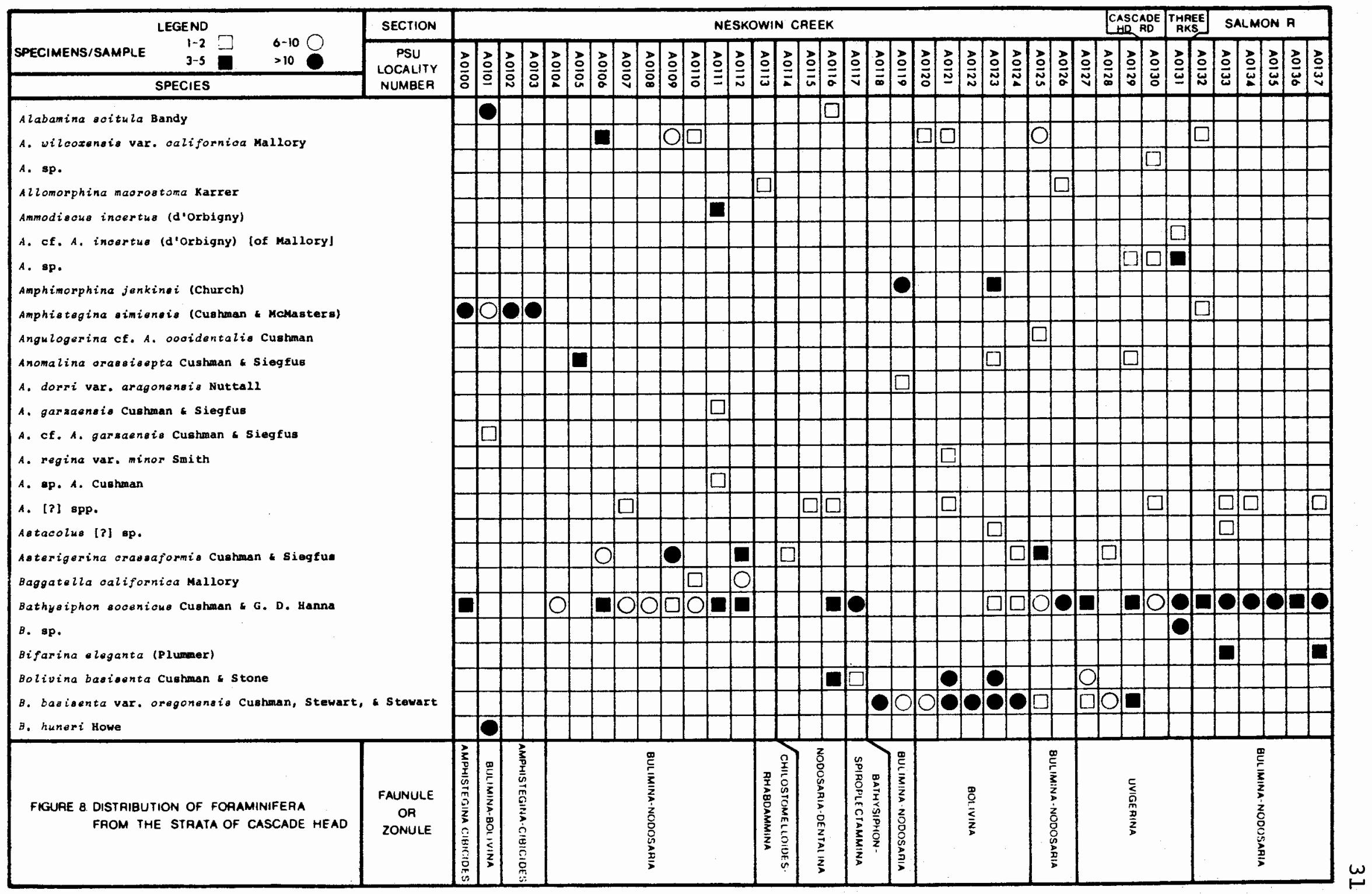




\begin{tabular}{|c|c|c|c|c|c|c|c|c|c|c|c|c|c|c|c|c|c|c|c|c|c|c|c|c|c|c|c|c|c|c|}
\hline LEGEND & SECTION & & & & & & & & & & & Skow & IN $\mathrm{C}$ & AEEK & & & & & & & & & & & $\begin{array}{l}C A D E \\
\text { CAD }\end{array}$ & THRE & & SALM & ION R & \\
\hline $\begin{array}{lll}\text { SPECIMENS/SAMPLE } & 1-2-5-5 & 0.10 \\
3-5 & >10\end{array}$ & $\begin{array}{c}\text { PSU } \\
\text { LOCALITY }\end{array}$ & : & & & $\overrightarrow{0}$ & & & ? & : & $\vec{a}$ & & & 0 & 후음 & 음 & & & : & & & o & 它 & : & & 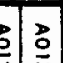 & & & & & 히음 \\
\hline SPECIES & & & & & & & & & & & & $\bar{\omega} \mid \bar{\omega}$ & & & & क्ष & 5 & & & $\tilde{\omega}$ & & & & & & 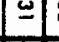 & & \begin{tabular}{c|c|c|} 
\\
\end{tabular} & & 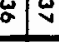 \\
\hline Bolivina jaaksonenais Cushran a Applin & & & & & & & {[} & & & & & & & & $\square$ & & & & & & $\square[$ & $\square$ & & & & & & & & \\
\hline B. Kleinpelli Beck & & & O & & & & & & & & & & & & & & & & & & & & & & & & [ & a & & 0 \\
\hline B. Soabrata [3] Cushman a Bermudez & & & & & & & & & & & & & & $\square$ & & & & & & & L & 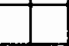 & & & & & & & & \\
\hline B. spp. & & & & & & & $\square$ & & & & & & & $\square$ & O & & 更 & O. & D. & O & & (i) & & 0 & & & & & & a \\
\hline Butimina coneanguinea Parkex \& Bermudez & & & & & & 마 & & & & & & & & & & & & & & & & $\Rightarrow$ & & & & & & & & \\
\hline B. corrugata Cushman a siegfus & & & Q & & 0 & & O & 0 & 0 & O & $\square$ & & & E & & & $\square$ & & e & 0 & & $\square$ & & & $\square$ & & of & $\theta$ & & 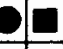 \\
\hline 8. curtiasima Cumhman a Siegfus & & & & & & & OE & $\square$ & a & & $\square$ & & & & & & & & & & & $\square$ & & & & & $\square$ & & & \\
\hline B. cf. B. ourtiseime Cushman a siegfus & & & & & & & & $\square$ & & & & & & & & & & & & & & & & & & & & & & \\
\hline B. decurtata Cushman \& Stainforth & & & & & & & & $\square$ & & & $\square$ & & & & & Q & $\mathrm{O}$ & & & 0 & & $\square$ & & & & & & & & \\
\hline B. Elongata d'orbigny & & & & & & & & & & & & & & & & & & & & $\square$ & & & & & & & & & & \\
\hline B. gwayabalensis var. ampla [?] Cushman \& Parke & & & & & & & $\square$ & & & & & & & & & & & & & & & $\square$ & & & & & & & & \\
\hline B. instabilis [?] Cunhman a Parker & & & & & & & & & & & & ] & & & & & & & & & & & & & & & & & & \\
\hline B. jarvisi Cushman \& Parker & & & & & & & & & & & & & & & D & & & & & & & & & & & & & 0 & & \\
\hline B. Lirata Cuahman \& Parkex & & & & & & & & & & & & & & & & & 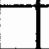 & & & & & & & & & & & $\square$ & & \\
\hline B. miorooostata Cushman G Parker & & & & & & & 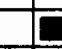 & 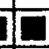 & & & & & & & & & O & & 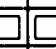 & & 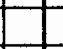 & & & & & & & & & \\
\hline B. ovata d'orbigny & & & & & $\square$ & & & & & & $\square$ & & & & & & & & & & & $\square$ & & & & a & & 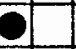 & $\square$ & \\
\hline B. ovata [?] d'orbigny & & & & & & & & & & $\square$ & & a & & & 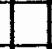 & & & & & & & $\square$ & & & & $\square$ & & & & \\
\hline B. paeheooensis Smith & & & O & & & & & & $\bar{O}$ & & & $\square$ & & & & & & & & & & & & & & & & 9 & & \\
\hline B. paoheooeneio [2] Smith & & & 1 & & & & & & & & & & & & & & & & & & $\mathrm{Q}$ & & & & & & $\square$ & & & \\
\hline B. pupoides d'orbigny & & & & & & & & & & & $\square$ & & & & & $\square$ & & & & & & $\mathrm{O}$ & & & & & $\square$ & 0 & & OL \\
\hline B. Cf, B. reusei morrow [of weaver] & & & O & & & & & & & & & & & & & & $\square$ & & & & & & & & & & & 0 & & \\
\hline B. Bchenaki Beck & & & & & & & & & & & & & & & $\square$ & & & & $\square$ & & & a & & E & & & & & & \\
\hline B. soulptitie Cushman & & & & & O & & & & & & & & & & & & & & & & & & & & $\square$ & & & & & \\
\hline B. spp. & & & & & $\mathrm{Ol}$ & $\square$ & $\underline{5}$ & 10 & & $\square$ & & & & $\square$ & & & 0 & & $\square$ & 10 & D & 0 & & 는 & 可 & & & 0 & & $\square$ \\
\hline B. $[?] \mathrm{sp}$. & & $\mathrm{C}$ & & & 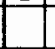 & & 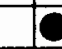 & & & & & $\square$ & & $\square$ & 8 & & 1 & & & & & $\square$ & & & & a & & & & \\
\hline Cancris cosoaenaie Cushman & & & & & 0 & & & & & & & & & T & & & $\square$ & & & & & & & & & & & 0 & & Z \\
\hline $\begin{array}{l}\text { FIGLARE B DISTRIBUTION OF FORAMINIFERA } \\
\text { FROM THE STRATA OF CASCADE MEAD }\end{array}$ & $\begin{array}{c}\text { FAUNULE } \\
\text { OA } \\
\text { ZONULE }\end{array}$ & 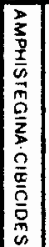 & 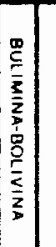 & 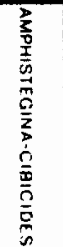 & & & & 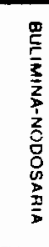 & & & & & 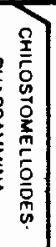 & 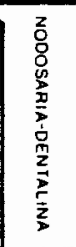 & 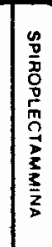 & 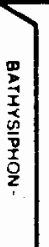 & 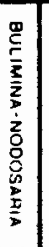 & & 兽 & & & 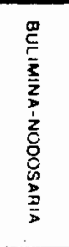 & & & & & & & & \\
\hline
\end{tabular}




\begin{tabular}{|c|c|c|c|c|c|c|c|c|c|c|c|c|c|c|c|c|c|c|c|c|c|c|c|c|c|c|}
\hline \multirow{2}{*}{\begin{tabular}{ccc} 
& \multicolumn{3}{c}{ LEGEND } \\
SPECIMENS/SAMPLE & $1-2$ & 0 \\
& $3-5$ & 0
\end{tabular}} & SECTION & \multicolumn{18}{|c|}{ NESKOWIN CREEK } & \multicolumn{2}{|c|}{$\begin{array}{l}\text { CASCADE } \\
\text { MP }\end{array}$} & {$\left[\begin{array}{l}\text { THAEE } \\
\text { RKS }\end{array}\right]$} & \multicolumn{4}{|c|}{ SALMON R. } \\
\hline & \multirow{2}{*}{$\begin{array}{l}\text { PSU } \\
\text { LOCALITY } \\
\text { NUMBER } \\
\end{array}$} & & & & & \multirow{2}{*}{\begin{tabular}{|c|c|c|c|} 
& 1 \\
0 \\
0
\end{tabular}} & \multirow{2}{*}{\begin{tabular}{l|l}
3 \\
0
\end{tabular}} & & & \multirow{2}{*}{$\stackrel{b}{\Xi}$} & \multirow{2}{*}{$\frac{3}{2}$} & $\underline{0}$ & ? & & & 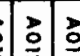 & 官 & & & 0 & 하이 & & & & & 히 \\
\hline SPECIES & & & & & & & & & & & & & $\bar{v}$ & & & & & & $\bar{\alpha}$ & $\vec{\sim} \mid \vec{\infty}$ & & & $\bar{\omega}$ & & & $\bar{v}$ \\
\hline Canoris joaquinenais Smith & & & & & & & & & & & & & & & ] & & $\square 0$ & & & ㅁ] & & $\mathbf{E}$ & & 01 & & 0 \\
\hline c. [?] ap. & & $\square$ & 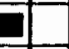 & & & $\square$ & & & & & & $\square$ & $\square$ & & & & & L & ] & & & & & & & \\
\hline Cassidulina globosa Hantken & & & 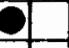 & & & 口्C & L & $\square$ & $\square$ & 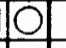 & & $\square$ & $\square$ & $\square$ & 9 & 10 & 01 & $\square$ & 1 & 0 & of & $\square[$ & & 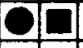 & & E \\
\hline Ceratobulimina alasanensis Cushman a Harris & & & & & & & & & & & 무 & $\square$ & & E & 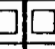 & $\square$ & $\square$ & E & 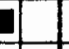 & & & & & & & \\
\hline Chiloguambelina oubansia (Palmer) & & & & & & 든 & ] & & & & & & & & & & - & & & & & E & & & & \\
\hline Chitostomelloides cooenica cushman & & & 1 & & {$[0$} & & D & O) & O & & Q & & D & & $\square$ & & $\square$ & $\mathrm{O}$ & 0 & & & $\square$ & $\square$ & $\square \square$ & & 0 \\
\hline c. oviformis [?] (Sherborn \& Chapman) & & & & & & & & & & & & & & & & $\square$ & I & 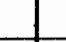 & & & & & & 11 & 1 & \\
\hline c. sp. & & & & & $\square$ & & & & 口마 & & & & $\square$ & & 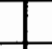 & D & $\square$ & 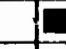 & 1 & & & & & $\square \square$ & $\square$ & \\
\hline Cibioideg ouahmani Nuttall & & & & & & $\square$ & & & 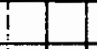 & & & & & & $\sqsupseteq$ & & I & & & & & & & & 四 & $\square$ \\
\hline c. felix Martin & & & & & & & $\square$ & $\square$ & a & & & $\bar{Q}$ & $D$ & & ] & $\square[$ & $\square$ & & & $\square$ & & $E$ & & & & \\
\hline c. cf. c. felix Martin & & & & & $\square$ & & & & & & & $E$ & & & & $\square$ & $\square$ & [ & ] & $\square$ & & 0 & & & & \\
\hline c. laimingi Mallory & & & & & & & & & 마 & & & & & & & -1 & I & & & & & & & & & \\
\hline c. Laurioae Mallory & & & & & & & & & & & & & & & & & $\square$ & & & E & & & & ㅁ. & & O) \\
\hline c. Laurieae [?] Mallory & & $\mathrm{O}$ & & & & & & & & & & & & & & & T & & & & & & & & & \\
\hline c. lobatue (d'oxbigny) & & & ב & & & 口 & & & & & & & & & & & $\square$ & & & & & & & & & \\
\hline c. momastersi Beck & & $\square$ & Oप & ]) & & & & & & & & & & & & & & & & & & & & & & O \\
\hline c. momastarai $[2]$ Beck & & & & & & & & & & & & & & & & & & & & $\square$ & & & & & & \\
\hline c. ouachitzensia var. athambrensio Smith & & & $\square$ & & & & & & & & & & & & & & & & & & & & t & & D & \\
\hline c. pachyderma (Rzehak) & & & & & & & & & & & & & & & & $\square$ & a & & & & & E & & & & 0 \\
\hline c. ef. c. praecursorius (schwager) & & & & & $\square$ & & & & & & & & & & & & & & & & & $\underline{\square}$ & & & & \\
\hline c. peeudoungerianue (Cushman) & & & & & O & OC & & alac & Ot口 & & $\square$ & & & & 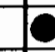 & & & & & & & & & & & \\
\hline c. cf. c. pesudoungerianus (Cushman) & & & & & & & & $\square$ & & & & & & & & & & & ] & & & & & & & \\
\hline c. paeudoune ltoratorffi Cole (?) [of Mallory] & & & & & & L & & & & & & & & & & & & & & & & & & & & \\
\hline c. eandiegensis Cushman G. M. A. Hanna & & & & 4 & & & & & & & & & & Of & 1 & & Ol & & & 0 & & & & & & \\
\hline c. cf. c. oandiegensis Cushman \& M. A. Banna & & & & & & 口] & & & & & & 도 & & $\square$ & t & & & & & O & & & & & Q & \\
\hline c. spiropunotatue Galloway \& Morrey & & & & & & & & & & & & & & & & 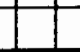 & 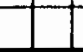 & & & & & & e & 口 & & 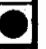 \\
\hline $\begin{array}{l}\text { FKUIRE } 8 \text { DISTRIBUTION OF FORAMINIFERA } \\
\text { FROM THE SIRATA OF CASCADE HEAD }\end{array}$ & $\begin{array}{l}\text { FAUNULE } \\
\text { OR } \\
\text { ZONULE }\end{array}$ & 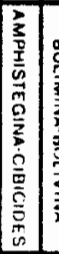 & 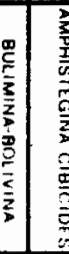 & 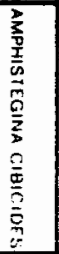 & & & & 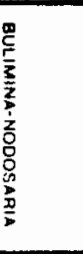 & & & 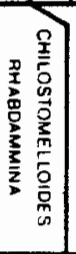 & 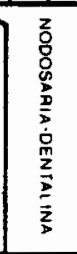 & 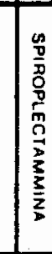 & 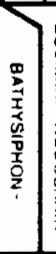 & 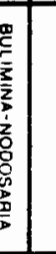 & & $\frac{0}{\frac{0}{2}}$ & & 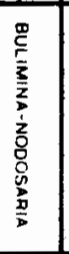 & & 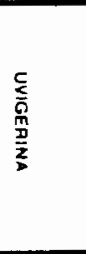 & & & 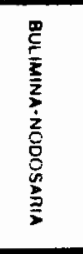 & & \\
\hline
\end{tabular}




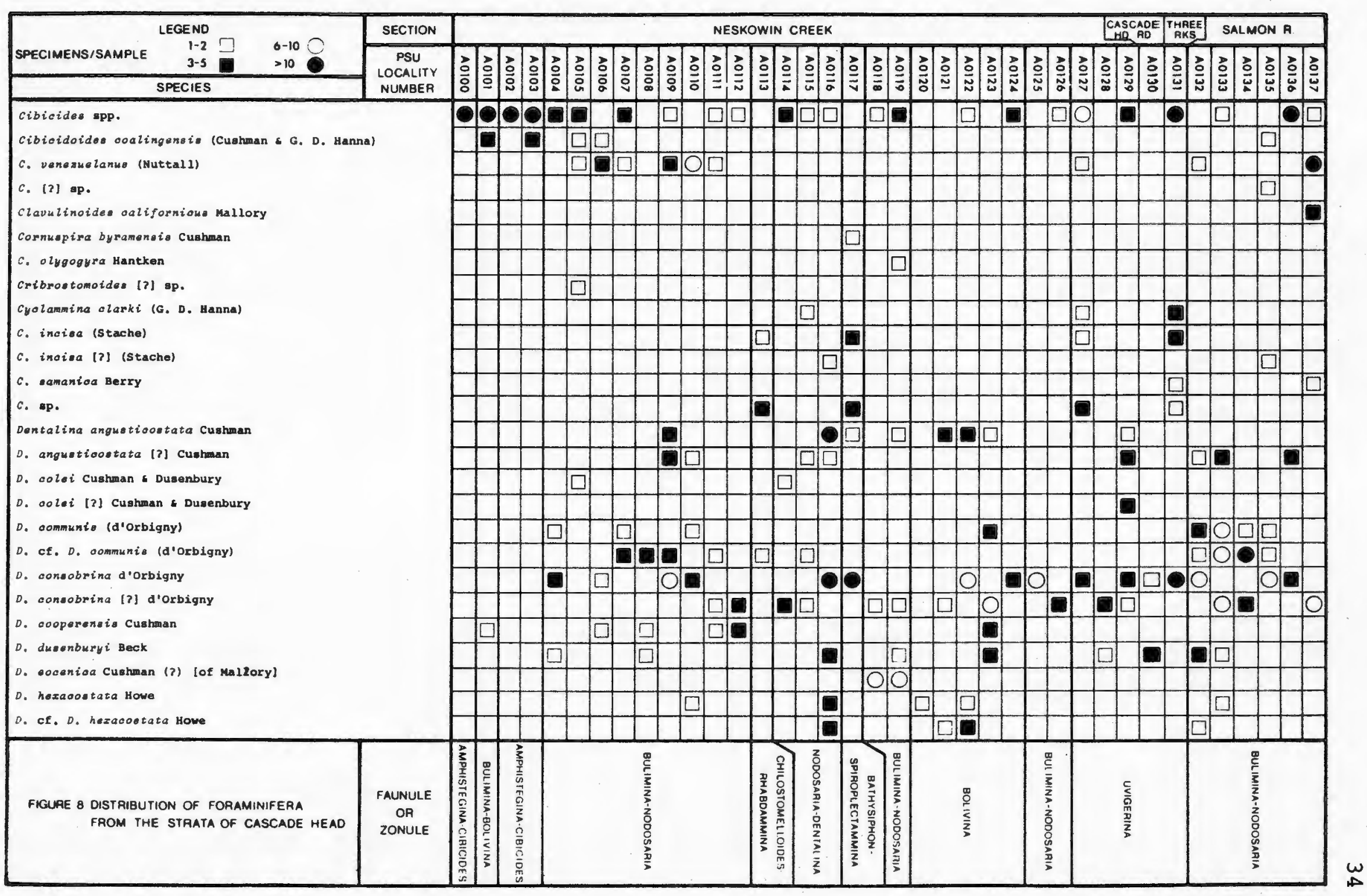




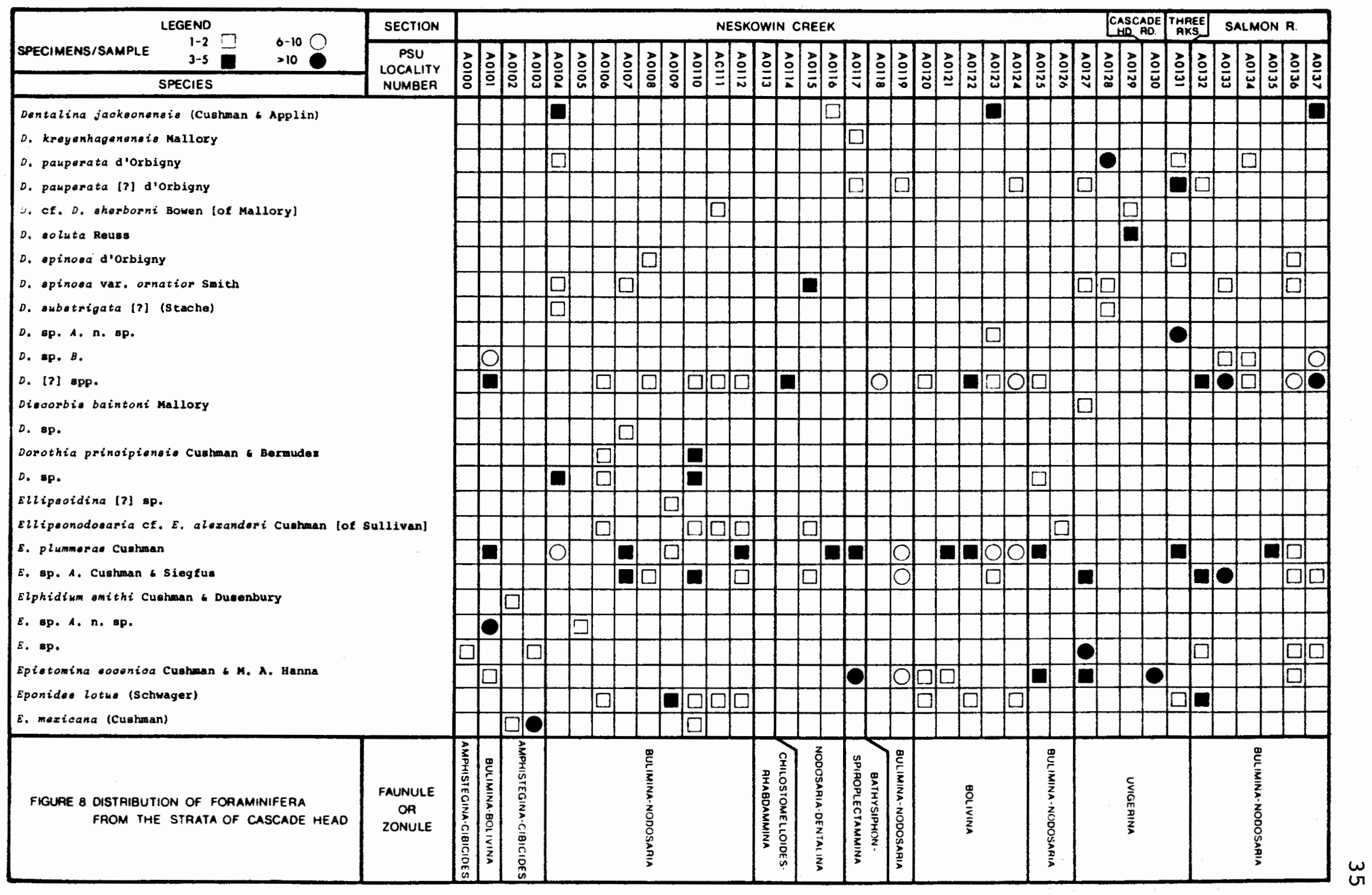




\begin{tabular}{|c|c|c|c|c|c|c|c|c|c|c|c|c|c|c|c|c|c|c|c|c|c|c|c|c|c|c|c|}
\hline LEGEND & SECTION & & & & & & & & & ESKO & SWIN & CAEEK & & & & & & & & & $\begin{array}{ll}\text { CAsC } \\
\mathrm{CAO}\end{array}$ & & RAES & & LMON & & \\
\hline $\begin{array}{lll}\text { SPECIMENS/SAMPLE } & 3-5 & >10 \\
\end{array}$ & $\begin{array}{l}\text { PSU } \\
\text { LOCALITY }\end{array}$ & 흥 & & 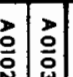 & 임 & 客 & 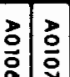 & 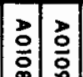 & 索咅 & 祘 & 흫일 & $\frac{0}{2}$ & $\frac{2}{\partial}$ & $\frac{z}{3}$ & 亘 & $\underset{0}{0}$ & $\frac{n}{2}$ & \begin{tabular}{lll}
3 \\
\hdashline \\
0
\end{tabular} & $\Rightarrow$ & $\frac{3}{3}$ & & 玆 & 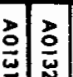 & $\frac{3}{3}$ & & $\frac{0}{\omega}$ & $\frac{3}{0}$ \\
\hline SPECIES & & & & & & & & & & & & & & & & & & & & & & & & & & & \\
\hline Eponides mexioana (3) (Cushman) & & $\mathrm{a}$ & & & & $\square$ & & $\square$ & & & & & & & & & & & & & & & 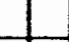 & 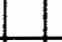 & & & \\
\hline E. umbonata (Reuss) & & & & & & & $\square$ & $\square$ & a & (口) & & $\square[$ & G & & & (口) & 0 & $\square$ & $\square$ & & $\square$ & 0 & a & 0 & & & 0 \\
\hline E. umbonata [?] (Reuss) & & & & & & & & & & & & {[} & ] & & & & & & & $\square$ & & & & & D & & \\
\hline E. valtonensio [?] Applin \& Jordan & & $\square$ & & & & & & & & & & & & & & & & & & & & & & & & & \\
\hline s. spp. & & Q & $\square[$ & $\square \square$ & & & $\square$ & & & & & {[} & ] & & & & & & Q & & & & & O & & $\square$ & 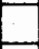 \\
\hline Gaudryina cf. G. laevizata Pranke lof Malloryl & & & & & & & & & & & & & & & & & & & & & & & & & & & 0 \\
\hline c. [2] sp. & & & & & & & & $\square$ & & & & & & & & & & & & & & & & & & & \\
\hline Clobigerina oiperoenois anguatiumbilioata Boll1 & & & & & & & & $\square$ & & & & & & & & & & & & & & & E & D & $\underline{\mathbf{a}}$ & & L \\
\hline O. oiperoensio anguatiumbilicata (z) Bolli & & & & & & & & & & & & & & & & & & & & & & & & & & $\square$ & \\
\hline a. cf. o. oonoinna Reuse & & & & & & & & D & 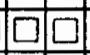 & & {[} & & & & $\square$ & $\square$ & & & & & & & & & & & 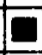 \\
\hline c. decepta Martin & & & & & & & & $\square$ & & & & & & & & & & & & & & & & & & 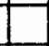 & \\
\hline c. oucehitaensia Howe \& Wallace & & & 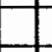 & & & $\square$ & & 口 & & & & & & & 口 & 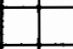 & $E$ & ב & & - & & & 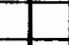 & & & 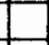 & $\square$ \\
\hline o. of. G. ouachitaenaid Howe \& Wallace & & & $\square$ & & D & & $\square$ & & & & & $\square$ & & & 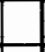 & E & 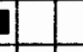 & & a & $\square$ & & & E & & & & E \\
\hline a. prasbulloidas Blow & & & & & & & & & & & & & & & $\square$ & & & & & & & & & & & & \\
\hline G. prasappis Blow & & & & & & & & & & & & E & $2[0$ & & & $\square$ & 1 & & & L & & & & & & L & \\
\hline G. primitiva (Finlay) & & & & & & & $\square$ & & & & & & & & & & & & & & & & & & & & \\
\hline c. cf. c. yeguaensis Weinzierl \& Applin & & & & & & & & & & 마 & & & & & & & & & & & & & & & & & \\
\hline c. spp. & & $\mathrm{O}$ & Q & & $\square$ & & $\square[$ & 0 & (a) & O & $\mathrm{OO}$ & 0 & 0 & $\bar{\square}$ & $\square$ & $\square$ & & ofla & $\square$ & $\square$ & a & $E$ & $\square$ & O & & $\square$ & O \\
\hline Globigerinatheka index (Finlay) & & & & & & & & & & & & $\square$ & & & + & 1 & & & & 1 & & & & & & & \\
\hline Jlobobulimina pacifioa Cushman & & & & & & & 0 & & & $\square$ & DE & D & $\square$ & & $\square$ & 뭉 & a & & & & D & & & & & & \\
\hline c. pacifioa var. somprease Hornaday & & & & & & & & & $\square$ & & & & & & $\square$ & & & & & & the & & & & & & \\
\hline c. [?] sp. & & & & & & & & & & & ㅁ & & & & & 10 & 0 & & & & Q & & & & & & \\
\hline Globorotalia cf, $G$, oerroasulensio (Cole) & & & 0 & & & & & & & & & & & & & & & & & & & & & & & & 0 \\
\hline 3. [7] spp. & & & 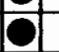 & & & & 口 & & & a & 口 & & $\square$ & 0 & & $\square$ & $\square$ & & $\square$ & $\square$ & & & $\square$ & & & $\square$ & [a \\
\hline sloborotaloides turgide (Finlay) & & & & & & & & $\square$ & & & & & & & & & & & & & & & & & & & \\
\hline (i. sp. [7] lof Mcreel \& Lipps] & & & & & & & & & & & & & & & & & & & $\square$ & & & & & & & & \\
\hline $\begin{array}{l}\text { FIGURE Q. OSTRIBUTION OF FORAMINIFERA } \\
\text { FROM THE STRATA OF CASCADE HEAD }\end{array}$ & $\begin{array}{c}\text { FAUNULE } \\
\text { OA } \\
\text { ZONULE }\end{array}$ & 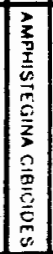 & 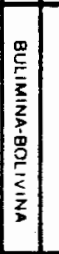 & 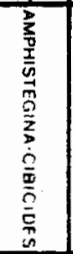 & & & & 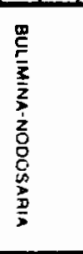 & & & 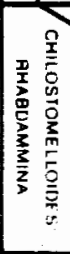 & 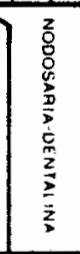 & & 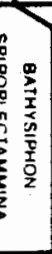 & 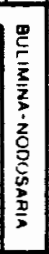 & & $\begin{array}{l}\frac{0}{2} \\
\frac{0}{5} \\
\frac{5}{2} \\
\frac{1}{2}\end{array}$ & & 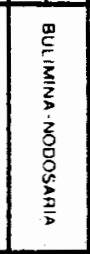 & & 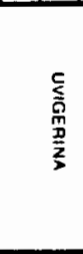 & & & & 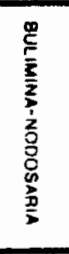 & & \\
\hline
\end{tabular}




\begin{tabular}{|c|c|c|c|c|c|c|c|c|c|c|c|c|c|c|c|c|c|c|c|c|c|c|c|c|c|c|c|c|c|c|c|c|}
\hline LEGEND & SECTION & & & & & & & & & & NESKC & oWIN & CAEE & & & & & & & & & & & & 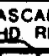 & & $\begin{array}{l}\text { THAEE } \\
\text { AKS }\end{array}$ & & ALLMC & ON F & & \\
\hline SPECIMENS/SAMPLE $\quad 3-5 \quad 210$ & PSU & 2 & & & & & & & 3 & 3 & 3 a & b & & 吾 & 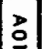 & & 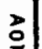 & 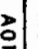 & & & ? & & & ? & 它 & 羊 & & & & & $\underline{a}$ & \\
\hline SPECIES & & $\overline{8}$ & & (5) & & & ة & 5 & $\overline{0}$ & & $\equiv \bar{n}$ & $\vec{\omega} \mid \vec{E}$ & $\overline{4}$ & $\bar{\alpha}$ & $\bar{z}$ & $\bar{\infty}$ & $\overline{0}$ & ة & $\tilde{N}$ & & $\Sigma$ & & & $\bar{\infty}$ & พิ & ة & $\overline{\underline{w}}$ & $\bar{\omega}$ & & 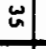 & 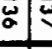 & \\
\hline Guttulina franksi cushman t Ozava & & & & $\square$ & & & & & & & & & & & & & 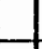 & & & & 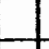 & 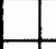 & & & & & & & & & & \\
\hline c. irregularia (d'orbigny) & & & (7) & & & & & & & & & & & & & & & & & & & & & $\square$ & & $\square$ & & $\mathbf{a}$ & & & 5 & \\
\hline c. $[7]$ sp. & & & & & & & & a) & & & & & & & & & & & & & & & & & & - & & $t$ & & & - & \\
\hline cypoina globula [r] (Reuss) & & & & & & & & & & & & & & & & & & & & & & & & & & & & $\square$ & & & & \\
\hline Cypoidina sondoni (Cushman a Schenck) & & & $\square$ & $\square$ & & & & & & $\square$ & & 느 & & & & 口 & 0 & & (5) & a & 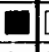 & $\square$ & L & {[]} & & & & 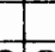 & & & 7 & \\
\hline a. orbicularia var. planuta Cushman & & $\square$ & & & $\square$ & $\square$ & Q & $E$ & $\square$ & $\square$ & $\square$ & & & 2 & $\square$ & & $\square$ & ㄱ. & ] & $\square$ & a & $\square$ & & 0 & & P. & 田 & 90 & & ㅁ] & al & \\
\hline ¿. ooldanii d'oxbdgny & & & & & & $\square$ & & & & & & & & & & & & & & $\square$ & & & & & & & & O & & & & \\
\hline i. soldanii $(7)$ d'orbigny & & I & $\square$ & & & & & & & & & & & & & & & & & & & & & & {$[\mathrm{a}$} & & & & & & & \\
\hline 3. soldanii var, ootocamerata Cuahman \& G. D. & inna & & & & & & & $\underline{5}$ & ] & & & & & & 口 & $\square$ & & 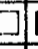 & ב & & & & & & & & & & & & $E$ & \\
\hline o. of. G. Coldanii var. ootocamerato Cushmen s & D. D. Hanna & & & & & & & & & & & & & & & & & & & & & Q & & & & & $\square$ & & & & & \\
\hline c. spp. & & Q & & & & a & & 可 & $\square$ & & & 믄 & & & & Q & & ב & ] & $\square$ & & & & 0 & (0) & 미 & & ] & & 미 & _. & \\
\hline Haplophragmoides latiesimiauturalis Ssith & & & & & & & & ] & $\square$ & & $\square$ & & & & $\square$ & & $\square$ & & I & - & & & 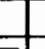 & $\square$ & $\theta$ & & O & 1 & & & - & \\
\hline s. sp. & & & & & & & & $E$ & & {[} & $\square$ & & $\square$ & & & & & & & & & & 가 & & & & & & & & & \\
\hline Ayperammina [?] sp. & & O & & & & $\square$ & & & & & & & & & & $\square$ & & & & & & & & & & & & & & & & \\
\hline Karreriella ahapapotenaia var. monumentensis $\mathrm{M}$ & $10 x y$ & & & & & & & & & 만 & $\square$ & 다 & & & $\square$ & & & & & & & & & & - & & 1 & 1 & & & - & \\
\hline K. Elongata Mallory & & & & & & & & & $\square$ & & & & & & & & & & & & & & & & & & & 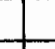 & & & {[} & \\
\hline x. mediaagkaenaio Mallory & & & & & & & & & & & $\square$ & & & & & & & & & & & & & & & & (9) & $\square$ & & & & \\
\hline$x,[7]$ sp. & & $\square$ & & & & & & E & $\square$ & $\square$ & $\square$ & & & & & & & & & & & & & & & & O & & & & & \\
\hline Lagena acuticosta Reuss & & & 9 & & & $\square$ & & & & & & & & & & & $\square$ & & & & & & & & a & & & E & & & $\underline{\square}$ & \\
\hline L. Amphora Reuar & & & ㅁ] & & & & & & & & & & & & & & & & & & & & & & & & & $\Xi$ & & & 드 & \\
\hline L, beoki Sullivan & & & & & & & & & & & & & & & & 미 & a & & & $\square$ & & & & & & & & & & & & \\
\hline L. Costata (Williamaon) & & & & & $\square$ & & & & & & & & $\square$ & & & & & & & & & $\square$ & & & & & & & & & & \\
\hline L. aostata [?] (Williamoon) & & & & & & & & 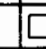 & ]) & & & & & & & & & & & & & & & & & & $\underline{\square}$ & 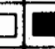 & & & & \\
\hline L. hexagona (Hilliamaon) & & & & & & & & & $\square$ & & & & & $\square$ & & & & & & & & & & & & & & & & & [ & \\
\hline L. marginata (Walker \& Boys) & & & $\square$ & & & & & & & & & & & & & & & & & & & & & & & & & & & & & \\
\hline E. vulgaris williamson & & & & & & & & & & & $\square$ & & & & & & & & & & & & & & & & $\square[$ & ] & & & & \\
\hline $\begin{array}{l}\text { FIGURE } 8 \text { DISTRIBUTION OF FORAMINIFERA } \\
\text { FROM THE STAATA OF CASCADE HEAD }\end{array}$ & $\begin{array}{c}\text { FAUNULE } \\
\text { OR } \\
\text { ZONULE }\end{array}$ & 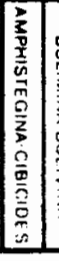 & 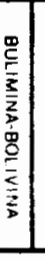 & 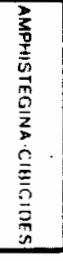 & & & & 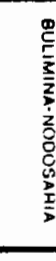 & 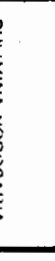 & & & 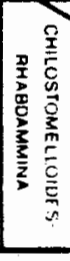 & & 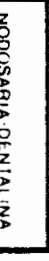 & 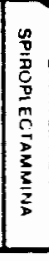 & 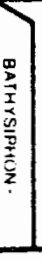 & 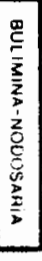 & & & & & 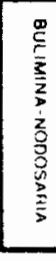 & & & 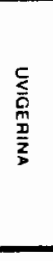 & & & & $i^{i}$ & 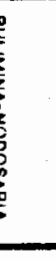 & & \\
\hline
\end{tabular}




\begin{tabular}{|c|c|c|c|c|c|c|c|c|c|c|c|c|c|c|c|c|c|c|c|c|c|c|c|c|c|c|}
\hline LEGEND & SECTION & & & & & & & & & NESKOI & DWIN CF & CAEEK & & & & & & & & CAsc & & $\begin{array}{l}\text { THREE } \\
\text { AKS } \\
\text { S }\end{array}$ & & ALMON & & \\
\hline SPECIMENS/SAMPLE $\quad 3-5$ - $>10$ & $\begin{array}{l}\text { PSU } \\
\text { LOCALITY }\end{array}$ & 总 & & & $\overrightarrow{0}$ & \begin{tabular}{|l|l|}
0 & 0 \\
0 & 0
\end{tabular} & $\vec{a}$ & $?$ & 3 & 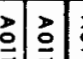 & 家至 & 히링 & 豆 & 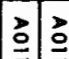 & 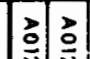 & $\underline{0}$ & $\overrightarrow{0}$ & & 희 & & 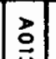 & & 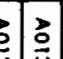 & 이 & & 恶 \\
\hline SPECIES & & |" & & & & & & & & & & & & & & & & & & & & & & & & \\
\hline Lentioulina cf. L. convargena (Bornemann) lof & $110 \mathrm{xy}$ & & & & & $\square$ & & & & & & & & & & & & & & & & & & & & \\
\hline Loxos tomum applinae (Plummer) & & & & & & & & & & & & & & & & & & & & & & [ & & $\mathrm{O}$ & & $\square$ \\
\hline Marginulina adunca (Costa) & & & & & D & $\square[\square$ & & & & $\square$ & & $\square$ & & & & & & & & & & & 0 & $\square$ & & \\
\hline N. adunca [?] (Costa) & & & & & & & & & & & $\square$ & & & & & & & & & & & & & & & \\
\hline M. cf. N. alaaneneis Nuttall & & & & & & 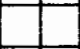 & & & D & & & & & & & & & & & & & & & & & \\
\hline N. eximia Neugeboren & & & & & $\mathrm{D}$ & & & & & & & & & & & & & & & & & & $\square$ & & & \\
\hline M. cf. M. eximia Neugeboren & & & & & & & & $\square$ & & & & & & & & & & & & & & & & & & \\
\hline N. holmesi Gaston & & & & & & & a & & & & & & & & & & & & & & & & & & & \\
\hline 4. huneri [2] Howe & & & & & & & & & & & & & & & & & & & & & & $\underline{E}$ & ] & & & \\
\hline M. cf. N. inoonspioua Husaey lof sullivan] & & & & & & & & & & & & & & & & & & & & & & & {[} & & & 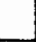 \\
\hline M. munda (?] Cushraan & & & & & & & & & & & & & & & & & $\square$ & & & & & & & & & \\
\hline M. ragulario d'orbigny & & & & & & & & & & & & & & & & & & & & & & & & & & {[]} \\
\hline M. Bubbullata Hantken & & & & & $\mathrm{O}$ & $\square$ & & $\square$ & a & & & $\square$ & & & & & $\square$ & & & & & & $\mathbf{E}$ & $\square$ & & \\
\hline N. sp. & & & & & $\square$ & & & & & $\square$ & & & & & & & $\square$ & & & & & & & & & L \\
\hline .4. [2] ap. & & & & & & & & & & D & 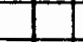 & 5 & & & & -1 & 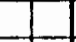 & L & & & & 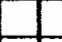 & & & & $\square$ \\
\hline Martinotzialla eocenioa Cushan + Boxaudes & & & & & & & & $\square$ & & & & $\square$ & & & & & & & & & & & & & & \\
\hline *. [?] ep. & & & & & & & & & & & & & & & & & & $\square$ & & & & $\square$ & & & & \\
\hline Massilina decorata Cushan & & & & & & & $\square$ & & & & & & & & & & - & & a & & & & & & & \\
\hline Modogenerina adolphina (d'Orblgny) & & & & & {$[-1$} & & $\square$ & & & & $\square$ & E & & & & & 9 & & & $\square$ & & & E & O & & \\
\hline ג. cf. N. bradyi Cushman & & & & & 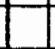 & & & & & $\square$ & & & & & & & $\square$ & & & & & $\square$ & & & & \\
\hline N. Kroasenbergensis (Gumbel) & & & & & $\square$ & & & $\square$ & & & & & & $\square$ & & & & & & & & DE & 10 & & $\square$ & \\
\hline N. Lepidu Za (Schwager) & & & & & & & & $\square$ & & & & & $\square$ & $\bar{\square}$ & $\square$ & 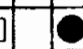 & t & & 0 & 10 & & 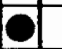 & & $\square$ & 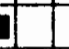 & \\
\hline N. Lepidnla [2] (Schwager) & & & & & & & $\mathrm{O}^{2}$ & & & & & & & & & & & & & & & & & & & \\
\hline s. app. & & & & & & & & & & 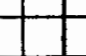 & & \begin{tabular}{|l|l|l}
$\square$ & \\
\end{tabular} & & & & 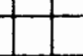 & 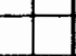 & $\square$ & & & & & O & $\square \square$ & & \\
\hline Nodosaria arundinsa Schwager & & & & & $\mathrm{O}$ & & & olo & 0 & a & & 0 & 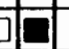 & & & & 0 & a & to & a & & & & a & ) & 0 \\
\hline N. arundinea [?] Schuager & & & & & & & & & & & 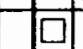 & 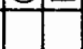 & & 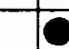 & & & & & & & & & & & & \\
\hline $\begin{array}{l}\text { FIGURE } 8 \text { DISTRIBUTION OF FORAMINIFERA } \\
\text { FROM THE STRATA OF CASCADE HEAD }\end{array}$ & $\begin{array}{c}\text { FAUNULE } \\
\text { OR } \\
\text { ZONULE }\end{array}$ & 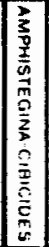 & 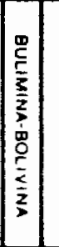 & 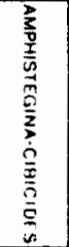 & & & & 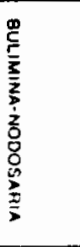 & & & 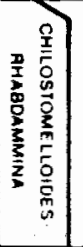 & 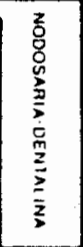 & 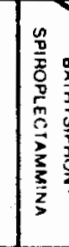 & 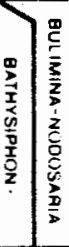 & & 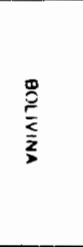 & & 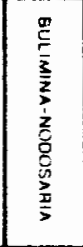 & & 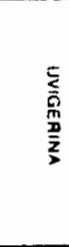 & & & & 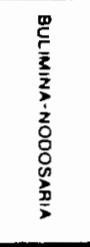 & & \\
\hline
\end{tabular}




\begin{tabular}{|c|c|c|c|c|c|c|c|c|c|c|c|c|c|c|c|c|c|c|c|c|c|c|c|c|c|c|c|c|c|c|c|c|}
\hline $\begin{array}{l}\text { LEGEND } \\
1-2\end{array}$ & SECTION & \multicolumn{22}{|c|}{ MESKOWIN CREEK } & \multicolumn{5}{|c|}{ 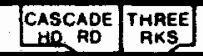 } & \multicolumn{4}{|c|}{ SALMON A. } \\
\hline SPECIMENS/SAMPLE $\quad 3-5=>10$ & PSU & 2 & & & 8 & & & & 2 & 8 & & & 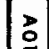 & & 8 & 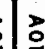 & & 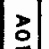 & 3 & & & & & & $\overrightarrow{0}$ & b & & & & & 의응 & \\
\hline SPECIES & & 各 & & & & & & & & & $\equiv \overline{\bar{N}}$ & & $E$ & & & & & $\overline{0}$ & $\underline{\underline{\Sigma}} \overline{2}$ & $\vec{U} \mid \bar{\omega}$ & & $\bar{u}$ & & $\bar{\Phi}$ & 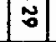 & 㟧 & $\underline{\underline{\underline{\omega}}}$ & & $\bar{\omega}$ & $\mid \begin{array}{c}\mid \vec{\omega} \\
\end{array}$ & 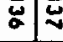 & 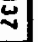 \\
\hline Hodosaria shirana Cushman I Stone & & & & & & & & & & & & & & & 口 & & $\square$ & & & $\square$ & & & & & & & & & & & 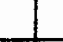 & \\
\hline N. delioige Martin & & & & & 口 & & & & & & & & & $\square$ & & & & & & & & & & & & & & & & & & \\
\hline N. cf. N. deliciae Martin & & & & & $E$ & & & 更 & & & & & & & & & & & & $\square$ & & & & & & & & $\bar{C}$ & & at & O & \\
\hline H. ewazdi Reuss & & & & & & & 0 & E & 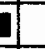 & 10 & DL & & & DI & ] & {[} & a & & 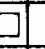 & & & $\square$ & $\mathbf{0}$ & $\square$ & & & $\square$ & & a & & & \\
\hline N. ewazdi [?] Reuss & & & & & & & & & & & & & & & & & & D & & & & & & & $\square$ & & & ] & & & & \\
\hline N. (?) gyrata Mallory & & & & & a & & & & & & & & & $\square$ & & & & & & & & & & & & & & & & & & \\
\hline N. (2) gyrata Mallory n. var. & & & & & E & & & & & & & & & & & & & & & & & & & & & & & & & & & \\
\hline N. holeorioa Schwager & & & & & & & $\square$ & & & a & & & & {[} & ] & & & & & & & & & & & & & & & & & \\
\hline N. cf. A. hozaerica Schwager & & & & & & & & {[} & $\bar{C}$ & & & & & & E & & & & & 0 & & & & & & & O & & & & & \\
\hline N. latejugata Gumbel & & O & & O & & D & & & & & & & & & & & & & & & & & & & & & & & & & & \\
\hline N. Longiscata d'orbigny & & & & & & & & & & & & & & & & & & & & $\square$ & & & & & & & & & & & & \\
\hline v. pyrula d orbigny & & & & & E & & & 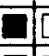 & & & & & & & & & & & & & & Of & & & & & 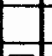 & & 20 & & Of & \\
\hline N. pyruta [z] d'oxbigny & & . & & & & & & a] & ] & E & $E$ & & & & & & & & & 1 & & $F$ & & & & $\square[$ & $\square$ & & & & $E$ & 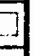 \\
\hline N. sp. [of Mallory] & & & & & & & & & & & & & & & & & & & & & & & & & & & & & & & $\underline{I}$ & 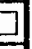 \\
\hline N. [?] spp. & & $\square$ & $\square$ & E & $\square$ & & a & 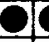 & E & 0 & 0 & E & 0 & $\square$ & E & & $\square$ & as & o[ [ & ] & O & 口 & & O & t & Of & 0 & & 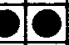 & D & 0 & \\
\hline Nonion durhami Mallory & & & & & & & $\mathbf{a}$ & & $E$ & & & & & & & & & & & & & $\mathrm{~L}$ & & & & 1 & 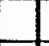 & & & & & \\
\hline N. durhami [?] Mallory & & & & $\square$ & & & & & & & & & & & & & & & & & & & & & & & & & & & & \\
\hline N. planatum Cushman o Thomas & & & a & & & & & & & & & & & & {[} & {$[\square$} & & D & 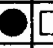 & {$[\mathbf{a}$} & & $\square$ & L & & & $\square$ & & & & & $E$ & 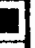 \\
\hline N. planatum [?] Cushman a Thomas & & & 0 & & & & & & & & & & & & & & & & & & & & & & & & a & & & & {[]} & \\
\hline N. ap. A, n. ap. & & & & & & & & & & & & & $\square$ & & & & 0 & (口) & $\partial$ & $\square$ & & & & $\square$ & & & & & & & & \\
\hline w. sp. & & $\mathbf{a}$ & & & & & & & & & & & & & & $\square$ & & & 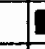 & 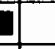 & & & & & $\square$ & & & & $\square$ & & {[} & 2 \\
\hline Parrelza tanuigarinata (Cushman \& Slegfua) & & & & & & E & $\square$ & & {[} & & -1 & & & $\square$ & $\square$ & & & & & 0 & & Q & & & & & & & & & & \\
\hline Planularia markleyana church & & & & & & & & & & & & & & & & & & & & & & & 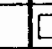 & & & & & & & & & \\
\hline P. Cf. $P$, ouqohitaznsia Howe s wallace lof Mal & & & & & & & & & & & & & & & & & & & & 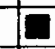 & & & & & & & & & & & & \\
\hline P. cf. P, truncana (Gumbel) & & & & & & & & & & $\square$ & & & & & & & & & & & & & & & & & & & & & & \\
\hline p. [2] ep. & & & & & & & D & & & $\square$ & & & & & & & 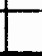 & & & & & & & & & & & & & & {[} & \\
\hline $\begin{array}{l}\text { FIGURE } 8 \text { DISTRIBUTION OF FORAMINIFEAA } \\
\text { FROM THE STAATA OF CASCADE HEAD }\end{array}$ & $\begin{array}{c}\text { FAUNULE } \\
\text { OR } \\
\text { ZONULE }\end{array}$ & 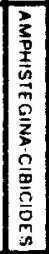 & \begin{tabular}{|l}
0 \\
0 \\
$\frac{0}{3}$ \\
2 \\
2 \\
0 \\
0 \\
0 \\
$\frac{2}{2}$ \\
2 \\
2
\end{tabular} & 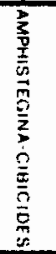 & & & & & 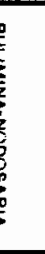 & & & & 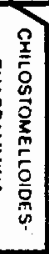 & 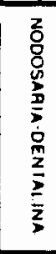 & & 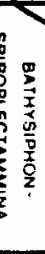 & 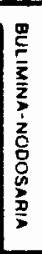 & & $\frac{1}{3}$ & & & 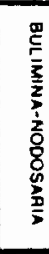 & & & 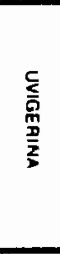 & & & & 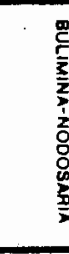 & & & \\
\hline
\end{tabular}




\begin{tabular}{|c|c|c|c|c|c|c|c|c|c|c|c|c|c|c|c|c|c|c|c|c|c|c|c|c|}
\hline LEGEND & SECTION & & & & & & & & NESK & OWIN C & CAEEK & & & & & & & & $\begin{array}{l}\text { ASCADE } \\
\text { AR RO } \\
\text { RO }\end{array}$ & THAS & & SALMOA & N R. & \\
\hline $\begin{array}{lll}\text { SPECIMENS/SAMPLE } & 1-2 . & 6-10 \\
& 3-5 & >10\end{array}$ & PSU & 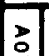 & & & 20 & & & & $\vec{a} \mid \overrightarrow{0}$ & 30 & 18 & & 3 & 3 & 03 & 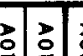 & $\Rightarrow$ & \begin{tabular}{|l|l|l|}
0 & 0 \\
0
\end{tabular} & 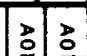 & a & & to & & \\
\hline SPECIES & $\begin{array}{l}\text { LOCALIYY } \\
\text { NUMBER }\end{array}$ & & 至 & 의 & 힌 & 安 & 5 & : & $\equiv \overline{\bar{N}}$ & $\bar{\omega} \mid \equiv$ & & $\bar{\nabla} \equiv$ & $\overline{\bar{\omega}} \mid \overrightarrow{0}$ & & & & & & & & & & & \\
\hline Planulina sp. & & & $\square$ & & & & & & & & & & & & & & & & & & & & & \\
\hline Pleotofrondioularia gracilia Snith & & & & & & & & & & & & & & & & & & a & & & & & & \\
\hline P. kerni cook & & & & & & & & & & & $\square$ & & & & & & & 1 & & & & & & \\
\hline P. oregononeia Cushman, stevart, I stewart & & & & & & & & & & & & & $\square$ & & & & & $\square$ & & $\square$ & & & & \\
\hline P. packardi var. multilineata cushman os simona & & & & & & & & & & & & & a & & $\square$ & & & & & O & & & & \\
\hline P. packardi var. packardi Cushman a Schenck & & & & & & & & & & & & & & & 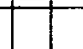 & & & $\square$ & & & & & & \\
\hline P. aff. P. paokardi var. paokardi Cushman s sc & & & & & & & & & & & & L & ] & & $\square$ & & & 且 & $\square[$ & (1) & & & & \\
\hline P. Esarai Cushman, stewart, E Stewart & & & & & & & & & & & & & & & $\square[$ & $\mathbf{a}$ & & $\square$ & $\square$ & & & & & \\
\hline P. vaughani Cushman & & & & & & & & & & & 0 & ot & ]田 & & 10 & 0 & & ( & $\square$ & & & & & \\
\hline P. vokeai Cuahman, stowart, stowart & & & & & & & & & & & \begin{tabular}{|l|l|l|} 
\\
\end{tabular} & te & 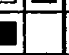 & & to & $\frac{10}{D}$ & {[} & C & & & & & & \\
\hline P. ap. [of Waaver a Weaver] & & & & & & & & & & & & $T$ & & $\square$ & 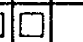 & & & T & 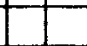 & & & & & \\
\hline P. ap. & & & & & & $\square$ & & & & & $\square$ & & & & D品 & ]国 & $\square$ & $\square$ & a & & & & & \\
\hline Plourostomezza aouta Hantken & & & & & $\square$ & $\square$ & D & 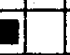 & $\square$ & & & $\theta$ & & & & & $\square$ & & & & & & & $\square$ \\
\hline P. Cf. P. aeuta Hantken & & & & & & & $\square$ & 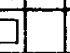 & & & & & & & & & & & & & & & & \\
\hline P. gredalensie cook & & & & & & & $\square$ & ] & & & & - & & & 1 & & E & L & & & & & & \\
\hline P. cf. P. gradalensis cook & & & & & & & & $\square$ & & & & & & & & & & & & & & & & \\
\hline P. paleocanioa Cushman & & & & & & & & $\square$ & & & & & & & & & & & & & & & & \\
\hline P. sp. & & & & & & $\square$ & $\square$ & ] & $\square$ & & $\square$ & & & & & & $\square$ & & & & & & & \\
\hline Pooudoglandulina coniac (Neugeboren) & & & & 10 & & & & 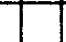 & & & & & & & & & & & & & $\square$ & & & $\square$ \\
\hline P. ovata (Cushman \& Applin) & & & & & & $\square$ & $\square$ & 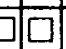 & $\square$ & $\square$ & 同 & $\square$ & & & $\square$ & (]) & & $\square$ & & & $\square$ & & & \\
\hline Paeudohastigerina miara (cole) & & & & & & & E & H & a & & & & & & & & $\mathbf{\theta}$ & & & & & & & E \\
\hline Pullenia cosenioa Cushman is siegfus & & & & & & & & & & & $\square$ & & & & & & & & & & & & & \\
\hline P. quinqualoba (Reuss) & & & & & a & & & & & & & - & & & & & & & & & $\square$ & D & & $\square$ \\
\hline P. salioburyi stewart a stewart & & & & & a & & & & & & & 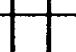 & & & & & & & & $\square$ & & & & \\
\hline P. вp. & & & & & & & & & & & $\square$ & & & & & & & & & & & & & \\
\hline P. [3] sp. & & & & & $\square$ & & & & & & 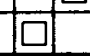 & 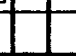 & & & & & & & & & & & & \\
\hline $\begin{array}{l}\text { FIGURE Q DISTRIBUTION OF FORAMINIFEFA } \\
\text { FROM THE STRATA OF CASCADE HEAD }\end{array}$ & $\begin{array}{c}\text { FAUNULE } \\
\text { OA } \\
\text { ZONULE }\end{array}$ & & 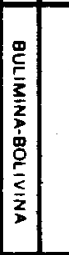 & 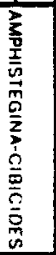 & & & 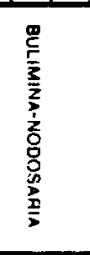 & & & 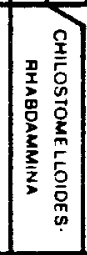 & 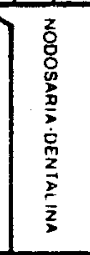 & 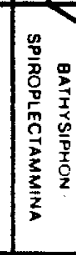 & 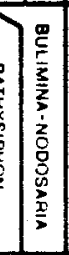 & & 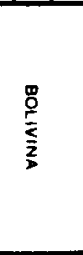 & & 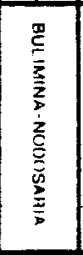 & & 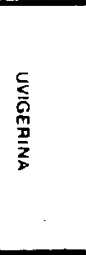 & & & 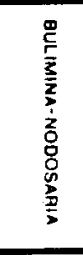 & & \\
\hline
\end{tabular}




\begin{tabular}{|c|c|c|c|c|c|c|c|c|c|c|c|c|c|c|c|c|c|c|c|c|c|c|c|c|c|c|c|c|c|c|c|c|}
\hline LEGEND & SECTION & & & & & & & & & & NESK & Kov & $\overline{\operatorname{INC}}$ & SREEK & & & & & & & & & & & $\operatorname{CASCA}_{H B}$ & & $\begin{array}{l}\text { PAAE: } \\
\text { RKS }\end{array}$ & & & MON & & \\
\hline SPECIMENS/SAMPLE $\quad 3-5=10$ & PSU & 2 & & & 2 & & & & 2 & & 2 & & & 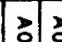 & & & & & & 25 & & $\Rightarrow$ & & & 2 & के & & & a & & & \\
\hline SPECIES & & : & & 定 & $\bar{\Sigma}$ & & 인 & & 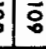 & क् & $\equiv \bar{N}$ & & $\bar{E}$ & $\bar{\omega}$ & $\bar{z}$ & $\bar{\infty}$ & $\overline{0}$ & $\overline{0}$ & $\bar{\Xi}$ & $\bar{\sim}$ & $\overline{\mathrm{s}}$ & $\overrightarrow{\tilde{N}}$ & : & & $\bar{\Xi}$ & ప్) & $\Xi$ & & $\bar{\omega}$ & & & $\bar{v}$ \\
\hline Puinqueloculina goodopeedi [?] напnа " наnna & & & & & & & & & & & & & & & & & 므 & & & & & & & & & & & & & & & \\
\hline Q. imperialis Hanna 6 Hanna & & & & & & & & & & & & & & & & & [ & & & & & & & & & & & & & & & \\
\hline Q. triangularis d'orbigny & & & & & & & & & & & & & & & {[} & & $\square$ & & $\square$ & 5 & 可 & $\square$ & & & & & & & & & & \\
\hline Q. app. & & Ot & & 0 & & & & ב] & & & & L & $\square$ & & & $\square$ & 10 & & $\square[$ & 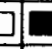 & 原 & $\square$ & $\square$ & ] & & ㅁ] & & & & & & \\
\hline Ramulina aff. R. navarroana cuahman lof mallory & & & & & & & & & & & $\square$ & & & & & & & & 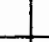 & 1 & & . & & & & 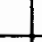 & 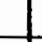 & & & & & \\
\hline Rhabdammina cooenica Cushran I G. D. Hanna & & & & & $\square$ & & $\square$ & & [] & $\square$ & O & C & & 마 & 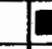 & & & & & 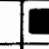 & $\square$ & & & & & & at & 1 & & & & \\
\hline R. $\{2\}$ op. & & & & & & & & & ] & & $\bar{L}$ & & $\square$ & & & & & & & & & & & & & & & & & 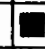 & $\square$ & \\
\hline Rabulus alatolimbatus (Gumbel) & & & & & $\square$ & & & & 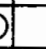 & $\square$ & OE & 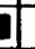 & & & & & & & & & & & & ] & & & & & & & & \\
\hline R. alatolimbatua [?] (Gumbel) & & & & & & & & & & & & & & & & & & & & & & $\square$ & & & & & & & & Z & $\square$ & I \\
\hline R. arouatootriatua var. carolinianus cushman & & & & & t & & & & $\square$ & $\square$ & $\square$ & & & & & & & & & & & & & & & & & & & & & \\
\hline R. astioulatue var. texanue (Cunhman ( Applin) & & & & & D & & & & & & & & & & & & & & & & & & & & & & & & ] & & O & \\
\hline R. artiou latus var. tezanue [?] (Cushman a Appl & & & & & & & & & & $\square$ & L & & & & & & & & & & & & & & & & & & & & 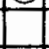 & 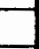 \\
\hline R. ef. R. ohehalisensia Rau [of Saith] & & $\square$ & & & & & & & & & & & & & & 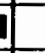 & & & & & & & & & & & & & & & & \\
\hline R. chiranus Cushman \& stone & & & & & & & & & & & & & & & & & & & & & & & & & & & & & ] & & & \\
\hline B. coaledeneie Detzing & & & & & & & & & & & & & & & & & & & & $=$ & 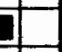 & & & & & & & & 7 & & & 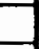 \\
\hline R. CE. $R$, ooaledenois Detling & & & & & & & & & & & & & & & & & & & & 可 & & & & & & & & & 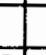 & & & \\
\hline R. inornatue (d'orbigny) & & 0 & & ] & & & a & & $\square$ & & a & & & & & & & & & & & & & & & & & & 2 & & & 口] \\
\hline R. inornatue (3] (d'orbigny) & & & & 10 & E & 可 & & & & & [ & & & [ & ] & & & & & I & & - & & & & & & & & ] & & \\
\hline R. kinoaidi Beck & & & & & & & [ & ] & $\square$ & $\square$ & & & & & ]$[\mathrm{c}$ & & & & & 단 & ] & ] & & & & & & & L & & & \\
\hline R. Cf, R. kinocidi Bock & & a & & & & & & & & & $\square$ & & & & & & & & & & & 0 & & & $\square$ & & & & 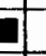 & & & \\
\hline R. Limboous var. hookleyeneie (Cuahman \& Applitn & & & & & $\square$ & & & & & $\mathbf{\square}$ & $\square$ & & & & & & & & & 도 & ] & & & ] & & & & & & & 미 & \\
\hline R. limboaue var. hookleysenais [2] (Cuahman o Ap & 1in) & & & & & & & 可 & & & & & & & $E$ & & & & [] & & & 든 & & & & & & & D] & & & \\
\hline R. Mayi Cushman \& Parker & & & & & & & & & & & & & & & & & & & & & & & & & & & & & & & & {[]} \\
\hline R. Mayi [?] Cughman \& Parker & & & & & & $\square$ & & & & $\square$ & [ & ] & & & & & & & & & & & & & & & & & ]. & & & + \\
\hline R, Cf. R, midwayenois (Plumaror) & & & & & & & & & & & & & & & [ & & & & & & & & & & & & & & & & & $\square$ \\
\hline R. propinquue oovlitagnsia Beck & & & & & & & & & & & & & & & & & & & & 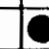 & t & & & & & & & & & & & \\
\hline $\begin{array}{l}\text { FIGUAE B. DISTARQUTION OF FOAAMINIFEAA } \\
\text { FROM THE STRATA OF CASCADE HEAD }\end{array}$ & $\begin{array}{l}\text { FAUNULLE } \\
\text { OR } \\
\text { ZONULE }\end{array}$ & 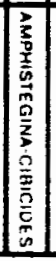 & 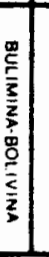 & 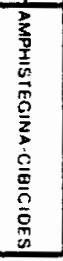 & & & & & 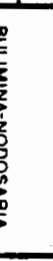 & & & & $\begin{array}{l}0 \\
0 \\
0 \\
0 \\
0 \\
0 \\
0 \\
0 \\
0 \\
0 \\
0 \\
0 \\
0 \\
0\end{array}$ & 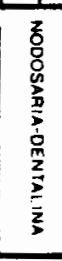 & & 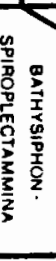 & 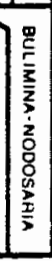 & & & 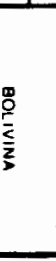 & & 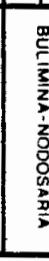 & & & 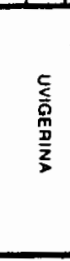 & & & & & 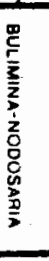 & & \\
\hline
\end{tabular}




\begin{tabular}{|c|c|c|c|c|c|c|c|c|c|c|c|c|c|c|c|c|c|c|c|c|c|c|c|c|c|c|c|c|c|c|c|}
\hline LEGEND & SECTION & & & & & & & & & & NESKO & OWIN & CAE & & & & & & & & & & & & 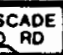 & The & & & MON & & \\
\hline $\begin{array}{lll}\text { SPECIMENS/SAMPLE } & 1-2 & 6-10 \\
\end{array}$ & PSU & 官 & & & & & & : & $\vec{a}$ & & : & & & 吾 & : & & a & 흐 & ? & & & & & & ab & & & & & & $\underline{\underline{z}}$ \\
\hline SPECIES & & $\bar{b} \mid \mathrm{s}$ & & & & & & $\overline{\mathbf{\Phi}}$ & & & $E \bar{\Sigma}$ & {$[\bar{\omega}]$} & & $\vec{a}$ & $\overline{1}$ & & & $\tilde{0}$ & $\tilde{\omega}$ & & & & & \$2 & & & & & & & \\
\hline Robulus pesudodortex cole & & & & & 미 & & & & & 미다 & $\mathrm{D}$ & & & & & & & 미 & & & & & & $\square$ & & & D] & Of & & a] & 미 \\
\hline R. Cf. R. paendovortax cole & & & & O & $\square$ & & $\square$ & & & 5 & 可 & 돈 & $\square$ & & & & & & & $\square$ & & & & & & & $\square$ & 9 & ] & E & \\
\hline R. Cf. R. terryi Coryell \& Embich & & $\square$ & & & $\square$ & & & & & & & & & & & & & & & 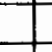 & & & & & & & 1 & 口 & & & $\square$ \\
\hline R. Weaveri Beck & & & & & & & & $\square$ & & & & & & & & & & & & & & & & & & & & & & & \\
\hline R. Weaveri $[2]$ Beck & & & & & & & $\square$ & & & 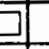 & $\square$ & & & & & & & & & & & & & & & & []] & & & & \\
\hline R. Welohi Church & & & & & & & & & & & & & & & & & & & & a & & & & 7 & & & 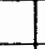 & & 田 & & \\
\hline R. spp. & & E & 9 & 0 & & $\square[$ & $\partial[$ & D & & 1 & 0 & 口] & & & $\square$ & & & {$[0]$} & 口 & ㅁ] & a & & $\square$ & 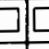 & ]$[\square$ & O & a & 0 & & oc & (1) \\
\hline Saraeenaria cf. $s$. moreatiana Howe ( Wallace & & & & & & & & & & & & & & & & & & & & & & & 6 & & & & 마 & & & & \\
\hline s. sp. & & & 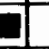 & & & & & & & & & $\square$ & & & & & & & & & $L$ & & & & & & 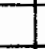 & & & 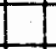 & - \\
\hline Silicosigmoilina salifornioa Curhean a Church & & & & & & & a & & & D & 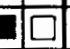 & & & & & & & $\square$ & & & $\square$ & & & & & & O) & & & & 7 \\
\hline Spiroloculina (z) of. S. lamposa Hussey & & & & & 口ा & & & $\square$ & & & & L & ( & & & & 可 & & & & 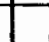 & & & & $\square$ & & & & & & ( \\
\hline S. viloozonai, Cuahman a Garrett & & & & & & & & & & & & & & & $\square$ & & & & & $\square$ & $\square$ & & [ & & & & - & & & & \\
\hline spiropleotammina diraota (Cuenman astegfue) & & & & & & & GL & 10 & (] & [] & 10 & & & & 0 & & & & & (] & $\mathrm{O}$ & ] & & o & & & & & & & \\
\hline S. tajonerais Mallory & & & & & & & & & & & & & & $\square$ & & & & & & - & & & & & & & & & & & \\
\hline s. ap. [of W11son] & & & & & & & & & & & & & & 口 & & & & & & 1 & & & 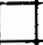 & & & $\square$ & & & & & - \\
\hline s. spp. & & $ـ$ & {[} & 可 & & & 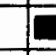 & 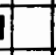 & & & 만 & & & & & & & & & 7 & & & & & & & & $\square$ & 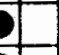 & 다 & \\
\hline Subbotina linaperta (Finlay) & & & a & & 五 & $\square$ & & & $\square$ & [ & ] & & $E$ & a & 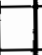 & & of & & & $\square$ & $\square$ & & & & & & O) & ofo & 4 & & 2 \\
\hline s. linaporta (3) (Finlay) & & & & & Q & ㅁ] [ & ] & & & E & 겨 & QE & 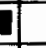 & & 미 & & & & & 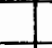 & $\square$ & & T & & & & $\square$ & & & 미 & \\
\hline s. Wileoni (Cole) & & & & & & & & $\square$ & 田 & $\mathrm{O}[\mathrm{C}$ & $\square$ & & & & & & & & & & & & $\square$ & & $\square$ & & .. & & & & \\
\hline S. vilsoni [?] (Cole) & & & & & & & {[} & & & & & & & & & & & & & 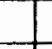 & & & - & & & & L & [ & ] & & \\
\hline Textularia [2] ap. & & & & & & & & & & & & & & & Q & & 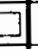 & & & & & & 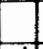 & & & & 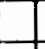 & & & & \\
\hline Trifarina advena var. oalifornioa Mallory & & & (2) & & & [] & & & & & & & & & & & & & & ㅁ. & & & D & & & & {[]} & & & & 9 \\
\hline Trilooulina trioarinata d'orblgny & & E & & & & & & & & & & & & & & & & & & & & & & & & & $\dot{.}$ & & & & \\
\hline r. [?] sp. & & $\mathbf{E}$ & & & & & & & & & & & & & & & & & & & & & & & & & - & & & & \\
\hline Iritasilina coloi Cushman a Slegfus & & 更 & & & 更 & $\square$ & $\overline{0}$ & 10 & & a & a & & & & & & & & & & & & & & & & $\square$ & & & & 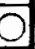 \\
\hline T. $181 \mathrm{sp}$ & & 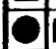 & & ] & & & & & & & & & & & & & & & & & & & & & & & & & & & \\
\hline $\begin{array}{l}\text { FIGURE 8. DISTRIBUTION OF FOAAMINIFERA } \\
\text { FROM THE STRATA OF CASCADE HEAD }\end{array}$ & $\begin{array}{c}\text { FAUNULLE } \\
\text { OR } \\
\text { ZONULE }\end{array}$ & 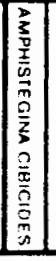 & 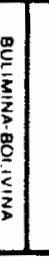 & 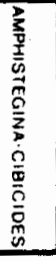 & & & & 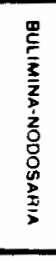 & & & & 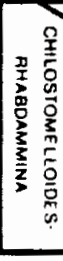 & & 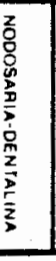 & 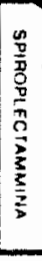 & 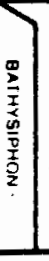 & 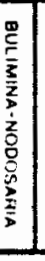 & & $\frac{\mathrm{d}}{\frac{0}{2}}$ & & & 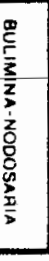 & & & & & & & 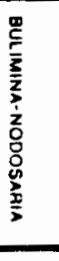 & & \\
\hline
\end{tabular}




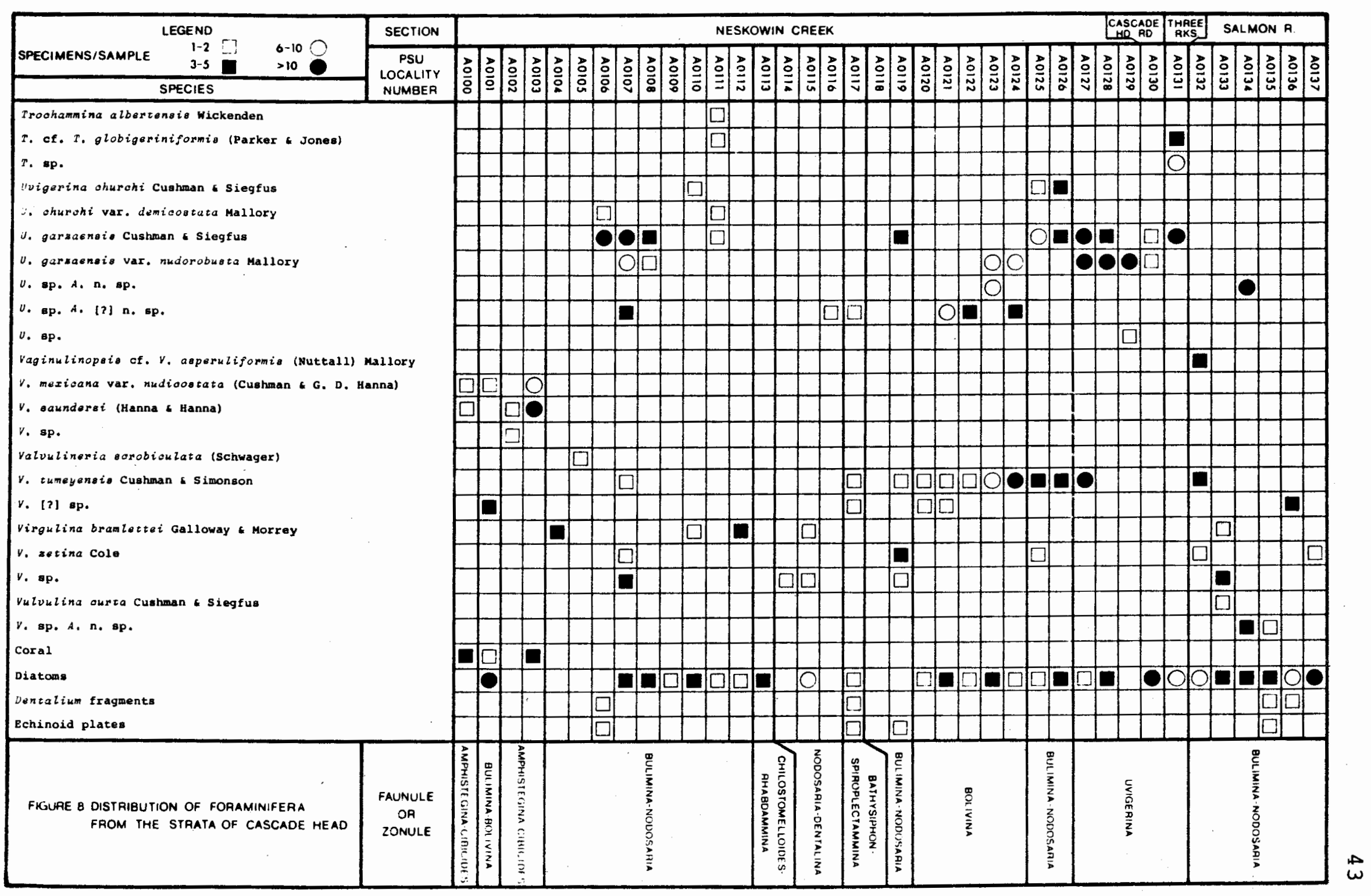




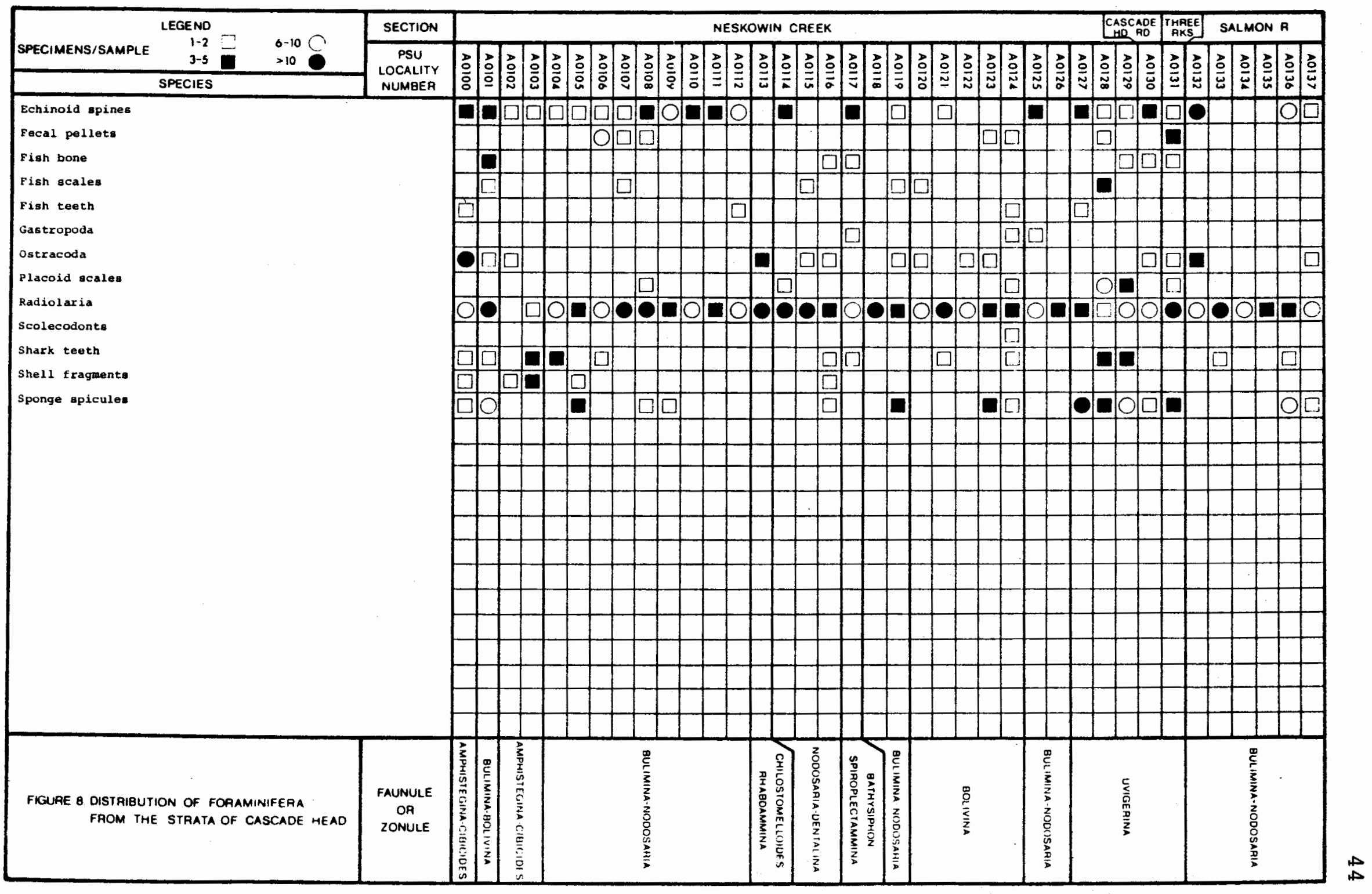




\section{BIOSTRATIGRAPHY}

\section{Local Biostratigraphy}

Eight distinct faunules (Fenton and Fenton, 1928, p. 20-22) are discernible from among the 38 foraminiferal assemblages collected from the "Strata of Cascade Head". Five of these faunules recur in at least two or more horizons within the sequence and thus, following the terminology of Fenton and Fenton (1928), are described as zonules. Measurements of these local biostratigraphic units, as well as the stratigraphic allocation of foraminiferal samples in the Neskowin Creek and Salmon River Sections, are calculated from the base of the "Strata of Cascade Head". The composition and stratigraphic occurrence of each faunule and zonule is given in the checklist (Fig. 8) and therefore will not be discussed here.

\section{Paleoecology}

The climate of the Eocene of the Cordilleran geosyncline has generally been interpreted as subtropical to tropical (Arnold, 1909, p. 532; Baldwin, 1964, p. 5). Foraminiferal faunas collected from the "Strata of Cascade Head" tend to support this interpretation in that these assemblages are composed of a rather large number of species, most of which are represented by a few individuals. Such 
varied faunas are today found in subtropical to tropical climates whereas arctic and cool temperate climates contain a few species represented by numerous individuals. The cold water temperatures ${ }^{l}$ indicated by many of the faunules and zonules in this study are probably a function of depth rather than actual surface conditions.

In this investigation, the environmental evaluation of each biostratigraphic unit is, for the most part, based on the dominant genera within each faunule, although to a lesser degree, the entire faunal assemblage has been taken into consideration. It is recognized that numerous biological, physical, and chemical factors (e.g. depth, temperature, light penetration, oceanic currents, food supply, competing organisms, nature of the substrate and salinity and oxygen content of sea water) influence the distribution of Foraminifera, but that depth and temperature are generally regarded as the most important environmental factors $(i . e$. Loeblich and Tappan, 1964; Smith, 1964; Sullivan, 1962; Hornaday, 1961). Therefore, in this study, paleoecologic interpretations are based primarily on depth and temperature. The principle references used in these interpretations are: Natland (1933), Galloway (1933), Kleinpell (1938), Mallory (1959), Bandy and Arnal (1960),

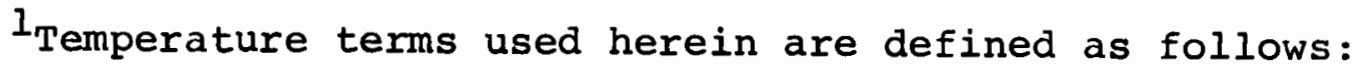
warm $20^{\circ}-16^{\circ} \mathrm{C}$ (annual minimum surface temperature) $\operatorname{cool} 16^{\circ}-10^{\circ} \mathrm{C}$ (annual minimum surface temperature) cold $10^{\circ} \mathrm{C}$ and colder (annual minimum surface temperature) 
Smith (1964) and numerous University of CaliforniaGeological Sciences publications.

The greater part of the sequence exposed in the Cascade Head area was deposited in upper to middle bathyal ${ }^{1}$ depths, although at times marine deposition fluctuated between middle neritic and bathyal. The absence of brackish water and littoral forms adds support to this interpretation. Planktonic Foraminifera, although completely absent in a few samples, occur commonly throughout the interval indicating good connections with open ocean currents. Bathymetric and temperature fluctuations (Fig. 9) are examined in greater detail in the following discussion of each individual faunule and zonule.

\section{Amphistegina-Cibicides Zonule}

The lowermost zonule, which is exposed only in the Neskowin Creek Section, is characterized by a faunule that apparently lived in warm shallow water (middle neritic) of normal marine salinity, possibly in lagoonal conditions. The most ecologically significant element included within this association is Amphistegina, which occurs in large numbers, and according to Cushman (1950, p. 300), lives

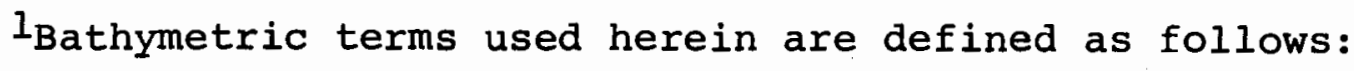

Neritic (continental shelf)

upper $\quad 0-60^{\circ}$ middle 60-300' lower 300-600'
Bathyal Tcontinental slope)

upper 600-1500' upper middle 1500-3000' lower lower 3000-6000'
Abyssal

6000-8000!

below $8000^{\prime}$ 


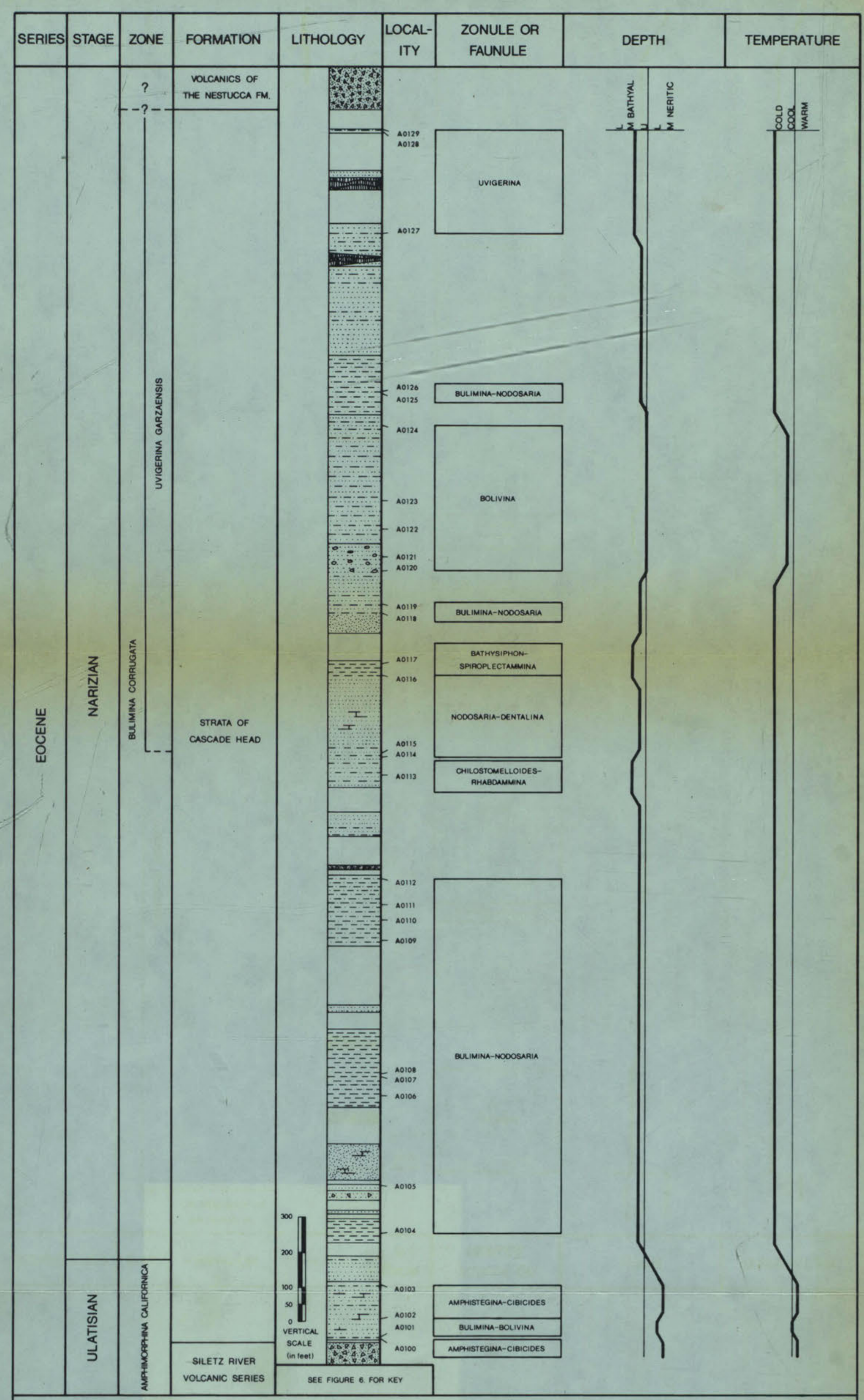

FIGURE 9. DEPTH AND TEMPERATURE VARIATION IN THE NESKOWIN CREEK SECTION 
frequently on coral reefs and in warm shallow waters. He also states that "it is probable that the genus [Amphistegina] is limited to 30 fathoms in its living condition as, like other larger foraminifera, it seems to have commensal algae, the limits of which on account of the penetration of sunlight in the ocean are limited to this same depth." Shelf conditions are also suggested by the abundance of Cibicides with thick tests (e.g. C. sandiegensis and C. memastersil. This type of Cibicides is morphologically similar to $C$. Zobatula, which has been recorded off the southern coast of California at neritic depths (Natland, 1933, chart). Similarly shallow depths are indicated by the presence of Robulus, a genus reported by Gaston (personal communication, 1976) from neritic depths. Other important calcareous forms in this zonule which favor shallow water include Elphidium, large Eponides, Quinqueloculina, Trizoculina and ornate Vaginulinopsis. Normal saline conditions are suggested by the presence of the family Miliolidae, which according to Kleinpell (1938, p. 16) cannot tolerate waters of less than normal marine salinity. The presence of many unidentifiable solitary coral and megafossil fragments also supports an interpretation of warm shallow water conditions.

The conspicuous absence of arenaceous Foraminifera in the Amphistegina-Cibicides Zonule is difficult to explain. Amphistegina faunas, completely devoid of or containing few 
arenaceous Foraminifera have also been noted by Phleger and Parker (1951, p. 61) in Recent waters of the Gulf of Mexico, by Bagley (1959, p. 50) from the lower Eocene of Washington, and by Boggs, orr, and Baldwin (1973, p. 651) from the Rickreall Limestone Member of the Yamhill Formation. Raup and Stanley (1971, p. 210) state that "Arenaceous Foraminifera today are especially common in nearshore, low salinity environments and thus are used as paleoecologic indicators." Perhaps the normal saline conditions suggested by the abundance of the Miliolids in the basal Neskowin Creek Section prohibited the development of arenaceous forms. Further ecologic investigations of recent Amphistegina faunas may provide an explanation for the absence of arenaceous Foraminifera in these assemblages. Planktonic Foraminifera are extremely rare in this zonule indicating limited open sea conditions or connections. In modern oceans, pelagic forms decrease in abundance shoreward from the outer shelf, and form a small percentage of the bottom assemblages in shallow waters and geographically restricted basins (Grimsdale and Morkhoven, 1955, in Smith, 1971, p. 13). Thus, the paucity of planktonic genera in the Amphistegina-Cibicides zonule supports an interpretation of deposition at neritic depths in possibly lagoonal conditions. 
Bulimina-Bolivina Faunule

This distinct faunule was collected from a siltstone interbed within the calcareous sandstones of the Amphistegina-Cibicides zonule, approximately 10 feet above the base of the "Strata of Cascade Head" in the Neskowin Creek section. Although many constituents of this association are essentially the same as those in the AmphisteginaCibicides Zonule (e.g. Amphistegina, Cibicides, Elphidium, large Eponides, Quinqueloculina, Robulus and Vaginulinopsis), additional forms indicate a temporary deepening (lower neritic) of the water and a continuance of warm temperatures. Other siltstone interbeds encountered within these lower calcareous sandstones, although generally unfossiliferous, probably represent similar ecologic conditions.

Bolivina, Bulimina and Cassidulina make their first appearance in this faunule and are the most abundant forms present. Bolivina, represented by both a small striate and smooth species, is probably the most ecologically important of these forms; restricted almost exclusively to neritic depths. As a group, the Buliminids are generally euryopic, although certain morphologic types have definite bathymetric preferences. Bulimina pachecoensis, a form with small marginal spines, occurs abundantly in this faunule and most closely resembles $B$. marginata which today inhabits the central and outer shelf. The presence of Bulimina corrugata, which according to Weaver and Weaver 
(1962, p. 11) prefers bathyal to abyssal optima, would seem to dispute these shallow depths but forms only a minor part of the total assemblage. Cassidulina globosa, a small rounded form whose Recent morphotype (C. subglobosa) is best developed at middle to lower neritic depths, is also suggestive of an outer shelf environment. Other significant genera occurring in lesser amounts that indicate a depth no greater than lower neritic include Cancris and Guttulina. Similarly, a depth not shallower than lower neritic is suggested by the presence of Alabamina, Chizostomelzoides, Saracenaria and Trifarina.

Although the occurrence of such forms as Amphistegina, spinose Buliminids, Quinqueloculina, Saracenaria, and Vaginulinopsis indicate warm water temperatures, the introduction of many cold water genera (e.g. Alabamina, small costae Buliminids, Cassidulina, Chilostomelloides, Nonion and Trifarinal suggests silightly cooler bottom conditions existed during deposition of the BuliminaBolivina Zonule. These cooler bottom conditions however, are probably a reflection of depth, in that warmer temperatures are found in the shallower subjacent and superjacent zonule. Arenaceous forms continue to be absent from this faunule, possibly due to the same conditions encountered in the Amphistegina-Cibicides zonule. Planktonic Foraminifera however, are abundant, signifying the existence of better connections with open ocean currents. 


\section{Buzimina-Nodosaria Zonule}

The Bulimina-Nodosaria zonule is best developed in the Neskowin Creek Section where it is repeated three times. It also characterizes the entire sequence exposed along the Salmon River. Foraminifera from this zonule are indicative of cooler and deeper water than that of the Amphistegina-Cibicides zonule in the Neskowin Creek Section. Apparently the shallow water conditions encountered in the lower Neskowin Creek sequence never existed at the base of the Salmon River Section as these sediments are completely dominated by the deeper water Bulimina-Nodosaria zonule.

A cold bathyal environment is suggested by the extremely diverse assemblage of calcareous perforate forms contained within this zonule. Shallow water forms $(e . g$. Amphistegina, Elphidium, large Eponides, and Vaginulinopsis), abundant in the underlying Neskowin Creek Section, have, for the most part, been replaced by deep water genera. Forms which normally inhabit shallow water, but have been reported from the upper limits of the continental slope (e.g. small, smooth forms of Bolivina; Caneris and Nonion), persist in reduced numbers, suggesting that depths no greater than upper bathyal existed during Bulimina-Nodosaria time. Similar conditions are indicated by the reduction in the number of shallow water Buliminids (i.e. B. pachecoensis and $B$. curtissimal and the first appearance of numerous smooth forms such as $B$. pupoides and $B$. ovata which favored 
cold bathyal depths. The Lagenids (e.g. Nodosaria, Dentalina, Marginulina, and Robulus), relatively rare in the underlying faunules, are quite diverse and occur abundantly throughout this interval. Although individually these forms are somewhat eurybathic, diverse faunas, according to Cushman (1950, p. 44) "are characteristic of the continental shelf ... between 50 and 500 fathoms ...". Further support for this interpretation of maximum depth is offered by the abundance of Nodogenerina and Ellipsonodosaria which prefer shallow depths. Cibicides with thick tests, which commonly inhabit neritic and upper bathyal depths, continue to be well represented in this zonule as do forms with thin tests (e.g. C. pachyderma and C. pseudoungerianus) that are indicative of deeper bathyal conditions. The joint occurrence of these two forms restricts the deposition of this zonule to upper bathyal depths.

Deeper water forms include the Uvigerinids, represented by both costate (e.g. U. churchi and U. sp. A.) and spinose (e.g. U. garzaensis and U. garzaensis var. nudorobusta) forms, and strongly asymmetrical species of Gyroidina (e.g. G. orbicularia var. planata and G. soldanii). Eponides umbonatus, common throughout most of the interval, has been reported from the north Pacific at bathyal to abyssal depths. The abundance of these forms indicate depths no shallower than upper bathyal existed during the deposition of this zonule. Small costate Buliminids (e.g. 
$B$. corrugata and $B$, jarvisi) reach the peak of their abundance in this zonule, also supporting an interpretation of cold temperatures at bathyal depths.

Arenaceous Foraminifera make their first appearance in this zonule, abundantly represented by Bathysiphon, spiroplectamina, and Tritaxilina. Of these, Tritaxilina is the most stenobathic, restricted almost entirely to upper bathyal depths. The other forms are somewhat eurybathic, although an abundance of Bathysiphon has generally been interpreted as representing cold bathyal depths. Lesser arenaceous elements in this association include Haplophragmoides, Karreriezla, and Rhabdammina, the presence of all of which agrees with the interpretation given above.

Pelagic forms are abundantly represented in this zonule, indicating open ocean conditions or connections. The extreme diversity of these forms suggests warm surface temperatures, in spite of the cold bottom conditions indicated by the benthonic forms.

\section{Chilostomelloides-Rhabdammina Faunule}

Continued cold bathyal depths are suggested by the rather meager fauna collected approximately 1611 feet above the base of the "Strata of Cascade Head" in the Neskowin Creek section. The sparseness of this fauna is probably a result of preservation, as numerous external molds have been observed in hand specimens. Only Chilostomezloides 
occurs abundantly in this faunule. Its Recent morphotype, Chilostomezza, has been recorded by Hornaday (1961, p. 172) as a distinctly bathyal genus. Other calcareous forms present in this faunule in lesser amounts include Globobulimina pacifica, Marginulina, and Nodosaria. Globobulimina pacifica has been reported from the lower neritic and bathyal districts of the Pacific Ocean off southern California, but finds its optimum habitat at bathyal depths. Marginulina and Nodosaria, although found at neritic depths, seem to favor the upper limits of the continental slope. AlZomorphina, which occurs only rarely in this faunule, also seems to flourish at bathyal depths. From the foregoing discussion, it would appear that this faunule lived in cold bathyal depths, probably upper or possibly middle bathyal. such an interpretation is substantiated by the arenaceous genera in this faunule, Cyclammina and Rhabdammina, both of which prefer cold bathyal depths. In addition, continued open ocean connections are suggested by the presence of numerous pelagic forms.

\section{Nodosaria-Dentalina Zonule}

Upper bathyal depths are again suggested for the next highest zonule in the Neskowin Creek Section as indicated by the persistence of many of the forms with deep water optima that were present in the underlying BuliminaNodosaria Zonule and Chilostomelloides-Rhabdammina Faunule. 
Recurring deep water calcareous forms include Globobulimina pacifica, Eponides umbonatus and strongly asymmetrical species of Gyroidina. All of these favor cold temperatures at bathyal depths. Bolivina and Nonion also reappear, suggesting depths no greater than upper bathyal. The Lagenids (e.g. Dentalina, Nodosaria, and Robulus) continue to be well diversified and, as previously noted, are characteristic of the upper limits of the continental slope. Similarly, Cibicides with both thick and thin tests reappear abundantly, indicating depths no shallower or deeper than upper bathyal. Plectofrondicularia, restricted exclusively to bathyal depths, occurs for the first time in abundance in this zonule.

Arenaceous forms, somewhat rare in this zonule, are represented by but a few individuals of Bathysiphon, Haplophragmoides, Rhabdammina, and Spiroplectammina. Although these forms can endure a great range of depths, they normally inhabit relatively cold, deep water. Planktonic Foraminifera still remain persistent, indicating continued open ocean conditions.

\section{Bathysiphon-Spiroplectammina Faunule}

Deposition in the bathyal zone (upper to possibly middle bathyal) is still indicated by this faunule, which is dominated by arenaceous forms. It was collected approximately 1931 feet above the base of the "Strata of Cascade Head" in the Neskowin Creek Section. Arenaceous 
Foraminifera, as previously noted, have generally been considered to be of little bathymetric significance, since their distribution "is governed largely by the nature of the sea bottom" (Kleinpell, 1938, p. 16). Thus, the abundance of arenaceous forms in this faunule probably indicates locally modified bottom conditions rather than any radical changes in depth. Glaessner (1948, p. 190) states that "as a rule dominantly 'arenaceous' assemblages indicate cold water conditions." This inference seems to apply to the Bathysiphon-Spiroprectammina Faunule, as numerous calcareous forms also indicate cold water conditions. Among the arenaceous forms, Spiroplectammina and Bathysiphon are the most abundantly represented, the latter indicative of cold temperatures and bathyal depths. Also present in lesser numbers are Cyclammina, Rhabdammina, and Textularia. of these, Cyclammina is the most ecologically significant, reported by Delise (1967, p. 15) as favoring bathyal depths. Calcareous perforate forms are well represented in this faunule, although not as abundantly as in the underlying intervals. The Lagenids (e.g. Dentalina, Nodosaria, and Robulus) remain diverse, suggesting upper bathyal depths. The persistence of Chilostomelzoides, Plectofrondicularia and asymmetrical species of Gyroidina, all common in the underlying unit, also support an interpretation of bathyal depths as does the reappearance of numerous smooth Buliminids. Somewhat deeper bathyal 
conditions may be indicated by the first abundant appearance of Epistomina and Pleurostomelza which, although relatively eurybathic, prefer lower bathyal and abyssal depths. Open ocean conditions are again indicated by the presence of pelagic forms.

\section{Bolivina Zonule}

Somewhat shallower depths (lower neritic to upper bathyal) than those prevailing during deposition of the preceding interval are indicated for this zonule by the recurrence of such shallow water forms as small species of Bolivina, Cancris, Cassidulina, and Nonion, all of which favor lower neritic depths. Shallower water conditions are also suggested by the increased number of Lagenids (e.g. Dentalina and Nodosaria), shallow water Cibicides (thick test), and the reappearance of ElZipsonodosaria. Associated with these shallow water genera however, are a few deeper water forms (e.g. smooth and costate Buliminids, Globobulimina, Gyroidina, Plectofrondicularia, and both spinose and costate Uvigerinids) that normally exhibit a bathyal optimum. Similarly Valvulineria (V. tumeyensis), which is more characteristic of bathyal depths, becomes significant for the first time in this zonule. Although the majority of these deeper water forms prefer bathyal depths, most can tolerate an upper slope-lower shelf environment and therefore, their presence should not be considered in conflict with the bathymetric interpretation 
given above. Also, these forms do not occur as abundantly as the shallower water genera, suggesting that they were not living within their environmental optimum.

Arenaceous Foraminifera are extremely rare in this zonule, consisting of only a few specimens of Bathysiphon and Rhabdammina. Although both of these forms favor deep bathyal depths, they are well within their environmental tolerances at upper bathyal depths. Cool bottom temperatures continue to be indicated by the presence of Cassidulina, Gyroidina, and Valvulineria, even though the depths are somewhat shallower. Planktonic Foraminifera are still well represented in this zonule, indicating continued open ocean conditions.

\section{Uvigerina Zonule}

The uppermost zonule in the Neskowin Creek Section contains a faunule which apparently lived in a deeper water environment than that of its local predecessor. It also characterizes the two supplementary sections measured near the town of Three Rocks and along Cascade Head Road. Deposition in this zonule took place at depths comparable to the upper or middle continental slope as evidenced in the diversity and abundance of genera with bathyal optima. of these deeper water forms, spinose Uvigerinids (e.g. U. garzaensis and U. garzaensis var. nudorobustal are the most abundantly represented, suggesting depths within the lower limits of the continental slope. Bathyal depths are 
also indicated by the presence of Eponides umbonatus, Epistomina, Gyroidina, Plectofrondicularia, and Valvulineria. The occurrence of Cassidulina globosa in reduced numbers in this zonule is also in keeping with an interpretation of bathyal depths and strongly substantiates cold bottom conditions. The Buliminids, abundantly represented in the subjacent faunules, are relatively rare in this zonule, although a few smooth and costate species continue to be represented. The presence of these deep water forms however, is offset by the persistence of several shallow forms (e.g. various Lagenids, Bolivina, and Cibicides with thick tests). The association of both shallow and deep water forms in this zonule suggests a middle to possibly upper bathyal environment of deposition.

Arenaceous Foraminifera are again well represented in this zonule, reflecting a change in bottom conditions. The presence of Bathysiphon and Haplophragmoides, which occur locally in considerable abundance, adds support to an interpretation of cold bathyal depths. Other important forms include Ammodiscus, Karrerielza, and Rhabdammina. of these, Karrerielza is the most stenobathic, favoring bathyal depths. Pelagic forms occur sporadically in this zonule, suggesting somewhat limited open ocean conditions locally. 
Age and Correlation

Because the teilzones of many diagnostic Californian species vary somewhat in the Northwest, it is very difficult to apply rigidly the Californian foraminiferal stages of Mallory (1959) to Oregon sections. This is due primarily to the distance separating the oregon sections from the type sections of the stages in California but also to variations in the local ecology. It also seems probable that a few species which occur within the basal sediments of the "Strata of Cascade Head" have been reworked from the underlying intervals. In addition, those species mentioned in the following discussion, whose teilzones extend into younger or older horizons in the "Strata of Cascade Head" have been excluded as important criteria from highly definitive age evaluations.

Several species, including Discorbis baintoni, Loxostomum applinae, Nodosaria latejugata, Pullenia quinqueloba, and Trifarina advena var. californica, have previously been recorded in California as ranging no higher than upper Ulatisian by Mallory $(1959$, p. 44); yet in the "Strata of Cascade Head" they occur within faunules assigned to the lower Narizian by this writer (see checklist, Fig. 8). Pleurostomelia paleocenica, which seems restricted to the upper Ynezian in California (Mallory, 1959, p. 26), also occurs within these lower Narizian assemblages. Apparently the teilzones of these species extend into younger horizons 
in the oregon lower Tertiary as they occur jointly with highly diagnostic lower Narizian forms in the "strata of Cascade Head". Similar range extensions have been recorded by authors in other lower Narizian sequences on the West Coast; both Discorbis baintoni and Nodosaria latejugata have been recorded by Gaston (1974, p. 20, 23) from the Yamhill Formation and Pleurostomella paleocenica has been recorded from the Upper Cozy Dell Shale by Weaver and Weaver (1962, p. 8). Pulzenia quinqueloba has not only been recorded from both the upper and lower Narizian (in the Cozy Dell Shale by Weaver [1962, fig. 4], the Cozy Dell Shale and the Sacate Formation by both Weaver and Weaver [1962, p. 8] and Weaver and Molander [1964, fig. 4], the Blue Canyon Gorge Member of the Twin River Formation by Carroll [1959, fig. 6], and the Boundary Shale Member of the Lyre Formation by Bagley [1959, p. 43]), but also, according to Tipton, Kleinpell, and Weaver (1973, fig. 6, 9) it apparently ranges as high as zemorrian and Saucesian in the Los Santos Shale.

Similarly, Amphimorphina jenkinsi, Anomalina regina var. minor, Bulimina sculptilis, Plectofrondicularia packardi var. packardi, and Robulus mayi, which in California range no lower than the upper Narizian (Mallory, 1959, p. 60), have extended their teilzones into lower Narizian rocks in the "strata of Cascade Head". Several of these species have also been reported by other authors from lower 
Narizian sequences on the West Coast. Amphimorphina jenkinsi, restricted entirely to the upper Narizian in California, and Robulus mayi have been reported by Weaver and Weaver (1962, p. 15) from the Matilija Formation and Upper Cozy Dell shale, respectively. Bulimina sculptilis has been recorded from the Yamhill Formation by Gaston (1974, p. 19) and Plectofrondicularia packardi var. packardi has been reported by Weaver and Molander (1964, fig. 4) from the Cozy Dell shale.

It is also important to note that, although major biostratigraphic units (stages and zones) are easily recognized in the "Strata of Cascade Head", the boundaries between these units are often difficult to delineate precisely. This is due to the absence of certain diagnostic forms at both the upper and lower boundaries of these units. Samples collected near the base of the Bulimina corrugata zone in the Neskowin Creek Section, for example, contain species that have their last known occurrence in this zone but completely lack forms that have their first known occurrence, whereas samples collected near the top of the zone contain species that have their first known occurrence but lack forms that have their last known occurrence in this zone. Thus, only that portion of this interval, where both first and last appearing forms occur jointly, can be definitely assigned to the Bulimina corrugata Zone. The lack of either first known occurrences at the base of a zone 
or last known occurrences at the top of a zone permit only an approximate boundary to be drawn between two biostratigraphic units.

on the basis of its contained foraminifers, the lower portion of the Neskowin Creek Section can be assigned to the upper Ulatisian Stage of Mallory (1958, p. 43-44), whereas the balance of the "strata of Cascade Head" can be correlated with Mallory's lower Narizian Stage (1959, p. 57-60). Of the 3495 foot section measured along Neskowin Creek, the lower 168 feet is assigned to the Amphimorphina californica Zone (upper Ulatisian) and the upper 3178 feet is assigned to the Bulimina corrugata Zone (lower Narizian). The Amphimorphina californica-Bulimina corrugata boundary, lying somewhere within the 149 foot interval between these two units, cannot be delineated accurately because of the lack of certain diagnostic forms at the base and top of these units and the lack of a continuously fossiliferous sequence.

Although poorly preserved, Foraminifera from localities in the lowermost 168 feet of the section measured along Neskowin Creek are representative of Mallory's Amphimorphina californica zone. This is indicated by the joint occurrence of Bolivina huneri, which ranges no higher than the upper Ulatisian, and Gyroidina orbicularis var. planata, which ranges no lower than the upper Ulatisian. Perhaps more importantly however, is the presence of Amphistegina 
simiensis in this interval. This species, originally described by Cushman and McMasters from the middle Eocene Llajas Formation in California, has never been recorded from rocks younger than middle Eocene on the West coast and seems to be somewhat restricted to this interval (Bagley, 1959, p. 31, Bandy, 1944, p. 366; and Fairchild, Wesendunk, and Weaver, 1969, p. 16). Regarding the range of the genus Amphistegina in the Northwest, Stewart (in Baldwin, 1964b, p. 12) states:

All published references to Amphistegina and Discocyclina in Oregon and Washington that have come into hand have assigned a middle Eocene age to the sediments in which these genera were found.

He further states on p. 13:

The files of the Oregon Department of Geology and Mineral Industries contain no record of the occurrence of Discocyclinas or Amphisteginas in oregon rocks known to be younger than middle Eocene age, although it is not uncommon to find them in rocks of known middle Eocene age.

Also present in this interval is Vaginulinopsis mexicana var. nudicostata, a species reported by Mallory (1959, table 19) in California as ranging no higher than the Amphimorphina californica zone. Additional forms which are common in, but not confined to, the upper Ulatisian include Eponides mexicana, Cibicides sandiegensis and Cibicidoides coalingensis.

The upper 3178 feet of the Neskowin Creek Section contains an extremely diverse foraminiferal congregation that can be best correlated with the Bulimina corrugata 
Zone of Mallory. This is indicated by the presence of the

following species known from horizons no older than lower

Narizian:

Bolivina basisenta

Bulimina microcostata

Cibicides laurisae

Haplophragmoides latissimisuturalis (see Mallory, 1959, table 19, as $H$. obliquicameratus)

Nodogenerina cf. N. bradyi (see Mallory, 1959, table 19, as $N$. bradyi)

Nodosaria chirana

Planularia markleyana

Planularia cf. P. ouachitaensis

Plectofrondicularia packardi var. multilineata

Plectofrondicularia searsi

Plectofrondicularia vokesi

Pullenia salisburyi

Quinqueloculina imperialis

Uvigerina churchi

Uvigerina churchi var. demicostata

Uvigerina garzaensis

Uvigerina garzaensis var. nudorobusta

Valvulineria tumeyensis

Virgulina bramlettei

and from horizons no younger than lower Narizian:

Alabamina wilcoxensis var. californica

Anomalina crassisepta

Anomalina dorri var. aragonensis

Bulimina ourtissima

Cibicides laimingi

Dentalina angusticostata

Dentalina hexacostata

Dentalina Cf. D. sherborni

Globigerina decepta

Gyroidina soldanii var. octocamerata

Karrerielza chapapotensis var. monumentensis

Marginulina eximia

Nodosaria deliciae

Nodosaria (?) gyrata

Nonion durhami

plectofrondicularia kerni

Pseudohastigerina micra

Ramulina aff. $R$. navarroana

Robulus pseudovortex

Valvulineria scrobiculata

Additional evidence for this age determination is offered 
by the following species: Alzomorphina macrostoma, which Smith (1971, p. 21) reports as confined to the Bulimina corrugata zone in the Santa Cruz Mountains of California; Bulimina consanguinea and Karrerielza elongata, perhaps restricted to lower Narizian horizons (see Mallory, 1959, table 19) and Cibicides pachyderma and Robulus cf. $R$. terryi, both of which according to Mallory (1959, table 19) appear to range no higher than the lower Narizian. Species occurring abundantly in this interval that are characteristic of, but not confined to, the Bulimina corrugata Zone include Bathysiphon eocenicus, Bulimina corrugata, Cibicides felix, Cibicidoides venezuelanus, Gyroidina orbicularis var. planata, Robulus altolimbatus, spiroplectamina directa, and Tritaxilina colei.

More particularly, the uppermost 1504 feet of the Neskowin Creek Section, between samples PSUA0115 and PSUA0129, can be assigned to the Uvigerina garzaensis Subzone of Mallory (1959, p. 60-61) as Bolivina basisenta, Cibicides Laurisae, Planularia markleyana, Plectofrondicularia searsi, and Plectofrondicularia vokesi have been reported no lower than this subzone and Nodosaria (2) gyrata has been reported no higher. Other species characteristic of this subzone which occur within this interval include Rhabdammina eocenica, spiroplectammina directa, and Tritaxilina colei. To the author's knowledge, this is the first record of the Uvigerina garzaensis subzone in rocks 
north of California. It should also be noted that although the upper subzone can be recognized in the Neskowin Creek Section, the presence of the lower or Uvigerina churchi Subzone cannot be demonstrated in this sequence because of the absence of the chronologic indicators which characterize the unit.

The three supplementary sections measured in the Cascade Head area are also assigned to the Bulimina corrugata zone of Mallory. In the Cascade Head Road and Three Rocks sections this is indicated by the presence of Haplophragmoides Latissimisuturalis (see Mallory, 1959, table 19, as $H$. obliquicameratus), Nodogenerina cf. $N$. bradyi (see Mallory, 1959, table 19, as N. bradyi), Plectofrondicularia packardi var. multilineata, Plectofrondicularia searsi, Pullenia salisburyi, Uvigerina garzaensis, and Uvigerina garzaensis var. nudorobusta and to a lesser degree by the stratigraphic equivalence of these intervals with localities at the top of the Neskowin Creek Section. Also occurring within these intervals are Bathysiphon eocenicus and Gyroidina orbicularis var. planata, both of which are characteristic of lower Narizian horizons. Moreover, since both the Cascade Head Road and Three Rocks Sections occupy the same stratigraphic position as the upper Neskowin Creek Section, the Uvigerina garzaensis Subzone is probably represented in these sequences. However, because of the lack of chronologic indicators, these 
sequences have not been assigned to this subzone. The Salmon River Section can be correlated with the Bulimina corrugata zone of Mallory on the basis of the joint occurrence of the following species which are not known to occur above this zone:

Alabamina wilcoxensis var. californica Bulimina curtissima Clavulinoides californicus

Dentalina hexacostata Gyroidina soldanii var. octocamerata Marginulina eximia Pseudohastigerina micra Robulus pseudovortex

and species which are not known to occur below this zone:

Cibicides Zaurisae

Valvulineria tumeyensis

Virgulina bramlettei

Karreriella elongata, a species restricted to the lower Narizian Stage (Mallory, 1959, table 19), also appears in this interval. Other forms collected from this section which apparently range no higher than the Bulimina corrugata Zone (Mallory, 1959, table 19) include Cibicides pachyderma, Cibicides spiropunctatus, Robulus cf. R. terryi, and Pseudoglandulina conica. Also common in this interval are Bathysiphon eocenicus, Bulimina corrugata, and Gyroidina orbicularis var. planata, all of which characterize lower Narizian faunas. Finally, conflicting evidence is presented by the occurrence at the base of the Salmon River section of single specimens of Amphistegina simiensis and Marginulina huneri, species reported by Mallory (1959, p. 94 and table 19) as occurring no higher than the upper 
Ulatisian Stage. However, since these Ulatisian species, indicative of shallow water, occur with a highly diagnostic lower Narizian deep water assemblage, it seems probable that they have been reworked from the subjacent sedimentary interbeds of the Siletz River Volcanic Series, which characteristically contain a shallow water Ulatisian fauna (stewart in Baldwin, 1964b, p. 12-13).

Although its lower limits have been clearly established in the Neskowin Creek Section, the upper boundary of the Bulimina corrugata Zone cannot be placed within the "Strata of Cascade Head". The highest samples in the Neskowin Creek, Cascade Head Road, and Three Rocks Sections contain faunas assigned to the Bulimina corrugata zone. Thus, the boundary between the Bulimina corrugata and overlying Amphimorphina jenkinsi zone must fall somewhere within the superjacent Nestucca Formation as foraminiferal samples collected by snavely and Vokes (1949) to the north and south of the Cascade Head area indicate that the Nestucca Formation is, for the most part, referable to the upper Narizian Stage of Mallory. The presence of Plectofrondicularia packardi var. packardi and Amphimorphina jenkinsi, species normally indicative of the upper Narizian stage, near the top of the Neskowin Creek section, suggests that this boundary probably lies somewhere close to the base of the superjacent unit. 
Several West Coast Formations have yielded foraminiferal faunas that can be correlated with those of the "Strata of Cascade Head", either in part or as a whole. of these, the Rickreall Limestone Member of the Yamhill Formation, as described by stokesbary (1933, in Baldwin, $1964 \mathrm{~b}, \mathrm{p} .21$ ) from near the town of Dallas, Oregon, bears the closest faunal affinity to the upper Ulatisian portion of the "Strata of Cascade Head". Foraminiferal faunas from other basal calcareous lenses in the Yamhill Formation (see various authors in Baldwin, 1964b, p. 21-24, and Boggs, Orr, and Baldwin, 1973) are also strikingly similar to the Ulatisian faunas of the "Strata of Cascade Head". In California a similar assemblage has been reported from the upper Llajas Formation (upper Ulatisian) by Cushman and McMasters (1936). Other formations on the West coast assigned to the upper Ulatisian which are at least in part equivalent to the lowermost "Strata of Cascade Head" include the Eocene beds at Cape Blanco (Bandy, 1944) and the Elkton Siltstone, Lorane Siltstone, and Sacchi Beach Members of the Tyee Formation of southwestern Oregon; as well as the McIntosh Formation and possibly the Crescent Formation of southwestern Washington. Californian equivalents include the Canoas Siltstone Member of the Kreyenhagen Formation, the Domengine Sandstone, the middle portion of the Matilija Formation, the upper part of the Muir Sandstone, the lower part of the Nortonville Shale, 
and the upper Vacaville Shale.

It is interesting to note that in the coast Range of oregon and Washington, calcareous sediments are exposed at the base of most late middle to late Eocene age sequences which directly overlie lower Tertiary submarine basalts. To the north along the Olympic Peninsula, rocks of equivalent age comprising the Boundary Shale Member of the Lyre Formation (Bagley, 1959), the Aldwell Formation (Thoms, personal communication, 1976), and the "Maynard" Sandstone of Thoms (1959) and Allison (1959) in the Port Townsend area all possess basal calcareous strata. Similarly, calcareous sediments are exposed, as previously mentioned, at the base of the Yamhill Formation (Baldwin, 1964b) and the "Strata of Cascade Head". Foraminiferal assemblages from these calcareous units normally contain very similar constituents and are, for the most part, correlative. Baldwin (1964b, p. 21) attributes the basal calcareous strata of the Yamhill Formation to weathering of calcite veinlets and interstices within the Siletz River volcanic Series, which formed a highland at the time. Calcareous sediments accumulated as lime-rich streams flowed into localized basins or lagoons where warm sea water and marine organisms aided deposition. Information gathered during this investigation is entirely compatable with Baldwin's interpretation as the basal "Strata of Cascade Head" contain a very warm shallow water fauna (middle neritic) 
and numerous basaltic fragments presumably derived from weathering of the Siletz River Volcanic Series.

The extremely diverse foraminiferal assemblages

collected from the lower Narizian portion of the "strata of Cascade Head" permit correlation with many West coast Formations. In oregon and Washington, formations which are at least in part equivalent include the Aldwell Formation, the Coaledo? Formation of Baldwin (1961; to be formalized under the term Bateman Formation), the Boundary Shale Member of the Lyre Formation, the "Maynard Formation" of Thoms (1959) and Allison (1959), the McIntosh Formation, the Moody Shale Member of the Toledo Formation, and the Yamhill Formation. In California, lower Narizian equivalents include the Butano Formation, Cozy Dell Shale, Escobar Sandstone, lower Kreyenhagen Formation, lower Markley Sandstone, upper Matilija Sandstone, and the Poway Conglomerate.

Geologic History

A model reconstructing the middle Tertiary history of the "Strata of Cascade Head" can be developed as a result of the detailed information gained during this investigation. According to Snavely, Wagner, and Macleod (1969, p. 36) western Oregon and Washington was the site of a large subsiding trough throughout most of the early Tertiary, which extended from Vancouver Island southward to the Klamath Mountains. During much of the early and 
middle Eocene, vast submarine outpourings of basaltic lava (the Siletz River Volcanic Series in the Central oregon Coast Range) accumulated on the floor of this rapidly subsiding basin. Toward the close of the volcanic period (lower Ulatisian), extrusion of lava exceeded the subsidence of the basin and in many places volcanic islands were formed. At the margins of these islands, calcareous sediments, derived from the weathering of the volcanics, were deposited in restricted embayments or lagoons (Baldwin, 1964b, p. 21). The lower calcareous sandstones of the Neskowin Creek Section were probably deposited in such an environment as upper Ulatisian faunas are indicative of warm shallow waters (middle neritic). Similarly, the basaltic fragments included within these deposits were apparently derived from the Siletz River Volcanic Series. Marine deposition fluctuated between middle and lower neritic depths during this time as a few siltstone interbeds within the calcareous sandstones contain lower neritic assemblages. The lack of upper Ulatisian strata at the base of the Salmon River Section, exposed less than four miles to the east, suggests that this area remained a topographic high during upper Ulatisian times since underlying and overlying rocks appear to be lithologically equivalent in both sections.

As early Narizian time began, the volcanic highs in the Cascade Head area were buried by arkosic sand and mud 
during continued submergence of the basin. This is evident in the thick sequences of mudstone and thinly laminated beds of micaceous arkosic sandstone observed above the Siletz River Volcanic Series in the Salmon River section and the lower calcareous sandstone in the Neskowin Creek section. Foraminiferal assemblages from these sequences are essentially equivalent and indicate deposition took place at depths comparable to the upper continental slope (upper bathyal). Just prior to Uvigerina garzaensis Subzone time, a profound change occurred within the cascade Head area as the sediments become dominated by volcanic material. This is evidenced by the basaltic debris and tuffaceous nature of the rocks exposed along Neskowin Creek. Flows of basaltic lava and breccia also occur sporadically, intercalated with the clastic sediments. Foraminiferal faunas indicate deposition took place primarily at upper bathyal depths. Shallower depths, as evidenced by coarsegrained sediments, probably correspond with increased periods of volcanism. Near the top of the sequence depths become progressively greater (middle bathyal) prior to the extrusion of the superjacent volcanics of the Nestucca Formation. 
SYSTEMATIC CATALOGUE

In the following synonymies, all identifications are based primarily on published figures and descriptions. Special note is made herein of species that have been compared with type material on deposit at the University of California-Berkeley and Portland State University. No attempt has been made to develop a complete synonymy for each species and only the publications actually used in the identification are included. Nevertheless, the catalogue is reasonably complete in regard to publications on the West coast Tertiary.

All of the type material is deposited in the micropaleontological collections of the Earth Sciences Museum at Portland State University. All type and locality numbers refer to the collections at Portland State University. The systematic arrangement of the Foraminifera follows Cushman (1950) except in the case of planktonic Foraminifera, in which the classification of Loeblich and Tappan (1964) is used.

PhYlum PROTOZOA

Class SARCODINA Butschli, 1882

Order FORAMINIFERA d'Orbigny, 1826

Family ASTRORHIZIDAE 
Bathysiphon sp.

Hypotype no. 00196, loc. PSUA0131.

Several individuals from the Three Rocks Section differ from B. eocenicus (Mallory, 1959, pl. 1, fig. 4) in possessing a more coarsely arenaceous test.

\section{Family HYPERAMMINIDAE}

Genus Hyperammina H. B. Brady, 1878

Hyperamina [?] sp.

Hypotype no. 00197, loc. PSUA0100.

Fragmented specimens from the Neskowin Creek Section resemble Mallory's figure of $H$. elongata H. B. Brady (?) (1959, pl. 1, fig. 8, pl. 27, fig. 2) but lack the bulbous proloculus and tapering second chamber that characterize this genus (Loeblich \& Tappan, 1964, p. C190; Smith, 1971, p. 24) .

\section{Family AMMODISCIDAE}

Genus Ammodiscus Reuss, 1861

Ammodiscus incertus (d'orbigny)

Ammodiscus incertus (d'orbigny), Howe, 1939, p. 29, pl. 1,

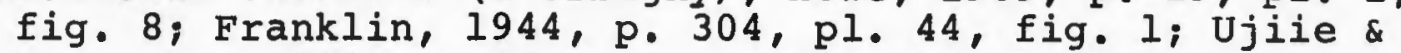
Watanabe, 1960, p. 127, pl. 1, fig. 9a, b; Weaver \& Molander, 1964, p. 177, pl. 1, fig. 6.

Hypotype no. 00198, loc. PSUA0111

Ammodiscus cf. A. incertus (d'orbigny) [of Mallory]

Ammodiscus cf. A. incertus (d'Orbigny), Mallory, 1959, p. 108, pl. 1, fig. 11, 12, pl. 39, fig. 2; Kleinpell \& Weaver, 1963, p. 166, pl. 2, fig. 6; Weaver \& Molander, 1964, p. 177, pl. 1, fig. 7 .

Hypotype no. 00199, loc. PSUA0131. 
Comparison of this species with the hypotypes of $A$. incertus suggest that the compressed shape of $A$. cf. $A$. incertus may have resulted from distortion during preservation. Further studies should prove the two to be synonymous. Ammodiscus sp.

Hypotype no. 00200, 10c. PSUA0129.

A few incomplete individuals are referable to this genus. Family LITUOLIDAE

Genus Haplophragmoides Cushman, 1910

Haplophragmoides Latissimisuturalis Smith

Hap Zophragmoides obliquicameratus Marks, 1951, p. 35, pl. 5, fig. la-c; Sullivan, 1962, p. 251, pl. 9, fig. 9a, b, $10 a, b$; Weaver, 1962, p. 369, pl. 1, fig. 4; Kleinpell \& Weaver, 1963, p. 166, pl. 2, fig. 7; Fairchild, Wesendunk, \& Weaver, 1969, p. 30, pl. 1, fig. 6a, b, 7a, b; Gaston, 1974, p. 46 , [hypotype examined].

Haplophragmoides sp. Wilson, 1954, p. 131, pl. 12, fig. 4 a, b. Haplophragmoides sp. Smith, 1956, p. 87, pl. 9, fig. 3a, b. Haplophragmoides cf. H. obliquicameratus Marks, Hornaday, 1961, p. 179, pl. 1, fig. 8a-c; Weaver \& Molander, 1964, p. 178, pl. 1, fig. 12, 13 .

Haplophragmoides Latissimisuturalis Smith, 1971, p. 25, pl. 1, fig. 6a-c, pl. 2, fig. la, b.

Hypotype no. $00201,10 c$. PSUA0130.

Haplophragmoides obliquicameratus was described by Marks (1951, p. 35) in his revision of a number of species from the Vienna Basin. Subsequently, Smith (1971, p. 26) observed differences between $H$. obliquicameratus and forms previously referred to this species in West Coast Tertiary deposits and redescribed it as $H$. Latissimisuturalis. In her 
description she states:

The confusion with $H$. obliquicameratus results from mistaking chambers for sutures [chambers are commonly depressed and sutures raised upon fossilization]. Marks' description and figures (1951, p. 35, pl. 5, fig. la-c) depict a form with nonlimbate, depressed sutures. H. obliquicameratus also has about eleven chambers in the final whorl, compared to the six to eight of the California specimens.

Individuals from the "Strata of Cascade Head" are typical of the West Coast forms in that they average eight chambers in the final whorl and have limbate sutures.

$$
\text { Haplophragmoides sp. }
$$

Hypotype no. 00202, loc. PSUA0111.

Poorly preserved individuals seem best placed in this genus.

$$
\begin{aligned}
& \text { Genus Cribrostomoides Cushman, } 1910 \\
& \text { Cribrostomoides [?] sp. }
\end{aligned}
$$

Hypotype no. 00203, loc. PSUA0105.

Because its apertural characteristics cannot clearly be distinguished, a single specimen from locality PSUA0105 is questionably placed within this genus.

Genus Cyclammina H. B. Brady, 1876

Cyclammina clarki (G. D. Hanna)

? Nonionina clarki Hanna, 1923, p. 24, pl. 59, fig. 2a, b. Cyclammina clarki ? (Hanna), Cushman \& Schenck, 1928, p. 306, pl. 42, fig. 1; Weaver \& Molander, 1964, p. 178, pl. 2, fig. $2 a, b$.

Cyclammina cf. C. Clarki (Hanna), Wilson, 1954, p. 132, pl. 12, fig. 5a, b; Smith, 1956, p. 87, pl. 9, fig. 5a, b; Hornaday, 1961, p. $180, \mathrm{pl}$. 1, fig. 9 a-c. 
Cyclammina clarki (Hanna), Mallory, 1959, p. 114, pl. 27, fig. 4a, b, pl. 39, fig. 4a, b; Sullivan, 1962, p. 251, pl. 2, fig. 3; Weaver \& Weaver, 1962, p. 19, pl. 1, fig. 11, 12; Kleinpel1 \& Weaver, 1963, p. 167, pl. 2, fig. 9; Gaston, 1974, p. 47, [hypotype examined].

Hypotype no. 00204, loc. PSUA0131.

Most published figures that have appeared in West Coast literature which refer to C. clarki, C. clarki?, or C. cf. C. clarki can be divided into two groups: a group which is ovoid in plan view (Cushman and Schenck, 1928; Hornaday, 1961; Sullivan, 1962; Weaver and Weaver, 1962; Kleinpell and Weaver, 1963; and Weaver and Molander, 1964) and a group which is circular in plan view (Hanna, 1923; Wilson, 1954; Smith, 1956; and Mallory, 1959). Rather than regarding each group as a separate species, it is suggested that they be synonymized (the ovoid group representing distorted members of the circular group), since the only apparent difference between these forms is the shape of the test. It is interesting to note that in samples in which distorted specimens of $C$. clarki occur (PSUA0115, PSUA0117, and PSUA0131), other forms that appear to be distorted are also present (Haplophragmoides Zatissimisuturalis and Ammodiscus cf. A. incertus).

C. pacifica Beck is very difficult to differentiate from C. clarki. Both possess broadly rounded peripheries, slightly incised sutures, and average ten chambers in the final whorl (Hanna, 1923, p. 323; Beck, 1943, p. 591). Smith (1971, p. 30) has even synonymized the two forms. 
Until further comparison of the primary type is accomplished these forms will remain separated in this investigation. Cyclammina incisa (stache)

[?] Cyclammina incisa (Stache), Cushman, Stewart, \& Stewart, $1947 \mathrm{a}, \mathrm{p} .12$, pl. 1, fig. la, b.

Cyclammina cf. C. incisa Stache, Smith, 1956, p. 88, pl. 9, fig. $4 a, b$; Sullivan, 1962, p. 252, pl. 2, fig. la, b.

Cyclammina incisa (Stache), Asano, 1958, pl. 13, fig. 20a, b; Kleinpell \& Weaver, 1963, p. 167, pl. 2, fig. 1la, b; Smith, 1971, p. 30, pl. 2, fig. 3a, b; Tipton, Kleinpell, \& Weaver, 1973, p. 4l, pl. 1, fig. 2a, b.

Hypotype no. 00205, loc. PSUA0117.

C. incis $a$ is differentiated from other members of its genus by slightly curved sutures, an acute somewhat lobate periphery, and a thin compressed test.

$$
\text { Cyclammina incisa [?] (Stache) }
$$

Hypotype no. 00206 , loc. PSUA0116

Cyclammina samanica Berry

Cyclammina cf. C. samanica Berry, Cushman \& Simonson, 1944 , p. 193, pl. 30, fig. 2; Wilson, 1954, p. 132, pl. 12, fig. $5 a, b$.

Cyclammina samanica Berry, Mallory, 1959, p. 115, pl. 2,

fig. 15a, b; Hornaday, 1961, p. 180, pl. 1, fig. 10a-c;

Sullivan, 1962, p. 252, pl.2, fig. 2; Weaver \& Weaver, p. 19, pl. 1, fig. 13; Kleinpell \& Weaver, 1963, p. 167, pl. 3, fig. 2; Weaver \& Molander, 1964, p. 187, pl. 2, fig. $3 a, b, 4 a, b$; Delise, 1967, p. 23, pl. 1, fig. 2a, b; Fairchild, Wesendunk, \& Weaver, 1969, p. 31, pl. 1 , fig. $9 a, b$; Gaston, 1974, p. 48, [hypotype examined]. Hypotype no. 00207, loc. PSUA0137.

Typical specimens average eight chambers in the last whorl and are extremely compressed in lateral view. 
Cycrammina sp.

Hypotype no. 00208, loc. PSUA0117.

Family TEXTULARIIDAE

Genus Spiroplectammina Cushman, 1927

Spiroplectammina directa (Cushman \& Siegfus)

Spiroplectoides directa Cushman \& Siegfus, 1939, p. 26, pl. 6, fig. 7, 8.

Spiroplectammina directa (Cushman \& Siegfus), Mallory, 1959,

p. 116, pl. 3, fig. 5a, b; Fairchild, Wesendunk, \& Weaver, 1969, p. 31, pl. 1, fig. 10; Gaston, 1974, p. 48, [hypotype examined].

Spiroplectammina cf. S. directa (Cushman \& Siegfus), Fairchild, Wesendunk, \& Weaver, 1969, p. 31, pl. 1, fig. 11.

Hypotype no. 00209 (microspheric individual), loc. PSUA0125. Hypotype no. 00210 (megalospheric individual), lOC. PSUA0125.

\section{Spiroplectammina tejonensis Mallory}

Spiroplectammina tejonensis Mallory, 1959, p. 118, pl. 3, fig. $6 a, b, 7 a, b, 8 a, b$.

[Non] Spiroplectammina tejonensis Mallory, Tipton, Kleinpell, \& Weaver, 1973, p. 4l, pl. 1, fig. $4 \mathrm{a}, \mathrm{b}$.

Hypotype no. 00211 , loc. PSUA0116.

$$
\text { Spiroplectammina sp. [of Wilson] }
$$

Textularia ? sp. Cushman \& Simonson, 1944, p. 193, pl. 30. fig. 3.

Spiroplectammina sp. Wilson, 1954, p. 132, pl. 12, fig. 7a, b. Spiroplectammina sp. Hornaday, 1961, p. 181, pl. 2, fig. la, b.

Textularia cf. T. warreni Cushman \& Ellisor, Sullivan, 1962, p. 253, pl. 2, fig. 8a, b, 9 .

Spiroplectammina mississippiensis (Cushman), Kleinpell \& Weaver, 1963, p. 167, pl. 3, fig. 7a, b; Gaston, 1974, p. 49, [hypotype examined]. 
Hypotype no. 00212 (microspheric individual), loc. PSUA0131. Hypotype no. 00213 (megalospheric individual), IOC. PSUA0116

Specimens collected from the Neskowin Creek and Three Rocks Sections are conspecific with $S$. sp. of Wilson (1954, pl. 12, fig. 7a, b). Placement of these specimens within the genus spiroplectammina results from the presence of a planispiral coil in the early portion of the tests. The planispiral coil consists of four chambers followed by ten biserially arranged chambers in megalospheric individuals and 18 biserially arranged chambers in microspheric individuals. This distinctive species is characterized by slightly curved, limbate sutures and a very compressed test; the center and margins of the test are thicker than the areas between them.

Further study of comparative material will probably warrant the proposal of a new name.

spiroplectammina spp.

Genus Textularia Defrance, 1824

TextuZaria [?] sp.

Hypotype no. 00214, loc. PSUA0117.

Fragmented and poorly preserved individuals are questionably assigned to this genus.

$$
\begin{aligned}
& \text { Genus Vulvulina d'orbigny, } 1826 \\
& \text { Vulvulina curta Cushman \& Siegfus }
\end{aligned}
$$

Vulvulina curta Cushman \& Siegfus, 1935, p. 91, pl. 14, fig. 1, 2; Mallory, 1959, p. 119, pl. 3, fig. 12a, b, $13 a, b$, pl. 27, fig. 6a, b; Fairchild, Wesendunk, \& Weaver, 1969, p. 32, pl. 2, fig. 3a, b; Gaston, 1974, p. 49, [hypotype examined]. 
Vulvulina cf. V. curta Cushman \& Siegfus, Weaver \& Weaver, 1962 , p. 20 , pl. 2 , fig. 3 .

Hypotype no. 00215, 10c. PSUA0133.

Vulvulina sp. A. [species nova]

(P1. 1, fig. 1, 2)

Test comparatively large, much compressed, longer than broad, broadest at apertural end, curved after biserial stage, periphery slightly rounded and spinose; chambers distinct, earliest arranged in planispiral coil, four in number, followed by six to eight biserial chambers, later chambers uniserial, nine in number; sutures distinct, curved, depressed; wall finely arenaceous; aperture in adult, terminal, elongate

Dimensions of hypotypes:

00216 ; length $1.10 \mathrm{~mm}$.; width .48 m.

00217; length .79 mm.; width .48 m.

Hypotype no. $00216,10 \mathrm{c}$. PSUA0134.

Hypotype no. 00217, loc. PSUA0134.

This species most closely resembles Vulvulina pennatula (Batsch) var. italica Cushman (1932b, p. 78, pl. 10, fig. 6) but differs in the curvature of the test after the biserial stage and the more numerous chambers in the uniserial stage.

Specimens commonly break between the biserial and uniserial stages resulting in a biserial fragment that is easily confused with species of the genus spiroplectammina. However, inspection of the apertural region should allow placement in the correct genus; Spiroplectammina has an aperture at the inner margin of the last chamber while vulvulina has an elongate slit on the final chamber. The naming of this species must await publication in accordance with the International Code of zoological Nomenclature (1961, p. 9, art. $11 a)$ 
Family VERNEUILINIDAE

Genus Gaudryina d'Orbigny, 1839

Gaudryina cf. G. Zaevigata Franke [of Mallory]

Gaudryina (Gaudryina) Zaevigata Franke, Smith, 1957, p. 152, pl. 18, fig. 8a, b.

Gaudryina cf. G. Laevigata Franke, Mallory, 1959, p. 122 , pl. 4, fig. 3a, b.

Hypotype no. 00218, 10c. PSUA0137.

$$
\text { Gaudryina [?] sp. }
$$

Hypotype no. 00219, 10c. PSUA0108.

A single individual from the Neskowin Creek Section may represent this genus.

$$
\begin{aligned}
& \text { Genus Clavulinoides Cushman, } 1936 \\
& \text { Clavulinoides californicus Mallory }
\end{aligned}
$$

Gaudryina (Pseudogaudryina) sp. Smith, 1957, p. 153, pl. 18, fig. $13 a-c, 14 a-c$.

Clavulinoides californicus Mallory, 1959, p. 123, pl. 4, fig. $6 a, b$.

Hypotype no. 00220 , loc. PSUA0137.

\section{Family VALVULINIDAE}

$$
\text { Genus Dorothia Plummer, } 1931
$$

Dorothia principiensis Cushman \& Bermudez

Dorothia principiensis Cushman \& Bermudez, Cushman \& Siegfus, 1939, p. 24, pl. 6, fig. 23; Bergquist, 1942, p. 18, p1. 1, fig. 12; Smith, 1957, p. 154, pl. 19, fig. 4a, b; Mallory, 1959 , p. 125, pl. 27, fig. 8a, b, pl. 33, fig. $2 a, b$, pl. 36, fig. 3a, b; Delise, 1967, p. 25, pl. 1, fig. 6a, b; Gaston, 1974, p. 50, [hypotype examined].

Hypotype no. 00221, loc. PSUA0106. 
Dorothia spp.

Hypotype no. 00222, 10c. PSUA0110.

Poor preservation prevented accurate identification of forms which resemble Dorothia principiensis Cushman and Bermudez. More than one species may be represented.

Genus Karreriella Cushman, 1933

Karrerielza chapapotensis (Cole) var. monumentensis Mallory Karreriella chapapotensis (Cole) var. monumentensis Mallory, 1959, p. 126, pl. 5, fig. 3a-c.

Hypotype no. 00223, loc. PSUA0111.

Poorly preserved specimens of this variety are easily confused with Chiloguembelina cubensis Palmer. Karreriella chapapotensis var. monumentensis however, can be distinguished by flattened chambers and a slit-like aperture surrounded by a distinct lip whereas $C$. cubensis possesses a low broadly arched aperture and more inflated chambers. Karreriezla elongata Mallory Karrerielza elongata Mallory, 1959, p. 127, pl. 5, fig. 4 a-c; Tipton, Kleinpell, \& Weaver, 1973, p. 43, pl. 1, fig. 15a, b. Hypotype no. 00224, 10c. PSUA0109.

Karrerielza mediaaguaensis Mallory

Karreriella media-aguaensis Mallory, 1959, p. 127, pl. 5, fig. $5 a, b, 6 a, b$; Sullivan, 1962 , p. 255 , pl. 4, fig. 5a, b; Fairchild, Wesendunk, \& Weaver, 1969, p. 34, p1. 4 , fig. 2a, b; Gaston, 1974 , p. 50, [hypotype examined]. Hypotype no. 00225, loc. PSUA0131.

$$
\text { Karrerielza [?] sp. }
$$

Hypotype no. 00226, loc. PSUA0108.

Poorly preserved specimens seem best placed in this genus. 
Genus Martinottielza Cushman, 1933

Martinottiella eoceniaa Cushman \& Bermudez

Martinottiella eocenica Cushman \& Bermudez, Mallory, 1959, p. 128 , pl. 5, fig. 8a, b; Sullivan, 1962 , p. 255 , pl. 4, fig. 6a, b; Tipton, Kleinpell, \& Weaver, 1973, p. 43, pl. 1, fig. 14a, b; Thoms, 1975, pl. 1, fig. 6a, b.

Hypotype no. 00227, 10c. PSUA0116.

$$
\text { Martinottielza [?] sp. }
$$

Hypotype no. 00228 , loc. PSUA0131.

$$
\text { Genus Tritaxilina Cushman, } 1911
$$

Tritaxilina colei Cushman \& Siegfus

Tritaxilina colei Cushman \& Siegfus, 1935, p. 92, pl. 14, fig. 5, 6; Kleinpell, 1938, p. 194, pl. 3, fig. 5; Mallory, p. 128, p1. 27, fig. $9 a, b$; Weaver \& Weaver, 1962, p. 21, pi. 2, fig. 10, Weaver, 1962, p. 372, pl. 1, fig. 2a, b; Kleinpell \& Weaver, 1963, p. 168, pl. 4, fig. 3a-c; Rau, 1964 , p. G15, pl. 5, fig. 2; Delise, 1967, p. 25, pl. 1, fig. 7; Fairchild, Wesendunk, \& Weaver, 1969, p. 34, pl. 4, fig. 8a, b; Smith, 1971, p. 35, p. 2, fig. 8; Gaston, 1974, p. 51, [hypotype examined].

Hypotype no. 00229, 10c. PSUA0137.

$$
\text { Tritaxilina [?] sp. }
$$

Hypotype no. 00230 , 10c. PSUA0100.

Specimens from the basal Neskowin creek Section are badly recrystallized and are questionably placed in this genus.

$$
\text { Family SILICINIDAE }
$$

Genus Silicosigmoilina Cushman \& Church, 1929

silicosigmoilina californica Cushman \& Church

Silicosigmoilina californica Cushman \& Church, Smith, 1957, p. 155, pl. 19, fig. 8a, b, 12a, b; Mallory, 1959, p. 129, pl. 5, fig. 10a, b; Thoms, 1975, pl. 1, fig. 7.

Hypotype no. 00231 , loc. PSUA0137. 
Family MILIOLIDAE

\author{
Genus Quinqueloculina d'Orbigny, 1826 \\ Quinqueloculina goodspeedi [?] Hanna \& Hanna
}

[?] Quinqueloculina goodspeedi Hanna \& Hanna, 1924, p. 58, pl. 13, fig. 3, 4; Beck, 1943, p. 592, pl. 99, fig. 1, 2; Asano, 1958, pl. 11, fig. 2a, b; Rau, 1964, p. Gl5, pl. 5, fig. $1 \mathrm{a}-\mathrm{c}$.

Hypotype no. 00232 , 10c. PSUA0119.

The apertural characteristics and general outline of a specimen from the Neskowin Creek Section closely approches Q. goodspeedi, but the surface of the test is badly worn making the specific identification uncertain.

Quinqueloculina imperialis Hanna \& Hanna

Quinqueloculina imperialis Hanna \& Hanna, 1924, p. 58, pl. 13, fig. 7, 8, 10; Beck, 1943, p. 592, pl. 98, fig. 9, 10; Cushman \& Frizzell, 1943, p. 82, pl. 14, fig. 1; Rau, 1948, p. 159, pl. 27, fig. 12-14; Cushman, Stewart, \& stewart, 1947d, p. 74, pl. 9, fig. 3a-c; Asano, 1958, pl. 11, fig. 7a, b; Delise, 1967, p. 26, pl. 1, fig. 8; Gaston, 1974, p. 51, [hypotype examined].

Hypotype no. 00233 , 10c. PSUA0119.

$$
\text { Quinqueloculina triangularis d'orbigny }
$$

Quinqueloculina triangularis d'orbigny, Cushman \& Hanna, 1927, p. 57, pl. 6, fig. 8, 9; Smith, 1957, p. 155, pl. 19, fig. 7a-c; Mallory, 1959, p. 130 , pl. 36 , fig. 5a-c;

Sullivan, p. 256 , pl. 4, fig. 12a-c.

Hypotype no. 00234 , 10c. PSUA0123.

Quinquezocuzina spp.

Numerous fragments, particularly common in the basal Neskowin Creek Section are best referred to this genus. 
Genus Massizina Schlumberger, 1893

Massizina decorata Cushman

Massizina decorata Cushman, 1922, p. 143, pl. 34, fig. 7; Cushman, 1935, p. 13, pl. 3, fig. 14-16; Cushman \& McMasters, 1936 , p. 510 , pl. 74, fig. 8; Bergquist, 1942 , p. 22, pl. 1, fig. 26; Franklin, 1944, p. 308, pl. 45, fig. 7; Mallory, 1959, p. 131, pl. 39, fig. 7a, b.

Hypotype no. 00235, 10c. PSUA0107.

Genus Spiroloculina d'orbigny, 1826

Spiroloculina (?) cf. S. Zamposa Hussey

Spiroloculina? cf. S. Zamposa Hussey, Smith, 1957, p. 155, pl. 19, fig. 10a, b, lla, b; Mallory, 1959, p. 131, pl. 5, fig. 13a, b, pl. 36, fig. 6a, b.

Spiroloculina Zamposa Hussey, Sullivan, 1962, p. 256, pl. 4, fig. $10 \mathrm{a}, \mathrm{b}$.

Spiroloculina cf. S. Zamposa Hussey, Gaston, 1974, p. 52, [hypotype examined].

Hypotype no. 00237, 10c. PSUA0137.

Spiroloculina wilcoxensis Cushman \& Garrett

Spiroloculina wilcoxensis Cushman \& Garrett, Cushman \&

Simonson, 1944, p. 194, pl. 30, fig. 4-6; Detling, 1946, p. 352, pl. 46, fig. 3; Hornaday, 1961, p. 181, pl. 2, fig. 3; Sullivan, 1962, p. 257, pl. 4, fig. 9; Weaver \& Molander, 1964, p. 180, pl. 3, fig. 3; Phillips, Tipton, \& Watkins, 1974, pl. l, fig. 1.

Spiroloculina cf. S. Zamposa Hussey, Weaver \& Weaver, 1962, p. 21, pl. 2, fig. 12 .

[?] Spiroloculina wiZcoxensis Cushman \& Garrett, Gaston, 1974, p. 52, [hypotype examined].

Hypotype no. 00236 , 10c. PSUA0127.

Genus Trizoculina d'Orbigny, 1826

Triloculina tricarinata d'orbigny

Trizoculina tricarinata d'Orbigny, Cushman \& Henbest, 1940, pl. 9, fig. 7; Todd, 1957a, pl. 86, fig. 15a, b; Todd, 1970 , p. A4, pl. 1, fig. 8 . 
Triloculina trigonula (Lamark), Howe, 1939, p. 39, pl. 3, fig. 1,2 .

Hypotype no. 00238 , loc. PSUAO100.

Trizoculina tricarinata is characterized by a sharply

angled, triangular shaped test with flat sided chambers. To the author's knowledge this species has not been recorded from Eocene deposits on the West Coast.

Triloculina [?] sp.

Hypotype no. 00239, loc. PSUA0100.

These incomplete specimens probably represent $T$. tricarinata.

Family OPHTHALMIDIIDAE

Genus Cornuspira Schultze, 1854

Cornuspira byramensis Cushman

Cornuspira lewisensis Beck, 1943, p. 594, pl. 101, fig. 4, 5. Cornuspira byramensis Cushman, Cushman \& Frizzell, 1943,

p. 83 , pl. 14, fig. 4; Rau, 1948, p. 160 , pl. 28, fig. 10, 11.

Hypotype no. 00240, loc. PSUA0117.

Two species of the genus Cornuspira were recorded from the Neskowin Creek Section. The nature of the periphery forms the basis for separating the two; $C$. byramensis has a rounded periphery and is circular in profile while $C$. olygogyra has flattened sides and is rectangular in section. Cornuspira byramensis is also more compressed than $C$. olygogyra. Cornuspira olygogyra Hantken

Cornuspira olygogyra Hantken, Cushman, 1935, p. 15, pl. 4, fig. 14; Coryell \& Embich, 1937, p. 293, pl. 41, fig. 15; Howe, 1939, p. 39, pl. 3, fig. 9, 10; Bergquist, 1942, p. 26 , pl. 2 , fig. 19 . 
Hypotype no. 00241, loc. PSUA0119.

See discussion under $C$. byramensis.

Family TROCHAMMINIDAE

Genus Trochammina Parker \& Jones, 1859

Trochammina albertensis Wickenden

Trochammina albertensis Wickenden, Cushman, 1946a, p. 50, pl. 15, fig. 7a-c; Sullivan, 1962, p. 256, pl. 4,

fig. 7a, b; Fairchild, Wesendunk, \& Weaver, 1969, p. 32, pl. 2, fig. 7a-c.

Hypotype no. 00242 , loc. PSUA011l.

Trochammina cf. T. globigeriniformis (Parker \& Jones)

Trochammina cf. T. globigeriniformis (Parker \& Jones), Smith,

1957, p. 156, pl. 19, fig. 16a-c; Mallory, 1959, p. 133, pl. 5, fig. 16 .

Trochammina globigeriniformis (Parker \& Jones), Gaston, 1974,

p. 52, [hypotype examined].

Hypotype no. 00243 , loc. PSUA0131.

Crushed individuals are conspecific with what has been referred to as T. cf. T. globigeriniformis in West coast literature.

Trochammina sp.

Hypotype no. 00244 , loc. PSUA0131.

Family LAGENIDAE

Genus Robulus Montfort, 1808

Robulus alatolimbatus (Gumbel)

Cristellaria alato-limbata (Gumbel), Cushman \& Applin, 1926,
p. 171, pl. 8, fig. 8. 
Robulus alato-limbatus (Gumbel), Cushman, 1935, p. 15, pl. 6, fig. 2a, b; Coryell \& Embich, 1937, p. 299, pl. 41, fig. 16; Howe, 1939, p. 40, pl. 4, fig. 18; Bergquist, 1942 , p. 26, pl. 3, fig. 7; Wilson, 1954, p. 133, pl. 14, fig. 3a, b; Todd, 1975a, pl. 64, fig. 24a, b; Mallory, 1959 , p. 133 , pl. 6, fig. 16a, b, pl. 27, fig. 1la, b; Hornaday, 1961, p. 182 , pl. 2, fig. 1la, b, 12a, b, 13a, b; Sullivan, 1962, p. 257, pl. 7, fig. 9a, b; Weaver \& Weaver, 1962 , p. 22, pl. 3, fig. 2a, b, 3; Weaver \& Molander, 1964 , p. 180, pl. 3, fig. 6a-c; Delise, 1967, p. 27, pl. 1, fig. 9a, b; Thoms, 1975, pl. l, fig. 23a, b.

Lenticulina alato-limbata (Gumbel), Fairchild, Wesendunk, \& Weaver, 1969, p. 40, pl. 5, fig. 15a, b; Tipton, Kleinpell, \& Weaver, 1973, p. 44, pl 2, fig. la, b.

Lenticulina alatolimbata (Gumbel), Smith, 1971, p. 40, pl. 3, fig. $3 a, b$.

[?] Robulus alato-limbata (Gumbel), Gaston, 1974, p. 53, [hypotype examined].

Hypotype no. 00245, loc. PSUA0108.

Robulus alotolimbatus is characterized by a small closecoiled test, approximately seven chambers in the last whorl, a large central umbo, and a narrow peripherial keel.

$$
\text { Robulus alatolimbatus [?] (Gumbel) }
$$

Hypotype no. 00246 , 10c. PSUA0137.

Several poorly preserved specimens with a large central umbo resemble $R$, alatolimbatus but are much larger and have a greater number of chambers in the last whorl.

Robulus arcuatostriatus (Hantken) var. carolinianus Cushman Robulus arcuato striatus (Hantken) var. carolinianus Cushman, Cushman, 1935, p. 17, pl. 6, fig. 6a, b; Coryell \& Embich, 1937 , p. 299, pl. 41, fig. 18; Bergquist, 1942, p. 27, pl. 2, fig. 23; Wilson, 1954, p. 133, pl. 13, fig. 4a, b, $5 a$, b; Mallory, 1959, p. 134, pl. 6, fig. 9a, b, pl. 27, fig. 12a, b; Sullivan, 1962, p. 257, pl. 7, fig. 8a, b; Weaver \& Weaver, 1962, p. 22, pl. 3, fig. 4a, b; Gaston, 1974 , p. 53, [hypotype examined]. 
Robulus cf. R. arcuato-striatus carolinianus Cushman, Cushman \& Simonson, 1944, p. 194, pl. 30, fig. 10.

Hypotype no. 00247 , 10c. PSUA0111.

This variety is distinguished by a large close-coiled test, strongly curved sutures, seven to nine chambers in the last whorl, and a radiate aperture. Robulus articulatus (Reuss) var. texanus (Cushman \& Applin) Cristelzaria articulata Reuss var. texana Cushman \& Applin, 1926 , p. 170, pl. 8, fig. 1, 2 .

Robulus articulatus (Reuss) var. texanus (Cushman \& Applin), Cushman \& Dusenbury, 1934, p. 53, pl. 7, fig. 2, 3; Cushman, 1935, p. 16, pl. 4, fig. 16, 17; Bergquist, 1942, p. 28, pl. 3, fig. 6; Cushman \& Frizzell, 1943, p. 83, pl. 14, fig. 10; Cushman \& Simonson, 1944, p. 194, pl. 30 , fig. 7; Mallory, 1959, p. 135, pl. 6, fig. 13a, b; Kleinpell \& Weaver, 1963, p. 169, pl. 4, fig. 5a, b; Weaver Molander, 1964, p. 180, pl. 3, fig. 7a, b; Gaston, 1974, p. 54, [hypotype examined]; Thoms, 1975, pl. 1, fig. 25 .

Robulus texanus (Cushman \& Applin), Beck, 1943, p. 595, pl. 103, fig. 1, 2, 4, 5; Rau, 1948, p. 163, pl. 29, fig. 16, 17; Todd, 1957a, pl. 64, fig. 20.

Lenticulina articulata (Reuss) var. texana (Cushman \& Applin), Fairchild, Wesendunk, \& Weaver, 1969, p. 41, pl. 5, fig. 19a, b.

Hypotype no. 00248 , 10c. PSUA0104.

As an adult, this variety is easily recognized by its chambers which uncoil reavealing the central portion of the test.

Robulus articulatus (Reuss) var. texanus [?] (Cushman \& Applin)

Hypotype no. 00249, 10c. PSUA0110.

A few fragmented specimens are questionalby referred to this variety. 
Robulus cf. R. chehalisensis Rau [of Smith]

Robulus cf. R. chehalisensis Rau, Smith, 1957, p. 157, pl. 20, fig. 3a, b; Weaver \& Weaver, 1962, p. $22, \mathrm{pl} .3$, fig. $5 a, b$.

Hypotype no. 00250, loc. PSUA0117.

Specimens from the Neskowin Creek Section appear conspecific with that figured by Smith.

\section{Robulus chiranus Cushman \& Stone}

Robulus chiranus Cushman \& Stone, Cushman, Stewart, \&

Stewart, 1947 e, p. 98 , pl. 12, fig. 5a, b; Cushman, Stewart, \& Stewart, 1949, p. 130, pl. 14, fig. 3a, b; Cushman \& Stainforth, 1951, p. 146, pl. 25, fig. 41; Hornaday, 1961, p. 182, pl. 3, fig. la, b, 2a, b, 4a, b; Sullivan, 1962, p. 257, pl. 7, fig. 1, 2a, b; Kleinpell \& Weaver, 1963, p. 169, pl. 4, fig. 6; Weaver \& Molander, 1964, p. 181, pl. 4, fig. la, b; Delise, 1967, p. 27, pl. 1, fig. 10a, b.

Robulus aff. R. warmani Barbat \& von Estorff, Wilson, 1954, p. 135, pl. 14, fig. 5a, b.

Lenticulina chiranus (Cushman \& Stone), Smith, 1971, p. 41, pl. 3, fig. 4a, b; Tipton, Kleinpell, \& Weaver, 1973, p. 44, pl. 2, fig. 2a, b.

Hypotype no. 00251, loc. PSUA0133.

\section{Robulus coaledensis Detling}

Robulus coaZendensis Detling, 1946, p. 353, pl. 48,

fig. la, b; Cushman \& Stainforth, 1951, p. 146, pl. 25,

fig. 40; Mallory, 1959, p. 136, pl. 7, fig. 1la, b, pl. 8,

fig. 8a, b; Weaver \& Molander, 1964, p. 181, pl. 4,

fig. $2 a, b, 3 a, b$.

[?] Robulus coaledensis Detling, Gaston, 1974, p. 54, [hypotype examined].

Hypotype no. 00252, loc. PSUA0123.

Robulus coaledensis is large, contains ten to eleven chambers in the final whorl and has markedly curved sutures. 
Robulus cf. R. coaledensis Detling

Hypotype no. 00253 , loc. PSUA0122.

Two specimens from the Neskowin Creek Section resemble R. coaledensis in that they have strongly curved sutures, but are smaller, thicker in peripheral view, and have only eight chambers in the last whorl.

\section{Robulus inornatus (d'orbigny)}

Robulus inornatus (d'Orbigny), Cushman \& Hanna, 1927, p. 51, pl. 4, fig. 4, 6; Cushman \& Barksdale, 1930, p. 62, pl. 11, fig. 2a, b, 3; Beck, 1943, p. 595, pl. 104, fig. 1-4, 10, 14; Cooper, 1944, p. 350, pl. 55, fig. 26, 27; Detling, 1946, p. 353 , pl. 47, fig. 4, 5; Cushman, Stewart, \& Stewart, 1947c, p. 60, pl. 7, fig. 3a, b; Cushman, Stewart, \& Stewart, $1947 \mathrm{~d}$, p. 74, pl. 11, fig. 6a, b; Cushman, Stewart \& Stewart, $1947 \mathrm{e}$, p. 97, pl. 13, fig. 2a, b; Wilson, 1954, p. 134, pl. 13, fig. 6a, b; Smith, 1956, p. 88, pl. 9, fig. 7a, b, 8a, b; Smith, 1957, p. 157, pl. 20, fig. $7 a, b, 8 a, b, 9 a, b, 10 a, b, 11 a, b ;$ Mallory, 1959 , p. 137, pl. 7, fig. 15a, b, pl. 40, fig. 5a, b; Hornaday, 1961, p. 182, pl. 2, fig. 5a, b; Sullivan, 1962, p. 258, pl. 5, fig. 5a, b; Weaver, 1962, p. 372, pl. 2, fig. 3a, b; Kleinpell \& Weaver, 1963, p. 169, pl. 4, fig. 7a, b, 8; Weaver \& Molander, 1964, p. 181, pl. 4, fig. $6 a, b, 7 a, b$.

Robulus inornata (d'Orbigny), Cushman \& Schenck, 1928, p. 307, pl. 42, fig. 3a, b.

Lenticulina inornata (d'Orbigny), Fairchild, Wesendunk, \& Weaver, 1969, p. 42, pl. 6, fig. 1; Tipton, Kleinpell, \& Weaver, 1973, p. 45, pl. 2, fig. 9a, b.

[?] Robulus inornatus (d'orbigny), Gaston, 1974, p. 55, [hypotype examined].

Hypotype no. 00254 , loc. PSUA0111.

This widely recorded species is characterized by five to seven chambers in the final whorl, sutures which are tangent to a large prominent umbo, and a slit-like aperture. 


\section{Robulus inornatus [?] (d'orbigny)}

Hypotype no. 00255, 10c. PSUA0105.

Numerous poorly preserved specimens, similar to $R$. inornatus, are included within this designation.

Robulus kincaidi Beck

Robulus kincaidi Beck, 1943, p. 595, pl. 102, fig. 1, 7;

Mallory, 1959, p. 137, pl. 6, fig. 17a, b; Sullivan, 1962, p. 259, pl. 7, fig. 4a, b; Gaston, 1974, p. 56, [hypotype examined].

Robulus aff. R. kincaidi Beck, Smith, 1957, p. 158, pl. 20, fig. $6 a, b$.

Hypotype no. 00256, loc. PSUA0125.

This species differs recognizably from other members of the genus in that the last chamber makes up nearly one-half of the test.

Robulus cf. R. kincaidi Beck

Hypotype no. 00257, 10c. PSUA0133.

Inflated specimens compare favorably with $R$. kincaidi but differ in that the final chamber does not make up one-half of the test.

\section{Robulus limbosus (Reuss) var. hockleyensis \\ (Cushman \& Applin)}

Cristellaria limbosa (Reuss) var. hockleyensis Cushman \& Applin, 1926, p. 171, pl. 8, fig. 3a, b, 4;

Robulus limbosus (Reuss) var. hockleyensis (Cushman A Applin), Cushman \& Dusenbury, 1934, p. 52, pl. 7, fig. 1; Cushman, 1935, p. 16 , pl. 4, fig. 15a, b, pl. 6, fig. 3a, b;

Bergquist, $1942, \mathrm{p} .31$, pl. 2, fig. 22; Cushman \& Simonson, 1944 , p. 194, pl. 30, fig. 9; Rau, 1948, p. 162, pl. 29, fig. 11, 12; Smith, 1956, p. 89, pl. 10, fig. 5a, b; Mallory, 1959, p. 139, pl. 6, fig. 15a, b; Weaver \& Weaver, 1962 , p. 23, pl. 4, fig. 5a, b; Gaston, 1974, p. 56, [hypotype examined]. 
Lenticulina limbosa (Reuss) var. hockleyensis (Cushman \& Applin), Fairchild, Wesendunk, \& Weaver, 1969, p. 42, pl. 6, fig. 2 .

Hypotype no. 00258, 10c. PSUA0110.

Robulus limbosus (Reuss) var. hockleyensis [?] (Cushman \& Applin)

Hypotype no. 00259, 10c. PSUA0125.

Incomplete individuals that resemble this species are included here.

Robulus mayi Cushman \& Parker

Robulus mayi Cushman \& Parker, Bergquist, 1942, p. 31, pl. 3, fig. 2; Mallory, 1959, p. 139, pl. 6, fig. lia, b; Sullivan, 1962, p. 259, pl. 7, fig. 5a, b; Weaver \& Weaver, 1962, p. 23, pl. 4, fig. 6a, b; Gaston, 1974, p. 56, [hypotype examined].

Lenticulina mayi (Cushman \& Parker), Tipton, Kleinpel1, \& Weaver, 1973, p. 45, pl. 2, fig. 10a, b.

Hypotype no. 00260, loc. PSUA0137.

Robulus mayi [?] Cushman \& Parker

Hypotype no. 00261, loc. PSUA0110.

These specimens are similar to $R$. mayi in that the final chambers become elongate but poor preservation prevents positive identification.

Robuzus cf. R. midwayensis (Plummer)

Hypotype no. 00262 , loc. PSUA0137.

A few specimens with raised sutures resemble $R$. midwayensis but lack the central boss and have only six chanbers in the final whorl. 
Robulus propinquus cowlitzensis Beck

Robulus propinquus cowlitzensis Beck, 1943, p. 595, pl. 104, fig. 6, 12; Sullivan, 1962, p. 259, pl. 6, fig. 6, 7a, b, $8 \mathrm{a}, \mathrm{b}$; Weaver \& Weaver, 1962, p. 23, pl. 4, fig. 7; Weaver \& Molander, 1964, p. 181, pl. 4, fig. 8a, b; Gaston, 1974 , p. 57, [hypotype examined].

Lenticulina propinqua cowlitzensis Beck, Fairchild, Wesendunk, \& Weaver, 1969, p. 42, pl. 6, fig. 9a, b; Tipton, Kleinpeli, \& Weaver, 1973, p. 46, pl.2, fig. lla, b.

Hypotype no. 00263, 10c. PSUA0123.

Robulus pseudovortex cole

Robulus pseudovortex Cole, Cushman \& McMasters, 1936, p. 510, pl. 74, fig. 12a, b; Franklin, 1944, p. 310, pl. 45, fig. 12; Smith, 1957, p. 158, pl. 20, fig. 12a, b, 13a, b; Mallory, 1959, p. 141, pl. 7, fig. 2, 3a, b, pl. 27, fig. 13a, b; Weaver \& Weaver, 1962, p. 24, pl. 4, fig. 8a, b; Gaston, 1974, p. 57, [hypotype examined] ; Thoms, 1975, pl. 1, fig. $27 a, b$.

Lenticulina pseudovortex (Cole), Fairchild, Wesendunk, \&

Weaver, 1969, p. 42, pl. 7, fig. 6a, b, 1la, b; Smith, 1971, p. 43, pl. 3, fig. 7a, b.

Hypotype no. 00264 , loc. PSUA0112.

$$
\text { Robulus cf. R. pseudovortex Cole }
$$

Hypotype no. 00265, loc. PSUA0133.

Numerous specimens with recurved sutures compare favorably with this species but are larger, not as close-coiled, and average five or more chambers in the last whorl.

Robulus cf. R. terryi Coryell \& Embich

Robulus cf. R. terryi Coryell \& Embich, Smith, 1957, p. 159, pl. 21, fig. la, b; Mallory, 1959, p. 141, pl. 6, fig. la, b; Sullivan, 1962, p. 260, pl. 7, fig. 6a, b; Weaver \& Weaver, 1962, p. 24, pl. 5, fig. 2a, b; Weaver \& Molander, 1964, p. 182, pl. 5, fig. 2a, b.

Lenticulina cf. L. terryi (Coryell \& Embich), Fairchild, Wesendunk, \& Weaver, 1969, p. 43, pl. 7, fig. 8a, b; Tipton, Kleinpell, \& Weaver, 1973, p. 46, pl. 2, fig. 8a, b. 
Lenticulina terryi (Coryell \& Embich), Smith, 1971, p. 43, pl. 3, fig. 8a, b.

Robulus terryi Coryell \& Embich, Gaston, 1974, p. 57,

[hypotype examined].

Hypotype no. 00266, loc. PSUA0104.

Coryell and Embich (1937, p. 299) originally described this species as:

Test free, close-coiled, with a wide carina; chambers 12 or 14 in number becoming narrower toward the umbilical area of the test; due to shortening, the chambers fail to cover preceding whorl entirely; sutures limbate, but not raised; slightly curved; orifice robuline on the last septal face.

Subsequently, authors have referred to a similar form occurring in West Coast deposits as $R$. cf. $R$ terryi. The most characteristic feature that links the two forms is the shortening of the later chambers, allowing the earlier chambers to be viewed through a transparent umbo. In all other features the two appear conspecific except for the number of chambers in the last coil (Tipton, Kleinpell, and Weaver, 1973, p. 46). Mallory reports this species, $R$. cf. $R$. terryi, as having five to 13 final chambers while most other authors figure a form with between six and ten chambers (Smith, 1957, pl. 21, fig. la, b; Sullivan, 1962, pl. 7, fig. 6a, b; Weaver and Weaver, 1962, pl. 5, fig. 2a, b; Weaver and Molander, 1964, pl. 5, fig. 2a, b; Fairchild, Wesendunk, and Weaver, 1969, pl. 7, fig. 8a, b; Smith, 1971, pl. 3, fig. 8a, b). Some authors have synonymized the two (Smith, 1971, p. 43; Gaston, 1974, p. 57) but further study of the primary types is suggested for conformation of their equivalence. 
Robulus weaveri Beck

Robulus weaveri Beck, 1943, p. 595, pl. 103, fig. 3, 8; Rau, 1948, p. 162, pl. 28, fig. 18, 19; Mallory, 1959, p. 143, pl. 7, fig. 14a, b; Weaver \& Weaver, 1962, p. 24, pl. 5, fig. 4a, b: Gaston, 1974, p. 58, [hypotype examined].

Hypotype no. 00267, 10c. PSUA0108.

Robulus weaveri [?] Beck

Hypotype no. 00268, loc. PSUA0110.

Specimens with tests higher than broad resemble $R$. weaveri but are poorly preserved and questionably assigned to this species.

\section{Robulus welchi Church}

Robulus welchi Church, 1931, pl. C, fig. 13, 14; Beck, 1943, p. 596, pl. 102, fig. 4, 8; Cushman \& Simonson, 1944 , p. 195, pl. 30, fig. 11; Detling, 1946, p. 353, pl. 48, fig. 2a, b; Cushman, Stewart, \& Stewart, 1947e, p. 97, pl. 12, fig. 4a, b; Cushman, stewart, \& Stewart, 1949, p. 130, pl. 14, fig. 8a, b; Smith, 1957, p. 159, pl. 21, fig. $4 a, b, 5 a, b, 6 a, b ;$ Mallory, 1959, p. 143, pl. 7, fig. 8a, b; Hornaday, 1961, p. 183, pl. 3, fig. 3a, b; Weaver \& Weaver, 1962 , p. 24 , pl. 5, fig. 5a, b; Rau, 1964, pl. 5, fig. 6a, b; Weaver \& Molander, 1964, p. 182, pl. 5, fig. 3a, b, 4a, b; Gaston, 1974, p. 58, [hypotype examined]; Thoms, 1975, pl. 2, fig. la, b.

Hypotype no. 00269 , 10c. PSUA0123.

$$
\text { Robuzus spp. }
$$

This designation is used to include numerous poorly preserved specimens that are not identifiable at a specific level. More than one species is represented.

$$
\text { Genus Lenticulina Lamarck, } 1804
$$

Lenticulina cf. L. convergens (Bornemann) [of Mallory] Lenticulina convergens (Bornemann) (?), Cushman \& Barksdale, 1930, p. 64, pl. 11, fig. 8a, b. 
Lenticulina (?) convergens (Bornemann), Cushman \& Dusenbury, 1934, p. 54, pl. 7, fig. 7a, b.

Lenticulina convergens (Bornemann), Beck, 1943, p. 596, pl. 104, fig. 5, 9; Fairchild, Wesendunk, \& Weaver, 1969, p. 41, pl. 5, fig. 1la, b.

[Non] Lenticulina cf. L. convergens (Bornemann), Cushman \& Simonson, 1944, p. 195, pl. 30, fig. 12; Sullivan, 1969, p. 261, pl. 8, fig. 2a, b.

Robulus convergens (Bornemann), Smith, 1957, p. 157, pl. 20, fig. $5 a, b$.

Lenticulina cf. L. convergens (Bornemann), Mallory, 1959, p. 145, pl. 8, fig. 13a, b, 14a, b, pl. 27, fig. 15a, b. Hypotype no. $00270,10 c$. PSUA0106.

Two individuals from the Neskowin Creek Section are conspecific with those figured by Mallory.

$$
\begin{aligned}
& \text { Genus PZanularia Defrance, } 1824 \\
& \text { PZanuZaria markZeyana Church }
\end{aligned}
$$

Planularia markleyana Church, 1931, pl. A, fig. 6, pl. B, fig. 1, 10; Rau, 1948, p. 164, p1. 29, fig. 19; Mallory, 1959 , p. 147, pl. 9, fig. 5a, b; Weaver \& Weaver, 1962, p. 25 , pl. 6 , fig. $4 a, b$.

Hypotype no. 00271 , 10c. PSUA0127.

Planularia cf. $P$. ouachitaensis Howe \& Wallace [of Mallory]

Planularia cf. P. ouachitaensis Howe \& Wallace, Mallory, 1959, p. 147, pl. 9, fig. $6 a, b$.

Hypotype no. 00272 , loc. PSUA0123.

Specimens from the Neskowin Creek Section have a slight keel but are otherwise identical to Mallory's figure. 
PZanuZaria cf. P. truncana (Gumbel)

Hypotype no. 00273, loc. PSUA0110.

A single specimen from the Neskowin Creek Section differs from $P$. truncana in having fewer chambers and a flattened apertural face.

$$
\text { PZanuZaria [?] sp. }
$$

Hypotype no. 00274 , 10c. PSUA0106.

Poorly preserved, compressed individuals that possess keeled margins seem best placed in this genus. More than one species may be represented.

Genus Marginulina d'Orbigny, 1826

Marginulina adunca (Costa)

Psecadium aduncum (Costa), Galloway \& Morrey, 1931, p. 336, pl. 37, fig. 13.

Marginulina adunca (Costa), Mallory, 1959, p. 148, pl. 9, fig. Ila, b, 12a, b; Weaver \& Molander, 1964, p. 183, pl. 6, fig. 4; Fairchild, Wesendunk, \& Weaver, 1969, p. 44, pl. 8, fig. 1; Smith, 1971, p. 44, pl. 4, fig. 6; Gaston, 1974, p. 59, [hypotype examined].

Hypotype no. 00275, loc. PSUA0106.

$$
\text { Marginulina adunca [?] (Costa) }
$$

Hypotype no. 00276, loc. PSUA0113.

Three internal molds are referable to this species. Marginulina cf. M. alazanensis Nuttall

Hypotype no. 00277, loc. PSUA0110.

These specimens differ from $M$. alazanensis (Sullivan, 1962, p. $261, \mathrm{pl} .8$, fig. $8 \mathrm{a}, \mathrm{b})$ in possessing oblique sutures and more inflated chambers. 
Marginulina eximia Neugeboren

Marginulina eximia Neugeboren, Bergquist, 1942, p. 36, pl. 4, fig. 8; Beck, 1943, p. 597, pl. 104, fig. 15, 16; Sullivan, 1962 , p. 262, pl. 8, fig. 9a, b; Weaver \& Molander, 1964, p. 183, pl. 6, fig. 5; Fairchild, Wesendunk, \& Weaver, 1969 , p. 44, pl. 8, fig. 4; Smith, 1971, p. 45, pl. 4, fig. 8; Tipton, Kleinpell, Weaver, 1973, p. 46, pl. 3 , fig. la, b; Gaston, 1974, p. 59, [hypotype examined]. Marginulina sp. Cushman \& Simonson, 1944, p. 196, pl. 30, fig. 15, pl. 31, fig. 1-4.

Marginulina eximia Neugeboren (?), Mallory, 1959, p. 149, pl. 10, fig. la-c.

Hypotype no. 00278, loc. PSUA0104. Marginulina cf. M. eximia Neugeboren Hypotype no. 00279 , 10c. PSUA0108.

A single specimen from the Neskowin Creek Section resembles M. eximia but possesses a much narrower test. Marginulina holmesi Gaston [nomen nudum] Marginulina holmesi Gaston, 1974, p. 60, fig. on p. 60. Hypotype no. 00280 , 10c. PSUA0107.

Marginulina holmesi was described from the Yamhill Formation in an unpublished master's thesis. An early compressed coiled portion followed by an elongate rectilinear stage distingishes this species from other members of the genus. It resembles $M$. jacksonensis (Cushman and Applin) and $M$. sublituus (Nuttall) (Bergquist, 1942, p. 37-38, pl. 126, fig. 2, 3) except that the coiled portion of the test is at right angles to the rectilinear stage. The holotype was examined and Neskowin Creek specimens are conspecific. 
Marginulina huneri [?] Howe

[?] Marginulina huneri Howe, 1939 , p. 43, pl. 6, fig. 7; Mallory, 1959, p. 150, pl. 27, fig. 18a, b.

Hypotype no. 00281, 10c. PSUA0132.

One broken, pyritized specimen from the Salmon River Section is questionably referred to this species.

Marginulina cf. M. inconspicua Hussey [of Sullivan]

Marginulina cf. M. inconspicua Hussey, Sullivan, 1962, p. 262,

pl. 8, fig. 6a, b; Weaver \& Molander, 1964, p. 183, pl. 6,

fig. 6 .

Marginulina inconspicua Hussey, Fairchild, Wesendunk, Weaver, 1969, p. 45, pl. 8, fig. 3a, b; Gaston, 1974, p. 61,

[hypotype examined].

Hypotype no. 00282, 10c. PSUA0133.

A single specimen from the Salmon River Section resembles this form.

\section{Marginuzina munda [?] Cushman}

[?] Marginulina munda Cushman, Cushman \& Deadrick, 1944, p. 331, pl. 51, fig. 4; Cushman, 1946a, p. 60, pl. 21, fig. 2, 3; Frizzell, 1954, p. 85, pl. 9, fig. 5; Sullivan, 1962 , p. 262, pl. 8, fig. 5a, b; Fairchild, Wesendunk, \& Weaver, 1969 , p. 45 , pl. 8, fig. 18.

Hypotype no. 00283, loc. PSUA0123.

Two specimens from the Neskowin Creek Section, although very similar to this species, are pyritized and too poorly preserved to permit positive identification.

$$
\text { Marginulina regularis d'orbigny }
$$

Marginulina regularis d'orbigny, Mallory, 1959, p. 150, pl. 10, fig. 6a, b.

Hypotype no. 00284 , loc. PSUA0137. 
Marginulina subbulzata Hantken

Marginulina subbullata Hantken, Coryell Embich, 1937,

p. 297, pl. 42, fig. 2; Cushman, Stewart, \& Stewart, 1949,

p. 131, pl. 14, fig. 10a, b; Mallory, 1959, p. 151, p1. 9,

fig. 13a, b, 14a, b, 15a, b; Weaver \& Weaver, 1962, p. 26, pl. 6, fig. 11; Weaver \& Molander, 1964, p. 183, pl. 6, fig. 7; Gaston, 1974, p. 61, [hypotype examined] ; Thoms, 1975, pl. 1, fig. 19 .

Hypotype no. 00285 , 10c. PSUA0106.

$$
\text { Marginulina sp. }
$$

Hypotype no. $00286,10 c$. PSUA0112.

Three specimens, similar to M. Laeviuscula Cushman and Bermudez (?) of Mallory (1959, p. 150, p1. 10, fig. 5a, b), are insufficiently preserved for specific identification.

$$
\text { Marginulina [?] sp. }
$$

Hypotype no. 00287, loc. PSUA0137.

A single specimen resembling $M$. sp. of Fairchild, Wesendunk, and Weaver $(1969$, p. 45, pl. 8, fig. 7) differs in possessing a keel on the outer margin of the test. This specimen may also represent the genus Saracenaria.

$$
\text { Genus Vaginulinopsis Sylvestri, } 1904
$$

Vaginulinopsis cf. V. asperuliformis (Nuttall) Mallory Vaginulinopsis cf. V. asperuliformis (Nuttall), Mallory, Weaver, 1962, p. 375 , pl. 2, fig. 7 .

Hypotype no. 00288, loc. PSUA0132.

Pyritized specimens from the Salmon River Section possess the same longitudinal costae which parallel the periphery of the test that Weaver figured (1962, p1. 2, fig. 7) and Mallory described (1959, p. 155). Further study of the variation within $V$. asperuliformis may show the two to be synonymous. 
Vaginulinopsis mexicana (Cushman) var. nudicostata (Cushman \& G. D. Hanna)

Robulus mexicanus (Cushman) var. nudicostatus (Cushman \& G. D. Hanna), Cushman \& M. A. Hanna, 1927, p. 50, pl. 4, fig. 2; Cushman \& McMasters, 1936, p. 511, pl. 74, fig. 15, 16 .

Vaginulinopsis mexicana (Cushman) var. nudicostata (Cushman \& G. D. Hanna), Smith, 1957, p. 162, pl. 22, fig. 3a, b; Mallory, 1959, p. 157, pl. 28, fig. la, b, pl. 40, fig. 2a, b, 3; Thoms, 1975, pl. 2, fig. 4a, b.

[?] Vaginulinopsis mexicana (Cushman) var. nudicostata (Cushman \& G. D. Hanna), Gaston, 1974, p. 62, [hypotype examined].

Hypotype no. $00289,10 c$. PSUA0100.

Vaginulinopsis saundersi (Hanna \& Hanna)

Cristellaria saundersi Hanna \& Hanna, 1924, p. 61, pl. 13, fig. $5,6,15$.

Vaginulinopsis saundersi (Hanna \& Hanna), Beck, 1943, p. 598, pl. 105, fig. 1, 2, 4, 5, 10; Cushman \& Stainforth, 1951, p. 147, pl. 26, fig. 8; Smith, 1957, p. 162, pl. 22, fig. la, b, 2a, b; Mallory, 1959, p. 157, pl. 11, fig. $10 a, b$; Kleinpell \& Weaver, 1963, p. 170, pi. 6 , fig. 6; Weaver \& Molander, 1964, p. 184, pl. 6, fig. 9, Gaston, 1974, p. 63, [hypotype examined].

Hemicristellaria saundersi (Hanna \& Hanna), Asano, 1958, pl. 10, fig. 3, 4 .

Hypotype no. 00290, loc. PSUA0103.

Vaginulinopsis sp.

Hypotype no. 00291 , loc. PSUA0102.

One specimen from the lower Neskowin Creek Section is badly recrystallized and can only be identified on a generic level.

Genus Astacolus Montfort, 1808

$$
\text { Astacolus [?] sp. }
$$

Hypotype no. 00292 , loc. PSUA0123.

Compressed individuals seem referable to this genus. 


\section{Genus Dentalina d'Orbigny, 1826 \\ Dentalina angusticostata Cushman}

Dentalina angusticostata Cushman, Cushman, 1946a, p. 70 , pl. 24, fig. 7, 8; Frizzell, 1954, p. 86, pl. 9, fig. 30,31 .

? Dentalina angusticostata Cushman, Mallory, 1959, p. 161, pl. 12, fig. $3 a, b$.

Hypotype no. $00293,10 c$. PSUA0116.

Figures of $D$. angusticostata and $D$. delicatula Cushman (Cushman, 1946a, p. 70, pl. 25, fig. 1-6) are almost impossible to separate. Both have numerous longitudinal costae independent of the sutures, slender elongate tests, limbate sutures in the early portions of the test and depressed sutures between later chambers. The only differences appear to be in the length and inflation of the individual chambers; D. angusticostata has somewhat elongate inflated chambers while $D$. delicatula has smaller less inflated chambers. Numerous specimens from the Neskowin Creek Section have been referred to $D$. angusticostata on this basis. Further study of the variation between these two species however, may show the two to be synonymous.

\section{Dentalina angusticostata [?] Cushman}

Hypotype no. 00294 , loc. PSUA0129.

Poorly preserved specimens are questionably placed in this designation. 


\section{Dentalina colei Cushman \& Dusenbury}

Dentalina colei Cushman \& Dusenbury, 1934, p. 54, pl. 7, fig. 10-12; Beck, 1943, p. 598, pl. 105, fig. 18; Mallory, 1959 , p. 162, pl. 12, fig. 9, pl. 41, fig. 3; Sullivan, 1962 , p. 263, pl. 9, fig. $6 a, b$; Weaver \& Weaver, 1962, p. 26, pl. 7, fig. 5; Weaver \& Molander, 1964, p. 184, pl. 6, fig. 11; Fairchild, Wesendunk, \&eaver, 1969, p. 37, pl. 5, fig. 17; Gaston, 1974, p. 63, [hypotype examined].

Hypotype no. 00295, 10c. PSUA0105.

$$
\text { Dentalina colei [?] Cushman \& Dusenbury }
$$

Hypotype no. $00296,10 c$. PSUA0129.

\section{Dentalina communis d'orbigny}

[Non] Nodosaria communis (d'orbigny), Bagg, 1905, p. 29 , pl. 5, fig. 2; Cushman, 1913, p. 54, pl. 28, fig. 1, 2; Cushman, 1918, p. 50, pl. 10, fig. 8.

Nodosaria communis (d'orbigny), Bagg, 1912, p. 54, pl. 16, fig. 8, 9, [not fig. 5]; Cushman, 1922, p. 129, pl. 30, fig. 4 .

Nodosaria (Dentalina) communis (d'Orbigny), Cushman \& M. A. Hanna, 1927, p. 4, p1. 4, fig. 11, 12 .

Dentalina communis d'Orbigny, Beck, 1943, p. 598, pl. 105, fig. 22; Detling, 1946, p. 353, pl. 48, fig. 4; Cushman, Stewart, \& Stewart, 1947 d, p. 76, pl. 9, fig. 9; Rau, 1948 , p. 166, pl. 29, fig. 2; Puri, 1953, p. 99, pl. 23, fig. 14; Frizzell, 1954, p. 87, pl. 9, fig. 39, 40; Smith, 1957, p. 165, pl. 22, fig. 9; Todd, 1957 a, pl. 65, fig. 4; Mallory, 1959, p. 162, pl. 12, fig. 11, [not pl. 41, fig. 6]; Weaver \& Weaver, 1962, p. 26, pl. 7, fig. 6; Todd, 1966, pl. 12, fig. 1; Gaston, 1974, p. 64, [hypotype examined].

Dentalina cf. D. communis d'orbigny, Cushman, Stewart, \& Stewart, 1949, p. 131, pl. 15, fig. 2 .

Hypotype no. 00297, 10c. PSUA0132.

Dentalina cf. D. communis d'orbigny

Nodosaria communis (d'orbigny), Bagg, 1912, p. 54, pl. 16, fig. 5, [not fig. 8, 9]; Cushman, 1918, p. 50, pl. 10, fig. 8 . 
Dentalina cf. D. communis d'orbigny, Weaver \& Molander, 1964, p. 184, pl. 6, fig. 12; Fairchild, Wesendunk, \& Weaver, 1969 , p. 38, pl. 5, fig. 3.

Hypotype no. 00298, 10c. PSUA0108.

This distinctive species is closely related to $D$. communis but differs in possessing more inflated chambers and oblique sutures.

\section{Dentalina consobrina d'orbigny}

[Non] Nodosaria consobrina (d'Orbigny), Bagg, 1905, p. 29, pl. 5, fig. 3; Bagg, 1912, p. 54, pl. 15, fig. 5a-d.

Nodosaria (Dentalina) consobrina (d'orbigny), Cushman \& M. A. Hanna, 1927, p. 52, pl. 4, fig. 7, 8.

Dentalina consobrina d'orbigny?, Cushman \& Schenck, 1928, p. 308, p1. 42, fig. 9-11.

Dentalina consobrina d'orbigny, Cushman \& Barksdale, 1930, p. 64 , pl. 11, fig. 9, 10; Kleinpell, 1938, p. 210, pl. 3, fig. 12, 14, 15, pl. 17, fig. 4; Wilson, 1954, p. 135, p1. 14, fig. 7; Todd, 1957a, pl. 65, fig. 9-11; Mallory, 1959 , p. 163, pl. 12, fig. 12, pl. 41, fig. 5; Hornaday, 1961 , p. 184, pl. 4, fig. 9; Sullivan, 1962, p. 263, p1.9, fig. 2, 3a, b; Kleinpell \& Weaver, 1963, p. 170, pl. 6, fig. 3, 4, pl. 7, fig. 3; Todd, 1970, p. A7, pl. 2, fig. 11; Gaston, 1974 , p. 65, [hypotype examined]; Thoms, 1975, pl. 1, fig. 13 .

Nodosaria consobrina (d'Orbigny), Galloway \& Morrey, 1931, p. 337, pl. 37, fig. 17; Bandy, 1944, p. 369, pl. 60,

fig. 12 ; Detling, 1946 , p. 353 , pl. 48 , fig. 5,6 .

Dentalina cf. D. consobrina d'Orbigny, Cushman \& Masters, 1936, p. 511, pl. 74, fig. 18-20.

Hypotype no. 00299 , loc. PSUA0116.

$$
\text { Dentalina consobrina [2] d'orbigny }
$$

Hypotype no. $00300,10 c$. PSUA0115.

Specimens that resemble this species but lack the initial bulbous chamber or basal spine are included here. 
Dentalina cooperensis Cushman

Dentalina cooperensis Cushman, Cushman, 1935, p. 20, pl. 8, fig. 3, 4; Coryell \& Embich, 1937, p. 298, pl. 42, fig. 8; Bergquist, 1942 , p. 42, pl. 5, fig. 3; Cushman \& Simonson, 1944 , p. 196, pl. 31, fig. 5-7; Wilson, 1954, p. 135 , pl. 14, fig. 8-10; Puri, 1957, p. 112, pl. 4, fig. 4; Todd, $1957 a$, pl. 65, fig. 3; Hornaday, 1961, p. 185, pl. 4, fig. 8a, b; Sullivan, 1962, p. 263, pl. 9, fig. 4a, b, $5 a, b$; Weaver \& Molander, 1964, p. 184, pl. 6, fig. 13; Fairchild, Wesendunk, Weaver, 1969, p. 38, pl. 5, fig. 4; Gaston, 1974, p. 65, [hypotype examined].

Hypotype no. $00301,10 c$. PSUA0112.

Although this species is similar in general outline to $D$. communis, it possesses less oblique sutures.

Dentalina dusenburyi Beck

Dentalina capitata (Boll) (?), Cushman \& Dusenbury, 1934, p. 56, pl. 7, fig. 20 .

Dentalina dusenburyi Beck, 1943, p. 599, pl. 105, fig. 20, 23; Cushman, Stewart, \& Stewart, 1947d, p. 76, pl. 10, fig. 1-3; Rau, 1948, p. 167, pl. 30, fig. 24; Smith, 1956, p. 90 , pl. 11, fig. 6; Asano, 1958, pl. 10, fig. 5, 6; Mallory, 1959, p. 163, pl. 12, fig. 14; Weaver \& Molander, 1964 , p. 184 , pl. 6, fig. 14; Delise, 1967, p. 31, pl. 2, fig. 11; Gaston, 1974, p. 66, [hypotype examined].

Hypotype no. $00302,10 c$. PSUA0130.

Dentalina eocenica Cushman (?) [of Mallory]

Dentalina eocenica Cushman ?, Mallory, 1959, p. 164, pl. 13, fig. 6 .

Hypotype no. 00303 , 10c. PSUA0119.

A few pyritized individuals resemble Mallory's figure.

Dentalina hexacostata Howe

Dentalina hexacostata Howe, 1939, p. 44, pl. 5, fig. 13.

Dentalina hexacostata Howe ?, Mallory, 1959, p. 164, pl. 12, fig. 15, pl. 28, fig. 2 .

Hypotype no. 00304 , 10c. PSUA0122. 
Dentalina cf. D. hexacostata Howe

Hypotype no. 00305, loc. PSUA0116.

Howe (1939, p. 44) originally described $D$. hexacostata as "uniform in diameter throughout its length" and "decorated with six longitudinal widely spaced costae". Several specimens from the Neskowin Creek and Salmon River Sections possess six to eight widely spaced costae and slightly inflated terminal chambers. In all other respects however, the specimens compare favorably with $D$. hexacostata.

\section{Dentalina jacksonensis (Cushman \& Applin)}

Nodosaria jacksonensis Cushman \& Applin, 1926, p. 170, pl. 7, fig. 14-16.

Dentalina jacksonensis (Cushman \& Applin), Cushman, 1935, p. 20, pl. 8, fig. 7-9; Coryell \& Embich, 1937, p. 298, p1. 42, fig. 8; Bergquist, 1942 , p. 43 , pl. 5, fig. 13; Cushman \& Simonson, $1944, \mathrm{p} .196, \mathrm{pl} .32$, fig. 8; Franklin, 1944 , p. 310, pl. 46, fig. 10; Todd, 1957a, pl. 65,

fig. 5-8; Mallory, 1959, p. 165, pl. 12, fig. 18; Gaston,

1974, p. 66 , [hypotype examined].

Hypotype no. 00306, loc. PSUA0116.

$$
\text { Dentalina kreyenhagenensis [?] Mallory }
$$

[?] Dentalina kreyenhagenensis Mallory, 1959, p. 165, pl. 12, fig. 23, 24, pl. 29, fig. 13, 14; Weaver \& Weaver, 1962, p. 27, pl. 7, fig. 8 .

Hypotype no. 00307, loc. PSUA0117.

Two fragmented specimens from the Neskowin Creek Section resemble Mallory's figure.

\section{Dentalina pauperata d'orbigny}

[Non] Nodosaria pauperata (d'Orbigny), Bagg, 1905, p. 32, pl. 5, fig. 8; Bagg, 1912, p. 57, pl. 16, fig. $2 \mathrm{a}-\mathrm{f}$; Cushman, 1913, p. 51, pl. 25, fig. 7 . 
Nodosaria pauperata (d'orbigny), Hanna, 1923, p. 322, pl. 58, fig. 3, 5.

Dentalina pauperata d'orbigny, Kleinpel1, 1938, p. 213,

pl. 11, fig. 6; Cushman, stewart, \& Stewart, 1947a, p. 14, p1. 1, fig. 10; Smith, 1956, p. 91, pl. 11, fig. 7,

Sullivan, 1962, p. 264, pl. 9, fig. 7; Kleinpell \& Weaver, 1963, p. 170, pl. 6, fig. 5; Fairchild, Wesendunk, \& Weaver, 1969, p. 38, pl. 5, fig. 5 .

Hypotype no. 00308, loc. PSUA0128.

Dentalina pauperata [?] d'orbigny

Hypotype no. 00309 , 10c. PSUA0132.

Incomplete specimens resembling $D$. pauperata are included here.

Dentalina cf. D. sherborni Bowen [of Mallory]

Dentalina cf. D. sherborni Bowen, Mallory, 1959, p. 166,

pl. 13, fig. 2; Weaver \& Weaver, 1962, p. 27, pl. 7,

fig. 9,10 .

Hypotype no. 00310, 10c. PSUA0111.

Individuals from the Neskowin Creek Section are conspecific with the specimen figured by Mallory.

Dentalina soluta Reuss

Nodosaria soluta (Reuss), Bagg, 1905, p. 34, pl. 5, fig. 11;

Bagg, 1912, p. 59, pl. 15, fig. 2a, b, pl. 16, fig. 7;

Cushman, 1913, p. 53, p1. 26, fig. 9-11; Cushman, 1926b,

p. 597, pl. 17, fig. 19, [not pl. 18, fig. 9].

[Non] Nodosaria soluta (Reuss), Hanna, 1923, p. 322, pl. 58, fig. 6.

Dentalina soluta Reuss, Todd, 1957a, pl. 65, fig. 14; Todd, 1957b, pl. 28, fig. 8 .

Dentalina soluta Reuss (?), Mallory, 1959, p. 167, pl. 13,

fig. 1, pl. 28, fig. 4.

Hypotype no. 00311, 10c. PSUA0129. 
Dentalina spinosa d'orbigny

Nodosaria spinosa (d'Orbigny), Galloway \& Morrey, 1939,

p. 339, pl. 38, fig. 4, [not fig. 5].

Dentalina spinosa d'Orbigny, Kleinpell, 1938, p. 215, pl. 4, fig. 3; Smith, 1956, p. 91, pl. 10, fig. 10; Smith, 1957, p. 165, pl. 22, fig. 10; Kleinpell \& Weaver, 1963, p. 170, p1. 6, fig. 8, 10, 11 .

[Non] Dentalina spinosa d'Orbigny, Mallory, 1959, p. 167, pl. 12, fig. 26; Sullivan, 1962, p. 264, pl. 9, fig. 17a, b, 18; Gaston, 1974, p. 67, [hypotype examined]. Hypotype no. 00312, 10c. PSUA0131.

Dentalina spinosa, as figured by d'orbigny (in Ellis and Messina) has short inflated chambers with prominent costae which are not continuous beyond the sutures. Individuals similar to $D$. spinosa but with elongate chambers and coarse spines are referred to in this report as Nodosaria cf. $N$. deliciae Martin and appear conspecific with Mallory's and Sullivan's figures of $D$. spinosa.

Dentalina spinosa d'orbigny var. ornatior Smith

Dentalina spinosa d'orbigny var. ornatior Smith, 1957,

p. 165, pl. 22, fig. 11, 12; Thoms, 1975, pl. 1, fig. 15.

Hypotype no. 00313, 10c. PSUA0104.

Dentalina substrigata [?] (Stache)

[?] Dentalina substrigata (Stache), Cushman \& Dusenbury, 1934, p. 55, pl. 7, fig. 17-19; Mallory, 1959, p. 168, pl. 13, fig. 3 .

Hypotype no. 00314 , loc. PSUA0104.

These specimens resemble $D$. substrigata in the shape of the chambers and obliqueness of the sutures but differ in possessing finer costae. 
Dentalina sp. A. [species nova]

$$
\text { (P1. 1, fig. 3, 4) }
$$

Test large, circular in cross section; chambers increasingly elongate as added; sutures indistinct, depressed; chambers ornamented with high, continuous costae, usually eight in number, extending the entire length of the test, alternating with discontinuous costae, extending to a point just below the greatest breadth of each chamber, terminated with a node forming a band around the bottom of each chamber; aperture missing.

\section{Dimensions of hypotypes :}

00315 ; length $1.02 \mathrm{~mm}$. broken; width $.26 \mathrm{~mm}$.

00316; length $.48 \mathrm{~mm}$. broken; width $.26 \mathrm{~mm}$.

Hypotype no. 00315 , loc. PSUA0131.

Hypotype no. 00316, loc. PSUA0131.

This species most closely resembles $D$. spinosa d'orbigny and $D$. spinosa d'orbigny var. ornatior Smith but differs in possessing elongate chambers and discontinuous costae which terminate in a band of nodes at the base of each chamber. It may also be synonymous with $D$. aff. $D$. spinosa of Fairchild, Wesendunk, and Weaver (1969, p. 39, description only) but lack of figures or types prevent positive identification. The naming of this species must await publication in accordance with the International code of zoological Nomenclature (1961, p. 9, art. 11a).

$$
\begin{gathered}
\text { Dentalina sp. } B \text {. } \\
\text { (Pl. 1, fig. 5) }
\end{gathered}
$$

Test large, compressed in cross section, sides parallel; chambers about equal in size, early chambers missing; sutures depressed; aperture raised, radiate, at the periphery of the terminal chamber.

Dimensions of hypotype: 00317 ; length $.46 \mathrm{~mm}$. broken; width $.21 \mathrm{~mm}$. 
Hypotype no. 00317, loc. PSUA0134.

Most samples in which this species was found, although rich in Radiolaria, were commonly lacking Foraminifera and thus were not used in this investigation. All specimens were pyritized and missing early chambers. This species resembles Dentalina sp. of Smith (1957, p. 166, pl. 22, fig. 15) and Mallory (1959, p. 168, pl. 13, fig. 8a, b) except that they lack inflated chambers.

\section{Dentalina [?] spp.}

Fragmented and poorly preserved specimens of usually not more than one chamber are included here. More than one species may be represented but accurate identification is impossible.

Genus Nodosaria Lamarck, 1812

Nodosaria arundinea Schwager

Nodosaria arundinea Schwager, Galloway \& Morrey, 1931, p. 337, pl. 37, fig. 15; Wilson, 1954, p. 136, pl. 14, fig. 12; Smith, 1956, p. 91, pl. 11, fig. 8; Mallory, 1959 , p. 169, pl. 13, fig. 10, pl. 28, fig. 7, pl. 41, fig. 4; Hornaday, 1961, p. 185, pl. 4, fig. 5; Weaver, 1962 , p. 376, pl. 2, fig. 14; Kleinpell \& Weaver, 1963, p. 171, p1. 7, fig. 4; Gaston, 1974, p. 67, [hypotype examined]; Thoms, 1975, pl. 1, fig. 21 .

Nodosaria Zongiscata d'Orbigny, Franklin, 1944, p. 311, pl. 46, fig. 1, [not fig. 2].

Nodosaria cf. N. Zongiscata d'Orbigny, Cushman, Stewart, \& Stewart, 1947 e, p. 98, pl. 13, fig. 1.

Nodosaria arundinea Schwager (?), Smith, 1957, p. 166, pl. 22, fig. 21 .

Hypotype no. 00318 , loc. PSUA0117.

Nodosaria Zongiscata d'orbigny is commonly confused with 
this species because of its elongate, slender test. $N$. Zongiscata however, has depressed sutures and shorter chambers than $N$. arundinea.

$$
\text { Nodosaria arundinea [?] Schwager }
$$

Hypotype no. 00319, 10c. PSUAO119.

Several fragmented individuals lacking sutures are questionably assigned to this species.

$$
\text { Nodosaria chirana Cushman \& Stone }
$$

Nodosaria chirana Cushman \& Stone, Cushman, Stewart, \&

Stewart, 1949, p. 131, pl. 15, fig. 1; Cushman \& Stainforth, 1951, p. 146, pl. 26, fig. 5; Mallory, 1959, p. 170, pl. 13, fig. 21; Hornaday, 1961, p. 185, pl. 4, fig. 7; Weaver \& Weaver, 1962, p. 27, pl. 7, fig. 13; Gaston, 1974, p. 67, [hypotype examined].

[Non] Nodosaria chirana Cushman \&tone, Weaver, 1962,

p. 376, pl. 2, fig. 12 .

Hypotype no. 00320 , 10c. PSUA0117.

Nodosaria deliciae Martin

Nodosaria deliciae Martin, 1943, p. 17, pl. 6, fig. 3a, b; Smith, 1957, p. 167, pl. 22, fig. 18a, b; Mallory, 1959, p. 170, pl. 13, fig. 13a, b, pl. 36, fig. 10a, b.

[?] Nodosaria deliciae Martin, Gaston, 1974, p. 68, [hypotype examined].

Hypotype no. 00321 , loc. PSUA0115.

Nodosaria cf. N. deliciae Martin

Dentalina spinosa d'Orbigny, Mallory, 1959, p. 167, pl. 12, fig. 26; Sullivan, 1962, p. 264, pl. 9, fig. 17, [not

fig. 18]; Gaston, 1974, p. 67, [hypotype examined]. Hypotype no. 00322, loc. PSUA0133.

Except for more elongate chambers, several specimens with coarse spines resemble $N$. deliciae. They appear to be 
identical with the figures of $D$. spinosa of Mallory and Sullivan. (see discussion under $D$. spinosa) Nodosaria ewaldi Reuss

Nodosaria ewaldi Reuss, Cushman \& Dusenbury, 1934, p. 56, pl. 7, fig. 21; Mallory, 1959, p. 170, pl. 13, fig. 23a, b; pl. 28 , fig. 6 .

Nodosaria ewaldi Reuss ?, Cushman, 1935, p. 22, pl. 9, fig. 2 . Hypotype no. 00323 , loc. PSUA0128.

\section{Nodosaria ewaldi [?] Reuss}

Hypotype no. 00324 , 10c. PSUA0120.

Fragmented individuals that taper toward the sutures are questionably assigned to this species.

\section{Nodosaria (?) gyrata Mallory}

Nodosaria ? gyrata Mallory, 1959, p. 170, pl. 13, fig. 18, pl. 28, fig. 5 .

[Non] Nodosaria (?) gyrata Mallory, Weaver \& Weaver, 1962, p. 27, pl. 7, fig. 14 .

Hypotype no. 00332 , loc. PSUA0104.

Nodosaria (?) gyrata Mallory [varietas nova]

$$
\text { (P1. 1, fig. 6, 7) }
$$

Variety differing from typical in the low, spiral costae which alternate with the normal higher costae.

Dimensions of hypotype:

00333 ; length $.58 \mathrm{~mm}$. broken; width $.17 \mathrm{~mm}$.

Hypotype no. $00333,10 \mathrm{c}$. PSUA0104.

The alternating fine costae easily differentiates this variety from $N$. (?) gyrata (paratypes examined). It is referred to as a variety, rather than a subspecies, since it occurs within localities with $N$. (?) gyrata. It may 
also represent local ecologic variation but until this is demonstrated, it should remain distinct. The naming of this variety must await publication in accordance with the International Code of zoological Nomenclature (1961, p. 9, art. 11a).

Nodosaria holserica Schwager

Nodosaria holserica Schwager, Kleinpell, 1938, p. 218, pl. 4, fig. 9; Sullivan, 1962, p. 265, pl. 10, fig. $2 a, b$; Fairchild, Wesendunk, \& Weaver, 1969, p. 36, pl. 4, fig. 5.

[Non] Nodosaria holserica Schwager, Smith, 1956, p. 91, pl. 10, fig. 9; Kleinpell \& Weaver, 1963, p. 171, pl. 6, fig. 9; Gaston, 1974, p. 68, [hypotype examined].

Hypotype no. 00325 , 10c. PSUA0116.

Nodosaria cf. N. holserica Schwager

Nodosaria holserica Schwager, Smith, 1956, p. 91, pl. 10, fig. 9; Kleinpell \& Weaver, 1963, p. 171, pl. 6, fig. 9. Nodosaria cf. N. holserica Schwager, Fairchild, Wesendunk, \& Weaver, 1969, p. 36, pl. 4, fig. 4, [not fig. 11].

Hypotype no. 00326, loc. PSUA0131.

Except for strongly constricted sutures, hispid individuals compare favorably with $N$. holserica.

Nodosaria latejugata Gumbel

Nodosaria Zatejugata Gumbel, Cushman \& M. A. Hanna, 1927, p. 52, pl. 5, fig. 1-3; Cushman \& McMasters, 1936, p. 512, pl. 75, fig. 11, 12; Bergquist, 1942, p. 45, pl. 4, fig. 12, 13; Kline, 1943, p. 27, pl. 2, fig. 10; Mallory, 1959 , p. 171, pl. 13, fig. 20, pl. 28, fig. 8, pl. 41, fig. 1; Weaver \& Weaver, 1962, p. 28, pl. 7, fig. 15, 16; Gaston, 1974, p. 68, [hypotype examined]; Thoms, 1975, pl. 1, fig. 22 .

Nodosaria raphanus (Linnaeus), Hanna, 1923, p. 32l, pl. 58, fig. 1. 
[Non] Nodosaria Zatejugata Gumbel, Smith, 1957, p. 167, pl. 22, fig. 23.

Hypotype no. 00327 , loc. PSUA0103.

\section{Nodosaria longiscata d'orbigny}

Nodosaria Zongiscata d'Orbigny, Kleinpell, 1938, p. 218, pl. 9, fig. 16; Howe, 1939 , p. 47, pl. 5, fig. 10;

Bergquist, 1942 , p. 46 , pl. 4, fig. 26; Kline, 1943, p. 28, pl. 2, fig. 8; Weaver \& Molander, 1964, p. 184, pl. 6, fig. 15; Todd, 1957a, pl. 65, fig. 15, 16; Phillips, Welton, \& Welton, 1976, pl. 2, fig. 7 .

[Non] Nodosaria Zongiscata d'orbigny, Franklin, 1944, p. 311, pl. 46, fig. 1, 2; Sullivan, 1962, p. 265, pl. 10, fig. 1 .

[Non] Nodosaria cf. N. Zongiscata d'orbigny, Cushman, Stewart, \& Stewart, 1947e, p. 98, pl. 13, fig. 1 .

Hypotype no. 00328 , 10c. PSUA0123.

See discussion under $N$. arundinea.

Nodosaria pyrula d'orbigny

Nodosaria pyruza d'orbigny, Cushman, 1913, p. 49, pl. 26, fig. 1-3; Cushman \& Schenck, 1928, p. 308, pl. 43, fig. 1, 2; Howe, 1939, p. $48, \mathrm{pl}$. 5, fig. 16; Wilson, 1954, p. 136, pl. 14, fig. 14; Mallory, 1959, p. 172, pl. 13, fig. 19, pl. 41, fig. 2; Hornaday, 1961, p. 185, pl. 4, fig. 6; Sullivan, 1962, p. 265, pl. 10, fig. 3a, b, $4 a, b$; Weaver \& Weaver, $1962, \mathrm{p} .28, \mathrm{pl} .7$, fig. 18; Kleinpell \& Weaver, 1963, p. 171, pl. 7, fig. 5, 6; Weaver \& Molander, 1964, p. 185, pl. 6, fig. 17; Fairchild, Wesendunk, \& Weaver, 1969, p. 37, pl. 4, fig. 12; Gaston, 1974, p. 69, [hypotype examined]; Phillips, Tipton, \& Watkins, 1974, pl. 1, fig. 7 .

Nodosaria cf. N. pyrula d'orbigny, Beck, 1943, p. 599, pl. 105, fig. 19, 20.

Dentalina pyrula (d'Orbigny), Puri, 1953, p. 100, pl. 23, fig. $12,13$.

Hypotype no. 00329, loc. PSUA0107. 
Nodosaria pyrula [?] d'orbigny

Hypotype no. 00330 , 10c. PSUA0112.

Bulbous, single chambered specimens seem best referred to this species.

Nodosaria sp. [of Mallory]

Nodosaria sp. Mallory, 1959, p. 173, pl. 13, fig. 17.

Hypotype no. 00331 , loc. PSUA0137.

A costate fragment from the Salmon River section appears identical to the specimen figured by Mallory.

$$
\text { Nodosaria [?] spp. }
$$

Accurate identification of numerous fragmented, single chambered specimens is impossible. More than one species is probably represented.

Genus Pseudoglandulina Cushman, 1929

Pseudoglandulina conica (Neugeboren)

Pseudoglandulina conica (Neugeboren), Cushman \& Barksdale, 1930 , p. 65 , pl. 12, fig. 1-3; Beck, 1943, p. 599, pl. 105, fig. 12; Smith, 1957, p. 168, pl. 23, fig. la, b, 2; Mallory, 1959, p. 173, pl. 33, fig. 4, pl. 36, fig. lla, b; Weaver \& Weaver, $1962, \mathrm{p} .28, \mathrm{pl} .8$, fig. 1, 2; Weaver \& Molander, 1964, p. 185, pl. 6, fig. 18, pl. 7, fig. 1; Gaston, 1974, p. 69, [hypotype examined].

Hypotype no. 00334 , 10c. PSUA0103.

Pseudoglandulina ovata (Cushman \& Applin)

Nodosaria (Glandulina) laevigata d'orbigny var. ovata Cushman \& Applin, 1926, p. 26, pl. 7, fig. 12, 13.

GLandulina Zaevigata d'Orbigny var. ovata (Cushman \& Applin), Cushman, 1935, p. 29, pl. 10, fig. 16, 17; Cushman, Stewart, \& Stewart, 1947d, p. 76, pl. 9, fig. 7; Smith, 1956, p. 92, pl. 1l, fig. 1la, b. 
Pseudoglandulina ovata (Cushman \& Applin), Mallory, 1959, p. 174 , pl. 28, fig. 9a, b; Sullivan, 1962, p. 266, pl. 11, fig. 2a, b, 3, 4; Kleinpell \& Weaver, 1963, p. 171, pl. 7,

fig. 7a, b; Weaver \& Molander, 1964, p. 185, pl. 7,

fig. 2; Gaston, 1974, p. 70, [hypotype examined].

Hypotype no. $00335,10 c$. PSUA0110.

\section{Genus Saracenaria Defrance, 1824}

Saracenaria cf. S. moresiana Howe \& Wallace

Hypotype no. 00336, loc. PSUA0132.

Except for a keel along the outer margin of the test, a single specimen from the Salmon River Section is identical with specimens of $S$. moresiana as figured by Bergquist (1942, p. 48, p1. 3, fig. 14a, b), Weaver and Weaver $(1962$, p. 28, pl. 8, fig. 5), and Weaver and Molander (1964, p. 185, pl. 7, fig. 7).

\section{Saracenaria sp.}

Hypotype no. 00337, loc. PSUA0113.

A few individuals from the Neskowin Creek Section seem best referred to this genus.

\section{Genus Lagena Walker \& Jacob, 1798 \\ Lagena acuticosta Reuss}

[Non] Lagena acuticosta Reuss, Bagg, 1905, p. 45, pl. 14, fig. 28; Bergquist, 1942 , p. 49, pl. 5, fig. 30 .

Lagena acuticosta Reuss, Cushman, 1913, p. 23, pl. 8, fig. 9a, b, 10, pl. 23, fig. 2; Cushman, 1935, p. 23, pl. 9, fig. 5, 6; Kleinpell, 1938, p. 224 , pl. 7, fig. 13; Cushman, $1946 \mathrm{a}$, p. 94 , pl. 39 , fig. 14,15 ; Cushman \& Stainforth, 1951, pl 148, pl. 26, fig. 10; Frizzell, 1954, p. 102, pl. 14, fig. 1, 2; Puri, 1957, p. 115, pl. 4, fig. 9a, b; Mallory, 1959, p. 174, pl. 14, fig. la, b, pl. 28, fig. 10a, b, pl. 4l, fig. 8a, $b$; Weaver \& Molander, 1964 , p. 186, pl. 7, fig. 10; Gaston, 1974, p. 71, [hypotype examined]; Murray \& Wright, 1974, pl. 6, fig. 2. 
Lagena conscripta Cushman Barksdale, Beck, 1943, p. 601, pl. 107, fig. 38; Sullivan, 1962, p. 66, pl. 10,

fig. $10 a, b$.

Hypotype no. 00338 , 10c. PSUA0133.

Lagena conscripta Cushman and Barksdale has commonly been confused with $L$, acuticosta in West Coast literature. The apertural end of $L$. conscripta, as described by Cushman and Barksdale (1930, p. 66), has a "definite expanded area appearing almost like a secondary chamber" which indeed is very similar to the apertural end of $L$. acuticosta. They further state however, that the expanded area is "apparently due entirely to ornamentation which consists of several, 16-20, longitudinal costae". These costae which make up the "secondary chamber" are "increased slightly in number and not entirely confluent with those of the main body of the test". Of all of the figures examined for this investigation, none possessed an expanded apertural region composed of longitudinal costae. Therefore, these forms have been synonymized with $L$. acuticosta.

\section{Lagena amphora Reuss}

Lagena costata (Williamson) var. amphora Reuss, Cushman, 1913, p. 21, pl. 10, fig. 2, 3, pl. 12, fig. 2; Howe, 1939, p. 49, pl. 6, fig. 15; Puri, 1953, p. 102, pl. 21, fig. 6 .

Lagena amphora Reuss, Mallory, 1959, p. 175, pl. 14, fig. $2 a, b$.

Hypotype no. 00339 , 10c. PSUA0133.

Lagena becki sullivan

Lagena sp. A. Beck, 1943, p. 602, pl. 107, fig. 26. Lagena sp. Smith, 1956, p. 92, pl. 10, fig. $4 a, b$. 
Lagena becki Sullivan, 1962, p. 266, pl. 10, fig. 16a, b; Fairchild, Wesendunk, \& Weaver, 1969, p. 39, pl. 5, fig. 10; Smith, 1971, p. 39, p1. 5, fig. 3.

Hypotype no. 00340 , 10c. PSUA0118.

Lagena costata (Williamson)

Lagena costata (Williamson), Cushman, 1913, p. 21, pl. 9, fig. 6, pl. 10, fig. 1, pl. 12, fig. 1; Cushman, 1935, p. 23, pl. 9, fig. 7, 8; Coryell \& Embich, 1937, p. 299, pl. 42, fig. 11; Beck, 1943, p. 601, pl. 107, fig. 36; Franklin, 1944, p. 313, pl. 45, fig. 17, Mallory, 1959, p. 175, pl. 14, fig. 3a, b, pl. 4l, fig. 7a, b; Gaston, 1974, p. 71, [hypotype examined].

Lagena costata (?) (Williamson), Bergquist, 1942, p. 49, pl. 5, fig. 23.

Hypotype no. 00341 , loc. PSUA0125.

This species, as figured by various authors, has a highly variable number of costae. It most closely resembles $L$. strumosa Reuss (Sullivan, 1962, p. 267, pl, 10, fig. 9a, b) but differs recognizably in having fewer costae, a long apertural neck, and does not possess a basal spine.

$$
\text { Lagena costata [?] (Williamson) }
$$

Hypotype no. 00342 , loc. PSUA0133.

Poorly preserved specimens are referred to this species. Lagena hexagona (Williamson)

Lagena hexagona (Williamson), Cushman, 1913, p. 17, pl. 6, fig. 2, 3; Cushman, 1931, p. 38, pl. 5, fig. 12; Cushman, 1935 , p. 23, pl. 9, fig. 10; Howe, 1939, p. 50 , pl. 6, fig. 16; Bergquist, 1942, p. 50, pl. 5, fig. 24; Beck, 1943, p. 602, pl. 107, fig. 23; Cushman, 1946a, p. 95, pl. 39, fig. 16; Mallory, 1959, p. 175, pl. 14, fig. 7; sullivan, 1962, p. 267, pl. 10, fig. 9a, b; Weaver \& Molander, 1964, p. 187, pl. 7, fig. 13; Fairchild, Wesendunk, \& Weaver, 1969 , p. 40, pl. 5, fig. 13; Gaston, 1974, p. 71, [hypotype examined]; Philiips, Tipton, \& Watkins, 1974, pl. 1 , fig. 5 . 
Entosolenia hexagona Williamson, Cushman \& Simonson, 1944, p. 198, pl. 32, fig. 8 .

Hypotype no. 00343 , loc. PSUA0137.

Lagena marginata (Walker \& Boys)

Lagena marginata (Walker \& Boys), Bagg, 1905, p. 27, pl. 4, fig. 5; Cushman, 1913 , p. 37, pl. 22, fig. 1-7; Kleinpell, 1938 , p. 225 , pl. 10 , fig. 5; Smith, 1956, p. 92, pl. 10, fig. la, b; Kleinpell \& Weaver, 1963, p. 172, pl. 7, fig. 12; Fairchild, Wesendunk, \& Weaver, 1969, p. 40, p1. 5, fig. 14; Gaston, 1974, p. 72, [hypotype examined].

[Non] Lagena marginata (Walker \& Boys), Bagg, 1912, p. 49, pl. 14, fig. 18a-c, $21 a, b, 22 a, b$.

Entosolenia marginata (Walker \& Boys), Bergquist, 1942, p. 67, pl. 7, fig. 10 .

Fissurina marginata (Walker \& Boys), Murray \& Wright, 1974, pl. 6, fig. 5 .

Hypotype no. 00344 , loc. PSUA0101.

Lagena vulgaris Williamson

Lagena vulgaris Williamson, Cushman, 1946a, p. 95, pl. 40 , fig. 3; Mallory, 1959, p. 176, pl. 14, fig. 9a, b; Sullivan, 1962, p. 267, pl. 10, fig. 12a, b, 13; Weaver \& Molander, 1964 , p. 187, pl. 7, fig. 14; Gaston, 1974, p. 72,

[hypotype examined].

Hypotype no. 00345, loc. PSUA0112.

Family POLYMORPHINIDAE

Genus Guttulina d'Orbigny, 1839

Guttuzina frankei Cushman \& Ozawa

Guttulina frankei Cushman \& Ozawa, Cushman \& Frizzell, 1943, p. 84 , pl. 14, fig. 17, 18; Rau, 1948, p. 170, pl. 30, fig. 17,18 .

Hypotype no. 00346, loc. PSUA0102.

A single recrystallized specimens was recovered from the basal Neskowin Creek Section. Its greater size and somewhat 
different chamber arrangement differentiate this species from Guttulina irregularis d'orbigny. Guttulina irregularis (d'orbigny)

Polymorphina byramensis Cushman, Cushman, 1923, p. 31, pl. 5, fig. $1-5$.

Guttulina byramensis (Cushman), Cushman \& Schenck, 1928, p. 309, pl. 43, fig. 6-8; Detling, 1946, p. 354, pl. 48, fig. 10 .

Guttulina irregularis (d'orbigny), Cushman, 1935, p. 24, pl. 9, fig. 13-16; Bergquist, 1942, p. 54, pl. 6, fig. 5, [not fig. 6]; Beck, 1943, p. 602, pl. 106, fig. 3, 15; Cushman \& Simonson, 1944, p. 196, pl. 31, fig. 10-12; Rau, 1948 , p. 169 , pl. 30, fig. 7, 8; Puri, 1957, p. 116, pl. 5, fig. 10a-c; Sullivan, 1962, p. 267 , pl. 1l, fig. 8a-c; Gaston, 1974, p. 72, [hypotype examined].

[Non] Guttlina irregularis (d'Orbigny), Howe, 1939, p. 52, pl. 6, fig. 20; Puri, 1953, p. 107, pl. 9, fig. 8, 9, 10; Asano, 1958, pl. 10, fig. 17; Mallory, 1959, p. 177, pl. 14, fig. 13a, b.

Guttulina cf. G. irregularis (d'Orbigny), Kleinpell, 1938, p. 228, pl. 2, fig. 7 .

Guttulina sp. Wilson, 1954, p. 137, pl. 15, fig. 4a, b. Guttulina problema d'orbigny, Kleinpell \& Weaver, 1963, p. 172, pl. 2, fig. 13 . Hypotype no. 00347 , loc. PSUA0128. The difficulty in separating $G$. irregularis from $G$. problema d'orbigny has given rise to much confusion in the published literature. Guttulina irregularis, as figured by many authors, is identical to other forms referred to as $G$. problema. Some authors have even synonymized the two (Smith, 1956, p. 92; Kleinpell and Weaver, 1963, p. 172). Individuals referred to as $G$. irregularis in this investigation are small, triangular in outline, and consist of one or two early 
chambers overlapped by two later chambers which usually extend back to the proloculus. Guttulina problema is similar except for its larger size, greater number of chambers, and later chambers which do not extend back to the proloculus but are added at higher levels. It is quite possible that G. problema represents a mature individual; G. irregularis being a juvenile. Further study of an ontogenetic series may show that $G$. irregularis and $G$. problema are synonymous; until this is accomplished, these forms remain separated.

$$
\text { Guttulina [?] sp. }
$$

Hypotype no. 00348 , 10c. PSUA0107.

A single specimen, questionably assigned to this genus, has early overlapping chambers terminated by a single uniserial chamber.

\section{Genus Ramulina Rupert Jones, 1875 \\ Ramulina aff. R. navarroana Cushman [of Mallory]}

Ramulina aff. R. navarroana Cushman, Mallory, 1959, p. 178, pl. 33, fig. 5 .

Hypotype no. 00349 , 10c. PSUA0112.

A single specimen from the Neskowin Creek Section compares well with the specimen figured by Mallory.

Family NONIONIDAE

Genus Nonion Montfort, 1808

Nonion durhami Mallory

Nonion havanense Cushman \& Bermudez (?), Smith, 1957, p. 171, pl. 23, fig. $17 a, b$. 
Nonion durhami Mallory, 1959, p. 181, pl. 15, fig. 4a, b, pl. 28, fig. 1la, b; Smith, 1971, p. 64, pl. 5, fig. 6a, b. Hypotype no. 00350 , loc. PSUA0106.

Specimens from the Neskowin Creek Section have radiate sutures, nine chambers in the last whorl, and compare favorably with Smith's (1957) and Mallory's figures. The specimen figured by Smith (1971) has somewhat limbate sutures and only seven chambers in the last whorl and may not represent this species.

\section{Nonion durhami [?] Mallory}

Hypotype no. 00351, loc. PSUA0103.

A single recrystallized specimen from the basal Neskowin Creek section seems referable to this species. Nonion planatum Cushman \& Thomas

Nonion planatum Cushman \& Thomas, Cushman \& Dusenbury, 1934, p. 60, pl. 8, fig. $6 \mathrm{a}, \mathrm{b}$; Cushman, 1939 , p. 4, pl. 1 , fig. $15 a, b$; Howe, 1939 , p. 58 , pl. 7 , fig. 24, 25; Bergquist, 1942 , p. 61, pl. 6, fig. 24; Beck, 1943, p. 603, pl. 107, fig. 12, 13; Bandy, 1944, p. 370 , pl. 60 , fig. 15a-c; Cushman \& Stainforth, 1951, p. 149, pl. 26, fig. 18; Todd, 1957a, pl. 65, fig. 28a, b; Mallory, 1959, p. 181, pl. 15, fig. $2 a, b$; Weaver \& Weaver, 1962, p. 29, pl. 8, fig. 7a, b; Kleinpell \& Weaver, 1963, p. 173, pl. 8, fig. 3a, b; Gaston, 1974, p. 73, [hypotype examined]; Thoms, 1975, pl. 2, fig. 9a, b.

Hypotype no. 00352 , loc. PSUA0125.

This widely recorded species is easily recognized by slightly curved sutures which terminate in a thickened ring around the umbilici. Although most published figures of $N$. planatum depict a close coiled form with a rounded periphery, many specimens from the Neskowin Creek Section have a lobate outline. The amount of lobation varies; individuals with 
completely lobate outlines grade into forms in which only the last one or two chambers are lobate. According to Rau (personal communication, 1976) this difference falls within the range of variation of $N$. planatum and thus does not warrant the proposal of a new specific name. Nonion planatum [?] Cushman \& Thomas Hypotype no. $00353,10 c$. PSUA0131.

A few crushed specimens probably represent this species. Nonion sp. A. [species nova]

$$
\text { (P1. 1, fig. 8, 9) }
$$

Test longer than broad, compressed, bilaterally symmetrical, umbilical area slightly depressed, periphery subacute to rounded; chambers distinct, eight in number in the adult coil, increasing rapidly in size as added, slightly inflated; sutures distinct, slightly curved, moderately depressed; wall smooth; aperture indistinct, at the base of the last formed chamber.

Dimensions of hypotype: 00354; length . $31 \mathrm{~mm}$.; breadth $.24 \mathrm{~mm}$. ; thickness $.18 \mathrm{~mm}$. Hypotype no. 00354 , loc. PSUA0119.

This species most closely resembles Nonion mexicanum Cole (Cushman, 1939, p. 6, pl. 1, fig. 23a, b, 24a, b) but differs from it in possessing an additional chamber in the final whorl, greater curvature of the sutures and larger, more inflated chambers. Specimens identical to $N$. sp. A. except for acute peripheries are probably the result of compaction and thus do not warrant a separate specific designation. The naming of this species must await publication in accordance with the International Code of zoological Nomeclature (1961, p. 9, art. 11a). 
Nonion sp.

Hypotype no. 00355 , loc. PSUA0137.

Poorly preserved individuals are only identifiable at a generic level.

\author{
Genus Elphidium Montfort, 1808 \\ Elphidium smithi Cushman \& Dusenbury
}

Elphidium smithi Cushman \& Dusenbury, 1934, p. 61, pl.8,

fig. $7 a, b$; Cushman, 1939, p. 40, pl. 10, fig. 19a, b.

Hypotype no. 00356 , 10c. PSUA0102.

A single specimen from the basal Neskowin Creek Section, although recrystallized, has numerous chambers, is of large size, and possesses the double row of pores which characterize this species.

$$
\begin{gathered}
\text { Elphidium sp. A. [species nova] } \\
\text { (P1. 1, fig. } 10,11 \text { ) }
\end{gathered}
$$

Test small, longer than broad, compressed, bilaterally symmetrical, umbilical area slightly depressed, filled with numerous papillae which extend onto the base of the apertural face and early chambers, periphery subacute to rounded; early chambers obscured by papillae, later chambers distinct, increasing in size as added, slightly inflated, seven to eight in number; sutures depressed, retral processes distinct, appearing as a line of pores; wall smooth; aperture obscured by papillae.

Dimensions of hypotype:

00357 ; length .26 mm.; breadth $.18 \mathrm{~mm}$; thickness $.13 \mathrm{~mm}$.

Hypotype no. 00357, 10c. PSUA0101.

This species somewhat resembles the general outline of Elphidium texanum (Cushman and Applin) (Cushman, 1939, p. 39, pl. 10, fig. 17a, b) but the numerous papillae in the umbilical area and on the early chambers easily differentiates the 
two. Several specimens with acute peripheries are included under this designation as they probably represent compressed individuals. The naming of this species must await publication in accordance with the International Code of Zoological Nomenclature (1961, p. 9, art. 1la). Elphidium sp.

Hypotype no. 00358 , loc. PSUA0127.

A few specimens resembling Elphidium sp. A. possess very weathered tests making specific identification impossible. Family HETEROHELICIDAE

Genus Plectofrondicularia Liebus, 1903 Plectofrondicularia gracilis Smith Plectofrondicularia gracilis Smith, 1956, p. 93, pl. 12, fig. 2-5; Kleinpell \& Weaver, 1963, p. 174, pl. 8, fig. 11, [not fig. 8]; Fairchild, Wesendunk, \& Weaver, 1969 , p. 47, pl. 10, fig. 11.

[?] Plectofrondicularia gracilis Smith, Asano, 1958, text-fig. 5,6 .

Hypotype no. 00359 , loc. PSUA0128.

This species intergrades with Plectofrondicularia packardi Cushman and Schenck var. multilineata Cushman and Simonson (Smith, 1956, p. 93) and in some cases the two are impossible to separate. Specimens assigned to this species from the Neskowin Creek Section differ from $P$. packardi var. multilineata in possessing a narrower test, more angular sutures, and six strong costae that alternate with a few finer costae. 
Plectofrondicularia kerni cook

Plectofrondicularia kerni Cook, Mallory, 1959, p. 212, pl. 18, fig. 2, pl. 33, fig. 10a, b; sullivan, 1962, p. 269, pl. 13, fig. 7, 8, [not fig. 6]; Fairchild, Wesendunk, \& Weaver, 1969, p. 47, pl. 10, fig. 4.

Hypotype no. 00360 , loc. PSUA0116.

This species, as figured in the published literature, exhibits a large amount of variation in the number of longitudinal costae it possesses. According to cook (in Mallory, 1959, p. 212), Plectofrondicularia kerni has 35 to 40 low, distinct costae whereas other published figures examined during this investigation depict a form with considerably fewer costae (5 to 17). A single specimen assigned to this species from the Neskowin Creek Section possesses ten longitudinal costae and all other respects is identical to the specimens figured by Mallory.

\section{Plectofrondicularia oregonensis Cushman, Stewart, \& Stewart}

Plectofrondicularia oregonensis Cushman, Stewart, \& Stewart, $1947 \mathrm{e}, \mathrm{p} .100$, pl. 13, fig. 8a, b, 9a, b; Cushman, Stewart, \& Stewart, $1947 \mathrm{~d}, \mathrm{p} .77, \mathrm{pl}$. 10, fig. 4a, b; Fairchild, Wesendunk, Weaver, 1969, p. 48, pl. 9, fig. 6; Smith, 1971, p. 47, pl. 5, fig. 7 .

[?] PZectofrondicularia searsi Cushman, Stewart, \& Stewart, Mallory, 1959, p. 214, pl. 17, fig. 17.

[?] PlectofrondicuZaria packardi packardi Cushman \& Schenck, Rau, 1964, p. Gl7, pl. 5, fig. 13.

Hypotype no. 00361, loc. PSUA0128.

This species, although similar to Plectofrondicularia

searsi Cushman, Stewart, and Stewart (Sullivan, 1962, p. 270, 
p1. 13, fig. 9), is distingished by its small size and two strong median costae.

Plectofrondicularia packardi Cushman \& Schenck var. multilineata Cushman \& Simonson

Plectofrondicularia packardi Cushman \& Schenck var. multilineata Cushman \& Simonson, 1944, p. 197, pl. 32, fig. 2-4; Cushman \& Stainforth, 1951, p. 150, pl. 26, fig. 26; Wilson, 1954, p. 138, pl. 15, fig. 9; Hornaday, 1961 , p. 187, pl. 4, fig. 21; Sullivan, 1962, p. 270, pl. 12, fig. 10; Weaver \& Molander, 1964, p. 187, pl. 8, fig. 3; Delise, 1967, p. 33, pl. 2, fig. 14; Fairchild, Wesendunk, \& Weaver, 1969, p. 48, pl. 9, fig. 16; Tipton, Kleinpell, \& Weaver, 1973, p. 51, pl. 4, fig. 9; Phillips, Tipton, \& Watkins, 1974, pl. 1, fig. 14; Phillips, Welton, \& Welton, 1976, pl. 3, fig. 8 .

Plectofrondicularia packardi multilineata Cushman \& Simonson, Detling, 1946 , p. 355, pl. 49, fig. 3, 5; Rau, 1948, p. 171, pl. 30, fig. 19; Asano, 1952, p. 40, pl. 4, fig. 4, pl. 5, fig. 3-5; Smith, 1956, p. 94, pl. 12, fig. 6; Asano, 1958, text-fig. 3; Smith, 1971, p. 48, pl. 6, fig. 1.

Plectofrondicularia packardi Cushman \& Schenck var. multi-lineata Cushman \& Simonson, Weaver \& Weaver, 1962, p. 30, pl. 8, fig. 11 .

[Non] PZectofrondicularia packardi multilineata Cushman \& Simonson, Rau, 1964, p. Gl6, pl. 5, fig. 15.

Hypotype no. 00362 , loc. PSUA0131.

This variety, as figured by Cushman and simonson (1944, pl. 32, fig. 2-4), exhibits some variation in the length of its longitudinal costae; in figure 4 the costae extend the entire length of the test while in figure 2 and 3 the costae are restricted to the lower one-half of the test. Specimens from the "Strata of Cascade Head" normally possess costae which extend the entire length of the test. 
Plectofrondicularia packardi Cushman \& Schenck var. packardi Cushman \& Schenck

Plectofrondicularia packardi Cushman \& Schenck, 1928, p. 311, pl. 43, fig. 14, 15; Cushman \& Simonson, 1944, p. 197, pl. 31, fig. 17, 18, pl. 32, fig. 1; Detling, 1946, p. 355, p1. 49, fig. 1; Asano, 1952, p. 39, pl. 5, fig. 1, 2; Wilson, 1954, p. 138, pl. 15, fig. 8; Asano, 1958, text-fig. 1, 2; Hornaday, 1961, p. 187, pl. 4, fig. 20.

[Non] PZectofrondicularia packardi Cushman \& Schenck, Cushman, Stewart, \& Stewart, 1947 e, p. 100, pl. 13, fig. $7 a, b$.

Plectofrondicularia packardi packardi Cushman \& Schenck, Smith, 1956, p. 94, pl. 12, fig. 1, 7; Sullivan, 1962, p. 270, pl. 12, fig. 11, 12; Phillips, Tipton, \& Watkins, 1974, pl. 1, fig. 10, 11, [not fig. 12].

[?] PZectofrondicularia packardi Cushman \& Schenck var. packardi Cushman \& Schenck, Mallory, 1959, p. 213, pl. 17, fig. 16.

Plectofrondicularia packardi Cushman \& Schenck var. packardi Cushman \& Schenck, Kleinpell \& Weaver, 1963, p. 174, pl. 8, fig. 5-7, 10, 14; Weaver \& Molander, 1964 , p. 188, pl. 8, fig. 4; Fairchild, Wesendunk, \& Weaver, 1969, p. 48 , pl. 9, fig. 20; Tipton, Kleinpell, \& Weaver, 1973, p. 50, pl. 4, fig. 8 .

[Non] PZectofrondicularia packardi packardi Cushman \& Schenck, Rau, 1964, p. Gl7, pl. 5, fig. 13.

Hypotype no. $00363,10 c$. PSUA0128.

This widely recorded variety is characterized by a large, wide test, rounded sutures, and short costae on the early portion of the test.

Plectofrondicularia aff. P. packardi Cushman \& Schenck var. packardi Cushman \& Schenck

Plectofrondicularia packardi Cushman \& Schenck, Cushman, Stewart, \& Stewart, 1947e, p. 100, pl. 13, fig. 7a, b.

Plectofrondicularia packardi packardi Cushman \& Schenck, Rau, 1964 , p. G17, pl. 5, fig. 13. 
[?] Plectofrondicularia packardi packardi Cushman \& Schenck, Phillips, Tipton, Watkins, 1974, pl. 1, fig. 12, [not fig. 10,11$]$.

Hypotype no. 00364 , 10c. PSUA0131.

Individuals assigned to this variety are characterized by a large, slightly keeled test that increases rapidly in size as chambers are added, acutely angular sutures, and costae which cover the proloculus and extend to various lengths above the early portions of the test. It differs from $P$. packardi var. packardi sensu stricto in possessing acutely angulate sutures and from $P$. searsi Cushman, Stewart, and Stewart in possessing a much wider test.

This variety is most closely related to $P$. packardi var. packardi s. s. and in some instances, it is very difficult to differentiate the two. Plectofrondicularia aff. $P$. packardi var. packardi is restricted to the upper portions of the Neskowin Creek section in which it occurs commonly with both $P$. searsi and $P$. packardi var. packardi s. $s$. Since it possesses characteristics of both, and intergrades with $P$. packardi var. packardi s. s., specimens referred to this variety probably represent an intermediate form.

Plectofrondicularia searsi Cushman, Stewart, \& Stewart

Plectofrondicularia searsi Cushman, Stewart, \& Stewart, 1947d, p. 78, pl. 10, fig. 5a, b, pl. 11, fig. 8a, b; Cushman, Stewart, \& Stewart, 1949, p. 132, pl. 15, fig. 5a, b; Sullivan, 1962 , p. 270, pl. 13, fig. 9; Delise, 1967, p. 33, pl. 3, fig. 1; Smith, 1971, p. 48, pl. 6, fig. 2; Gaston, 1974, p. 75, [hypotype examined]. 
[Non] Plectofrondicularia searsi Cushman, Stewart, \& Stewart, Mallory, 1959, p. 214, pl. 17, fig. 17.

Hypotype no. 00365, loc. PSUA0130.

See discussion under $P$. oregonensis and $P$. aff. $P$. packardi var. packardi.

\section{Plectofrondicularia vaughni Cushman}

Plectofrondicularia vaughni Cushman, Coryell \& Embich, 1937,

p. 303, pl. 42, fig. 15; Cushman \& Stainforth, 1951,

p. 150, pl. 26, fig. 27; Smith, 1956, p. 94, pl. 11,

fig. 14; Sullivan, 1962, p. 271, pl. 12, fig. 7; Kleinpell \& Weaver, 1963, p. 174, p1. 8, fig. 12; Weaver \& Molander, 1964 , p. 188, pl. 8, fig. 6; Fairchild, Wesendunk, \& Weaver, 1969, p. 48, pl. 9, fig. 10; Smith, 1971, p. 48, pl. 6, fig. 3; Tipton, Kleinpell, \& Weaver, 1973, p. 51, pl. 4, fig. 1l; Phillips, Welton, \&elton, 1974, pl. 3, fig. 9 .

Hypotype no. 00366 , 10c. PSUA0116.

Specimens with alternating early chambers appear identical to this widely recorded species.

\section{Plectofrondicularia vokesi Cushman, Stewart, \& Stewart}

Plectofrondicularia vokesi Cushman, Stewart, \& Stewart, 1949, p. $132, \mathrm{pl}$. 15, fig. 4; Mallory, 1959, p. $214, \mathrm{pl} .17$, fig. 18; Sullivan, 1962, p. 271, pl. 13, fig. 10; Weaver \& Weaver, 1962, p. 30, pl. 8, fig. 16; Kleinpell \& Weaver, 1963 , p. 174, pl. 9, fig. 2; Weaver \& Molander, 1964, p. 188, pl. 8, fig. 7; Delise, 1967, p. 33, pl. 3, fig. 3; Fairchild, Wesendunk, \& Weaver, 1969, p. 49, pl. 9, fig. 7.

Plectofrondicularia cf. P. vokesi Cushman, Stewart, \& Stewart, Phillips, Tipton, \& Watkins, 1974, pl. 1, fig. 13.

[Non] PZectofrondicularia vokesi Cushman, Stewart, \& Stewart, Thoms, 1975, pl. 2, fig. 12 .

Hypotype no. $00367,10 c$. PSUA0118.

Plectofrondicularia sp. [of Weaver \& Weaver]

Plectofrondicularia sp. Weaver \& Weaver, 1962, p. 30, pl. 8, fig. 16. 
Hypotype no. 00368 , 10c. PSUA0121.

This species resembles Plectofrondicularia minuta sullivan (1962, p. 269, pl. 13, fig. 1-4) but has fewer chambers in the biserial stage and more angular sutures.

$$
\text { Plectofrondicularia sp. }
$$

Hypotype no. 00369 , loc. PSUA0124.

Several fragments can only be identified to a generic level. Genus Amphimorphina Neugeboren, 1850 Amphimorphina jenkinsi (Church)

Plectofrondicularia jenkinsi Church, 1931, pl. A, fig. 5, 7-9; Detling, 1946, p. 355, pl. 49, fig. 2a, b, [? fig. 8].

Amphimorphina jenkinsi (Church), Mallory, 1959, p. 216, pl. 18, fig. 5; Weaver \& Weaver, 1962, p. 31, pl. 9, fig. 2; Kleinpell \& Weaver, 1963, p. 174, pl. 9, fig. 3; Gaston, 1974, p. 75, [hypotype examined].

[?] Amphimorphina jenkinsi (Church), Sullivan, 1962, p. 272, pl. 13, fig. 15.

Hypotype no. 00370 , loc. PSUA0119.

Genus Nodogenerina Cushman, 1927

Nodogenerina adolphina (d'orbigny)

[Non] Nodosaria adolphina (d'Orbigny), Bagg, 1905, p. 28, pl. 5, fig. 1 .

[?] Dentalina adolphina d'orbigny, Cushman \& Schenck, 1928, p. 308 , pl. 42 , fig. 6 .

Dentalina adolphina d'orbigny, Bergquist, 1942, p. 40, p1. 5, fig. 1.

Nodogenerina adolphina (d'orbigny), Mallory, 1959, p. 216, pl. 18, fig. 8a, b, pl. 29, fig. 8a, b, pl. 41, fig. 10; Sullivan, 1962, p. 272, pl. 13, fig. 17a, b; Weaver \& Molander, 1964, p. 188, pl. 8, fig. 8; Gaston, 1974, p. 75, [hypotype examined].

Hypotype no. 00371, loc. PSUA0116. 
Nodogenerina adolphina, as figured by various authors, occurs in two forms; a form with globular chambers separated by constricted sutures (Mallory, 1959, pl. 18, fig. 8a, b, pl. 29, fig. 8a, b) and a form that has a uniform diameter throughout its length except for the final chambers (Mallory, 1959, p1. 41, fig. 10; Weaver and Molander, 1964, pl. 8, fig. 8). Both forms are represented in the "Strata of Cascade Head" and are included under this designation.

Individuals assigned to this species are similar to $N$. lepidula Schwager (Weaver and Weaver, 1962, pl. 8, fig. 9) in that they both possess a row of spines around the lower margins of each chamber. Nodogenerina adolphina however, possesses a regular pattern of spines on the chambers and less constricted sutures whereas, $N$. Zepidula has a row of irregularly spaced spines and extremely constricted, limbate sutures.

Nodogenerina cf. N. bradyi Cushman

kodogenerina bradyi Cushman, Mallory, 1959, p. 216, pl. 18, fig. 12 .

Hypotype no. 00372 , 10c. PSUA0123.

Although the specimen figured by Mallory is similar to $N$. bradyi, it does not agree with Cushman's type description (1927, p. 79, no figure) or other published figures (Church, 1931, pl. C, fig. 6, 7; Detling, 1946, pl. 49, fig. 9, 10; Cushman, 1951, pl. 26, fig. 3, 7). Nodogenerina brudyi possesses fine spinose projections at the base of each chamber whereas the form figured by Mallory lacks these spinose 
projections and is smaller in size. Specimens from the "Strata of Cascade Head" which appear identical to the form figured by Mallory have therefore been designated $N$. cf. $N$. bradyi.

Nodogenerina kressenbergensis (Gumbel)

Nodosaria kressenbergensis Gumbel, Weinzierl \& Applin, 1929, p. 398, p1. 43, fig. 2 .

Nodogenerina kressenbergensis (Gumbel) (?), Smith, 1957, p. 172, pl. 24, fig. 5 .

Nodogenerina kressenbergensis (Gumbel), Mallory, 1959,

p. 217, pl. 18, fig. 11, pl. 40, fig. 13.

Hypotype no. 00373 , loc. PSUA0133.

This species is characterized by a small, delicate test and chambers joined to each other by long slender necks. One of Mallory's figures (1959, pl. 40, fig. 13) has costae around the central portion of each chamber and thus may represent another species.

Nodogenerina lepidula (Schwager)

Nodosaria Zepidula Schwager, Galloway \& Morrey, 1931, p. 337, pl. 38, fig. 1.

Nodogenerina Zepidula (Schwager), Mallory, 1959, p. 217, pl. 18, fig. 10; Weaver \& Weaver, 1962, p. 29, pl. 8, fig. 9; Weaver \& Molander, 1964, p. 189, pl. 8, fig. 11; Gaston, 1974, p. 76, [hypotype examined]; Thoms, 1975, pl. 1, fig. 20 .

Hypotype no. 00374 , loc. PSUA0131.

See discussion under $N$. adolphina.

$$
\text { Nodogenerina lepidula [?] (Schwager) }
$$

Hypotype no. 00375, loc. PSUA0107.

Poorly preserved specimens that resemble $N$. Zepidula in outline but lack surface ornamentation are included here. 
Nodogenerina spp.

Hypotype no. 00376, loc. PSUA0133.

Specimens consisting of single chambers and fragments are questionably referred to this genus. More than one species may be represented.

\section{Family BULIMINIDAE}

Genus Bulimina d'orbigny, 1826

Bulimina consanguinea Parker \& Bermudez

Bulimina consanguinea Parker \& Bermudez, Cushman \& Parker, 1946 , p. 99, pl. 23, fig. 5a-c; Mallory, 1959, p. 189, pl. 16, fig. 6 .

Hypotype no. 00377 , 10c. PSUA0105.

\section{Bulimina corrugata Cushman \& Siegfus}

Bulimina corrugata Cushman \& Siegfus, 1935, p. 92, pl. 14, fig. $7 a, b$; Kleinpell, 1938, p. 252, pl. 4, fig. 13; Cushman \& Parker, 1946, p. 93, pl. 22, fig. 2a, b; Smith, 1957, p. $174, \mathrm{pl}$. 24 , fig. 8a, b; Asano, 1958, pl. 12, fig. 15; Mallory, 1959, p. 189, pl. 28, fig. 13a, b; Weaver, 1962, p. 378, pl. 3, fig. 5a-c; Weaver \& Weaver, 1962, p. 31, pl. 9, fig. 3; Kleinpell \& Weaver, 1963, p. 175, pl. 9, fig. 12; Rau, 1964, p. G17, pl. 5, fig. 11; Fairchild, Wesendunk, \& Weaver, 1969, p. 53, pl. 11, fig. 5; Tipton, Kleinpell, \& Weaver, 1973 , p. 53, pl. 5, fig. 8a, b; Gaston, 1974, p. 77 [hypotype examined].

Hypotype no. 00378, loc. PSUA0104.

Several specimens from the Neskowin Creek Section differ slightly from $B$. corrugata sensu stricto in that the early chambers are visible rather than indistinct. In all other respects however, these specimens are identical to $B$. corrugata and are included in this species. 
Bulimina curtissima Cushman \& Siegfus

Bulimina curtissima Cushman \& Siegfus, 1935, p. 93, pl. 14, fig. 9a, b; Cushman \& Parker, 1946, p. 94, pl. 22,

fig. 6a, b; Mallory, 1959, p. 189, pl. 15, fig. 18; Weaver \& Weaver, 1962, p. 31, pl. 9, fig. 4; Gaston, 1974, p. 77, [hypotype examined].

[Non] Bulimina curtissima Cushman \& Siegfus, Thoms, 1975 , pl. 2, fig. 17a, b.

Hypotype no. 00379 , 10c. PSUA0111.

Bulimina cf. B. curtissima Cushman \& Siegfus

Hypotype no. 00380 , loc. PSUA0107.

A single specimen from the Neskowin Creek Section compares

favorably with $B$. curtissima but has several spinose early

chambers instead of comparatively few.

Bulimina decurtata Cushman \& Stainforth

Bulimina decurtata Cushman \& Stainforth, 1947, p. 80, pl. 17,

fig. 11; Cushman \& Stainforth, 1951, p. 153, pl. 26,

fig. 47; Hornaday, 1961, p. 188, pl. 5, fig. la-c.

Hypotype no. $00381,10 c$. PSUA0118.

Bulimina decutata is easily confused with $B$. guayabalensis Cole var. ampla Cushman and Parker (1946, pl. 22, fig. 13) and variants of Globobulimina pacifica Cushman var. oregonensis Cushman, Stewart, and Stewart in which the later chambers fail to encompass the earlier chambers (Hornaday, 1961, pl. 7, fig. 4-7). It differs from B. guayabalensis var. amp $z a$ in that the last three chambers make up a larger portion of the test and from $G$. pacifica var. oregonensis in its apertural characteristics and chamber patterns. 
Bulimina elongata d'orbigny

Bulimina achwageri Yokoyama (?), Cushman \& Dusenbury, 1934, p. 62, pl. 8, fig. 1la, b.

Bulimina elongata d'Orbigny, Cushman \& Parker, 1937, p. 49, pl. 7, fig. 1-3; Cushman \& Parker, 1946, p. 108, pl. 25, fig. 14-17; Puri, 1953, p. 114, pl. 10, fig. 5, 6; Mallory, 1959, p. 190, pl. 16, fig. 12; Thoms, 1975, pl. 2, fig. 15.

Bulimina schwageri Yokoyama, Asano, 1952, p. 41, p1. 3, fig. 10; Asano, 1958, test-fig. 9.

Bulimina capitata Yokoyama (?), Smith, 1956, p. 95, p1. 13, fig. $7 a, b$.

Bulimina cf. B. schwageri Yokoyama, Fairchild, Wesendunk, \& Weaver, 1969, p. 54, pl. 11, fig. $14 a$, b.

Hypotype no. 00382 , loc. PSUA0123.

Most authors consider this species to be synonymous with B. schwageri (Cushman and Parker, 1946, p. 88). It is also similar to $B$. capitata but differs in possessing a decreasing chamber size in its later stages of growth.

Bulimina guayabalensis Cole var. ampla [?] Cushman \& Parker

Hypotype no. 00383 , 10c. PSUA0125.

Two poorly preserved specimens resemble $B$. guayabalensis var. ampla (Cushman \& Parker, 1946, p. 96, pl. 22, fig. 13) in outline but the sutural patterns and apertural characteristics are indistinct.

Bulimina instabilis [?] Cushman \& Parker

Hypotype no. 00384, 10c. PSUA0112.

A single fragmented specimen with chambers arranged in a regular series and low costae is questionably referred to this species. 
Bulimina jarvisi Cushman \& Parker

Bulimina jarvisi Cushman \& Parker, Cushman \& Parker, 1946, p. 100, pl. 23, fig. 10, pl. 24, fig. 1; DeLise, 1967, p. 34 , pl. 3, fig. $8 a$, b.

Hypotype no. 00385, 10c. PSUA0134.

This species closely resembles $B$. corrugata (Cushman and Parker, 1946, pl. 22, fig. 2a, b) and B. microcostata

Cushman \& Parker (Cushman \& Parker, 1946, pl. 22, fig. 9a-c) but has a much narrower, tapering test and fine irregular costae.

\section{Bulimina lirata Cushman \& Parker}

Bulimina lirata Cushman \& Parker, Cushman \& Simonson, 1944, p. 198, pl. 32, fig. 13; Cushman \& Parker, 1946, p. 95, pl. 22, fig. 10a-c; Smith, 1957, p. 174, pl. 24, fig. 13a-c; Mallory, 1959, p. 193, p1. 37, fig. la-c; Weaver \& Weaver, 1962, p. 32, pl. 9, fig. 8, 9; Rau, 1964, p. Gl8, pl. 5, fig. $17 a$, b; Fairchild, Wesendunk, \& Weaver, 1969, p. 54, pl. 11, fig. 9 .

[?] Bulimina lirata Cushman \& Parker, Gaston, 1974, p. 77, [hypotype examined].

Hypotype no. 00386 , lOc. PSUA0133.

Bulimina miarocostata Cushman \& Parker

Bulimina microcostata Cushman \& Parker, Cushman \& Parker, 1946, p. 95, pl. 22, fig. 9a-c; Mallory, 1959, p. 194, pl. 16, fig. 9; Hornaday, 1961, p. 188, pl. 6, fig. 2a-c; Sullivan, 1962, p. 274 , pl. 14, fig. 6a, b; Weaver, 1962, p. 379, pl. 4, fig. 2a-c, 4a-c; Kleinpell \& Weaver, 1963, p. 175, pl. 9, fig. 13; Weaver \& Molander, 1964, p. 189, pl. 8, fig. 18; Delise, 1967, p. 34, pl. 3, fig. 7; Fairchild, Wesendunk, \& Weaver, 1969, p. 54, pl. 11, fig. 10a, b; Smith, 1971 , p. 53, pl. 6, fig. 8a, b; Tipton, Kleinpell, \& Weaver, 1973, p. 53, pl. 5, fig. 9a, b; Gaston, 1974, p. 78, [hypotype examined].

Hypotype no. 00387, loc. PSUA0119. 


\section{Bulimina ovata d'orbigny}

[?] Bulimina ovata d'orbigny, Bagg, 1905, p. 22, p1. 3, fig. 1; Kleinpell, 1938, p. 255, pl. 12, fig. 14a, b.

Bulimina ovata d'Orbigny, Bagg, 1912, p. 38, pl. 9,

fig. 6a, b; Cushman, 1935 , p. 35 , pl. 13, fig. 15a, b $16 \mathrm{a}, \mathrm{b}$; Cushman \& Parker, 1937, p. 47, pl. 6, fig. 4a-c, 5a-c; Cushman \& Parker, 1946, p. 106, pl. 25, fig. 8a-c, 9a-c; Cushman, Stewart, \& Stewart, $1947 \mathrm{a}, \mathrm{p} .18, \mathrm{pl} .2$, fig. 8; Cushman, Stewart, \& Stewart, $1947 \mathrm{~b}$, p. $46, \mathrm{pl} .5$, fig. 9; Cushman \& Stainforth, 1951, p. 151, pl. 26, fig. 44; Puri, 1953, p. 115, pl. 16, fig. 11; Mallory, 1959, p. 195, pl. 16, fig. 4; Sullivan, 1962, p. 274, pl. 14, fig. 7a, b; Weaver, 1962, p. 380, pl. 4, fig. la-c; Kleinpell \& Weaver, 1963, p. 175, pl. 9, fig. 8; Weaver \& Molander, 1964, p. 189, pl. 8, fig. 19; Tipton, Kleinpell, \& Weaver, 1973, p. 53, pl. 5, fig. 10a, b; Gaston, 1974, p. 78, [hypotype examined]; Thoms, 1975, p1. 2, fig. 25.

[Non] Bulimina ovata d'Orbigny, Bergquist, 1942, p. 67, p1. 7, fig. 23.

Bulimina pupoides d'orbigny, Asano, 1958, pl. 10, fig. 15a, b, [not fig. 16].

Praeglobobulimina ovata (d'orbigny), Fairchild, Wesendunk, \& Weaver, 1969, p. 55, pl. 11, fig. 16; Murray \& Wright, 1974 , p1. 6, fig. 16,17 .

Hypotype no. 00388 , loc. PSUA0125.

This widely recorded species is closely related to $B$. pupoides d'orbigny (Cushman \& Parker, 1946, p1. 25, fig. 3-7) and in some cases it is almost impossible to separate the two. In the "Strata of Cascade Head", specimens of B. ovata are usually small, consist of few chambers, and possess oval shaped tests whereas $B$. pupoides is larger and has a slightly tapering test made up of numerous chambers. However, these are highly variable species and all of these characteristics may vary from specimen to specimen. 
Bulimina ovata [?] d'orbigny

Hypotype no. 00389, loc. PSUA0110.

Crushed individuals that resemble $B$. ovata are included here.

\section{Bulimina pachecoensis Smith}

Bulimina pachecoensis Smith, 1957, p. 175, pl. 24, fig. 14a-c; Thoms, 1975, pl. 2, fig. 20a, b.

Hypotype no. 00390, loc. PSUA0133.

These individuals have small tapering tests, inflated chambers in the last whorl, and costae extending onto the base of the final chambers.

$$
\text { Bulimina pachecoensis [?] Smith }
$$

Hypotype no. 00391 , loc. PSUA0132.

Several poorly preserved specimens are questionably referred to this species.

\section{Bulimina pupoides d'orbigny}

[?] Bulimina pupoides d'orbigny, Bagg, 1905, p. 22, pl. 3, fig. 2 .

Bulimina pupoides d'orbigny, Cushman \& Parker, 1937, p. 47, pl. 6, fig. 2a-c, 3a-c; Cushman \& Parker, 1946, p. 105, pl. 25, fig. 3-7; Bandy, 1944, p. 377, pl. 62, fig. 9a, b; Asano, 1952, p. 41, pl. 5, fig. 6a, b, 8, 9; Todd, 1957a, pl. 66, fig. 5, [not fig. 6]; Asano, 1958, pl. 10, fig. 16, [not fig. 15a, b] ; Mallory, 1959, p. 195, pl. 28, fig. 16a-c, [not pl. 36, fig. 17a-c].

[Non] (?) Bulimina pupoides d'Orbigny, Smith, 1957, p. 175, pl. 25, fig. la-c.

[Non] Bulimina pupoides Nuttall, Thoms, 1975, pl. 2, fig. $18 \mathrm{a}, \mathrm{b}$.

Hypotype no. 00392 , loc. PSUA0125.

See discussion under $B$. ovata. 
Bulimina cf. B. reussi Morrow [of Weaver]

Bulimina cf. B. reussi Morrow, Weaver, 1962, p. 380, pl. 3, fig. 6a-c; Weaver \& Molander, 1964, p. 190, pl. 9,

fig. $2 a, b$.

Hypotype no. $00393,10 c$. PSUAOI 34.

Specimens from the "Strata of Cascade Head" possess a greater number of chambers than $B$. reuss $i$ and appear identical to the forms figured by Weaver and Weaver and Molander.

\section{Bulimina schencki Beck}

Bulimina schencki Beck, 1943, p. 605, pl. 107, fig. 28, 33;

Cushman \& Parker, 1946, p. 98, pl. 30, fig. 16; Cushman,

Stewart, \& Stewart, $1947 \mathrm{c}$, p. 61, pl. 8, fig. 4, 5;

Cushman, Stewart, \& Stewart, $1947 \mathrm{e}$, p. 101, pl. 12,

fig. 10-12; Mallory, 1959, p. 196, pl. 16, fig. 15; Rau, 1964 , p. G18, pl. 5, fig. 10; Weaver \& Molander, 1964, p. 190, pl. 8, fig. 15; Gaston, 1974, p. 78, [hypotype examined].

Hypotype no. 00394 , 10c. PSUA0121.

This form resembles $B$. elongata d'orbigny but has more inflated chambers, a shorter tapering test, and a larger, rounded aperture (see discussion under $B$. elongata). Bulimina schencki also appears to be closely related to $B$. capitata Yokoyama. Examination of the primary types of these forms may show the two to be synonymous. 
Bulimina soulptilis Cushman

Bulimina sculptilis Cushman, 1923, p. 23, pl. 3, fig. 3; Cushman \& Schenck, 1928, p. 311, pl. 43, fig. 16; Franklin, 1944 , p. 314, pl. 45, fig. 15; Cushman \& Parker, 1946, p. 103 , pl. 24 , fig. $12 a-c$; Wilson, 1954, p. 139, pl. 15, fig. $12 a$, b; Smith, 1956 , p. 95, pl. 12, fig. 9, pl. 13, fig. 4a, b; Hornaday, 1961, p. 189, pl. 5, fig. 4a, b; Sullivan, 1962, p. 274 , pl. 14, fig. 8a, b; Kleinpell \& Weaver, 1963, p. 175, pl. 9, fig. 9, 10, 14; Weaver \& Molander, $1964, \mathrm{p}$. 190 , pl. 9 , fig. 3a, b, 4a, b; Delise, 1967, p. 35, pl. 3, fig. 9; Tipton, Kleinpell, \& Weaver, 1973, p. 53, p1. 5, fig. 1la, b; Gaston, 1974, p. 79, [hypotype examined]; Phillips, Tipton, \& Watkins, 1974, pl. 2, fig. $4 a, b, 5$.

[?] Bulimina sculptilis Cushman, Ujiie \& Watanabe, 1960, p. 130, pl. 2, fig. 15 .

Hypotype no. 00395,100 . PSUA0104.

$$
\text { Bulimina spp. }
$$

Specimens included here are insufficiently preserved to warrant specific designation. More than one species may be represented.

$$
\text { BuZimina [?] sp. }
$$

Several fragmented and crushed specimens seem best referred to this genus.

\section{Genus Globobulimina Cushman, 1927 \\ GLobobulimina pacifica Cushman}

Globobulimina pacifica Cushman, Kleinpell, 1938, p. 260, pl. 8, fig. 7; Beck, 1943 , p. 606, pl. 107, fig. 16; Franklin, 1944, p. 314, pl. 46, fig. 19; Cushman \& Parker, 1946 , p. 134 , pl. 29, fig. 37; Wilson, 1954, p. 139, pl. 15, fig. $14 a, b ;$ Asano, 1958, pl. 10, fig. 18; Mallory, 1959, p. 198, pl. 16, fig. 17; Weaver, 1962, p. 381, pl. 3, fig. $7 a, b$; Weaver \& Weaver, 1962, p. 32, pl. 9, fig. 15; Kleinpell \& Weaver, 1963, p. 176, pl. 9, fig. 11; Weaver \& Molander, 1964, p. 190, pl. 9, fig. 5; Fairchild, Wesendunk, \& Weaver, 1969, p. 55, pl. ll, fig. 16a, b; Smith, 1971, p. 54, pl. 7, fig. 1; Gaston, 1974, p. 79, [hypotype examined]. 
Globobulimina cf. G. pacifica Cushman, Rau, 1948, p. 171, pl. 30, fig. 5; Weaver \& Weaver, 1962, p. 32, pl. 9,

fig. 16; Smith, 1971, p. 54, pl. 7, fig. 2 .

Hypotype no. 00396, loc. PSUA0107.

Included within this highly variable species are individuals in which the final chambers fail to overlap and enclose the earlier chambers. These variants are difficult to differentiate from Bulimina decurtata and Bulimina guayabalensis var. amp $2 a$, as previously mentioned (see discussion under $B$. decurtata). Also, G. pacifica is easily crushed and care must be taken not to mistake distorted specimens for $G$. pacifica var. oregonensis Cushman, Stewart, and Stewart (Hornaday, 1961, p. 190, pl. 7, fig. 4a-c, 5a, b, 6a, b, $7 a-c)$ which is much broader and has contracted apertural and basal ends.

Globobulimina pacifica Cushman var. compressa Hornaday

GLobobulimina pacifica Cushman var. compressa Hornaday, 1961,

p. 189, pl. 7, fig. la, b, 2a, b, 3a, b.

Hypotype no. 00397, loc. PSUA0111.

Since G. pacifica Cushman is highly variable, individuals referred to $G$. pacifica var. compressa may fall within the range of variation of $G$. pacifica as suggested by Hornaday (1961, p. 189). Examination of an ontogenetic series may show the two to be synonymous. GLobobulimina [?] sp.

Hypotype no. 00398, loc. PSUA0120.

Flattened specimens are questionably referred to this genus. 
Genus Virgulina d'Orbigny, 1826

Virgulina bramlettei Galloway \& Morrey

Virgulina bramlettei Galloway \& Morrey, Cushman \& Laiming, 1931, p. 109, pl. 12, fig. 4a, b; Cushman, 1932a, p. 20, pl. 3, fig. 9a, b; Sullivan, 1962, p. 275, pl. 15,

fig. 3, 4a, b; Kleinpell \& Weaver, 1963, p. 176, pl.9,

fig. 5a-c; Gaston, 1974, p. 79, [hypotype examined].

Fursenkoina bramletti (Galloway \& Morrey), Fairchild,

Wesendunk, \& Weaver, 1969, p. 68, pl. 21, fig. 4, pl. 22, fig. 11 .

Fursenkoina bramlettei (Galloway \& Morrey), Smith, 1971,

p. 63, pl. 7, fig. 3a, b.

Hypotype no. 00399, 10c. PSUA0115.

This species differs from V. zetina Cole (Cushman, 1932a, p. 22, pl. 3, fig. 15) in that the last few chambers make up the greater portion of the test.

Virgulina zetina Cole

Virgulina zetina Cole, Cushman, 1932a, p. 22, pl. 3, fig. 15;

Cushman \& McMasters, 1936, p. 513, pl. 75, fig. 26; Howe, 1939 , p. 65, pl. 9, fig. 2; Weaver \& Weaver, 1962, p. 33, pl. 10, fig. l; Todd, 1966, pl. 5, fig. 5; Tipton, Kleinpell, \& Weaver, 1973, p. 54, pl. 5, fig. 13 .

Hypotype no. 00400, loc. PSUA0119.

See discussion under $V$. bramlettei.

Virgulina sp.

Hypotype no. 00401, loc. PSUA0133.

Several poorly preserved specimens resembling this genus are included here. 


\section{Genus Bolivina d'orbigny, 1839 \\ Bolivina basisenta Cushman \& Stone}

Bolivina basisenta Cushman \& Stone, Cushman, Stewart, \& Stewart, 1947c, p. 61, pl. 8, fig. 7a, b; Cushman, Stewart, \& Stewart, $1947 \mathrm{e}, \mathrm{p} .102, \mathrm{pl} .13$, fig. 6a, b; Cushman, Stewart, \& Stewart, 1949, p. 133, pl. 15, fig. 8a, b; Cushman \& Stainforth, 1951, p. 155, pl. 26, fig. 53, Weaver \& Weaver, 1962, p. 33, pl. 10, fig. 2a, b; Gaston, 1974 , p. 80, [hypotype examined].

Hypotype no. 00402 , loc. PSUA0121.

Specimens from the Neskowin Creek Section are characterized by a short test and spinose projections at the periphery of each chamber.

Bolivina basisenta Cushman \& Stone var. oregonensis Cushman, Stewart, \& Stewart

Bolivina basisenta Cushman \& Stone var. oxegonensis Cushman, Stewart, \& Stewart, 1949, p. 133, p1. 15, fig. 7a, b.

[?] Bolivina basisenta Cushman \& Stone var. oregonensis Cushman, Stewart, \& Stewart, Weaver \& Weaver, 1962, p. 33, pl. 10, fig. 3.

Hypotype no. 00403, loc. PSUA0121.

This variety differs from $B$. basisenta Cushman \& Stone in the narrower, more tapering test and lack of spinose projections at the margins of each chamber.

\section{Bolivina huneri Howe}

Bolivina huneri Howe, 1939, p. 66, pl. 9, fig. 3, 4; Beck, 1943 , p. 606, pl. 107, fig. 21; Mallory, 1959, p. 201, pl. 16, fig. 21; Weaver \& Molander, 1964, p. 191, pl.9, fig. 8 .

Hypotype no. 00404, loc. PSUA0101.

This species, restricted to one locality in the basal Neskowin Creek Section, is characterized by fine longitudinal costae and a small narrow test. 
Bolivina jacksonensis Cushman A Applin

Bolivina jacksonensis Cushman \& Applin, 1926, p. 167, pl. 7,

fig. 3, 4; Cushman, 1935 , p. 37, pl. 14, fig. 11-13;

Bergquist, 1942, p. 71, pl. 7, fig. 21, 27; Cushman \&

Simonson, 1944, p. 198, pl. 32, fig. 15; Cushman \&

Stainforth, 1951, p. 155, pl. 26, fig. 55, 56; Wilson,

1954, p. $140, \mathrm{pl}$. 15, fig. 15a, b; Hornaday, 1961, p. 190, pl. 6, fig. 8a, b; Weaver \& Molander, 1964, p. 191, pl. 9,

fig. 9; Gaston, 1974, p. 80, [hypotype examined].

[?] Bolivina jacksonensis Cushman \& Applin, Todd, 1957a, pl. 66, fig. 17 .

Hypotype no. 00405, loc. PSUA0125.

Bolivina kleinpelli Beck

Bolivina kleinpelli Beck, 1943, p. 606, p1. 107, fig. 39; Mallory, 1959, p. 201, pl. 16, fig. 20; Sullivan, 1962,

p. 276, pl. 15, fig. 6a, b; Weaver \& Molander, 1964,

p. 191, pl. 9, fig. 10; Gaston, 1974, p. 81, [hypotype examined].

Brizalina kleinpelli Beck, Fairchild, Wesendunk, \& Weaver,

1969, p. 52, pl. 9, fig. 7a, b.

Hypotype no. 00406 , loc. PSUA0137.

This distinctive species is easily recognized by its small size and sinuous sutures.

Bolivina scabrata [?] Cushman \& Bermudez

Hypotype no. $00407,10 c$. PSUA0116.

A single pyritized specimen from the Neskowin Creek Section is questionably referred to this species.

$$
\text { Bolivina sp. }
$$

Numerous crushed and fragmentary specimens are identifiable at only the generic level. More than one species is probably represented. 
Genus Loxostomum Ehrenberg, 1854

Loxostomum applinae (Plummer)

Loxostoma applinae (Plumer), Kline, 1943, p. 50, pl. 5, fig. 7 .

Loxostomum applinae (Plummer), Mallory, 1959, p. 202, pl, 17, fig. la, b; Weaver \& Weaver, 1962, p. 33, pl. 10, fig. 8; Thoms, 1975, pl. 2, fig. 24.

Hypotype no. 00408, loc. PSUA0137.

This species closely resembles Bifarina nuttalli Cushman and Siegfus (Mallory, 1959, pl. 29, fig. 2a, b) but differs in possessing fewer uniserial chambers (Mallory, 1959, p. 204). Individuals from the Salmon River Section are completely biserial and appear identical with the specimen figured by Mallory.

\section{Genus Bifarina Parker \& Jones, 1872 \\ Bifarina eleganta (Plummer)}

Bifarina eleganta (Plumer), Cole, 1938, pl. 2, fig. 3; Mallory, 1959, p. 204, p1. 17, fig. 2; Sullivan, 1962, p. 276, pl. 15, fig. Ila, b; Weaver, 1962, p. 381, p1. 4, fig. 8a, b; Weaver \& Weaver, 1962, p. 34, p1. 10,

fig. 9a, b; Gaston, 1974, p. 81, [hypotype examined].

Siphogenerinoides eleganta (Plummer), Kline, 1943, p. 46, pl. 4, fig. 18.

Hypotype no. 00409, 10c. PSUA0137.

Genus Chiloguembelina Loebich \& Tappan, 1956

Chiloguembelina cubensis (Palmer)

Gumbelina cubensis Palmer, Bergquist, 1942, p. 62, p1. 6, fig. 26, 27; Mallory, 1959, p. 205, pl. 17, fig. 3. 
Chiloguembelina cubensis (Palmer), Todd, 1966, p. I33, pl. 8, fig. la, b, 4; Todd, 1970, p. Al7, pl. 7, fig. 5.

Hypotype no. 00410, loc. PSUA0106.

See discussion under Karreriella chapapotensis (Cole) var. monumentensis Mallory.

Genus Uvigerina d'Orbigny, 1826

Uvigerina churchi Cushman \& Siegfus

Uvigerina churchi Cushman \& Siegfus, 1939, p. 29, pl. 6, fig. 16a, b; Mallory, 1959, p. 206, pl. 17, fig. 6; Hornaday, 1961, p. 191, pl. 8, fig. 1-4; Weaver, 1962, p. 381, pl. 5, fig. 2a, b; Weaver \& Weaver, 1962, p. 34, p1. 10, fig. 10; Rau, 1964, p. G20, pl. 6, fig. 9; Delise, 1967, p. 36, pl. 3, fig. 11; Gaston, 1974, p. 82, [hypotype examined] .

Hypotype no. 00411 , loc. PSUA0110.

Uvigerina churchi Cushman \& Siegfus

var. demicostata Mallory

Uvigerina churchi Cushman \& Siegfus var. demicostata Mallory, 1959, p. 207, pl. 17, fig. 10a, b; Weaver \& Molander, 1964, p. 191, pl. 9, fig. 11; Gaston, 1974, p. 82, [hypotype examined].

Hypotype no. 00412 , loc. PSUA0111.

\section{Uvigerina garzaensis Cushman \& Siegfus}

Uvigerina garzaensis Cushman \& Siegfus, 1939, p. 28, p1.6, fig. 15a, b; Cushman \& Simonson, 1944, p. 199, pl. 32, fig. 20, 21; Detling, 1946, p. 357, pl. 50, fig. 8a, b; Cushman, Stewart, \& Stewart, 1949, p. 133, pl. 15, fig. 9; Wilson, 1954, p. 141 , pl. 16, fig. 4a, b; Smith, 1956, p. 97, pl. 13, fig. 2a, b; Smith, 1957, p. 177, pl. 26, fig. 9a, b; Mallory, 1959, p. 208, pl. 37, fig. 2a, b; Hornaday, 1961, p. 191, pl. 8, fig. 5a, b; Suliivan, 1962, p. 277, pl. 16, fig. 2, 3a, b; Weaver, 1962 , p. 382, pl. 5, fig. 5a, b; Weaver \& Weaver, 1962, p. 34, pl. 10, fig. 11; Weaver \& Kleinpell, 1963, p. 177, pl. 10, fig. 7; Rau, 1964 , p. G20, pl. 6, fig. 6a, b; Weaver \& Molander, 1964, p. 191, pl. 10, fig. 1; Delise, 1967, p. 36, pl. 3, fig. 14; Fairchild, Wesendunk, \& Weaver, 1969, p. 56, pl. 12, fig. 9a, b; Smith, 1971 , p. 55, pl. 7, fig. 10; Tipton, Kleinpell, \& Weaver, 1973 , p. 56, pl. 6 , fig. 13a, b; Gaston, 1974, p. 83, [hypotype examined]. 
Hypotype no. 00413, 10c. PSUA0106.

Uvigerina garzaensis Cushman \& Siegfus

var. nudorobusta Mallory

Uvigerina garzaensis Cushman \& Siegfus var. nudo-robusta Mallory, 1959, p. 208, pl. 17, fig. 1la, b, 13a, b;

Sullivan, 1962, p. 277, pl. 16, fig. $4 a$, b; Weaver \&

Molander, 1964, p. 191, pl. 10, fig. 2; Delise, 1967, p. 36, pl. 3, fig. 15; Fairchild, Wesendunk, \& Weaver, 1969, p. 56, pl. 12 , fig. $4 a, b, 5 a, b, 8 a, b$.

Uvigerina garzaensis Cushman \& Siegfus subsp. nudorobusta Mallory, Smith, 1971, p. 55, pl.8, fig. 1 .

Hypotype no. $00414,10 c$. PSUA0108.

Uvigerina sp. A. [species nova]

(P1. 1, fig. 12, 13)

Test large, elongate, about twice as long as broad, rounded in section, fusiform, greatest width toward apertural end, initial end subacute to rounded, apertural end tapering to a slender neck; chambers distinct, numerous, inflated, increasing rapidly in size as added, overlapping in early portions, less so in later portions; sutures fairly distinct, depressed in later portions; wall ornamented with fine, longitudinal costae, continuous over adjacent chambers, frequently not extending onto the terminal chamber; aperture terminal, at end of short, stout neck.

Dimensions of hypotype: 00415 ; length $.58 \mathrm{~mm}$.; breadth $.17 \mathrm{~mm}$.

Hypotype no. 00415, loc. PSUA0134.

Individuals identical to these forms occur commonly in Narizian age deposits on the West Coast according to Rau (personal communication, 1976). This form differs recognizably from other previously recorded species in possessing an elongate test made up of inflated chambers ornamented by fine, longitudinal costae. It seems most closely related to $U$. churchi Cushman and Siegfus var. demicostata Mallory and $U$. kernensis Barbat and von Estorff (Smith, 1971, p. 56, pl. 8, 
fig. 5) and may represent an intermediate form between the two. The naming of this species must await publication in accordance with the International Code of zoological Nomenclature (1961, p. 9, art. 11a).

Uvigerina sp. A. [?] [species nova]

Hypotype no. 00416 , loc. PSUA0121.

Numerous individuals with fine longitudinal costae resemble this species but are badly crushed and cannot be positively identified.

$$
\text { Uvigerina sp. }
$$

Hypotype no. 00417, loc. PSUA0129.

A single unidentifiable internal mold from the Neskowin Creek section probably represents Uvigerina garaaensis var. nudorobusta Mallory (1959, pl. 17, fig. 1la, b).

Genus AnguZogerina Cushman, 1927

Angulogerina cf. A. occidentalis Cushman

Hypotype no. 00418 , 10c. PSUA0125.

A single specimen from the Neskowin Creek Section resembles Sullivan's figure of this species (1962, pl. 15, fig. 15a, b) but has more closely arranged chambers and is not as elongate as $A$. occidentalis.

Genus Trifarina Cushman, 1923

Trifarina advena Cushman var. californica Mallory

Trifarina advena Cushman var. californica Mallory, 1959,

p. 210 , pl. 17, fig. $14 \mathrm{a}-\mathrm{d}, \mathrm{pl}$. 29, fig. $6 \mathrm{a}-\mathrm{c}, \mathrm{pl} .40$,

fig. Ila, b; Weaver \& Weaver, 1962, p. 34, pl. 10, fig. 13;

Thoms, 1975, pl. 2, fig. 23a, b.

Hypotype no. 00419 , 10c. PSUA0105. 
Family ELLIPSOIDINIDAE

Genus Pleurostomelza Reuss, 1860

Pleurostomella acuta Hantken

Pleurostomella acuta Hantken, Smith, 1957, p. 179, pl. 26, fig. 12a-c; Mallory, 1959, p. 218, pl. 29, fig. 9a, b, pl. 37, fig. 7a-c.

Hypotype no. 00420, 10c. PSUA0111.

Pleurostomella cf. P. acuta Hantken

Hypotype no. 00421, loc. PSUA0109.

A single individual from the Neskowin Creek Section is shorter, has more robust chambers, and is less tapering than P. acuta.

Pleurostomelza gredalensis Cook

Pleurostomella gredalensis Cook, Mallory, 1959, p. 218, pl. 18, fig. 15, pl. 35, fig. 3a, b.

Hypotype no. 00422, loc. PSUA0109.

Pleurostomelza cf. P. gredalensis Cook

Hypotype no. 00423, 10c. PSUA0110.

A single specimen from the Neskowin Creek Section compares favorably with $P$. gredalensis except that the test is smaller and the final chamber is flattened.

\section{Pleurostomella paleocenica Cushman}

Pleurostomella cf. brevis var. alternans Schwager, Kline, 1943 , p. 50, pl. 6, fig. 23.

Pleurostomella paleocenica Cushman, 1947, p. 86, pl. 18, fig. 14, 15; Mallory, 1959, p. 219, p1. 18, fig. 17, Weaver \& Weaver, 1962, p. 35, pl. 1l, fig. 2a, b.

Pleurostomella (?) paleocenica Cushman, Fairchild, Wesendunk, \& Weaver, 1969, p. 67, pl. 21, fig. 12a, b.

Hypotype no. $00424,10 c$. PSUA0110. 
Pleurostomezza sp.

Hypotype no. 00425 , loc. PSUA0111.

Obscure apertural characteristics prevent a few fragmented specimens from being assigned at a specific level.

Genus ElZipsonodosaria A. Silvestri, 1900

Ellipsonodosaria cf. E. alexanderi Cushman [of Sullivan]

Ellipsonodosaria cf. E. alexanderi Cushman, Sullivan, 1962, p. 278, pl. 2l, fig. 1 .

Hypotype no. 00426 , loc. PSUA0126.

Specimens from the Neskowin Creek Section are identical to the individual figured by sullivan. This species is easily confused with Dentalina spinosa d'Orbigny, Nodosaria deliciae Martin, and Nodosaria cf. N. deliciae Martin of this study when specimens are weathered and the spines are worn down or missing. Elzipsonodosaria cf. E. alexanderi however, has very small, short spines and weathered specimens display smaller areas of spinal attachment as compared to the other species.

\section{Ellipsonodosaria plummerae Cushman}

Ellipsonodosaria plummerae Cushman, Cooper, 1944, p. 352, pl. 54, fig. 18, 19; Kline, 1943, p. 51, pl. 5, fig. 8; Mallory, 1959, p. 223, p1. 19, fig. 3; Weaver \& Weaver, 1962, p. 35, p1. 11, fig. 5; Thoms, 1975, p1. 2, fig. 26 .

Ellipsonodosaria sagrinensis (Bagg), Cooper, 1944, p. 352, pl. 54, fig. 22.

Nodogenerina aff. N. sagrinensis (Bagg), Smith, 1957, p. 172, pl. 24, fig. 2, 3 .

Hypotype no. 00427 , loc. PSUA0117.

Individuals referred to this species exhibit a large amount 
of variation in the number of spines ornamenting the chambers. All specimens however, show an increase in the number of spines as chambers are added.

Ellipsonodosaria sp. A. Cushman \& Siegfus

Ellipsonodosaria sp. A. Cushman \& Siegfus, Sullivan, 1962, p. 279, pl. 21, fig. 8, 9; Weaver \& Weaver, 1962, p. 35, p1. 11, fig. 8, 10, [? fig. 9]; Weaver \& Molander, 1964, p. 192, pl. 10, fig. 6 .

[?] Nodosaria clavaeformis Neugeboren, Fairchild, Wesendunk, \& Weaver, 1969, p. 35, pl. 4, fig. 16; Gaston, 1974, p. 67, [hypotype examined].

Nodosarella sp. A. (Cushman \& Siegfus), Fairchild, Wesendunk, \& Weaver, 1969, p. 68, pl. 21, fig. 11.

Hypotype no. 00428 , loc. PSUA0119.

Individuals with a rounded proloculus followed by an elongate second chamber seem best referred to this species. Genus Ellipsoidina Seguenza, 1859

$$
\text { ElZipsoidina [?] sp. }
$$

Hypotype no. 00429 , 10c. PSUA0109.

A single specimen with an ovate test and semilunate aperture is included under this designation. This individual however, may represent the genus Ellipsobulimina since both genera are identical externally and can only be separated by their internal characteristics.

$$
\begin{gathered}
\text { Family ROTALIIDAE } \\
\text { Genus Discorbis Lamarck, } 1804 \\
\text { Discorbis baintoni Mallory }
\end{gathered}
$$

Discorbis baintoni Mallory, 1959, p. 228, pl. 19, fig. 16a-c; Gaston, 1974 , p. 84, [hypotype examined]; Thoms, 1975, pl. 2, fig. $29 a-c$.

Hypotype no. $00430,10 c$. PSUA0127. 
Discorbis sp.

Hypotype no. 00431, loc. PSUA0107.

A single specimen from the Neskowin Creek Section is quite similar to D. devizensis Cook (Mallory, 1959, pl. 33,

fig. $12 \mathrm{a}-\mathrm{c})$ but possesses a poorly preserved umbilical area making positive identification impossible.

Genus Valvulineria Cushman, 1926

Valvulineria scrobiculata (Schwager)

Planulina scrobiculata (Schwager), Galloway \& Morrey, 1931, p. 346, pl. 39, fig. 8a-c.

Valvulineria cf. V. scrobiculata (Schwager), Beck, 1943 ,

p. 608, pl. 108, fig. 2l-23.

[?] Valvulineria scrobiculata (Schwager), Todd, 1957a, pl. 68, fig. 3a-c.

Valvulineria scrobiculata (Schwager), Mallory, 1959, p. 232, pl. 20, fig. 6a, b; Kleinpell \& Weaver, 1963, p. 178,

pl. 11, fig. la-c; Weaver \& Molander, 1964, p. 193, pl. 10, fig. 10a-c; Gaston, 1974, p. 84, [hypotype examined].

Hypotype no. 00432 , 10c. PSUA0105.

Valvulineria tumeyensis Cushman \& Simonson

Eponides cf. pygmea Hantken, Church, 1931, pl. A, fig. 1-3.

Valvulineria tumeyensis Cushman \& Simonson, $1944, \mathrm{p} .201$, p1. 33, fig. 13, 14; Hornaday, 1961, p. 191, p1. 9, fig. 1-4; Sullivan, 1962, p. 280 , pl. 17, fig. 3-6; Weaver, 1962 , p. 383, pl. 5, fig. 8a-ci Weaver \& Weaver, 1962, p. 36 , pl. 12, fig. la-c; Kleinpell \& Weaver, 1963, p. 178, pl. Il, fig. 2a-c; Weaver \& Molander, 1964, p. 193, pl. 11, fig. 1-3; Tipton, Kleinpel1, \& Weaver, 1973, p. 59, p1.9, fig. 3a-ci Gaston, 1974, p. 85 [hypotype examined] ; Phillips, Tipton, \& Watkins, 1974, pl. 2, fig. 7, 8.

[?] Valvulineria tumeyensis Cushman \& Simonson, Detling, 1946, p. 358, pl. 1l, fig. lla-c.

Valvulineria chirana Cushman \& Stone, Cushman, stewart, \& Stewart, 1949, p. 134, pl. 16, fig. 2a-c. 
Hypotype no. $00433,10 c$. PSUA0121.

This widely recorded species has been synonymized with $V$. chirana Cushman and Stone by many authors (Hornaday, 1961, p. 191; Kleinpell and Weaver, 1963, p. 178; Weaver and Molander, 1964, p. 178) in West Coast literature. Although the primary types were not observed, all published figures of $V$. chirana and $V$. tumeyensis examined during this investigation suggest the two forms are conspecific.

Valvulineria [?] sp.

Hypotype no. 00434 , loc. PSUA0121.

Numerous flattened, poorly preserved specimens seem best to represent this genus.

\section{Genus Gyroidina d'Orbigny, 1826 \\ Gyroidina condoni (Cushman \& Schenck)}

Eponides condoni Cushman \& Schenck, 1928, p. 313, pl. 44,

fig. 6, 7a-c; Beck, 1943, p. 608, pl. 108, fig. 27, 29, 31;

Detling, 1946, p. 358, pl. 50, fig. $12 \mathrm{a}-\mathrm{c}$.

Eponides condoni Cushman \& Schenck ?, Kleinpell, 1938, p. 317, pl. 6, fig. 8, 15, 17 .

Gyroidina condoni rotundiformis Cushman \& Simonson, 1944, p. 201, pl. 33, fig. 17-19; Delise, 1967, p. 38, pl. 4, fig. $2 a-c$.

Gyroidina cf. planulata Cushman \& Renz, Cushman, Stewart, \& Stewart, 1947 e, p. 102, pl. 12, fig. 15a-c; Fairchild, Wesendunk, Weaver, 1969, p. 73, pl. 23, fig. 7a-c.

Gyroidina condoni (Cushman \& Schenck), Wilson, 1954, p. 142, pl. 16, fig. 10a-c; Smith, 1956, p. 97, pl. 14, fig. 6a-c, 7a-c; Hornaday, 1961, p. 192, pl. 9, fig. 5a-c; Sullivan, 1962 , p. 280 , pl. 18, fig. 2a-c; Weaver, 1962, p. 383, pl. 6, fig. la-c; Kleinpel1 \& Weaver, 1963, p. 179, p1. 11, fig. 3a-c; Weaver \& Molander, 1964, p. 193, p1. 11, fig. 4a-c; Delise, 1967, p. 38, pl. 4, fig. 2a-c; Fairchild, Wesendunk, \& Weaver, 1969, p. 73, pl. 23, fig. 1la-c; Tipton, Kleinpell, \& Weaver, 1973, p. 60, pl. 9, fig. la-c; 
Gaston, 1974, p. 85, [hypotype examined]; Phillips, Tipton, \& Watkins, 1974, pl. 2, fig. 10a, b; Thoms, 1975, pl. 3, fig. $6 a-c$.

Hypotype no. 00435 , loc. PSUA0123.

Gyroidina orbicularis d'orbigny var. planata Cushman

Gyroidina orbicularis d'orbigny var. planata Cushman, 1935, p. 45, pl. 18, fig. 3a-c; Cushman \& Frizzell, 1943, p. 87, p1. 15, fig. 2-4; Cushman \& Stainforth, 1951, p. 158, pl. 27, fig. 22, 23; Mallory, 1959, p. 235, pl. 29, fig. 16a-c; Weaver, 1962, p. 383, pl. 6, fig. 4a-c; Weaver \& Weaver, 1962, p. 36, pl. 13, fig. la-c, 2a-c; Fairchild, Wesendunk, Weaver, 1969, p. 73, pl. 23, fig. 9a-c; Tipton, Kleinpell, \& Weaver, 1973, p. 60, pl. 9, fig. 2a-c.

Gyroidina orbicularis planata Cushman, Rau, 1948, p. 171, p1. 31, fig. 12-14; Rau, 1964, p. G21, p1. 6, fig. 10a-c; Gaston, 1974, p. 86, [hypotype examined]; Thoms, 1975, pl. 3, fig. 8a-c; Phillips, Welton, \& Welton, 1976, pl. 2, fig. 18a-c.

[Non] Gyroidina orbicularis d'orbigny var. planata Cushman, Sullivan, 1962, p. 280, pl. 18, fig. la-c.

[?] [Zapsus calami] Gyroidina orbicularis d'orbigny var. planta Cushman, Delise, 1967, p. 38, pl. 4, fig. 4a-c.

Hypotype no. 00436, IOc. PSUA0111.

Gyroidina soldanii d'orbigny

[Non] Rotalia soldanii (d'orbigny), Bagg, 1905, p. 51, pl. 10, fig. 5; Hanna, 1923, p. 325, pl. 59, fig. 3a-c.

[Non] Gyroidina soldanii d'orbigny, Church, 1931, pl. C, fig. $1-3$.

Gyroidina soldanii d'orbigny, Cushman \& Henbest, 1940, pl. 10, fig. 2a-c; Smith, 1956, p. 98, pl. 14, fig. 5a-c; Fairchild, Wesendunk, \& Weaver, 1969, p. 73 , pl. 24 , fig. la-c, 4a-c; Smith, 1971, p. 66, pl. 9, fig. la-c; Hornaday, 1972, p. 45, pl. 2, fig. la-c, $2 a-c$.

Gyroidina orbicularis d'orbigny var. planata Cushman, Sullivan, 1962, p. 280, pl. 18, fig. la-c.

Hypotype no. 00437, loc. PSUA0105.

The high variability of this species has led to much 
confusion in the West Coast literature (Smith, 1971, p. 66). Specimens assigned to this species from the Neskowin Creek and Salmon River Sections are plano-convex, average ten to eleven chambers in the final whorl and have limbate sutures that are nearly radial on the ventral side and slightly curved on the dorsal side.

This species is easily distingished from G. soldanii var. octocamerata Cushman and G. D. Hanna by its larger size, limbate sutures and greater number of chambers in the final whorl. It is much more difficult to differentiate from $G$. orbicularis var. planata Cushman since the two are closely related and often intergrade with one another. Gyroidina soldanii however, is usually subacute in peripheral view while G. orbicularis var. planata is more quadrate.

$$
\text { Gyroidina soldanii [?] d'orbigny }
$$

Hypotype no. $00438,10 c$. PSUA0129.

Crushed individuals that resemble this species are included here.

$$
\begin{gathered}
\text { Gyroidina soldanii d'orbigny var. octocamerata } \\
\text { Cushman \& G. D. Hanna }
\end{gathered}
$$

Gyroidina soldanii d'orbigny var. octocamerata Cushman \& G. D. Hanna, Cushman \& M. A. Hanna, 1927, p. 56, pl. 5, fig. 7; Cushman \& Schenck, 1928, p. 312, pl. 44, fig. 3-5; Cushman, 1935 , p. 45 , pl. 18, fig. 4a-c; Howe, 1939, p. 75, pl. 9, fig. 34-36; Cushman \& Stainforth, 1951, p. 158, pl. 27, fig. 20, 21; Mallory, 1959, p. 236, p1. 30, fig. la-c, pl. 42, fig. la-c; Weaver, 1962, p. $384, \mathrm{pl} .6$, fig. $6 a-c$.

Gyroidina soldanii octocamerata Cushman \& G. D. Hanna, Galloway \& Morrey, 1931, p. 343, pl. 39, fig. la-c; Smith, 1957, p. 181, pl. 27, fig. 5a-c; Gaston, 1974, p. 86, [hypotype examined]; Thoms, 1975, pl. 3, fig. 9a-c. 
Hypotype no. 00439 , loc. PSUA0117.

See discussion under G. soldanii.

Gyroidina cf. G. soldanii d'orbigny var. octocamerata Cushman \& G. D. Hanna

Hypotype no. 00440, loc. PSUA0125.

A few small individuals possess chambers which increase very rapidly in size such that the final whorl extends further ventrally than that of $G$. soldanii var. octocamerata sensu stricto.

$$
\text { Gyroidina spp. }
$$

Numerous individuals are insufficiently preserved to warrant specific designation. More than one species is probably represented.

Genus Eponides Montfort, 1808

$$
\text { Eponides Zotus (Schwager) }
$$

Globorotalia Lotus (Schwager), Bandy, 1944, p. 370, pl. 61,

fig. la-c.

Eponides Zotus (Schwager), Ellis, Messina, Charmatz, \& Ronai, 1969, fig. 1-31.

Hypotype no. 00441 , loc. PSUA0112.

A few specimens included under this designation are more biconvex than most individuals from the "Strata of Cascade Head" but fall within the range of variation of this species.

$$
\text { Eponides mexicana (Cushman) }
$$

[?] Rotalia soldanii (d'orbigny), Bagg, 1905, p. 51, pl. 10, fig. 5 .

Rotalia soldanii (d'orbigny), Hanna, 1923, p. 325, pl. 59, fig. 3a-c. 
Pulvinulina mexicana Cushman, 1925, p. 300, pl. 7, fig. 7, 8. Eponides mexicana (Cushman), Cushman \& M. A. Hanna, 1927, p. 54, pl. 5, fig. 8, 9; Howe, 1939, p. 75, pl. 9,

fig. 31-33, pl. 10, fig. 1-3; Smith, 1957, p. 182, pl. 27, fig. 10a-c; Mallory, 1959, p. 237, pl. 37, fig. lla-c, pl. 41, fig. 9a-c; Weaver \& Weaver, 1962, p. 37, pl. 13, fig. 4a-c; Gaston, 1974, p. 86, [hypotype examined]; Thoms, 1975, pl. 3, fig. 3a-c.

Eponides guayabalensis Cole var. yeguaensis Weinzierl \& Applin, Cushman \& Dusenbury, 1934, p. 62, pl. 9, fig. la-c. Eponides yeguaensis Weinzierl \& Applin, Cushman \& McMasters, 1936 , p. 514, pl. 76, fig. la-c; Beck, 1943, p. 608, pl. 108, fig. 1, 4.

Hypotype no. $00442,10 c$. PSUA0110.

Eponides mexicana [?] (Cushman)

Hypotype no. 00443, 1oc. PSUA0109.

Recrystallized and poorly preserved specimens are questionably referred to this species.

\section{Eponides umbonata (Reuss)}

Eponides umbonata (Reuss), Cushman, 1935, p. 48, pl. 19,

fig. 10a-c; Cushman \& Henbest, 1940, pl. 10, fig. la-c; Smith, 1957, p. 183, pl. 27, fig. 12a-c, 14a-c; Mallory, 1959, p. 239, p1. 30, fig. 3a-c, pl. 37, fig. lla-c;

Weaver \& Weaver, 1962, p. 37, pl. 14, fig. 2a-c, 3a-c; Smith, 1971, p. 61, p1. 9, fig. 3a-c; Gaston, 1974, p. 87, [hypotype examined]; Thoms, 1975, pl. 3, fig. 5a-c.

Eponides umbonatus (Reuss), Kleinpell, 1938, p. 322, pl. 6 , fig. 9, 12; Franklin, 1944, p. 316, pl. 47, fig. 5a-c; Cushman, Stewart, \& Stewart, $1947 \mathrm{a}$, p. 2l, pl. 3, fig. 2a-c, 3a-c; Cushman \& Stainforth, 1951, p. 159, pl. 27, fig. 28, 29; Todd, 1957a, pl. 68, fig. 9a, b; Smith, 1956, p.98, p1. 15, fig. 2a-c; Sullivan, 1962, p. 281, pl. 18,

fig. 7a-c, 8a-c; Kleinpell \& Weaver, 1963, p. 179, pl. 12, fig. 3a-c; Phillips, Welton, \& Welton, 1976, pl. 2,

fig. $15 a-c$.

Eponides cf. E. umbonata (Reuss), Cushman \& Simonson, 1944, p. 201, pl. 34, fig. 4, 5.

Hypotype no. 00444, loc. PSUA0116. 
Eponides umbonata [?] (Reuss)

Hypotype no. 00445 , 10c. PSUA0135.

Specimens with poorly preserved sutures and slightly lobate chambers are included here.

$$
\text { Eponides waltonensis [?] Applin \& Jordan }
$$

Hypotype no. 00446 , 10c. PSUA0100.

A single, recrystallized specimen from the basal Neskowin Creek Section resembles the specimen figured by Smith (1957, p1. 27, fig. 13a-c).

\section{Eponides spp.}

Individuals not referable to a specific level are included here. More than one species is probably represented.

Genus Parrelza Finlay, 1939

Parrezla tenuicarinata (Cushman \& Siegfus)

Pulvinulinella tenuicarinata Cushman siegfus, 1935, p. 95, pl. 14, fig. 1la-c; Weaver \& Weaver, 1962, p. 38, pl. 16, fig. la-c.

Parrezla tenuicarinata (Cushman \& Siegfus), Mallory, 1959, p. 240, pl. 21, fig. 5a-c.

Osangularia tenuicarinata (Cushman \& Siegfus), Fairchild, Wesendunk, \& Weaver, 1969, p. 74, p1. 24, fig. 5a-c.

[Non] Parrelza tenuicarinata (Cushman \& Siegfus), Gaston, 1974, p. 87, [hypotype examined].

Hypotype no. 00447, 10c. PSUA0109.

When complete, this species is very distinctive and easily recognized by its extremely limbate, slightly curved dorsal sutures, sigmoid ventral sutures, and very wide keel. Normally however, the later chambers become detached, resulting in an individual composed of only the early chambers. These 
smaller forms are easily confused with Epistomina eocenica Cushman and M. A. Hanna and many species of the genus Eponides. Nevertheless, upon close examination, these individuals can be separated by their distinctive dorsal and ventral sutures.

Genus Epistomina Terquem, 1883

Epistomina eocenica Cushman \& M. A. Hanna

Epistomina eocenica Cushman \& M. A. Hanna, 1927, p. 53, pl. 5, fig. 4, 5; Cushman \& Schenck, 1928, p. 313, pl. 44, fig. 9a-c; Cushman \& McMasters, 1936, p. 515, pl. 76, fig. 5a-c; Cushman \& Frizzell, 1943, p. 87, pl. 15, fig. 9, 10; Rau, 1948, p. 172, pl. 31, fig. 1-3; Cushman \& Stainforth, 1951, p. 159, pl. 27, fig. 32, 33; Gaston, 1974, p. 88, [hypotype examined].

[?] Epistomina eocenica Cushman \& M. A. Hanna, Cushman \& Simonson, 1944, p. 202, pl. 34, fig. 6; Sullivan, 1962, p. 282, pl. 20, fig. 2a-c; Fairchild, Wesendunk, \& Weaver, 1969, p. 75, pl. 25, fig. 2a-c.

Hypotype no. 00448 , loc. PSUA0121.

Although the specimens referred to this species vary greatly in size, they all compare well with the individual figured by Cushman and M. A. Hanna.

\section{Genus Cancris Montfort, 1808 \\ Cancris cocoaensis Cushman}

Cancris cocoaensis Cushman, Weaver, 1962, p. 384, pl. 6 , fig. $2 a-c$.

Ceratobulimina sp. Weaver \& Weaver, 1962, p. 38, pl. 15, fig. $4 a, b$.

Hypotype no. 00449 , loc. PSUA0137.

This species is characterized by a nearly biconvex test, five chambers in the final whorl, slightly curved dorsal sutures, and a broad apertural lip which projects over the 
umbilical region. Specimens from the "Strata of Cascade Head" have been compared with Weaver's hypotypes and are conspecific.

\section{Cancris joaquinensis Smith}

Cancris joaquinensis Smith, 1956, p. 98, pl. 15, fig. 5a-c, 6a-c; Rau, 1964, p. G22, p1. 6, fig. 13a-c; Weaver \& Molander, 1964, p. 194, pl. 12, fig. 5a-c; Delise, 1967, p. 39, p1. 4, fig. 5a-c; Tipton, Kleinpell, \& Weaver, 1973, p. 61, pl. 10, fig. 3a-c.

Valvulineria jacksonensis welcomensis Mallory, Gaston, 1974, p. 84, [hypotype examined].

Hypotype no. 00450, loc. PSUA0123.

Although the holotypes and paratypes of each were examined, this species is very difficult to separate from Valvulineria jacksonensis Cushman var. welcomensis Mallory (1959, pl. 20, fig. 3a-c, 5a-c). They are similar in outline, have approximately the same number of chambers in the final whorl, and both possess an apertural flap which extends into the umbilical region. However, $C$. joaquinensis differs in the nature of the spiral suture, displays more lobe shaped chambers, and has an elongate rather than rounded test.

Cancris [?] sp.

Hypotype no. 00451 , 10c. PSUA0105.

Specimens flattened during preservation are questionably referred to this genus. Several of these specimens may represent the genus Valvulineria. 
Genus Baggate 2 la Howe, 1939

Baggatella californica Mallory

Baggatella californica Mallory, 1959, p. 186, pl. 15,

fig. 15a, b; Weaver \& Weaver, 1962, p. 37, pl. 15,

fig. $2 a-c$.

Hypotype no. 00452, loc. PSUA0112.

Family AMPHISTEGINIDAE

Genus Asterigerina d'orbigny, 1839

Asterigerina crassaformis Cushman \& Siegfus

Asterigerina crassaformis Cushman \& Siegfus, 1935, p. 94,

p. 14, fig. I0a-c; Cushman \& Stainforth, 1951, p. 159,

pl. 27, fig. 34, 35; Smith, 1957, p. 186, pl. 28,

fig. 7a-c, 10a-c; Mallory, 1959, p. 242, pl. 37,

fig. 13a-c; Sullivan, 1962, p. 282, pl. 20, fig. 3a-c;

Weaver \& Weaver, 1962, p. 37, pl. 15, fig. 3a-c; Rau,

1964 , p. G22, p1. 6, fig. 14a-c; Fairchild, Wesendunk, \&

Weaver, 1969, p. $60, \mathrm{pl}$. 15, fig. 8a-c; Smith, 1971,

p. 58, pl. 9, fig. 4a-c; Tipton, Kleinpell, \& Weaver, 1973,

p. 61, pl. 10, fig. 2a-c.

Hypotype no. 00453, loc. PSUA0125.

Genus Amphistegina d'Orbigny, 1826

Amphistegina simiensis (Cushman \& McMasters)

Asterigerina simiensis Cushman \& McMasters, 1936, p. 515, pl. 77, fig. 1-4.

Amphistegina simiensis (Cushman \& McMasters), Bandy, 1944, p. 375, pl. 62, fig. 4a-c; Fairchild, Wesendunk, \& Weaver, 1969 , p. 63, pl. 18, fig. 10a, b.

Hypotype no. 00454 , loc. PSUA0100.

This species is similar to Amphistegina californica Cushman and M. A. Hanna (1927, pl. 6, fig. 3-5) but differs in its greater thickness and larger nodes which ornament the dorsal sutures. Recrystallized specimens from the basal Neskowin Creek Section are commonly weathered, exposing the secondary lobes. 
Family CASSIDULINIDAE

Genus Ceratobulimina Toula, 1915

Ceratobulimina alazanensis Cushman \& Harris

Ceratobulimina alazanensis Cushman \& Harris, 1927, p. 174, pl. 29, fig. 5a-c, pl. 30, fig. 3-5; Coryell \& Embich, 1937, p. 302, pl. 43, fig. 8a, b; Franklin, 1944, p. 317, pl. 47, fig. 8a, b; Cushman, $1946 \mathrm{~b}, \mathrm{p} .11 \mathrm{l}$, pl. 18,

fig. 17, 18; Weaver \& Molander, 1964, p. 194, pl. 13,

fig. la, b; Smith, 1971, p. 70, pl. 10, fig. 3a-c; Gaston, 1974 , p. 88, [hypotype examined].

[?] Ceratobulimina alazanensis Cushman \& Harris, Bergquist, 1942 , p. 91, pl. 9, fig. 17, 18.

Hypotype no. 00455, loc. PSUA0125.

This species somewhat resembles $C$. washburnei Cushman and Schenck (1928, pl. 45, fig. 1a-c) but is normally smaller in size and possesses different apertural characteristics; the aperture of $C$. washburnei is hidden by a thin arched plate whereas the aperture of $C$. alazanensis consists of an elongate slit in the center of the apertural face.

Genus A Zabamina Toulmin, 1941

A Zabamina scitula Bandy

A Zabamina scitula Bandy, Mallory, 1959, p. 226, pl. 19 ,

fig. $13 a-c$.

Hypotype no. 00456 , 10c. PSUA0116.

This species differs from Alabamina wilcoxensis Toulmin var. californica Mallory in its smaller size, few chambers, and radial sutures on the ventral side of the test which are recurved near the umbilical area. 
A Labamina wilcoxensis Toulmin var. californica Mallory

Alabamina wilcoxensis Toulmin var. californica Mallory, 1959, p. 227, pl. 19, fig. 11a-c, 12a-c; Gaston, 1974, p. 89, [hypotype examined].

[?] Alabamina wilcoxensis Toulmin var. californica Mallory, Thoms, 1975, pl. 3, fig. 12a-c.

Hypotype no. 00457, 10c. PSUA0106.

$$
\text { Alabamina sp. }
$$

Hypotype no. 00458 , 10c. PSUA0130.

A single specimen from the Cascade Head Road Section resembles $A$. wilcoxensis var. californica but obscured sutures prevent positive identification.

Genus Cassidulina d'Orbigny, 1826

\section{Cassidulina globosa Hantken}

Cassidulina globosa Hantken, Church, 1931, pl. C, fig. 4, 5; Cushman, 1935, p. 49, pl. 20, fig. 12a, b; Bergquist, 1942, p. 92, pl. 9, fig. 13, 14; Beck, 1943, p. 609, pl. 108, fig. 7, 13, 14; Cushman \& Simonson, 1944, p. 202, pl. 34, fig. 7; Detling, 1946, p. 358, pl. 5l, fig. 3a, b; Cushman, Stewart, \& Stewart, 1947d, p. 79, pl. 10, fig. 10a, b; Cushman, Stewart, \& Stewart, $1947 \mathrm{e}, \mathrm{p} .103, \mathrm{pl} .12$, fig. 14a, b; Cushman, stewart, \& Stewart, 1949, p. 135, pl. 16, fig. 3a, b; Asano, 1952, p. 42, pl. 4, fig. 3a-c; Wilson, 1954, p. 143, pl. 17, fig. la, b; Smith, 1956, p. 100, pl. 14, fig. 2a-c; Smith, 1957, p. 187, pl. 28, fig. 13a, b; Todd, 1957a, pl. 69, fig. 12a, b, pl. 73, fig. 15a, b; Asano, 1958, text-fig. 8a, b; Mallory, 1959, p. 226, pl. 33, fig. 1la, b; Hornaday, 1961, p. 193, pl. 10, fig. 3a-c; Sullivan, 1962, p. 283, pl. 20, fig. 5a, b; Weaver, 1962, p. 385, pl. 7, fig. la, b; Weaver \& Weaver, 1962, p. 38, pl. 16, fig. 3a-c; Kleinpell \& Weaver, 1963, p. 180, pl. 13, fig. 5a, b; Rau, 1964, p. G23, pl. 7, fig. 4a, b; Weaver \& Molander, 1964, p. 195, pl. 13, fig. 4a, b; Delise, 1967, p. 39, pl. 5, fig. 3a-c; Gaston, 1974, p. 89, [hypotype examined].

Globocassidulina globosa (Hantken), Fairchild, Wesendunk, \& Weaver, 1969, p. 69, pl. 22, fig. 15a, b; Smith, 1971, p. 63, pl. 9, fig. $6 a, b$.

Hypotype no. 00459, loc. PSUA0121. 


\section{Family CHILOSTOMELLIDAE \\ Genus AZZomorphina Reuss, 1850 \\ AZzomorphina macrostoma Karrer}

[?] AZZomorphina cf. macrostoma Karrer, Cushman \& McMasters, 1936, p. 516, pl. 76, fig. 7a, b.

[?] AZZomorphina macrostoma Karrer, Hornaday, 1961, p. 193, pl. 11, fig. 5a-c.

AZZomorphina macrostoma Karrer, Weaver \& Molander, 1964, p. 195, pl. 13, fig. 6a, b, 7a, b; Fairchild, Wesendunk, \& Weaver, 1969, p. 70, pl. 22, fig. 16a, b; Smith, 1971, p. 65, pl. 10, fig. 4a, b; Gaston, 1974, p. 90 [hypotype examined].

Hypotype no. $00460,10 c$. PSUA0113.

Genus Chizostomezzoides Cushman, 1926

Chizostomezloides eocenica Cushman

Chilostomelloides eocenica Cushman, 1926a, p. 78, pl. 11,

fig. 20a-c; Kline, 1943, p. 57, pl. 6, fig. 8 .

Hypotype no. $00461,10 c$. PSUA0111.

This distinctive species, common throughout the "strata of Cascade Head", is characterized by its aperture which is attached to the previous chamber and not as free as in Chilostomelloides oviformis (Sherborn and Chapman) (Sullivan, 1962, pl. 21, fig. 12a, b). To the author's knowledge, this species has never been recorded from Eocene deposits of the West coast.

ChilostomeZzoides oviformis [?] (Sherborn \& Chapman)

Hypotype no. 00462 , 10c. PSUA0121.

A single pyritized specimen from the Neskowin Creek section resembles sullivan's figure (1962, pl. 2l, fig. 12a, b) of $C$. oviformis but is badly fractured and missing test material. 
Chizostomezloides sp.

Hypotype no. 00463 , loc. PSUA0110.

Numerous flattened, poorly preserved individuals, which probably represent $c$. eocenica, are referred to this genus.

Genus PulZenia Parker \& Jones, 1862

Pullenia eocenica Cushman \& Siegfus

Pullenia eocenica Cushman \& Siegfus, 1939, p. 31, pl. 7, fig. la, b; Asano, 1958, pl. 1l, fig. 13a, b; Sullivan, 1962 , p. 284 , pl. 21, fig. 13a, b; Weaver \& Weaver, 1962, p. 39, pl. 17, fig. 2a, b; Fairchild, Wesendunk, \& Weaver, 1969, p. 72, pl. 23, fig. 8; Todd, 1970, p. Al4, pl. 5, fig. 5a, b; Smith, 1971, p. 64, pl. 10, fig. 5a, b; Tipton, Kleinpell, \& Weaver, 1973, p. 62, pl. 11, fig. $3 a, b$.

[?] Pullenia eocenica Cushman \& Siegfus, Mallory, 1959, p. 246 , pl. 30 , fig. $4 a, b$.

Pulzenia bulloides (d'Orbigny), Fairchild, Wesendunk, \& Weaver, 1969, p. 72, pl. 23, fig. la, b.

Hypotype no. 00464, loc. PSUA0116.

This species differs from Pullenia bulloides (d'orbigny)

in possessing an aperture that extends from umbilicus to umbilicus instead of being restricted to the central portion of the apertural face.

Pulzenia quinquezoba (Reuss)

[?] Pullenia quinqueloba (Reuss), Cushman, 1931, p. 57, pl. 10, fig. 4a, b; Cushman \& Henbest, 1940, pl. 10 , fig. 14; Delise, 1967, p. 40, pl. 5, fig. 4 .

Pulzenia quinqueloba (Reuss), Bergquist, 1942, p. 94, pl. 9, fig. 8; Kline, 1943, p. 57, pl. 6, fig. 4, 7; Todd, 1957a, pl. 78, fig. 2a, b; Mallory, 1959, p. 246, pl. 34, fig. la, b; Weaver \& Weaver, 1962, p. 39, p1. 17, fig. 3a, b; Weaver \& Molander, 1964, p. 196, pl. 14, fig. 2a, b; Tipton, Kleinpell, \& Weaver, 1973, p. 62, pl.11, fig. 2a, b; Murray \& Wright, 1974, p1. 18, fig. 13, 14; Thoms, 1975, pl. 4, fig. la, b. 
Hypotype no. $00465,10 c$. PSUA0137.

Pullenia salisburyi R. E. \& K. C. Stewart

Pullenia salisburyi R. E. \& K. C. Stewart, Beck, 1943,

p. 609, pl. 108, fig. 8, 12; Cushman \& Frizzell, 1943,

p. 88 , pl. 14 , fig. 16 ; Smith, 1957, p. 188, pl. 28,

fig. Ila, b; Todd, 1957b, pl. 29, fig. 10a, b; Mallory, 1959, p. 247, pl. 34, fig. 3a, b, pl. 38, fig. la, b;

Kleinpell \& Weaver, 1963, p. 180, pl. 13, fig. 6a, b; Todd, 1967 , p. A38, pl. 5, fig. 20a, b; Smith, 1971, p. 65, p1. 10, fig. 6a, b; Tipton, Kleinpell, \& Weaver, 1973, p. 62, pl. 11, fig. $4 a, b$.

Hypotype no. 00466, 10c. PSUA0105.

Pullenia sp.

Hypotype no. $00467,10 c$. PSUA0116.

A single specimen from the Neskowin Creek Section resembles Pullenia eocenica Cushman and Siegfus except for its much higher apertural face.

\title{
Pulzenia [?] sp.
}

Hypotype no. 00468, 10c. PSUA0115.

Poorly preserved individuals are questionably referred to this genus.

\author{
Family HANTKENINIDAE \\ Genus Pseudohastigerina Banner \& Blow, 1959 \\ Pseudohastigerina miera (Cole)
}

Nonion micrum Cole, Cushman, 1935, p. 30, pl. 11, fig. 14, 15;

Cushman, 1939 , p. 5, pl. 1, fig. 20-22; Howe, 1939, p. 58, pl. 7, fig. 22, 23; Todd, 1957a, pl. 65, fig. 26a, b;

Mallory, 1959, p. 181, pl. 15, fig. 3a-c, p1. 28,

fig. 12a, b; Thoms, 1975, pl. 4, fig. 3a, b.

Globigerinella micra (Cole), Sullivan, 1962, p. 285, pl. 22,

fig. $7 a, b, 8 a, b$. 
Hastigerina micra (Cole), Fairchild, Wesendunk, \& Weaver, 1969 , p. 60 , pl. 15, fig. la-c, 7a-c; Postuma, 1971, p. 228,7 fig. on p. 229 .

Globanomalina micra (Cole), Jenkins, 1971, p. 78, pl. 2, fig. 50-54.

Pseudohastigerina micra (Cole), Tipton, Kleinpell, \& Weaver, 1973, p. 63, pl. 12, fig. 2a, b; Gaston, 1974, p. 91, [hypotype examined].

Hypotype no. 00469 , loc. PSUA0111.

Family GLOBOROTALIIDAE

Genus Globorotalia Cushman, 1927

Globorotalia cf. G. cerroazulensis (Cole)

Hypotype no. 00470, loc. PSUA0137.

These individuals resemble Globorotalia cerroazulensis (Cole) (Postuma, 1971, 7 fig. on p. 185) but have smaller chambers and are more compressed in peripheral view. Globorotalia [?] spp.

Numerous poorly preserved specimens seem best included here. More than one species is probably represented. Family GLOBIGERINIDAE Genus GLobigerina d'orbigny, 1826 Globigerina ciperoensis angustiumbilicata Bolli Globigerina ciperoensis angustiumbilicata Bolli, Fairchild, Wesendunk, \& Weaver, 1969, p. 61, pl. 16, fig. 2a-c, 5a-c. Globigerina (Globigerina) ciperoensis angustiumbilicata Bolli, Jenkins, 1971, p. 144, pl. 15, fig. 451-453. Hypotype no. 00471, 1Oc. PSUA0109.

GLobigerina ciperoensis angustiumbilicata [?] Bolli Hypotype no. 00472 , loc. PSUA0136.

A single specimen from the Salmon River Section is similar 
in chamber arrangement to $G$. ciperoensis angustiumbilicata Bolli but the apertural and umbilical areas are too poorly preserved for positive identification.

$$
\text { GLobigerina cf. G. concinna Reuss }
$$

Hypotype no. $00473,10 c$. PSUA0109.

These specimens are identical in all respects with Tipton, Kleinpell, and Weaver's figure (1973, pl. 11, fig. 7a-c) of Globigerina concinna Reuss except for a smaller aperture. The true shape of the aperture however, is impossible to discern on most individuals since the apertural region is usually poorly preserved.

\section{GLobigerina decepta Martin}

Globigerina decepta Martin, Smith, 1957, p. 188, pl. 28,

fig. $14 a-c ;$ Mallory, 1959 , p. 249, pl. 34, fig. 5a-c, pl. 38, fig. 2a-c; Sullivan, 1962, p. 284 , pl. 22, fig. 3a-c; Weaver, 1962, p. 387, pl. 7, fig. 5a-c. Globorotalia decepta (Martin), Smith, 1971, p. 59, pl. 11, fig. la-c.

Hypotype no. 00474, loc. PSUA0109. Globigerina ouachitaensis Howe \& Wallace Globigerina ouachitaensis Howe \& Wallace, Howe, 1939, p. 83, pl. 12, fig. 18, 19; Sullivan, 1962, p. 285, pl. 22, fig. la-c, 2a-c; Postuma, 1971, p. 152, 7 fig. on p. 153.

[Non] GLobigerina ouachitaensis Howe \&allace, Hornaday, 1961, p. 194, pl. 12, fig. la-c.

Globigerina (GLobigerina) ouachitaensis Howe \& Wallace, Jenkins, 1971, p. 153, pl. 16, fig. 489, 490.

Hypotype no. 00475, loc. PSUA0108. 
Globigerina cf. G. ouachitaensis Howe \& Wallace

Hypotype no. 00476 , loc. PSUAOII5.

GLobigerina ouachitaensis Howe and Wallace, as described by Postuma (1971, p. 152), is ornamented with very fine spines on all chambers except for a smooth or nearly smooth final chamber. A number of individuals included in this designation are similar to this species except for a spinose final chamber.

\section{Globigerina praebulloides Blow}

Globigerina praebulloides Blow, Postuma, 1971, p. 268, 7 fig. on p. 269 ; Hornaday, 1972, p. 44 , pl. 2, fig. 6a-c; McKeel \& Lipps, 1975, p. 265, pl. 5, fig. 5a-c, 6a-c.

Hypotype no. 00477 , loc. PSUA0119.

$$
\text { Globigerina prasaepis Blow }
$$

GLobigerina prasaepis Blow, McKeel \& Lipps, 1975, p. 265, pl. 4, fig. la-c.

Hypotype no. 00478, loc. PSUA0121.

$$
\text { GLobigerina primitiva (Finlay) }
$$

Pseudogloboquadrina primitiva (Finlay), Jenkins, 1971, p. 170, pl. 18, fig. 555-561.

Globigerina primitiva (Finlay), Postuma, 1971, p. 154,

7 fig. on p. 155.

Hypotype no. 00479 , loc. PSUA0106.

Globigerina cf. G. yeguaensis Weinzierl \& Applin

Hypotype no. $00480,10 c$. PSUAO112.

Two specimens from the Neskowin Creek Section closely resemble McKeel and Lipps' figure (1975, pl. 2, fig. la-c) of G. cf. G. yeguaensis. These individuals differ from Subbotina 
[Non] GLobigerina Zinaperta Finlay, Fairchild, Wesendunk, \& Weaver, 1969, p. 62, pl. 16, fig. 3a-c, 4a-c.

GZobigerina (Subbotina) Iinaperta Finlay, Jenkins, 1971,

p. 162, pl. 18, fig. 551-554.

[?] GZobigerina cf. G. Zinaperta Finlay, Tipton, Kleinpell, \& Weaver, 1973, p. 63, pl. 12, fig. la-c.

Subbotina Zinaperta (Finlay), McKeel \& Lipps, 1975, p. 262, pl. 3, fig. $4 a-c$.

Hypotype no. 00483, loc. PSUA0105.

Subbotina linaperta [?] (Finlay)

Hypotype no. 00484 , 10c. PSUA0105.

Numerous individuals that lack the characteristic lip or possess poorly preserved apertural areas are included here.

Subbotina wilsoni (Cole)

Globorotalia bolivariana (Petters), Fairchild, Wesendunk, \& Weaver, 1969, p. 61, pl. 15, fig. 2a-c, 3a-c, 4a-c, 5a-c; Gaston, 1974, p. 93, [hypotype examined].

Subbotina wilsoni (Cole), McKeel \& Lipps, 1975, p. 265, pl. 5, fig. 2a-c.

Hypotype no. 00485 , 10c. PSUA0107.

This species is similar to Globorotalia bolivariana

(Petters) but is smaller and does not possess short spines on the surface of the test.

Subbotina wizsoni [?] (Cole)

Hypotype no. 00486 , 10c. PSUA0107.

Poorly preserved individuals that seem to resemble this species are questionably placed in this designation.

Genus GLobigerinatheka Bronnimann, 1952

Globigerinatheka index (Finlay)

Globigerinatheka (Globigerapsis) index index (Finlay), Jenkins, 1971, p. 187, p1. 22, fig. 641-645. 
Zinaperta (Finlay) (McKeel and Lipps, pl. 3, fig. 4-c) in their larger size and different position of the aperture.

$$
\text { Grobigerina spp. }
$$

Numerous poorly preserved specimens seem best referred to this genus. More than one species is probably represented. Genus Globorotaloides Bolli, 1957 Globorotaloides turgida (Finlay)

Globorotaloides turgida (Finlay), Jenkins, 1971, p. 191, p1. 23, fig. 656-658.

Hypotype no. 00481 , 10c. PSUA0109.

GLoborotaloides sp. [?] [of McKeel \& Lipps]

Globorotaloides sp. [?] McKeel \& Lipps, 1975, p. 262, pl. 3, fig. la-c, 2a-c.

Hypotype no. 00482 , 10c. PSUA0125.

A small individual ornamented with closely spaced spines and possessing five chambers in the last whorl appears to be very close if not conspecific with the specimens figured by McKeel and Lipps.

\section{Genus Subbotina Brotzen \& Pozaryska, 1961 Subbotina linaperta (Finlay)}

Globigerina triloculinoides Plummer, Beck, 1943, p. 609, pl. 108, fig. 2, 3; Detling, 1946, p. 359, pl. 51, fig. 2a, bi Gaston, 1974, p. 92, [hypotype examined]. Globigerina cf. G. triloculinoides Plummer, Smith, 1957, p. 189 , pl. 28, fig. $17 \mathrm{a}, \mathrm{b}$.

[?] Globigerina trizoculinoides Plummer, Mallory, 1959, p. 250 , pl. 30 , fig. $6 a, b$, [not pl. 38 , fig. $3 a, b]$; Sullivan, 1962, p. 285, pl. 22, fig. 5a, b, 6a, b, [not fig. 4a, b]; Weaver \& Weaver, 1962, p. 40, pl. 18, fig. 3a, b, [not fig. 4a, b] ; Weaver \& Molander, 1964, p. 197, pl. 15, fig. 2a, b, [not fig. 1]. 
Globigerapsis index (Finlay), Postuma, 1971, p. 136,

7 fig. on p. 137.

Globigerinatheka index (Finlay), McKeel \& Lipps, 1975,

p. 265, pl. 3, fig. 5a-c, pl. 5, fig. la-c.

Hypotype no. 00487 , loc. PSUA0115.

Family ANOMALINIDAE

Genus Anomalina d'orbigny, 1826

Anomalina crassisepta Cushman \& Siegfus

Anomalina crassisepta Cushman \& Siegfus, 1935, p. 95, pl. 14, fig. 12a-c; Mallory, 1959, p. 258, pl. 35, fig. 4a-c; Fairchild, Wesendunk, \& Weaver, 1969, p. 74, pl. 25, fig. 5a-c.

Hypotype no. 00488 , 1Oc. PSUA0105.

Anomalina dorri Cole var. aragonensis Nuttall

Anomalina dorri Cole var. aragonensis Nuttall, Cushman \&

Siegfus, 1939, p. 32, pl. 7, fig. 5; Mallory, 1959, p. 259, pl. 35, fig. 5a-c.

Anomalina dorri aragonensis Nuttall, Smith, 1957, p. 191, p1. 15, fig. la-c, 2a-c.

Hypotype no. 00489 , 10c. PSUA0119.

Anomalina garzaensis Cushman \& Siegfus

Anomalina garzaensis Cushman \& Siegfus, 1939, p. 32, pl. 7, fig. 3a-c; Mallory, 1959, p. 259, pl. 31, fig. la-c; Sullivan, 1962, p. 286, pl. 23, fig. 5a-c; Weaver \& Weaver, 1962, p. 41, pl. 20, fig. 3a-c; Weaver \& Molander, 1964, p. 198, pl. 16, fig. 4a-c; Tipton, Kleinpell, \& Weaver, 1973 , p. 64, pl. 12, fig. 3a-c; Gaston, 1974, p. 94, [hypotype examined].

[?] Anomalina garzaensis Cushman \& Siegfus, Delise, 1967, p. 4l, pl. 6, fig. 3a-c.

Anomalina cf. A. garzaensis Cushman \& Siegfus, Fairchild, Wesendunk, \& Weaver, 1969, p. 74, pl. 25, fig. 3a-c.

Hypotype no. $00490,10 c$. PSUA0111.

A single specimen from the Neskowin Creek Section appears 
similar to published figures of $A$. garzaensis except that it possesses ten chambers in the last whorl instead of the 12 to 15 described by Cushman and Siegfus. However, since this species exhibits a great variation in the number of chambers in the adult whorl, this small individual, which may represent a juvenile, is included under this designation. Anomalina cf. A. garzaensis Cushman \& Siegfus Hypotype no. 00491 , loc. PSUA0101.

A single individual from the basal Neskowin Creek Section has inflated chambers and a more lobate outline than published figures of A. garzaensis.

Anomalina regina Martin var. minor Smith

Anomalina regina Martin var. minor Smith, 1957, p. 192, pl. 29, fig. 2a-c, 4a-c; Mallory, 1959, p. 261, pl. 23, fig. $6 a-c$.

Hypotype no. 00492, loc. PSUA0121.

$$
\text { Anomalina sp. A. Cushman [of Mallory] }
$$

Anomalina sp. A. Cushman, Mallory, 1959, p. 263, pl. 23,

fig. 5a-c; Thoms, 1975, pl. 4, fig. 5a-c.

Hypotype no. 00493, loc. PSUA0111.

$$
\text { Anomalina [?] spp. }
$$

Flattened and poorly preserved specimens are included here. More than one species is probably represented. Genus PZanuZina d'orbigny, 1826

$$
\text { PZanuzina sp. }
$$

Hypotype no. 00494, 10c. PSUA0101.

Two compressed individuals from the basal Neskowin Creek Section seem best referred to this genus. 
Genus Cibicides Montfort, 1808

Cibicides cushmani Nuttall

Cibicides cushmani Nuttall, Cushman \& Siegfus, 1939, p. 33, pl. 7, fig. 7a-c; Mallory, 1959, p. 264, pl. 31, fig. 3a-c; Sullivan, 1962, p. 287, pl. 23, fig. 7a-ci Weaver \& Weaver, 1962, p. 41, pl. 2l, fig. 2a-ci Falrchild, Wesendunk, \& Weaver, 1969 , p. 65 , pl. 19, fig. 2a-c; Tipton, Kleinpell, \& Weaver, 1973, p. 65, pl. 13, fig. 2a-c; Thoms, 1975, p1. 4, fig. 9a-c.

[?] Cibicides cushmani Nuttall, Smith, 1957, p. 192, pl. 31, fig. 4a-c.

Cibicides hodgei Cushman \& Schenck, Hornaday, 1961, p. 194, pl. 13, fig. la-c.

Cibicidina cushmani (Nuttall), Smith, 1971, p. 62, pl. 12, fig. $2 a-c$.

Hypotype no. 00495, 10c. PSUA0137.

Cibicides cushmani, which ranges throughout most of the Eocene, is thought to be an older member of the Cibicides hodgei Cushman and Schenck Iineage (Mallory, 1959, p. 265). In Narizian age strata the two intergrade and are very difficult if not impossible in some instances to separate from each other. Cibicides cushmani seems to be more plano-convex and has a triangular shaped apertural face while $C$. hodgei is more rounded in peripheral view and possesses large distinctive pores in the test and sutures. There is also a resemblance between this species and Anomalina crassisepta Cushman and siegfus as noted by Beck (1943, p. 611). Anomalina crassisepta however, has nearly radial sutures on the ventral side of the test and is much thicker in peripheral view than C. cushmani. 
Cibicides felix Martin

Cibicides felix Martin, Mallory, 1959, p. 265, p1. 25, fig. 2a-c, [not pl. 31, fig. 4a-c]; Weaver \& Molander, 1964, p. 199, pl. 17, fig. 4a-c, [? fig. 3a-c].

[?] Cibicides felix Martin, Delise, 1967, p. 42, pl. 7, fig. la-c.

Hypotype no. 00496, 10c. PSUA0116.

Individuals from the "Strata of Cascade Head" are identical to the specimen figured by Mallory. This species is characterized by a plano-convex test consisting of seven to nine chambers in the last whorl, sigmoid sutures on the ventral surface, and a nearly circular test in outline (the last three chambers are usually lobate). Cibicides cf. C. felix Martin

Hypotype no. 00497, 10c. PSUA0116.

These individuals differ from $C$. $f e l i x$ in being slightly biconvex and usually possess a greater number of chambers in the final whorl.

\section{Cibicides Laimingi Mallory}

Cibicides laimingi Mallory, 1959, p. 266, pl. 24, fig. 3a-c, $4 \mathrm{a}-\mathrm{c}$.

[?] Cibicides laimingi Mallory, Weaver, 1962, p. 390, pl. 9, fig. 2a-c; Weaver \& Weaver, 1962, p. 4l, pl. 2l, fig. 4a-ci Fairchild, Wesendunk, \& Weaver, 1969, p. 66, pl. 20, fig. $4 a-c$.

Hypotype no. 00498 , 10c. PSUA0111.

\section{Cibicides Zaurisae Mallory}

Cibicides Zaurisae Mallory, 1959, p. 267, pl. 24, fig. 8a-c; Weaver \& Molander, 1964, p. 199, pl. 18, fig. la-c; Delise, 1967, p. 42, pl. 7, fig. 4a-c; Gaston, 1974, p. 95, [hypotype examined]. 
[?] Cibicides Laurisae Mallory, Thoms, 1975, pl. 4, fig. lla-c.

Hypotype no. 00499 , loc. PSUA0123.

\section{Cibicides Laurisae [?] Mallory}

Hypotype no. $00500,10 c$. PSUA0100.

Plano-convex specimens from the basal Neskowin Creek Section resemble $C$. Laurisae but are badly recrystallized and difficult to identify accurately.

\section{Cibicides lobatus d'orbigny}

Cibicides lobatus d'orbigny, Bandy, 1944, p. 374, p1. 62,

fig. la-c; Rau, 1964, p. G24, p1. 7, fig. 9a-c; Gaston,

1974, p. 95, [hypotype examined].

Hypotype no. 00501, loc. PSUA0105.

Cibicides macmastersi Beck

[3] Cibicides sp. A. Cushman \& McMasters, 1936, p. 516, pl. 76, fig. 9a-c.

Cibicides memastersi Beck, 1943, p. 612, p1. 109, fig. 2, 4, 15, Gaston, 1974, p. 95, [hypotype examined].

Hypotype no. 00502, loc. PSUA0101.

These individuals are distingished by their small size, plano-convex test, numerous chambers in the final whorl, and large ventral umbo.

Cibicides momastersi [?] Beck

Hypotype no. 00503, loc. PSUA0127.

Two small, fragmented specimens with a large ventral umbo and numerous chambers resemble this species but are too poorly preserved for positive identification. 
Cibicides ouachitaensis Howe \& Wallace var. alhambrensis Smith

Cibicides ouachitaensis Howe \& Wallace var. alhambrensis

Smith, 1957, p. 194, pl. 32, fig. 1a-c, 2a-c, 5a-c;

Mallory, 1959, p. 268, pl. 38, fig. 8a-c.

Hypotype no. $00504,10 c$. PSUA0136.

Although a few specimens are more compressed than the forms figured by Smith, most individuals included under this designation appear conspecific with both Smith and Mallory's figures.

\section{Cibicides pachyderma (Rzehak)}

Cibicides pachyderma (Rzehak), Galloway \& Morrey, 1931

p. 345, pl. 39, fig. 6a-c; Mallory, 1959, p. 268, pl. 31, fig. 5a-c; Gaston, 1974, p. 96, [hypotype examined]; Thoms, 1975 , pl. 4, fig. 13a-c.

Hypotype no. 00505, 10c. PSUA0137.

This species, although similar to Cibicides ouachitaensis Howe and Wallace var. alhambrensis Smith, is characterized by sutural thickenings over the umbilical area on the dorsal side of the test and nonlobate chambers.

Cibicides cf. C. praecursorius (Schwager)

Hypotype no. 00506, loc. PSUA0132.

A few specimens with irregularly sigmoid sutures somewhat resemble C. cf. C. praecursorius of Mallory (1959, pl. 25, fig. $5 a-c)$.

\section{Cibicides pseudoungerianus (Cushman)}

Cibicides pseudoungerianus (Cushman), Cushman, 1935, p. 52, pl. 23, fig. la-c; Coryell \& Embich, 1937, p. 302, pl. 43, fig. 15; Todd, 1953, p. 6, pl. 2, fig. 12a-c, 13a-c; Todd, $1957 a$, pl. 71, fig. 14a-c, pl. 80, fig. 10a-c; Fairchild, Wesendunk, \& Weaver, 1969, p. 66, p1. 20, fig. 3a-c.

Hypotype no. 00507, 10c. PSUA0106. 
Cibicides cf. C. pseudoungerianus (Cushman)

Hypotype no. 00508, loc. PSUA0109.

A few specimens from the Neskowin Creek Section resemble C. pseudoungerianus except for limbate sutures and a circular shaped test; the last three chambers of $C$. pseudoungerianus are lobate and normally possess depressed sutures.

Cibicides pseudowuelzorstorffi cole (?) [of Mallory]

Cibicides pseudowuellorstorffi Cole (?), Mallory, 1959,

p. 270 , pl. 26, fig. 4a-c.

Hypotype no. 00509, loc. PSUA0106.

Cibicides sandiegensis Cushman \& M. A. Hanna

Cibicides sandiegensis Cushman \& M. A. Hanna, 1927, p. 55, pl. 6, fig. 1, 2; Mallory, 1959, p. 270, pl. 25, fig. 7a-c.

[?] Cibicides sandiegensis Cushman \& M. A. Hanna, Thoms, 1975, pl. 4, fig. $14 a-c$.

Hypotype no. 00510, 10c. PSUA0119.

Cibicides cf. C. sandiegensis Cushman \& M. A. Hanna Hypotype no. 00511, 10c. PSUA0119.

These individuals differ from $C$. sandiegensis in that the umbilical area on the ventral side of the test is depressed rather than raised.

\section{Cibicides spiropunctatus Galloway \& Morrey}

Cibicides spiropunctatus Galloway \& Morrey, 1931, p. 346,

pl. 39, fig. 7a-c; Mallory, 1959, p. 270, pl. 25, fig. 3a-c;

Weaver, 1962, p. 390, p1.9, fig. 4a-c; Weaver \& Weaver, 1962, p. 41, pl. 22, fig. la-c; Fairchild, Wesendunk, \& Weaver, 1969, p. 67, pl. 21, fig. 14a-c; Tipton, Kleinpell, \& Weaver, 1973, p. 66, pl. 14, fig. 2a-c.

Hypotype no. 00512, 10c. PSUA0137.

Adult forms of this species can be easily confused with 
adult specimens of Cibicides martinezensis Cushman and Barksdale var. malloryi Smith (1957, pl. 21, fig. 9a-c). Cibicides spiropunctatus however, is nearly circular in outline except for the last two or three chambers which are lobate while $C$. martinezensis var. malloryi is completely lobate in outline.

\section{Cibicides spp.}

Abundant poorly preserved and fragmented specimens are included in this designation. More than one species is represented.

Genus Cibicidoides Brotzen, 1936

Cibicidoides coalingensis (Cushman \& G. D. Hanna)

Anomalina coalingensis Cushman \& G. D. Hanna, Cushman \&

Schenck, 1928, p. 315, pl. 45, fig. 6; Cushman \& Dusenbury, 1934, p. $63, \mathrm{pl} .9$, fig. 3a-c.

Anomalina cf. A. coalingensis Cushman \& G. D. Hanna, Cushman \& Simonson, 1944, p. 202, pl. 34, fig. 9, 10.

Cibicidoides coalingensis (Cushman \& G. D. Hanna), Wilson, 1954 , p. 145, pl. 17, fig. 4a-c; Smith, 1956, p. 101, pl. 16, fig. 2a-c; Smith, 1957, p. 195, pl. 30, fig. 2a-c, 3a-c; Mallory, 1959, p. 273, pl. 38, fig. lla-c; Fairchild, Wesendunk, \& Weaver, 1969, p. 75, pl. 25, fig. la-c; Gaston, 1974 , p. 96, [hypotype examined]; Thoms, 1975, pl. 4, fig. 7a-c.

Cibicides cocoaensis (Cushman), Gaston, 1974, p. 94, [hypotype examined].

Hypotype no. 00513, loc. PSUA0103.

$$
\text { Cibicidoides venezuelanus (Nuttall) }
$$

Cibicides venezuelanus Nuttall, Cushman \& Siegfus, 1939, p. 32, pl. 7, fig. 4a-c; Fairchild, Wesendunk, \& Weaver, 1969, p. 67, pl. 21, fig. 6a-c. 
Cibicidoides venezuelanus (Nuttall), Smith, 1957, p. 197, pl. 30, fig. la-c, 5a-c; Mallory, 1959, p. 274, pl. 31, fig. 6a-c; Gaston, 1974, p. 96, [hypotype examined].

[?] Cibicides venezuelanus Nuttall, Weaver, 1962, p. 391, p1. 9, fig. 3a-c.

Hypotype no. 00514, 10c. PSUA0137.

Cibicidoides [?] sp.

Hypotype no. 00515, loc. PSUA0135.

Two specimens from the Salmon River Section are questionably assigned to this genus.

Family PLANORBULINIDAE

Genus Gypsina Carter, 1877

Gypsina globula [?] (Reuss)

Hypotype no. 00516, 10c. PSUA0133.

A single specimen from the Salmon River Section appears conspecific with $G$. globula as figured by Cushman (1935, pl. 23, fig. 4, 5). More material however, is required for positive identification. To the author's knowledge, this species has not been recorded from Eocene deposits on the West Coast. 


\section{DESCRIPTIONS OF LOCALITIES}

All of the following microfossil localities are in the general vicinity of Cascade Head on the western flank of the central Oregon Coast Range, Lincoln and Tillamook Counties, Oregon. Map data are based on the United States Geological Survey Map of the Hebo Quadrangle (15 minute series, scale 1:62500, 1955). Locality numbers refer to the collections of the Earth Sciences Museum at Portland State University, Portland, Oregon. Localities are listed below in respect to their appropriate stratigraphic section.

Neskowin Creek Section (Fig. 1-A)

The major stratigraphic section was measured along Neskowin Creek which traverses the southwesternmost corner of Lincoln County, Oregon. Neskowin Creek flows in a northwesterly direction from a point north of U. S. Forest Service Road $S 532$ in the $\mathrm{SW}_{\frac{1}{4}}^{\frac{1}{4}} \mathrm{SW}_{\frac{1}{4}}, \mathrm{Sec}, 15, \mathrm{~T} 6 \mathrm{~S}, \mathrm{RlOW}$, to just south of the town of Neskowin, in Sec. 35, T6S, RllW, where it empties into the Pacific Ocean. The section was measured from an isolated outcrop referable to the Siletz River Volcanic Series 520 feet east of the bridge

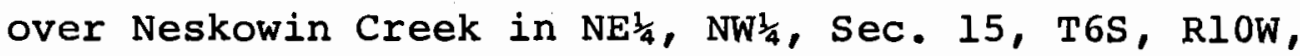
downstream to a point approximately 480 feet west of where

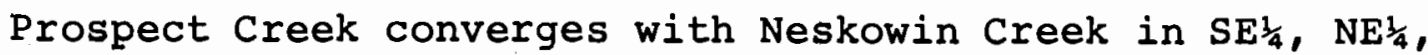


Sec. 6, T6S, Rlow. The stratigraphic datum for this section is placed seven feet stratigraphically below the base of a 13 foot wide outcrop of medium-gray, calcareous volcanic wacke (the base of the "Strata of Cascade Head") which is exposed directly under the bridge. The datum occurs 13 feet stratigraphically below the contact between this sandstone and a nine foot bed of olive-gray, tuffaceous siltstone. All foraminiferal samples were collected from the "Strata of Cascade Head" along the banks of Neskowin Creek and are depicted graphically in Figure 2.

$$
\text { Locality PSUA0100 }
$$

Located on the south bank of Neskowin Creek, 105 feet upstream from bridge, 10 feet stratigraphically above the base of the "Strata of Cascade Head" in medium-gray, calcareous volcanic wacke.

$$
\text { Locality PSUA0101 }
$$

Located on the south bank of Neskowin Creek, 7.5 feet stratigraphically above sample PSUA0100, in olive-gray, tuffaceous siltstone interbedded with calcareous volcanic wacke.

\section{Locality PSUA0102}

Located on the north bank of Neskowin Creek, 54.5 feet stratigraphically above sample PSUA010l, in medium-gray, calcareous volcanic wacke.

\section{Locality PSUA0103}

Located on the north bank of Neskowin Creek, 95.5 feet stratigraphically above sample PSUA0102, in medium-gray, calcareous volcanic wacke.

\section{Locality PSUA0104}

Located on the north bank of Neskowin Creek, at confluence with small south flowing tributary, 149 feet stratigraphically above sample PSUA0103, in light olive-gray, 
silty mudstone.

\section{Locality PSUA0105}

Located on the south bank of Neskowin Creek, 136 feet stratigraphically above sample PSUA0104, in olive-gray, silty mudstone.

\section{Locality PSUA0106}

Located on the south bank of Neskowin Creek, approximately 416 feet west of the Siuslaw National Forest Boundary, 254 feet stratigraphically above sample PSUA0105, in olive-gray mudstone.

\section{Locality PSUA0107}

Located on the north bank of Neskowin Creek, 54 feet stratigraphically above sample PSUA0106, in olive-gray, silty mudstone.

\section{Locality PSUA0108}

Located on the north bank of Neskowin Creek, seven feet stratigraphically above sample PSUA0107, in olive-gray, tuffaceous silty mudstone.

$$
\text { Locality PSUA0109 }
$$

Located on the south bank of Neskowin Creek, 379.5 feet stratigraphically above sample PSUA0108, in olive-gray, silty mudstone.

\section{Locality PSUA0110}

Located on the south bank of Neskowin Creek, 58.5 feet stratigraphically above sample PSUA0109, in olive-gray, silty mudstone.

\section{Locality PSUA0111}

Located on the north bank of Neskowin Creek, 45 feet stratigraphically above sample PSUA0110, in olive-gray mudstone.

\section{Locality PSUA0112}

Located on the south bank of Neskowin Creek, 74.5 feet stratigraphically above sample PSUA0lll, in olive-gray, tuffaceous silty mudstone. 
Locality PSUA0113

Located on the north bank of Neskowin Creek, approximately 705 feet west of junction of Neskowin Creek with Sloan Creek, 293 feet stratigraphically above sample PSUA0112, in olive-gray, micaceous shaly siltstone interbedded with olive-gray, tuffaceous volcanic wacke.

\section{Locality PSUA0114}

Located on the north bank of Neskowin Creek, 36 feet stratigraphically above sample PSUA0113, in dark-gray, micaceous mudstone interbedded with olive-gray, calcareous volcanic wacke.

Locality PSUA0115

Located on the south bank of Neskowin Creek, 28 feet stratigraphically above sample PSUA0114, in olive-gray, micaceous mudstone interbedded with medium dark-gray, calcareous volcanic graywacke.

\section{Locality PSUA0116}

Located on the south bank of Neskowin Creek, approximately 84 feet west of junction of Neskowin Creek and Lewis Creek, 219.5 feet stratigraphically above sample PSUA0115, in olive-gray mudstone.

$$
\text { Locality PSUA0117 }
$$

Located on the south bank of Neskowin Creek, 36.5 feet stratigraphically above sample PSUA01l6, in olive-gray, silty mudstone.

\section{Locality PSUA0118}

Located on the south bank of Neskowin Creek, 142.5 feet stratigraphically above sample PSUA0117, in olive-black, micaceous silty shale interbedded with olive-black, granular volcanic graywacke.

\section{Locality PSUAO119}

Located on the north bank of Neskowin Creek, 27 feet stratigraphically above sample PSUA0118, in dusky yellowishbrown, carbonaceous shaly siltstone interbedded with oliveblack volcanic graywacke. 
Locality PSUA0120

Located on the south bank of Neskowin Creek, 90 feet stratigraphically above sample PSUA0119, in olive-gray, micaceous siltstone interbedded with olive-gray, pebbly volcanic graywacke.

Locality PSUA0121

Located on the south bank of Neskowin Creek, 45 feet stratigraphically above sample PSUA0120, in olive-gray, micaceous siltstone interbedded with olive-black, pebbly volcanic graywacke.

\section{Locality PSUA0122}

Located on the north bank of Neskowin Creek, 73 feet stratigraphically above sample PSUA0121, in olive-gray, micaceous silty mudstone interbedded with olive-gray, volcanic graywacke.

\section{Locality PSUA0123}

Located on the south bank of Neskowin Creek, approximately 80 feet east of junction of Neskowin Creek and Jim Creek, 78.5 feet stratigraphically above sample PSUA0122, in olive-gray, carbonaceous shaly siltstone interbedded with olive-gray, micaceous carbonaceous shale.

\section{Locality PSUA0124}

Located on the north bank of Neskowin Creek, 212 feet stratigraphically above sample PSUA0123, in olive-gray, micaceous siltstone interbedded with olive-gray, micaceous wacke.

\section{Locality PSUA0125}

Located on the north bank of Neskowin Creek, 90 feet stratigraphically above sample PSUA0124, in olive-gray, micaceous siltstone.

Locality PSUA0126

Located on the north bank of Neskowin Creek, 7.5 feet stratigraphically above sample PSUA0125, in olive-gray, micaceous siltstone.

Locality PSUA0127

Located on the north bank of Neskowin Creek, 442 feet stratigraphically above sample PSUA0126, in olive-gray siltstone. 
Locality PSUA0128

Located on the south bank of Neskowin Creek, 285 feet stratigraphically above sample PSUA0127, in olive-gray, sandy siltstone.

\section{Locality PSUA0129}

Located on the south bank of Neskowin Creek, 710 feet east of junction of Neskowin Creek and Prospect Creek, five feet stratigraphically above sample PSUA0128, in olive-gray, sandy siltstone.

Cascade Head Road Section (Fig. 1-D)

This small supplementary section was measured along Cascade Head Road (U. S. Forest Service Road S6l) approximately half a mile west of its junction with Highway 101 in Tillamook County, Oregon. The section, situated in the $\mathrm{NE}_{\frac{1}{4}}, \mathrm{SE}_{\frac{1}{4}}, \mathrm{Sec} .7, \mathrm{~T} 6 \mathrm{~S}, \mathrm{R} 10 \mathrm{~W}$, was measured across the road and up the east side of the roadcut (this portion of

Cascade Head Road is constructed on the contact between the "Strata of Cascade Head" and the volcanics of the Nestucca Formation). The stratigraphic datum for this section is the base of an outcrop of olive-black, basaltic breccia (the base of the volcanics of the Nestucca Formation) exposed in the west bank of the roadcut. The sample was collected from the "Strata of Cascade Head".

Locality PSUA0130

Located in a roadcut on the east side of Cascade Head Road, about 10 feet above road surface, just under small ridge at the western end of the roadcut, approximately 10 feet stratigraphically below the volcanics of the Nestucca Formation, in light olive-gray, tuffaceous siltstone. 
micaceous arkosic wacke (at the base of the "Strata of Cascade Head"). All foraminiferal samples were collected from the "Strata of Cascade Head" along the banks of the Salmon River and are depicted graphically in Figure 3. Locality PSUA0132

Located on the south bank of the Salmon River, 12.5 feet stratigraphically above the base of the "Strata of Cascade Head", in medium-gray mudstone.

Locality PSUA0133

Located on the south bank of the Salmon River, 63.5 feet stratigraphically above sample PSUA0132, in olivegray mudstone.

\section{Locality PSUA0134}

Located on the north bank of the Salmon River, 12 feet stratigraphically above sample PSUA0133, in olivegray mudstone.

\section{Locality PSUA0135}

Located on the north bank of the Salmon River, 346 feet east of a logging road that crosses the river, 53 feet stratigraphically above sample PSUA0134, in olive-gray mudstone.

\section{Locality PSUA0136}

Located on the north bank of the Salmon River, 17 feet stratigraphically above sample PSUA0135, in olivegray mudstone.

\section{Locality PSUA0137}

Located on the north bank of the Salmon River, 34 feet stratigraphically above sample PSUA0136, in olivegray mudstone. 
Three Rocks Section (Fig. 1-C)

This small supplementary section was measured at the base of a sea cliff approximately half a mile northeast of the town of Three Rocks, Oregon. The section is situated in the SW $\frac{1}{4}, \mathrm{SW}_{\frac{1}{4}}, \mathrm{Sec} .14, \mathrm{~T} 6 \mathrm{~S}, \mathrm{RI} \mathrm{W}$, on the south side of Cascade Head at the mouth of the Salmon River. The stratigraphic datum for this section is the base of an outcrop of olive-black, basaltic breccia (at the base of the volcanics of the Nestucca Formation). The sample was collected from the "Strata of Cascade Head".

Locality PSUA0131

Located on the sea cliff on the south side of Cascade Head, about five feet above water level, approximately five feet stratigraphically below the volcanics of the Nestucca Formation in olive-gray mudstone.

Salmon River Section (Fig. 1-B)

This small stratigraphic section was measured along the Salmon River which traverses the southern portion of the Hebo Quadrangle in Lincoln County, Oregon. The section, located approximately two miles east of Rose Lodge, Oregon in the central portion of Sec. 29 and 30, T6S, R9W, was measured along the Salmon River from an outcrop referable to the Siletz River Volcanic Series approximately 3750 feet east of the logging road along the Treat River, upstream to a point about 1250 feet west of the western boundary of Van Duzer State Park. The stratigraphic datum for this section is at the base of an outcrop of medium-gray, 
PLATE 1

1. Vulvulina sp. A. n. sp. x54. Hypotype no. 00217. PSUA0134. Drawing from light microscope.

2. VuZvulina sp. A. n. sp. x33. Hypotype no. 00216. PSUA0134. Scanning electron micrograph.

3. Dentalina sp. A. n. sp. xl04. Hypotype no. 00316. PSUA0131. Single chamber. Drawing from light microscope.

4. Dentalina sp. A. n. sp. x50. Hypotype no. 00315. PSUA0131. Scanning electron micrograph.

5. Dentalina sp. B. xl10. Hypotype no. 00317. PSUA0134. Scanning electron micrograph.

6. Nodosaria (?) gyrata Mallory n. var. x88. Hypotype no. 00333 . PSUA0104. Scanning electron micrograph.

7. Nodosaria (?) gyrata Mallory n. var. x240. Hypotype no. 00333 . PSUA0104. Enlargement of figure 6 revealing the alternating fine costae. Scanning electron micrograph.

8. Nonion sp. A. n. sp. x167. Hypotype no. 00354. PSUA0119. Apertural view. Scanning electron micrograph.

9. Nonion sp. A. n. sp. xl25. Hypotype no. 00354 . PSUA0119. Side view. Scanning electron micrograph.

10. Elphidium sp. A. n. sp. xl66. Hypotype no. 00357. PSUA010l. Side view. Scanning electron microscope.

11. Elphidium sp. A. n. sp. x166. Hypotype no. 00357. PSUA0101. Oblique apertural view. Scanning electron micrograph.

12. Uvigerina sp. A. n. sp. x70. Hypotype no. 00415. PSUA0134. Side view. Scanning electron micrograph.

13. Uvigerina sp. A. n. sp. x252. Hypotype no. 00415. PSUAO134. Apertural view. Scanning electron micrograph. 


\section{BIBLIOGRAPHY}

ALLISON, R. C.

1959. Geology and Eocene megafaunal biostratigraphy of the Quimper Peninsula area, Washington: Unpub. M. S. thesis, Dept. Geol., Univ. Wash., 121 p., $13 \mathrm{pl} ., 15 \mathrm{fig}$.

ARNOLD, RALPH

1909. Environment of the Tertiary faunas of the Pacific Coast of the United States: Jour. Geol., v. 17, p. 509-533.

ASANO, $\mathrm{K}$.

1952. Paleogene Foraminifera from the Ishikari and Kushiro Coal-fields, Hokkaido: Short Papers Inst. Geol. Paleon., Tohoku Univ., n. 4, p. 23-46, pl. 3-5, 1 fig.

1958. Some Paleogene smaller Foraminifera from Japan: Sci. Rept., Tohoku Univ., 2nd Ser., v. 29, p. 43-75, pl. 8-13, 12 fig.

BAGG, R. M. , JR.

1905. Miocene Foraminifera from the Monterey Shale of California: U. S. Geol. Survey Bull. 268, 78 p., $11 \mathrm{pl}, 2 \mathrm{fig}$.

1912. Pliocene and Pleistocene Foraminifera from southern California: U. S. Geol. Survey Bull. $513,153 \mathrm{p} ., 28 \mathrm{pl}, 3 \mathrm{fig}$.

BAGLEY, R. E.

1959. Eocene stratigraphy of the Sekiu River area, Olympic Peninsula, Washington: Unpub. M. S. thesis, Dept. Geol., Univ. Wash., 130 p., 6 pl., 7 fig.

BALDWIN, E. M.

1955. Geology of the Mary's Peak and Alsea quadrangles, Oregon: U. S. Geol. Survey Oil and Gas Inv. Map OM 162.

1961. Geologic map of the lower Umpqua River area, Oregon: U.S. Geol. Survey Oil and Gas Inv. Map OM 204.

1964a. Geology of Oregon: 2nd ed., Ann Arbor, Michigan, Edwards Brothers, Inc., $165 \mathrm{p}$. 
BALDWIN, E. M.

1964b. Geology of the Dallas and Valsetz quadrangles, Oregon: revised ed., Ore. Dept. Geol. and Min. Ind. Bull. $35,56 \mathrm{p} ., 14 \mathrm{fig} ., 1$ map.

BALDWIN, E. M., BROWN, R. D., GAIR, J. E., and PEASE, M. H. , JR.

1955. Geology of the Sheridan and McMinnville quadrangles, oregon: U. S. Geol. Survey Oil and Gas Inv. Map OM 155.

BALDWIN, E. M., and ROBERTS, A. E.

1952. Geology of the spirit Mountain quadrangle, Oregon: U. S. Geol. Survey Oil and Gas Inv. Map OM 129.

BANDY, O. I.

1944. Eocene Foraminifera from Cape Blanco, Oregon: Jour. Paleon., v. 18, n. 4, p. 366-377, pl. 60-62.

1949. Eocene and Oligocene Foraminifera from Little Stave Creek, Clarke County, Alabama: Bull. Amer. Paleon., v. 32, n. 131, p. 5-210, $27 \mathrm{pl}$.

BANDY, O. L., and ARNAL, R. E.

1960. Concepts of foraminiferal paleoecology: Am. Assoc. Petroleum Geologists Bull., v. 44, n. 12, p. 1921-1932, 14 fig.

BEAULIEU, J. D.

1971. Geologic Formations of western Oregon: Ore. Dept. Geol. and Min. Ind. Bull. 70, $72 \mathrm{p}$.

BECK, R. S.

1943. Eocene Foraminifera from Cowlitz River, Lewis County, Washington: Jour. Paleon., v. 17, n. 6, p. 584-614, pl. 98-109, $4 \mathrm{fig}$.

BERGQUIST, H. R.

1942. Scott County Fossils; Jackson Foraminifera and Ostracoda: Miss. State Geol. Survey Bull. 49, $146 \mathrm{p} ., 11 \mathrm{pl}$.

BOGGS, S., JR., ORR, W. N., and BALDWIN, E. M.

1973. Petrographic and paleontologic characteristics of the Rickreall Limestone (Eocene) of northwestern Oregon: Jour. Sed. Petrology, v. 43, n. 3 , p. 644-654.

CARROLL, N. P.

1959. Upper Eocene and lower Oligocene biostratigraphy of the Hoko River area, northern Olympic Peninsula, Washington: Unpub. M. S. thesis, Dept. Geol., Univ. Wash., 101 p., 4 pl., 6 fig. 
CHURCH, C. C.

1931. Foraminifera of the Kreyenhagen Shale: Calif. Dept. Nat. Resources, Div. Mines, State Mineralogist's Rept., v. 27, n. 2, p. 202-213, pl. a-c.

1943. Descriptions of Foraminifera: Calif. State Div. Mines, Bull., n. 118, p. 182 .

COLE, W. S.

1938. Stratigraphy and micropaleontology of two deep wells in Florida: Fla. Geol. Survey Bull., n. 16, $73 \mathrm{p} ., 12 \mathrm{pl}, 3 \mathrm{fig}$.

COOPER, C. L.

1944. Smaller Foraminifera from the Porters Creek Formation (Paleocene) of Illinois: Jour. Paleon., v. 18 , n. 4, p. 343-354, pl. 54-55, 2 fig.

CORYELL, H. N, and EMBICH, J. R.

1937. The Tranquilla Shale (upper Eocene) of Panama and its foraminiferal fauna: Jour. Paleon., v. 11, n. 4 , p. 289-305, pl. 41-43, l fig.

CUSHMAN, J. A.

1913. A monograph of the Foraminifera of the North Pacific Ocean. Part 3, Lagenidae: U. S. Nat. Mus. Bull. 71, $125 \mathrm{p} ., 47 \mathrm{pl}$.

1918. Some Pliocene and Miocene Foraminifera of the coastal plain of the United States: U. S. Geol. Survey Bull. 676, $100 \mathrm{p}$., $31 \mathrm{pl}$.

1922. The Foraminifera of the Mint Spring Calcareous Marl Member of the Marianna Limestone: U. S. Geol. Survey Prof. Paper 129-F, p. 123-153, pl. 29-35.

1923. The Foraminifera of the Vicksburg Group: U. S. Geol. Survey Prof. Paper 133, p. 11-71, 8 pl.

1925. An Eocene fauna from the Moctezuma River, Mexico: Am. Assoc. Petroleum Geologists Bull., v. 9, n. 2, p. 298-303, pl. 6-8.

1926a. The genus Chilostomeila and related genera: Cushman Lab. Foram. Research, Contr., v. 1, pt. 4, p. 73-79, pl. 11 .

1926b. The Foraminifera of the Velasco Shale of the Tampico Embayment: Am. Assoc. Petroleum Geologists Bull., v. 10, n. 6, p. 581-612, pl. 15-21.

1927. Some new genera of the Foraminifera: Cushman Lab. Foram. Research, Contr., v. 2, pt. 4, p. 77-81.

1931. A preliminary report on the Foraminifera of Tennessee: Tenn. Div. Geol. Bull. 41,113 p., $13 \mathrm{pl}$.

1932a. Notes on the genus Virgulina: Cushman Lab. Foram. Research, Contr., v. 8, pt. 1, p. 7-23, p1. 2-3. 
CUSHMAN, J. A.

1932b. The genus Vulvulina and its species: Cushman Lab. Foram. Research, Contr., v. 8, pt. 4, p. 75-85, pl. 10.

1935. Upper Eocene Foraminifera of the southeastern United States: U. S. Geol. Survey Prof. Paper 181, $88 \mathrm{p} ., 23 \mathrm{pl}$.

1939. A monograph of the foraminiferal family Nonionidae: U. S. Geol. Survey Prof. Paper 191, 100 p., 20 pl.

1946a. Upper Cretaceous Foraminifera of the Gulf Region of the United States and adjacent areas: U. S. Geol. Survey Prof. Paper 206, 240 p., 66 pl.

1946b. The genus Ceratobulimina and its species: Cushman Lab. Foram. Research, Contr., v. 22, pt. 4, p. 107-117, pl. 17-19.

1947. Some new Foraminifera from the Paleocene of the southern United States: Cushman Lab. Foram. Research, Contr., v. 23, pt. 4, p. 81-86, pl. 17-18.

1950. Foraminifera, their classification and economic use: 4th ed., Cambridge, Mass., Harvard Univ. Press, $605 \mathrm{p} ., 55 \mathrm{pl} ., 31$ text pl., $9 \mathrm{fig}$.

CUSHMAN, J. A., and APPLIN, E. R.

1926. Texas Jackson Foraminifera: Am. Assoc. Petroleum Geologists Bull., v. 10, n. 2, p. 154-189, pl. 5-10.

CUSHMAN, J.A., and BARKSDALE, J. D.

1930. Eocene Foraminifera from Martinez, California: Stanford Univ., Dept. Geol., Contr., v. 1, n. 2, p. 55-73, pl. 11-12.

CUSHMAN, J. A., and DEADERICK, W. H.

1944. Cretaceous Foraminifera from the Marlbrook Marl of Arkansas: Jour. Paleon., v. 18, n. 4, p. 328-342, pl. 50-53.

CUSHMAN, J. A., and DUSENBURY, A. N., JR.

1934. Eocene Foraminifera of the Poway Conglomerate of California: Cushman Lab. Foram. Research, Contr., v. 10 , pt. 3, p. 51-65, pl. 7-9.

CUSHMAN, J. A., and ELLISOR, A. C.

1931. Some new Tertiary Foraminifera from Texas: Cushman Lab. Foram. Research, Contr., v. 7, pt. 3, p. 51-58, pl. 7 .

CUSHMAN, J. A., and FRIZZELI, D. L.

1943. Foraminifera from the type area of the Lincoln

Formation (Oligocene) of Washington State: Cushman

Lab. Foram. Research, Contr., v. 19, pt. 3,

p. 79-89, pl. 14-15, 1 fig. 
CUSHMAN, J. A., and HANNA, M. A.

1927. Foraminifera from the Eocene near San Diego, California: San Diego Soc. Nat. Hist., Trans., v. 5, n. 4, p. 47-65, pl. 4-6.

CUSHMAN, J. A., and HARRIS, R. W.

1927. Some notes on the genus Ceratobulimina: Cushman

Lab. Foram. Research, Contr., v. 3, pt. 4,

p. 171-176, pl. 29-30.

CUSHMAN, J. A., and HENBEST, L. G.

1940. Geology and biology of North Atlantic deep-sea cores. Part 2, Foraminifera: U. S. Geol. Survey Prof. Paper 196-A, p. 35-56, pl. 8-10, fig. 11-21.

CUSHMAN, J. A., and LAIMING, B.

1931. Miocene Foraminifera from Los Sauces Creek, Ventura County, California: Jour. Paleon., v. 5, n. 2, p. 79-120, pl. 9-14.

CUSHMAN, J. A., and MCMASTERS, J. H.

1936. Middle Eocene Foraminifera from the Llajas Formation, Ventura County, California: Jour. Paleon., v. 10, n. 6, p. 497-517, pl. 74-77, 4 fig.

CUSHMAN, J. A., and PARKER, F. I.

1937. Notes on some European Eocene species of Bulimina: Cushman Lab. Foram. Research, Contr., v. 13, pt. 2, p. 46-54, pl. 6-7.

1946. Bulimina and related foraminiferal genera: U. S. Geol. Survey Prof. Paper 210-D, p. 55-176, pl. $15-30$.

CUSHMAN, J. A., and SCHENCK, H. G.

1928. Two foraminiferal faunules from the Oregon Tertiary: Univ. Calif. Publ. Geol. Sci., v. 17, p. 305-325, pl. $42-45$.

CUSHMAN, J. A., and SIEGFUS, S. S.

1935. New species of Foraminifera from the Kreyenhagen Shale of Fresno County, California: Cushman Lab. Foram. Research, Contr., v. 11, pt. 4, p. 90-95, pl. 14.

1939. Some new and interesting Foraminifera from the Kreyenhagen Shales of California: Cushman Lab. Foram. Research, Contr., v. 15, pt. 2, p. 23-33, pl. 6-7.

CUSHMAN, J. A., and SIMONSON, R. R.

1944. Foraminifera from the Tumey Formation, Fresno County, California: Jour. Paleon., v. 18, n. 2, p. 186-203, pl. 30-34, 5 fig. 
CUSHMAN, J. A., and STAINFORTH, R. M.

1947. A new genus and some new species of Foraminifera from the upper Eocene of Ecuador: Cushman Lab. Foram. Research, Contr., v. 23, pt. 4, p. 77-80, pl. 17.

1951. Tertiary Foraminifera of coastal Ecuador: Part 1, Eocene: Jour. Paleon., v. 25, n. 2, p. 129-164, pl. 25-28, 4 fig.

CUSHMAN, J. A., STEWART, R. E. and STEWART, K. C.

1947a. Astoria Miocene Foraminifera from the northwest corner of Tenth Street and Harrison Avenue, Astoria, Clatsop County, Oregon: Ore. Dept. Geol. and Min. Ind. Bull., v. 36, pt. 1, p. 9-40, pl. $1-4$.

1947b. Astoria Miocene Foraminifera from Agate Beach, Lincoln County, Oregon: Ore. Dept. Geol. and Min. Ind. Bull., v. 36 , pt. 2, p. 4l-56, pl. 5-6, 1 fig.

1947c. Upper Coaledo (upper Eocene) Foraminifera from Yokam Point, Coos County, Oregon: Ore. Dept. Geol. and Min. Ind. Bull., v. 36, pt. 3, p. 57-70, pl. 7-8, 1 fig.

1947d. Lower Coaledo (upper Eocene) Foraminifera from Sunset Bay, Coos County, Oregon: Ore. Dept. Geol. and Min. Ind. Bull., v. 36, pt. 4, p. 71-92, pl. 9-1l, 1 fig.

1947e. Eocene Foraminifera from Helmick Hill, Polk County, Oregon: Ore. Dept. Geol, and Min. Ind. Bull., v. 36, pt. 5, p. 93-111, pl. 12-13, 1 fig.

1949. Upper Eocene Foraminifera from the Toledo Formation, Toledo, Lincoln County, Oregon: Ore. Dept. Geol. and Min. Ind. Bull., v. 36, pt. 6, p. 126-146, pl. 14-16, $1 \mathrm{fig}$.

DELISE, K. C.

1967. Biostratigraphy of the San Emigdio Formation, Kern County, California: Univ. Calif. Publ. Geol. Sci., v. $68,83 \mathrm{p.}, 7 \mathrm{pl} ., 8 \mathrm{fig}$.

DETLING, M. R.

1946. Foraminifera of the Coos Bay lower Tertiary, Coos County, Oregon: Jour. Paleon., v. 20, n. 4, p. $348-361, \mathrm{pl}$. 46-5l, 2 fig.

ELLIS, B. F., and MESSINA, A. R.

1940. Catalog of Foraminifera: Amer, Mus. Nat. Hist., Spec. Publ., v. 1-55.

ELLIS, B. F., MESSINA, A. R., CHARMATZ, R., and RONAI, L. E. 1969. Catalogue of index smaller Foraminifera. v. 3, Mesozoic-Tertiary benthonic Foraminifera: Am. Mus. Nat. Hist. 
FAIRCHILD, W. W., WESENDUNK, P. R., and WEAVER, D. W.

1969. Eocene and Oligocene Foraminifera from the Santa Cruz Mountains, California: Univ. Calif. Publ. Geol. Sci., v. 81, 144 p., 25 pl., 6 fig.

FENTON, C. L., and FENTON, M. A.

1928. Ecologic interpretation of some biostratigraphic terms: Amer. Midland Nat., v. 11, n. 1, p. 20-22.

FRANKLIN, E. S.

1944. Microfauna from the Carapita Formation of

Venezuela: Jour. Paleon., v. 18, n. 4, p. 301-319, pl. 44-48.

FRIZZELL, D. L.

1954. Handbook of Cretaceous Foraminifera of Texas: Univ. Texas, Bur. Econ. Geol., n. 22, 232 p., 21 pl., 2 fig.

GALLOWAY, J. J.

1933. A manual of Foraminifera: Bloomington, Indiana, Principia Press, $483 \mathrm{p} ., 42 \mathrm{pl}$.

GALLOWAY, J. J., and MORREY, M.

1931. Late Cretaceous Foraminifera from Tabasco, Mexico: Jour. Paleon., v. 5, n. 4, p. 329-354, pl. 37-40.

GASTON, L. R.

1974. Biostratigraphy of the type Yamhill Formation, Polk County, Oregon: Unpub. M. S. thesis, Dept. Earth Sci., Portland State Univ., 139 p., 4 fig.

GLAESSNER, M. F.

1963. Principles of micropaleontology: New York, Hafner Publishing Co., 296 p., 14 pl., 64 fig.

GODDARD, E. N., TRASK, P. D. , DE FORD, R. K., ROVE, O. N., SINGEWALD, J. T., JR. , and OVERBECK, R. M.

1970. Rock-color chart. Geol. Soc. America: Netherlands, Huyskes-Enschede.

HANNA, G. D.

1923. Some Eocene Foraminifera near Vacaville, California: Univ. Calif. Publ. Geol. Sci., v. 14, n. 9, p. 319-328, pl. 58-59.

HANNA, G. D., and HANNA, M. A.

1924. Foraminifera from the Eocene of Cowlitz River, Lewis County, Washington: Univ. Wash. Publ. Geol., v. 1, n. 4, p. 57-64, pl. 13 . 
HARRISON and EATON [firm]

1920. Report on investigations of oil and gas possibilities of western Oregon: Ore. Bur. Mines and Geology, Mineral Resources of Oregon, v. 3, n. 1 , p. 3-40.

HORNADAY, G. R.

1961. Foraminifera from the Sacate Formation south of Refugio Pass, Santa Barbara County, California: Univ. Calif. Publ. Geol. Sci., v. 37, n. 3, p. $165-232,13 \mathrm{pl} ., 6$ fig.

1972. Oligocene smaller Foraminifera associated with an occurrence of Miogypsina in California: Jour. Foram. Research, v. 1, n. 1, p. 35-46, 2 pl., 2 fig.

HOWE, H. V.

1939. Louisiana Cook Mountain Eocene Foraminifera: La. Geol. Survey Bull., n. 14, 122 p., 14 pl.

ISRAELSKY, M. C.

1951. Foraminifera of the Lodo Formation, central California: U. S. Geol. Survey Prof. Paper 240-A, 29 p., 11 pl., 2 fig.

1955. Foraminifera of the Lodo Formation, central California: U. S. Geol. Survey Prof. Paper 240-B, p. 31-79, pl. 12-19, fig. 3 .

JENKINS, D. G.

1971. New Zealand Cenozoic planktonic Foraminifera: New Zealand Geol. Survey Bull. 42, 278 p., 23 pl., 2 fig.

KLEINPELL, R. M.

1938. Miocene stratigraphy of California: Tulsa, Okla., Am. Assoc. Petroleum Geologists, 450 p., 22 pl., 14 fig.

KLEINPELL, R. M. , and WEAVER, D. W.

1963. Oligocene biostratigraphy of the Santa Barbara Embayment, California: Univ. Calif. Publ. Geol. Sci., v. 43, 250 p., 38 pl., 8 fig.

KLINE, V. H.

1943. Clay County Fossils; Midway Foraminifera and Ostracoda: Miss. State Geol. Survey Bull. 53, $98 \mathrm{p} ., 8 \mathrm{pl}$.

LOEBLICH, A. R., JR., and TAPPAN, HELEN

1964. Sarcodina, chiefly "Thecamoebians" and Foraminiferida, in Moore, R. C. (ed.), Treatise on invertebrate paleontology: Geol. Soc. America, Part C, Protista, v. 1-2, p. Cl-C900, 653 fig. 
MALLORY, V. S.

1959. Lower Tertiary biostratigraphy of the California Coast Ranges: Tulsa, Okla., Am. Assoc. Petroleum Geologists, 416 p., 42 pl., 7 fig.

MARKS, P., JR.

1951. A revision of the smaller Foraminifera from the Miocene of the Vienna Basin: Cushman Found.

Foram. Research, Contr., v. 2, pt. 2, p. 33-73, pl. 5-8.

MARTIN, L. T.

1943. Eocene Foraminifera from the type Lodo Formation, Fresno County, California: Stanford Univ. Publ. Geol. Sci., v. 3, n. 3, 35 p., pl. 3-9, 3 fig.

MAYR, ERNST

1969. Principles of systematic zoology: San Francisco, McGraw-Hill Book Company, 428 p.

MCKEEL, D. R., and LIPPS, J. H.

1975. Eocene and Oligocene planktonic Foraminifera from the central and southern Oregon Coast Range:

Jour. Foram. Research, v. 5, n. 4, p. 249-269,

5 pl., 3 fig.

MURRAY, J. W. , and WRIGHT, C. A.

1974. Palaeogene Foraminiferida and palaeoecology, Hampshire and Paris Basins and the English Channel: Palaeon. Assoc. London, Spec. Paper, n. 14, 129 p., 20 pl., 47 fig.

NATLAND, M. L.

1933. The temperature and depth-distribution of some Recent and fossil Foraminifera in the southern California region: Bull. Scripps. Inst. Oceanog., Tech. Ser., v. 3, n. 10, p. 225-230, I chart.

PHILLIPS, F. J., TIPTON, ANN, and WATKINS, RODNEY

1974. Outcrop studies of the Eo-Oligocene Tumey Formation, Monocline Ridge, Fresno County, California: Pacific Section, SEPM, Fieldtrip Guidebook, p. 99-131, 3 pl., 6 fig.

PHILLIPS, F. J., WELTON, B. J., and WELTON, J. E.

1976. Paleontologic studies of the middle Tertiary Skooner Gulch and Gallaway Formations at Point Arena, California: Pacific Section, AAPG-SEPM Guidebook, p. 137-152, 5 pl., 5 fig. 
PHLEGER, F. B.

1951. Ecology of Foraminifera, northwest Gulf of Mexico. Part 1, Foraminifera distribution: Geol. Soc. America, Mem. 46,88 p., 33 fig.

PHLEGER, F. B., and PARKER, F. L.

1951. Ecology of Foraminifera, northwest Gulf of Mexico. Part 2, Foraminifera species: Geol. Soc. America, Mem. 46, 64 p., $20 \mathrm{pl}$.

POSTUMA, J. A.

1971. Manual of planktonic Foraminifera: Amsterdam, Elsevier Publishing Company, $420 \mathrm{p}$.

PURI, H. S.

1953. Contribution to the study of the Miocene of the Florida Panhandle. Part 2, Foraminifera: Fla. Geol. Survey Bull., n. 36, p. $71-213,30$ pl.

1957. Stratigraphy and zonation of the Ocala Group. Part 2, Foraminifera: Fla. Geol. Survey Bull., n. 38, p. $93-184,15$ pl.

RAU, W. W.

1948. Foraminifera from the Porter Shale (Lincoln Formation), Grays Harbor County, Washington: Jour. Paleon., v. 22, n. 2, p. 152-174, pl. 27-31, 1 fig.

1964. Foraminifera from the northern Olympic Peninsula, Washington: U. S. Geol. Survey Prof. Paper 374-G, 33 p., 7 pl., 2 fig.

RAUP, D. M., and STANLEY, S. M.

1971. Principles of paleontology: San Francisco, W. H. Freeman and Company, $388 \mathrm{p}$.

SCHENCK, H. G., and KLEINPELL, R. M.

1936. Refugian Stage of Pacific Coast Tertiary: Am. Assoc. Petroleum Geologists Bull., v. 20, n. 2, p. 215-225.

SCHENK, E. T., and MCMASTERS, J. H.

1956. Procedure in taxonomy: 3rd ed., Stanford, Calif., Stanford Univ. Press, 119 p.

SCHLICKER, H. G., DEACON, R. J., OLCOTT, G. W., and BEAULIEU, J. D.

1973. Environmental Geology of Lincoln County, Oregon: Ore. Dept. Geol. and Min. Ind. Bull. 81, 171 p., 6 maps.

SMITH, B. Y.

1957. Lower Tertiary Foraminifera from Contra costa County, California: Univ. Calif. Publ. Geol. Sci., v. 32 , n. 3, p. 127-242, pl. 17-32, 5 fig. 
SMITH, H. P.

1956. Foraminifera from the Wagonwheel Formation, Devils Den district, California: Univ. Calif. Publ. Geol. Sci., v. 32, n. 2, p. 65-126, pl. 9-16, 3 fig.

SMITH, P. B.

1964. Ecology of benthonic species: U. S. Geol. Survey Prof. Paper 429-B, 55 p., 6 pl., 17 fig.

SMITH, R. K.

1971. Foraminiferal studies in the lower and middle Tertiary of Soquel Creek, Santa Cruz County, California: Univ. Calif. Publ. Geol. Sci., v. 91, 111 p., 12 pl., 6 fig.

SNAVELY, P. D., JR., and BALDWIN, E. M.

1948. Siletz River Volcanic Series, northwestern Oregon: Am. Assoc. Petroleum Geologists Bull., v. 32, n. 5, p. 805-812, 4 fig.

SNAVELY, P. D., JR., and MACLEOD, N. S.

1974. Yachats Basalt-an upper Eocene differentiated volcanic sequence in the Oregon coast Range: U. S. Geol. Survey, Jour. Research, v. 2, n. 4, p. $395-403,3$ fig.

SNAVELY, P. D., JR., MACLEOD, N. S., and RAU, W. W.

1969a. Geology of the Newport area, Oregon: The Ore Bin, v. 31, n. 2, p. 25-48, 6 fig.

1969b. Geology of the Newport area, Oregon: The ore Bin, v. 31, n. 3, p. 49-71, 2 pl., 9 fig.

SNAVELY, P. D. , JR. , MACLEOD, N. S., RAU, W. W. , ADDICOTT, W. O., and PEARL, J. E.

1975. Alsea Formation-an oligocene marine sedimentary sequence in the Oregon Coast Range: U. S. Geol. Survey Bull. 1395-F, 21 p., 7 fig.

SNAVELY, P. D., JR., MACLEOD, N. S., and WAGNER, H. C.

1972. Preliminary bedrock geologic map of the Cape Foulweather and Euchre Mountain quadrangles, Oregon: U. S. Geol. Survey open-file map.

SNAVELY, P. D., JR., RAU, W. W., HOOVER, LINN, JR., and ROBERTS, A. E.

1951. McIntosh Formation, Centralia-Chehalis coal district, Washington: Am. Assoc. Petroleum Geologists Bull., v. 35, n. 5, p. 1052-1061, 6 fig.

SNAVELY, P. D., JR., and VOKES, H. E.

1949. Geology of the coastal area between Cape Kiwanda and Cape Foulweather, oregon: U. S. Geol. Survey Oil and Gas Inv. Map OM 97. 
SNAVELY, P. D., JR, , and WAGNER, H, C.

1961. Differentiated gabbroic sills and associated

alkalic rocks in the central part of the Oregon Coast Range, Oregon: U. S. Geol. Survey Prof. Paper 424-D, P. D156-Dl61.

1963. Tertiary geologic history of western oregon and Washington: Wash. Div. Mines and Geology, Rept. Inves., n. 22,25 p., 23 fig.

1964. Geologic sketch of northwestern Oregon: U.S. Geol. Survey Bull. 1181-M, p. Ml-M17.

SNAVELY, P. D. , JR., WAGNER, H. C., and MACLEOD, N. S.

1969. Geology of western Oregon north of the Klamath Mountains, in Mineral and Water Resources of Oregon: Ore. Dept. Geol. and Min. Ind. Bull. 64, p. 32-46.

STEWART, R. E.

1956. Stratigraphic implications of some Cenozoic Foraminifera from western Oregon. OligoceneEocene: The ore Bin, v. 18, n. 7, p. 57-63.

1957. Stratigraphic implications of some Cenozoic Foraminifera from western oregon. Eocene: The ore Bin, v. 19, n. 2, p. 11-15.

SULLIVAN, F. R.

1962. Foraminifera from the type section of the San Lorenzo Formation, Santa Cruz County, California: Univ. Calif. Publ. Geol. Sci., v. 37, n. 4, p. 233-352, 23 pl., 5 fig.

THOMS, R. E.

1959. The geology and Eocene biostratigraphy of the southern Quimper Peninsula area, Washington: Unpub. M. S. thesis, Dept. Geol., Univ. Wash., 103 p., 7 pl., 20 fig.

1975. Biostratigraphy of the Umpqua Group, southwestern Oregon: Pacific Section, AAPG-SEPM Symposium, p. 513-562, 4 pl., 8 fig.

TIPTON, ANN, KLEINPELL, R. M., and WEAVER, D. W.

1973. Oligocene biostratigraphy, San Joaquin Valley, California: Univ. Calif. Publ. Geol. Sci., v. 105, 111 p., 14 pl., 12 fig.

TODD, M. R.

1953. Foraminifera from the lower Tertiary of Amchitka Island, Aleutian Islands: Cushman Found. Foram. Research, Contr., v. 4, pt. 1, p. 1-7, pl. 1-2. 
TODD, R:

1957a. Smaller Foraminifera, in Geology of Saipan, Mariana Islands. Part 3, Paleontology: U. S. Geol.

Survey Prof. Paper 280-H, p. 265-320, pl. 64-93.

1957b. Foraminifera from Carter Creek, northeastern

Alaska: U. S. Geol. Survey Prof. Paper 294-F, p. 223-234, pl. 28-29, 2 fig.

1966. Smaller For aminifera from Guam: U. S. Geol. Survey Prof. Paper 403-I, 41 p. , 19 pl., 2 fig.

1970. Smaller Foraminifera of late Eocene age from Eua, Tonga: U. S. Geol. Survey Prof. Paper 640-A,

23 p., 6 pl., 4 fig.

TODD, R., and LOW, D.

1967. Recent Foraminifera from the Gulf of Alaska and southeastern Alaska: U. S. Geol. Survey Prof. Paper 573-A, 46 p., 5 pl., 1 fig.

UJIIE, H. , and WATANABE, H.

1960. The Poronai Foraminifera of the northern Ishikari Coal-field, Hokkaido: Sci. Rept., Tokyo Univ. of Educ., sec. c, v. 7, n. 63, p. 117-147, 3 pl., 4 fig.

U. S. GEOLOGICAL SURVEY

1958. Suggestions to authors of the reports of the United States Geological Survey: 5th ed. , Washington, U. S. Government Printing office, 255 p.

WARREN, W. C., and NORBISRATH, HANS

1946. Stratigraphy of upper Nehalem River basin, northwestern Oregon: Am. Assoc. Petroleum Geologists Bull. v. 30 , n. 2, p. 213-237, 2 fig.

WARREN, W. C., NORBISRATH, HANS, and GRIVETTI, R. M.

1945. Geology of northwest Oregon west of the willamette River and north of latitude $45^{\circ} 15^{\prime}:$ U. S. Geol. Survey Oil and Gas Inv. Prelim. Map 42.

WATERS, J. N.

1968. Eocene faunule from the basal San Juan Bautista Formation of California: Cushman Found. Foram. Research, Contr., v. 14, pt. 1, p. 18-20, pl. 1-2.

WEAVER, D. W.

1962. Eocene Foraminifera from west of Refugio Pass, California: Univ. Calif. Publ. Geol. Sci., v. 37, n. 5, p. 353-420, 9 pl., 4 fig. 
WEAVER, D. W., and MOLANDER, G. E.

1964. The Eocene faunal sequence in the eastern Santa Rosa Hills, Santa Barbara County, California: Univ. Calif. Publ. Geol. Sci.. v. 4l, n. 3, p. 161-248, $18 \mathrm{pl}, 4$ fig.

WEAVER, W. R., and WEAVER, D. W.

1962. Upper Eocene Foraminifera from the southwestern Santa Ynez Mountains, California: Univ. Calif. Publ. Geol. Sci., v. 41, n. 1, 96 p., 22 pl., 4 fig.

WEINZIERL, L. L., and APPLIN, E. R.

1929. The Claiborne Formation on the coastal domes: Jour. Paleon., v. 3, n. 4, p. 384-410, pl. 42-44.

WILSON, E. J.

1954. Foraminifera from the Gaviota Formation east of Gaviota Creek, California: Univ. Calif. Publ. Geol. Sci.. v. 30, n. 2, p. 103-170, pl. 12-18, 3 fig. 

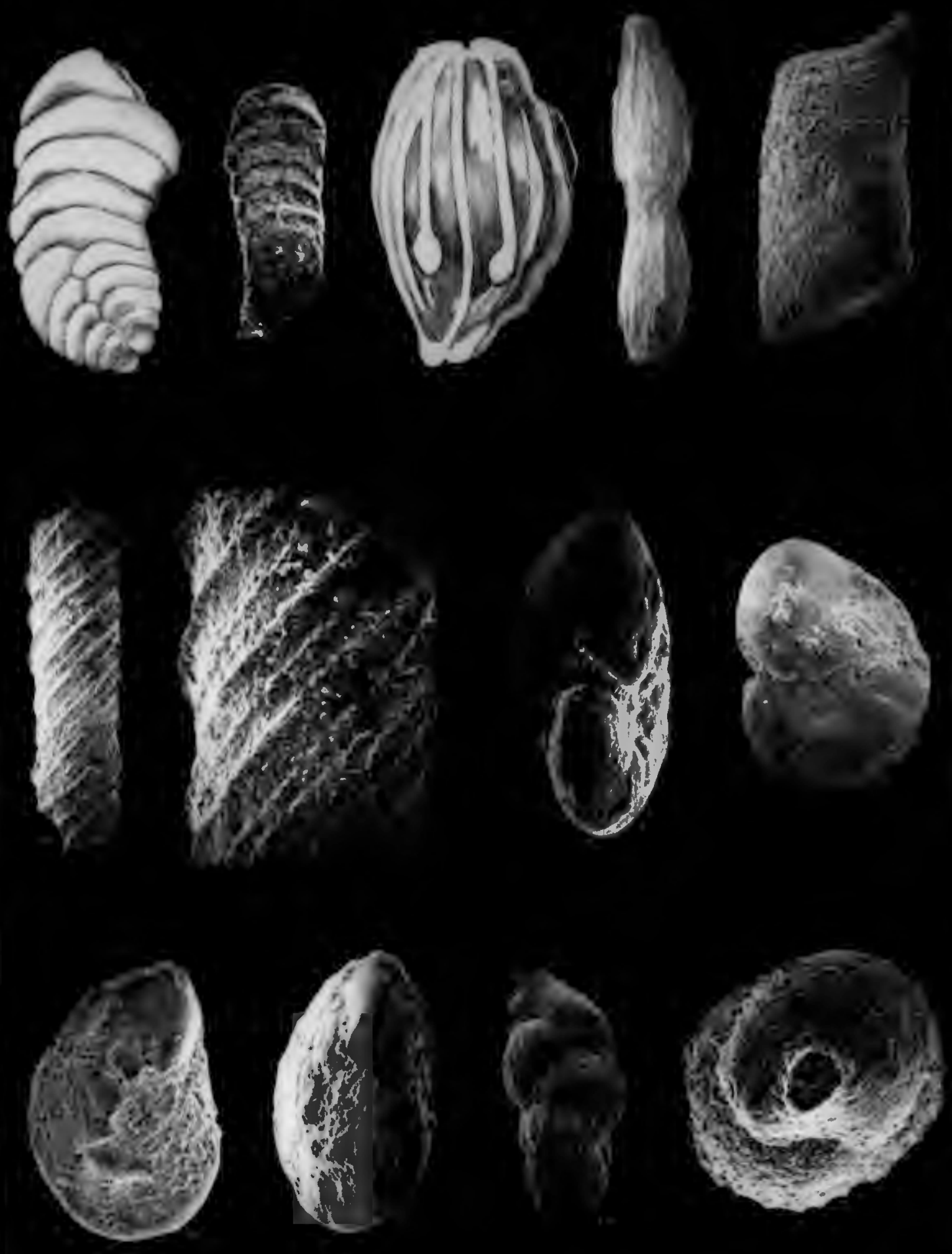